\title{
DIFFUSIOPHORESIS UNDER
}

\section{TURBULENT CONDITIONS}

\author{
by
}

PETER JOHN WHITMORE

B.Sc., Victoria University, Wellington, New Zealand, 1965

B.E., University of Canterbury, Christchurch, New Zealand, 1967

\author{
A THESIS SUBMITTED IN PARTIAL FULFILLMENT OF \\ THE REQUIREMENTS FOR THE DEGREE OF \\ DOCTOR OF PHILOSOPHY \\ in the Department \\ of \\ CHEMICAL ENGINEERING
}

We accept this thesis as conforming

to the required standard

THE UNIVERSITY OF BRITISH COLUMBIA

Ju1Y, 1976

(C) Peter John Whitmore, 1976 
In presenting this thesis in partial fulfilment of the requirements for an advanced degree at the University of British Columbia, I agree that the Library shall make it freely available for reference and study.

1 further agree that pemission for extensive copying of this thesis for scholarly purposes may be granted by the Head of my Department or by his representatives. It is understood that copying or publication of this thesis for financial gain shall not be allowed without my written pemission.

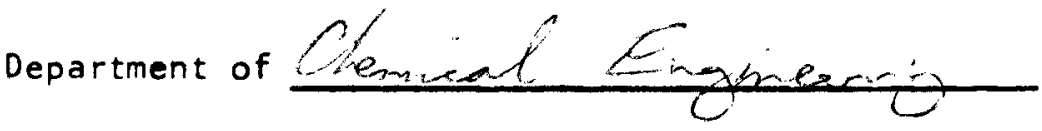

The University of British Columbia 2075 Wesbrook Place Vancouver, Canada V6T 1W5

Date $27-8-76$ 


\section{ABSTRACT}

This thesis describes both theoretical and experimental studies of diffusiophoresis, which is the movement of aerosol particles when they are suspended in a diffusing gas mixture. The phenomenon was studied under conditions of turbulent flow. Previous workers have demonstrated the efficacy of diffusiophoresis in continuously removing particles of the order of one micron $\left(10^{-6} \mathrm{~m}\right)$ in diameter from laminar gas streams. Such particles are difficult to remove by conventional gas cleaning techniques. However, any industrial application would probably require that the gas be in turbulent flow, to enable limitation of the equipment to an economic size. This observation provides the rationale for the current work, which can be divided into four major parts.

The first part is the derivation of an expression for the diffusiophoretic velocity of a large particle (i.e., a particle whose radius is large compared to the mean free path of the gas) in an undisturbed diffusing gas mixture. The continuum mechanics equations were solved in a more rigorous manner than was hitherto available. Previous derivations all implied the existence of diffusion slip at the particle surface, but this was shown to contravene the law of energy conservation. With 
zero slip, the particle was found to adopt the mean mass velocity of the fluid. This result, which was used in subsequent theory, differs from those of earlier workers.

The second part involves the development of a theory to predict the diffusiophoretic particle removal caused by diffusional mass transfer from a multicomponent gas stream. Two separate derivations were made, the more rigorous of which is based solely on the non-steady-state forms of the continuity equations for the particles and for the gas mixture. It is therefore independent of the patterns of mass transfer or gas flow. Theoretical predictions were derived for three possible values of the local particle velocity. These were the local mean mass velocity of the fluid, the local mean molar velocity, and a velocity suggested by Schmitt and Waldmann (1960).

The third part consists of experimental studies of particle removal by diffusiophoresis from turbulent gas streams. A binary gas mixture containing aerosol particles was passed up through a wetted wall column $(0.0254 \mathrm{~m} \mathrm{I.D.} \mathrm{and} 0.77 \mathrm{~m}$ in length), countercurrent to a flow of water. One component was insoluble, while the other was partially absorbed. The resulting particle removal was determined by measuring the inlet and outlet aerosol number concentrations in the gas. The soluble gases tested were ammonia and trimethylamine, while the insoluble gases were helium, methane, nitrogen, argon, and freon 12 (dichlorodifluoromethane). Uniform latex particles of $0.50,0.79,1.011,2.02$, and 5.7 microns in diameter were used. The Knudsen numbers for the particles ranged from 0.015 to 0.30 . 
The experimental results indicated that the theory relating particle removal to gas mass transfer was substantially correct. However, none of the theoretical predictions based on the three velocity expressions agreed with the experimental results for every case. It was concluded that this was because the particle diameters were sufficiently close to the mean free path of the gas that the particles fell into the transition regime, in which none of the velocity expressions apply precisely. The fourth part is a preliminary investigation into the practical application of diffusiophoresis for removing particulate matter from industrial gas streams. The importance of the type of equipment and the gases employed was demonstrated. It was concluded that diffusiophoresis used alone would not be economic except in special circumstances, but that it might be valuable when used in conjunction with other removal mechanisms. 


\section{TABLE OF CONTENTS}

\section{Page}

\section{ABSTRACT}

i

PREFACE

iv

LIST OF TABLES

$x i$

LIST OF FIGURES

xiii

ACKNOWLEDGEMENTS

xvii

Chapter

INTRODUCT ION

1

1.1 Preamble

1.2 Particle Removal from Industrial Gases

1.3 The Present Study

1.3.1 Objectives

1.3.2 Methods and scope

4

4

4

2

LITERATURE REVIEW

9

2.1 Early Work

2.2 Small Particles

2.3 Large Particles

2.4 Theories Encompassing the Transition Regime 
2.5 Continuum Equations

2.6 Experiments under Non-turbulent Conditions

2.7 Diffusiophoresis under Turbulent Conditions

2.8 Review Articles

3.1 Outline

3.2 The Continuum Mechanics Equations

3.3 Determination of the Free Particle velocity

3.4 The Rate of Energy Dissipation

3.5 Further Refinements and Additions

3.5.1 Non-Steady State Behaviour

3.5.2 A Second Order Approximation for Particle velocity

3.5.3 Particle Velocity in a Binary Gas Mixture with One Stagnant Component

3. 6 Conclusions

4.1 The Film Theory

4.2 The General Case

4.3 Discussion of Theory 
5.2 Apparatus 78

5.2.1 The Absorption System. 78

5.2.2 Wetted Wall Column Design 79

5.2.3 Gas and Liquid Supply systems 84

5.2.4 Aerosol Generation 86

5.2.5 Aerosol Particle Counter 86

5.2.6 Gas Analyser 91

5.3 Preliminary Procedure 92

5.3.1 Rotameter Calibration 92

5.3.2 Particle Content of Gases 93

5.3.3 Column Trials 93

5.3.4 Tests for Liquid Phase Mass
Transfer Resistance

5.4 Operation Procedure 94

5.4.1 Counter Calibration for Particle Size $\quad 94$

5.4.2 Counter Calibration for Gás Composition 95

5.4.3 Preparation for an Experimental Run 96

5.4.4 Sampling 98

$\begin{array}{ll}\text { 5.4.5 Particle Removal in the } & \\ & \text { Absence of Diffusiophoresis } \\ & 100\end{array}$

5.4.6 Isokinetic Sampling 100

5.5. Design of Experiments 101

6 RESULTS AND DISCUSSION 103

6.1 The Data 103

6.2 General Trends 105

6.3 Accuracy and Errors 107

6.3 .1 General 107 
6.3.2 Random Errors 108

6.3.3 Systematic Errors 108

6.4 Statistical Analysis 115

6.5 Detailed Analysis 122

6.5 .1 Helium-Ammonia 122

6.5.2 Methane-Ammonia 123

6.5.3 Nitrogen-Ammonia,
Preliminary Data

6.5.4 Nitrogen-Ammonia,
Short Column Data

6.5.5 Nitrogen-Ammonia 124

6.5.6 Argon-Ammonia 125

6.5.7 Freon 12-Ammonia 125

6.5.8 Nitrogen-Trimethylamine 126

$\begin{array}{lll}6.6 & \text { Further Discussion } & 127\end{array}$

7.1. General

7.2 Investigations by others 150

7.3 Optimal Vapour Use 150

7.4 Operating Cost Calculation 157

7.5 Reduction of Heat Requirements 158

7.6 Multiple Removal Mechanisms 161

7.7 Interactions between Mechanisms 162

8

$\begin{array}{ll}\text { SUMMARY AND CONCLUSIONS } & 166\end{array}$

8.1 Fundamental Theory 166

8.2 Derived Theory 167

8.3 Experimental Work 168

8.4 Practical Application 169 


\section{APPENDICES}

A

ROTAMETER CALIBRATION DATA

B

COLUMN MASS TRANSFER MODEL

B.1 Description

B. 2 Calculation and Results

C

DATA ANALYSIS AND SAMPLE CALCULATIONS

C.1 Source of Sample Data

C. 2 Reduction of Raw Data

C.3 Counter Calibration for Gas Composition

C. 4 Calculation Methods

C. 4.1 General

C.4.2 The Input Data

C.4.3 Calculation of Gas Mixture Properties

C.4.4 Calculation of the Particle Removal Efficiency

C.4.5 Calculation of the Theoretical Values for the Particlè Removal EEficiency

C.4.6 Calculation of Other Parameters

C.4.7 The Computed Results 
E.1 General Position of Data

E.2 Transition Regime Behaviour and Inertial Deposition 


\section{LIST OF TABLES}

$\underline{\text { Table }}$

$\underline{\text { Page }}$

I

Purchased Equipment

76

I I

Gases and Particles

77

III

Wetted Wall Column Design Parameters

81

IV

Estimate of Systematic Errors

113

$\mathrm{V}$

Comparison of Data with Theoretical Models

VI

Enhancement of Particle Capture

151

by Vapour Condensation

VII

Vapour Requirements to Attain 95\%

Particle Removal

VIII

Key to Calculation Program Nomenclature

196

IX

Listing of Calculation Program

199

$\mathrm{X}$

Physical Properties of Gases at 1 atm. and $20^{\circ} \mathrm{C}$

$\mathrm{XI}$

Data for $\mathrm{He}, \mathrm{NH}_{3}, 0.790$ Micron Diameter Particles

$\mathrm{XII}$

Data for $\mathrm{CH}_{4}, \mathrm{NH}_{3}, 0.790$ Micron

Diameter Particles

220

XIII

Data for $\mathrm{N}_{2}, \mathrm{NH}_{3}, 0.790$ Micron Diameter

Particles - Preliminary Runs

222 
Data for $\mathrm{N}_{2}, \mathrm{NH}_{3}, 0.790$ Micron

Diameter Particle. - Short Column

$\mathrm{XV}$

Data for $\mathrm{N}_{2}, \mathrm{NH}_{3}, 0.500$ Micron

Diameter Particles

XVI

Data for $\mathrm{N}_{2}, \mathrm{NH}_{3}, 0.790$ Micron

Diameter Particles

XVII

Data for $\mathrm{N}_{2}, \mathrm{NH}_{3}, 1.011$ Micron Diameter Particles

XVIII

Data for $\mathrm{N}_{2}, \mathrm{NH}_{3}, 2.020$ Micron

Diameter Particles - Series 1

241

XIX

Data for $\mathrm{N}_{2}, \mathrm{NH}_{3}, 2.020$ Micron

Diameter Particles - Series 2

$\mathrm{XX}$

Data for $\mathrm{Ar}, \mathrm{NH}_{3}, 0.790$ Micron

Diameter Particles

XXI

Data of $\mathrm{CF}_{2} \mathrm{Cl}_{2}$ (Freon 12), $\mathrm{NH}_{3}, 0.790$ Micron Diameter Particles

XXI I

Data for $\mathrm{CF}_{2} \mathrm{Cl}_{2}$ (Freon 12), $\mathrm{NH}_{3}, 1.011$

253

Micron Diameter Particles

XXIII

Data for $\mathrm{CF}_{2} \mathrm{Cl}_{2}$ (Freon 12), $\mathrm{NH}_{3}, 2.020$

Micron Diameter Particles

255

XXIV

Data for $\mathrm{N}_{2}, \mathrm{~N}\left(\mathrm{CH}_{3}\right)_{3}$ (Trimethylamine), 0.500 Micron Diameter Particles

$\mathrm{XXV}$

Data for $\mathrm{N}_{2}, \mathrm{~N}\left(\mathrm{CH}_{3}\right)_{3}$ (Trimethylamine), 0.790 Micrón Diameter Particles

XXVI

Data for $\mathrm{N}_{2}, \mathrm{~N}\left(\mathrm{CH}_{3}\right)_{3}$ (Trimethylamine), 1.011 Micron Diameter Particles

XXVII

Data for $\mathrm{N}_{2}, \mathrm{~N}\left(\mathrm{CH}_{3}\right)_{3}$ (Trimethylamine), 2.020 Micron Diameter Particles

XXVIII

Data for $\mathrm{N}_{2}, \mathrm{~N}\left(\mathrm{CH}_{3}\right)_{3}$. (Trimethylamine), 5.700 Micron Diameter Particles 


\section{LIST OF FIGURES}

Figure

Page

3.1 Coordinate systems.

5.1 General view of the equipment.

74

5.2

Closeup view of the wetted wall columns.

75

$5 \cdot 3$

Wetted wall column design - top.

82

5.4

Wetted wall column design - base.

5.5

Gas and liquid supply systems.

(Only one column shown.)

5.6 Electron micrograph of 0.50 micron

diameter latex particles.

Magnification $30,000 \mathrm{x}$.

5.7 Electron micrograph of 0.79 micron

diameter latex particles.

Magnification $10,000 \mathrm{X}$.

5.8

Electron micrograph of 1.011 micron

diameter latex particles.

Magnification $10,000 \mathrm{X}$.

5.9 Electron micrograph of 2.02 micron

diameter latex particles.

Magnification $10,000 \mathrm{x}$.

5.10 Electron micrograph of 5.7 micron

diameter latex particles.

Magnification 3,000 X. 
6.1

Results for helium, ammonia, 0.79 micron diameter particles. Inlet flow of transferred gas held constant at $6.0 \times 10^{-4} \mathrm{~m} 3 / \mathrm{sec}$.

6.2

Results for methane, ammonia, 0.79 micron diameter particles. Flow rate of inert gas held constant at $5.98 \times 10^{-4} \mathrm{~m}^{3} / \mathrm{sec}$.

$6 \cdot 3$

Results for nitrogen, ammonia, 0.79 micron diameter particles - short column. Flow rate of inert gas held constant at $6.0 \times 10^{-4} \mathrm{~m}^{3} / \mathrm{sec}$.

6.4

Results for nitrogen, ammonia, 0.50 micron diameter particles. Flow rate of inert gas held constant at 6. $0 . \times 10^{-4} \mathrm{~m}^{3} / \mathrm{sec}$.

6.5

Results for nitrogen, ammonia, 0.79 micron diameter particles. Flow rate of inert gas held constant at $6.0 \times 10^{-4}$ $\mathrm{m}^{3} / \mathrm{sec}$.

6.6

Results for nitrogen, ammonia, 0.79

micron diameter particles. Inlet flow rate of transferred gas held constant at $11.6 \times 10^{-4} \mathrm{~m} 3 / \mathrm{sec}$.

Results for nitrogen, ammonia, 0.79 micron diameter particles. Inlet gas composition held constant at 50 vo nitrogen.

6.8 Results for nitrogen, ammonia, 1.011 micron diameter particles. Flow rate of inert gas held constant at $6.0 \times 10^{-4}$ $\mathrm{m}^{3} / \mathrm{sec}$.

6.9 Results for nitrogen, ammonia, 2.02 micron diameter particles. Flow rate of inert gas held constant at $6.0 \times 10^{-4} \mathrm{~m}^{3} / \mathrm{sec}$. 
Results for argon, ammonia, 0.79 micron diameter particles. Flow rate of inert gas held constant at $5.6 \times 10^{-4} \mathrm{~m}^{3} / \mathrm{sec}$.

6.11

Results for freon $12\left(\mathrm{CF}_{2} \mathrm{Cl}_{2}\right)$, ammonia, 0.79 micron diameter particles. Flow rate of inert gas held constant at $0.98 \times 10^{-4} \mathrm{~m}^{3} / \mathrm{sec}$.

Results for freon $12\left(\mathrm{CF}_{2} \mathrm{Cl}_{2}\right)$, ammonia, 1.011 micron diameter particles. Flow rate of inert gas held constant at $0.98 \times 10^{-4} \mathrm{~m}^{3} / \mathrm{sec}$.

6.13

Results for freon $12\left(\mathrm{CF}_{2} \mathrm{Cl}_{2}\right)$, ammonia, 2.02 micron diameter particles. Flow rate of inert gas held constant at $0.98 \times 10^{-4} \mathrm{~m}^{3} / \mathrm{sec}$.

6.14

Results for nitrogen, trimethylamine $\left(\mathrm{N}\left(\mathrm{CH}_{3}\right)_{3}\right), 0.50$ micron diameter particles. Flow rate of inert gas held constant at $6.0 \times 10^{-4} \mathrm{~m}^{3} / \mathrm{sec}$.

6.15

Results for nitrogen, trimethylamine $\left(\mathrm{N}\left(\mathrm{CH}_{3}\right)_{3}\right), 0.79$ micron diameter particles. Flow rate of inert gas held constant at $6.0 \times 10^{-4} \mathrm{~m}^{3} / \mathrm{sec}$.

6.16 Results for nitrogen, trimethylamine $\left(\mathrm{N}\left(\mathrm{CH}_{3}\right)_{3}\right), 1.011$ micron diameter particles. Flow rate of inert gas held constant at $6.0 \times 10^{-4} \mathrm{~m}^{3} / \mathrm{sec}$.

6.17 Results for nitrogen, trimethylamine $\left(\mathrm{N}\left(\mathrm{CH}_{3}\right)_{3}\right), 2.02$ micron diameter

particles. Flow rate of inert gas held constant at $6.0 \times 10^{-4} \mathrm{~m}^{3} / \mathrm{sec}$.

Results for nitrogen, trimethylamine $\left(\mathrm{N}\left(\mathrm{CH}_{3}\right)_{3}\right), 5.7$ micron diameter particles. Flow rate of inert gas held constant at $6.0 \times 10^{-4} \mathrm{~m}^{3} / \mathrm{sec}$. 
7.1 Gas treatment in a stagewise process.

A. 1

Calibration curves for water and $32 \mathrm{w} \%$ caustic soda solution in the Brooks liquid rotameter. Liquid conditions$20^{\circ} \mathrm{C}$ and approximately 1 atmosphere.

A. 2

Calibration curves for nitrogen in the Brooks inert gas rotatmeter. Gas conditions - 4.40 atmospheres and $20^{\circ} \mathrm{C}$.

A. 3

Calibration curve for carbon dioxide in the size 3 Gilmont transferred gas rotameter. Gas conditions 1 atmosphere and $20^{\circ} \mathrm{C}$.

A. 4

Calibration curve for carbon dioxide in the size 4 Gilmont transferred gas rotameter. Gas conditions 1 atmosphere and $20^{\circ} \mathrm{C}$.

A. 5

Calibration curve for ammonia in the Brooks transferred gas rotameter. Gas conditions - 1 atmosphere and $20^{\circ} \mathrm{C}$.

B.1 Effect of liquid rate on outlet gas composition.

C. 1

Calibration correction ratio for nitrogen, ammonia, 0.7.9 micron diameter particles. The triangles denote mean values for a given mole fraction. 


\section{ACKNOWLEDGEMENTS}

I wish to thank my supervisor, Dr. Axel Meisen, who devoted many hours to our discussions, and made helpful and constructive suggestions throughout the course of this work.

Thanks are also due to other people in the Department of Chemical Engineering who have given me advice and assistance; in particular, the present and former staff of the workshop and stores, who helped me to construct the experimental equipment and maintain it in an operational state.

The manuscript was typed by Christine Fong, whose work is much appreciated.

I would like to thank the National Research Council, the University of British Columbia, and the Government of British Columbia for financial assistance.

Finally, at this point I would like to acknowledge the past help from my parents and teachers, and to thank my wife Jill for her support and for the confidence she has shown in me throughout this study. 


\section{PREFACE}

I have tried in this work to make a contribution to the general understanding of diffusiophoresis, rather than concentrating only on some specific problem. I hope that, by orienting this study in a practical direction, I have also produced some information of value in the control of particulate emissions in gas streams.

Peter Whitmore

Vancouver

July, 1976 


\section{INTRODUCTION}

\subsection{Preamble}

When an aerosol particle ${ }^{\dagger}$ is suspended in an isothermal diffusing gas mixture, it experiences a force which causes it to move. This phenomenon is termed diffusiophoresis. The force arises because the gas molecules bombard the particle with different masses and velocities. The analogous movement of a particle suspended in a gas through which heat is being conducted is called thermophoresis, and the two phenomena often occur together.

Diffusiophoresis plays a role in various natural processes such as aerosol capture during the formation and growth of rain droplets due to diffusion of water vapour. Another exàmple is the movement of dust particles in the lung during the transfer of oxygen, carbon dioxide, and water vapour.

The nature of the diffusiophoretic effect depends on

${ }^{\dagger}$ An aerosol is a solid or liquid disperse phase located in a continuous gas phase medium. The term aerosol particle is normally reserved for particles less than $1 \mathrm{~mm}$ in diameter. 
the size of the particle relative to the mean free path of the surrounding gas molecules. (Mean free paths of common gases are of the order of $0.05 \mathrm{\mu m}^{\dagger}$ under ambient conditions). Throughout this work particles will be described as "large" or"small" according to this criterion. Different laws have been found to govern the motion of particles of these two types, and those that fall into the intermediate transition regime.

\subsection{Particle Removal from Industrial Gases}

In many materials-handling and-processing operations the effluent gases contain suspended particles. The discharge of these particles to the atmosphere sometimes represents an economic loss to the process. Furthermore, it can have undesirable environmental effects, and hence may be regulated by law. It is therefore common practice to attempt to reduce the particulate loading of effluent gases before discharge.

In common gases and gas mixtures, at pressures of the order of 1 atmosphere and at typical process temperatures, particles greater than $500 \mathrm{\mu m}$ in diameter can usually be removed effectively by gravitational settling. For smaller particles methods based on inertial separation are often used. Standard cyclones can collect particles down to:about $10 \mathrm{\mu m}$, high efficiency cyclones to $5 \mu \mathrm{m}$, and various types of wet scrubbers as low as $0.5 \mu \mathrm{m}$.

$$
+1 \mu \mathrm{m}=10^{-6} \mathrm{~m}
$$


However, since inertial effects depend basically on the square of the particle diameter, gas velocities and hence power costs must rise rapidly as particle size decreases, in order to maintain a given removal efficiency. This places an economic limit on the use of these devices. Particles smaller than $0.1 \mu \mathrm{m}$ exhibit significant Brownian motion, and can therefore be removed by diffusional deposition in scrubbers or filters. These may also be used to remove large particles by inertial impaction and interception, but are only effective for particles of the order of $1 \mu \mathrm{m}$ at the expense of high gas pressure drop and power consumption.

Particles with diameters in the range of 0.1 to $5 \mu \mathrm{m}$ experience only weak diffusional and inertial effects, and are therefore often removed by electrostatic precipitation. However, the high cost makes this an expensive option. Also, particles with low electrical conductivities and gases that are not rich in electronegative molecules cause operating difficulties. There is consequently a need to examine unconventional methods for separating micron-sized particles from gases.

Diffusiophoresis is potentially attractive for this purpose because the induced particle velocity is largely independent of particle size. Since the mean free paths of most common gases are of the order of $0.05 \mu \mathrm{m}$ under ambient conditions, particles in the 0.1 to $5 \mu \mathrm{m}$ range should exhibit transition or large particle behaviour. Published experimental studies have demonstrated the efficacy of diffusiophoresis in removing particles from laminar gas streams, but practical application 
under these conditions is unlikely because of the low mass transfer rates of the vapour and: consequent large collecting surfaces required. However, under turbulent conditions, high mass transfer rates can be achieved, and high particle removal rates should be attainable.

\subsection{The Present study}

\section{3 .1 Objectives}

The following objectives were set for this work:

(i) The experimental study of diffusiophoresis under conditions of turbulent gas flow.

(ii) The development of a theory to explain the results obtained.

(iii) Testing of the existing fundamental theory of diffusiophoresis over a wider range of variables than previously used, and development of a new fundamental theory if required.

(iv) The study of diffusiophoresis in gas mixtures not previously used, and in gas mixtures whose components have widely different molecular weights.

\subsubsection{Methods and Scope}

Diffusiophoresis is associated with diffusion in gas mixtures due to concentration gradients. Such gradients can be established by bringing binary gas mixtures into contact with 
liquids or solids for which one gas component has a high affinity. In choosing an experimental system it was desirable that particles borne to the phase boundary not be re-entrained, and that the operation be essentially isothermal. It was also preferable that the absorbent or adsorbent be easy to handle, of low cost or else simple to regenerate, and that it offer low resistance to mass transfer. For these reasons solid adsorbents were ruled out, and all experiments were made using water as the absorption medium. This system was simple and allowed the experimental objectives to be met.

A wetted wall column was chosen to perform the absorption operation for the following main reasons:

(i) Simple construction.

(ii) Mass transfer area and liquid and gas velocities are well defined compared to most other types of mass transfer equipment.

(iii) If the column is constructed of glass, the behaviour in the interior can be observed.

(iv) Particle capture due to inertial mechanisms should be lower than in equipment such as packed towers where sudden changes in gas flow direction occur.

The last reason is important in attempting to study diffusiophoresis in isolation from other removal mechanisms.

In the experiment, particles were added to an inert (non-absorbable) gas stream using an aerosol generator. This stream was then mixed with a stream of the gas to be transferred, 
to give the required feed composition. As the resulting mixture flowed up through the wetted wall column, part of the gas was absorbed and a fraction of the particles were collected in the downflowing water. The inlet and outlet gas streams were analysed for gas composition by orsat analysis, and for particle concentration using a commercially available optical counter. The fractional particle removal obtained from these measurements could then be related to the change in gas composition in the equipment.

\begin{tabular}{|c|c|}
\hline Inert species & Transferred species \\
\hline helium & ammonia \\
\hline methane & ammonia \\
\hline nitrogen & ammonia \\
\hline argon & ammonia \\
\hline $\begin{array}{l}\text { freon } 12 \\
\text { (dichlorodifluoromethane) }\end{array}$ & ammonia \\
\hline nitrogen & trimethylamine \\
\hline
\end{tabular}

Particles of diameter $0.5,0.79,1.011,2.02$, and $5.7 \mu \mathrm{m}$ were used.

Once the fundamental theory giving the velocity of a particle in an undisturbed diffusing gas mixture is known, a "derived" theory relating particle removal to mass transfer rate in the wetted wall column can be developed. Under laminar gas flow conditions this problem is conceptually relatively simple, since the continuum mechanics equations completely define the 
system and allow the particle velocity to be calculated at any point. However, in practice it is exceedingly difficult since non-linear partial differential equations must be solved.

For turbulent flows another approach must be used, since local conditions in the turbulent core are unknown. In the original derivation made during this study, particle transfer was treated in an analogous manner to mass transfer from a turbulent fluid through a laminar film. This approach was very satisfactory since it allowed several variables to be eliminated and very simple results to be obtained.

The original theory was not completely rigorous in that it assumed the mass transfer boundary layer to be laminar, when in fact it is partly turbulent. Subsequently, however, a more powerful theory, which did not require this assumption, was derived by combining the gas and particle continuity equations. The new theory confirmed the results of the original one, and showed them to be true regardless of conditions in the boundary layer or even in the core. Thus the improved theory holds even for laminar flow.

All published fundamental theories for the diffusiophoretic velocity of a large particle in an undisturbed diffusing gas mixture either invoke the concept of diffusion slip at the particle surface directly, or imply its existence by their results. Diffusion slip refers to the non-zero velocity of a gas in contact with a solid surface, which is claimed to arise in diffusing systems. It was difficult to understand intuitively how this 
slip could be present around a particle, and eventually it was found that its existence violated energy conservation in the system. This revelation led to the development of a new theory of diffusiophoresis for large particles.

Diffusiophoresis could be employed in a practical gas cleaning system either entirely on its own, or else to enhance and/or supplement particle removal by other mechanisms. A preliminary economic evaluation of the former possibility requires not only information on particle collection rates, but also knowledge of the optimum method of using the transferred vapour. This involves questions such as what vapour concentrations to use, whether to adopt a stagewise operation, and whether to attempt heat recovery for further vapour generation. The other possibility, of employing diffusiophoresis together with other removal mechanisms, cannot be assessed at this time without more specific details, and a better understanding of how diffusiophoresis operates in conjunction with these mechanisms. 
Chapter 2

\section{LITERATURE REVIEW}

\subsection{Early Work}

The first known report of the existence of diffusiophoresis is contained in the voluminous paper by Aitken (1883). He observed the formation of dust-free spaces next to moist surfaces, and correctly attributed them to the evaporation of water. However, he made no attempt to find a quantitative relationship between the magnitude of the dust-free space and evaporation rate or particle size.

Derjaguin and Dukhin (1956a, 1956b, 1957) predicted the existence of the effect theoretically. They were apparently unaware of Aitken's earlier work. Their interest in the forces between two evaporating droplets seems to have led them to the realisation that diffusing gas mixtures can cause particle motion. Initially they postulated that the particle adopts the Stefan velocity, that is the gas mean mass velocity which stefan (1881) had shown to arise in diffusing mixtures. Later they recognized that the particle behaviour is influenced by the Knudsen number, $\mathrm{Kn}$, which is the ratio of gas mean free path to 
particle radius. The value of this number can be used to delineate the continuum $(K n \ll 1)$, transition $(K n \simeq 1)$, and free molecule $(\mathrm{Kn}>>1)$ regimes ${ }^{\dagger}$; and the particles failing into these may be described as large, intermediate, or small in comparison to the mean free path of the gas.

The first modern experimental proof of the existence of diffusiophoresis was provided by Freise (1957) and Facy $(1958 \mathrm{a} ; \mathrm{b})$. These authors also appear to have been unaware of Aitken's work. Freise demonstrated the movement of small particles in diffusing liquids, and estimated that they moved at approximately the Stefan velocity. Facy made some qualitative studies on the movement of small particles in air near growing and evaporating droplets and also attempted to develop theoretical expressions for particle velocity. He recognized the importance of Knudsen number, and by following the technique Einstein (1924) had used to derive an approximate theory for thermophoresis, he was able to deduce the general forms of the velocity expressions for small and large particles.

\subsection{Small Particles}

The first comprehensive theory of diffusiophoresis was

'In the continuum regime the gas properties can be regarded as continuous, whereas in the free molecule regime the molecular behaviour becomes important. 
developed by Derjaguin and Bakanov (1957) for a small spherical particle. They used a molecular mechanics approach, and recognized that the velocity distribution of molecules striking the particle closely resembles that in the bulk of the gas. The distribution of the incident molecules can therefore be obtained from the Chapman-Enskog formulae, as given by Chapman and Cowling (1964). By assuming specular (i.e. elastic) molecule-particle collisions, Derjaguin and Bakanov were also able to find the velocity distribution of the rebounding molecules. The diffusiophoretic velocity was then calculated by setting the net momentum transfer to the particle equal to zero.

Waldmann (1959) independently conceived a similar theory, but assumed (more realistically) that the moleculeparticle collisions were partly specular and partly diffuse. His expression for the diffusiophoretic velocity of a small particle located in a binary gas mixture undergoing equimolar counter-diffusion is ${ }^{\dagger}$

$$
\underline{v}_{p}=-\frac{\left(1+\frac{\pi}{8} \beta_{2}\right) \sqrt{m_{2}}-\left(1+\frac{\pi}{8} \beta_{1}\right) \sqrt{m_{1}}}{\left(1+\frac{\pi}{8} \beta_{1}\right) \gamma_{1} \sqrt{m_{1}}+\left(1+\frac{\pi}{8} \beta_{2}\right) \gamma_{2} \sqrt{m_{2}}} D\left(\underline{\nabla} \gamma_{2}\right)_{\infty} .
$$

'It is usual in the literature to express velocities in terms of the concentration gradient of component 1 . The subscripts " 1 " and " 2 " in the remainder of the expression would then appear in the reverse order to that given here. However, the convention used in this work is more convenient when dealing with multicomponent mixtures. 
Here $m_{i}, \beta_{i}$ and $\gamma_{i}$ are the molecule mass, accomodation coefficient (fraction of molecules reflected diffusely from the surface), and mole fraction of species $i$ respectively, $D$ is the gas diffusivity, and $\left(\underline{\nabla}_{\gamma_{2}}\right)_{\infty}$ is the concentration gradient in the undisturbed gas. The velocity $\underline{v}_{p}$ is measured here with respect to the gas mean molar velocity. Later, Schmitt (1961) showed how the theory could be modified for the case where component 2 of the gas diffuses through component 1 which is stagnant. He found the particle velocity measured with respect to the stagnant component to be

$$
\underline{v}_{\mathrm{p}}=-\frac{\left(1+\frac{\pi}{8} \beta_{2}\right) \sqrt{\mathrm{m}_{2}}}{\left(1+\frac{\pi}{8} \beta_{1}\right) \gamma_{1} \sqrt{\mathrm{m}_{1}}+\left(1+\frac{\pi}{8} \beta_{2}\right) \gamma_{2} \sqrt{\sqrt{\mathrm{m}_{2}}}} \frac{\mathrm{D}}{\gamma_{1}}\left(\underline{\nabla} \gamma_{2}\right)_{\infty}:
$$

Subsequent improvements in the diffusiophoretic theory for small particles have been minor. Bakanov and Derjaguin (1960) published another theoretical paper similar to their earlier one, but allowed for a temperature gradient in the gas. They improved the accuracy of the analysis by carrying an extra term in the velocity distribution function for the molecules. When rewritten in term of common gas properties, and if temperature effects are ignored, their expression for equimolar counter-diffusion becomes

$$
\underline{v}_{p}=-\left(\frac{\sqrt{m_{2}}-\sqrt{m_{1}}}{\gamma_{1} \sqrt{m_{1}}+\gamma_{2} \sqrt{m_{2}}}-\frac{1}{2} \frac{1}{d_{0} \gamma_{1} \gamma_{2}} \frac{\gamma_{1} d_{1}+\gamma_{2} d_{-1}}{\gamma_{1} \sqrt{m_{1}}+\gamma_{2} \sqrt{m_{2}}}\right) D\left(\underline{\nabla} \gamma_{2}\right)_{\infty},
$$

where $d_{0}, d_{1}$ and $d_{-1}$ are parameters dependent on the gas properties. 
The first term agrees with Waldmann's expression when the accommodation coefficients are unity. The second term arises from the use of a better approximation to the distribution function, and is of the same order as $-\alpha_{\mathrm{T}} / 5$ (and equal to $-\alpha_{\mathrm{T}} / 5$ when $\mathrm{m}_{1} \sim \mathrm{m}_{2}$ and $\gamma_{1} \ll \gamma_{2}$ or $\left.\gamma_{2}:<\gamma_{1}\right)$. However, the thermal diffusion factor ${ }^{\dagger}, \alpha_{\mathrm{T}^{\prime}}$ is normally small, so that this term can usually be neglected.

Derjaguin and Yalamov (1972) later presented the theory in greater detail. The essential features of their expression can be easily seen in the case where the accommodation coefficients are unity (which is close to reality).

$$
\begin{aligned}
& \underline{v}_{p}=-\int \frac{\sqrt{m_{2}}-\sqrt{m_{1}}}{\gamma_{1} \sqrt{m_{1}}+r_{2} \sqrt{m_{2}}} \\
& \left.-\frac{1}{1+\frac{\pi}{8}} \frac{1}{d_{0} \gamma_{1} \gamma_{2}} \frac{\gamma_{1} d_{1}+\gamma_{2} d_{1}}{\gamma_{1} \sqrt{m_{1}}+\gamma_{2} \sqrt{m_{2}}}\right) D\left(\nabla \gamma_{2}\right)_{\infty}
\end{aligned}
$$

In comparison with Bakanov and Derjaguin's (1960) result, the

†Thermal diffusion is the tendency of a gas mixture to separate when subjected to a temperature gradient; and the thermal diffusion factor is a constant related to the rate of separation. 
second term is now multiplied by $1 /\left(1+\frac{\pi}{8}\right)$, i.e. $1 / 1.393$, in place of the previous factor of $1 / 2$.

$$
\text { Mason and Chapman (1962) rederived the theory by }
$$

regarding the suspended particle as a large molecule. They assumed a combination of specular reflection, and diffuse reflection but at unchanged speed. The final result is identical to Waldmann's except that the $\frac{\pi}{8}$ factor multiplying the accomodation coefficients is replaced by $4 / 9$.

\subsection{Large Particle}

The derivation of a satisfactory theory for large particles has proven much more difficult. For this molecular regime one might think that the continuum mechanics equations would describe the system adequately, and yield a solution for the particle velocity. However, it has generally been believed that these equations do not hold close to the particle because of the complex interactions of molecules with the particle surface and with each other. In this case the methods of molecular mechanics must be used here. Nor is the solution of the continuum mechanics equations themselves straightforward, since fluid properties are not constant in diffusing systems, and time-dependent terms are present.

The work of Kramers and Kistemaker (1943) has had an important influence on the subsequent development of theory for large particles. These authors had predicted theoretically that 
when a binary gas mixture diffuses parallel to a flat plane of infinite extent, the velocity of the gas is not generally zero at the surface (or more strictly of the order of one mean free path away from the surface). This velocity discontinuity they termed diffusion slip. Kramers and Kistemaker's derivation of the slip velocity is based on a momentum balance in the direction of diffusion, for molecules arriving at and leaving the surface. The net momentum transfer to the surface is then set to zero to yield

$$
v_{\text {slip }}=-\left(\frac{m_{2}-m_{1}}{\gamma_{1} m_{1}+r_{2} m_{2}}-\frac{\sqrt{m_{2}}-\sqrt{m_{1}}}{\gamma_{1} \sqrt{m_{1}}+\gamma_{2} \sqrt{m_{2}}}\right) D \frac{d \gamma_{2}}{d x}
$$

where $d r_{2} / d x$ is the mole fraction gradient along the surface. Two important simplifications were made in their treatment. Maxwelliam methods were assumed adequate to yield an approximate momentum balance, and interactions between incoming and rebounding molecules near the surface were ignored. Later derivations of slip velocity are similar, but attempt to improve on these simplifications. An important implicit condition, which is not relaxed in later treatments, is that the shear stress on the surface is zero.

Kramers and Kistemaker also attempted to detect slip experimentally. In a well-designed experiment, air and hydrogen were allowed to diffuse in opposite directions through a capillary tube, and the resulting pressure drop across the tube was measured. 
Using elementary methods of fluid dynamics they predicted that the existence of slip would approximately halve the pressure drop along the tube compared to that without slip. Their measurements indicated that the reduction in pressure drop was of this order, and they therefore concluded that slip existed.

The first complete derivation of an expression for the diffusiophoretic velocity of a large particle was presented by Schmitt and Waldmann (1960). They solved the continuum mechanics equations subject to a boundary condition at the particle surface that allows for the non-continuum behaviour in this region. The following simplified forms of the continuum mechanics equations were used in order to find the velocity of a spherical particle in a binary gas mixture undergoing equimolar counter-diffusion:

$\begin{array}{lrl}\text { Navier-Stokes (NS) } & \mu \underline{\nabla^{2}} \underline{v}=\underline{\nabla p} \\ \text { Total continuity (TC) } & \underline{\nabla} \cdot \underline{v}=0 \\ \text { Partial continuity (PC) } & \underline{\nabla}^{2} \gamma_{2}=0\end{array}$

These equations have also been used in most later theories, and a discussion of them is deferred till later in this chapter. The boundary condition at the particle surface was obtained by replacing the gradient in Kramers and Kistemaker's slip expression $d \gamma_{2} / d x$, by the equivalent form for a sphere, $\left(1 / r d \gamma_{2} / d \theta\right)_{s} \cdot$ The validity of this step is in doubt, because, while the shear stress might vanish for equimolar counter diffusion over a flat surface, it does not vanish for a curved surface. The same transition was also made by later workers. 
From the solution of the partial continuity equation, Schmitt and Waldmann were able to evaluate the concentration gradient along the particle surface, $\left(d \gamma_{2} / d \theta\right)_{s}$, and thus find the slip condition and determine the particle velocity.

$$
\underline{v}_{p}=-\frac{\sqrt{m_{2}}-\sqrt{m_{1}}}{r_{1} \sqrt{m_{1}}+\gamma_{2} \sqrt{m_{2}}} D\left(\underline{\nabla}_{2}\right)_{\infty}
$$

This expression indicates that the particle velocity is in the same direction, but is always smaller than the mean mass velocity of the gas mixture,

$$
\underline{w}=-\frac{m_{2}-\underline{m}_{1}}{\gamma_{1} m_{1}+\gamma_{2} m_{2}} \quad D\left(\underline{\nabla} \gamma_{2}\right)_{\infty}
$$

The mean molar velocity is of course by definition zero. The fact that the particle velocity does not depend on particle size had been deduced earlier by Facy (1958a).

$$
\text { Brock (1963) refined the theory of slip at a flat }
$$

surface. He used the Chapman-Enskog equations (see Chapman and Cowling (1964)) to allow for the non-Maxwellian nature of the gas. The effect of interactions between incident and rebounding molecules near the surface was assumed to be negligible. Hence these equations could be used to calculate the incoming molecular momentum. To describe the behaviour of molecules leaving the surface he introduced Maxwell's empirical accommodation coefficients. 
A fraction of $\beta_{i}$ of the molecules of species $i$ reaching the surface are assumed to rebound diffusely, and the remainder to undergo specular. collisions.

Brock's slip expression is of limited utility since it contains accommodation coefficients which can only be determined experimentally. However, according to Derjaguin and Yalamov these coefficients can be approximated as unity, in which case the expression becomes for binary gas mixtures

$$
\begin{aligned}
& v_{\text {slip }}=-\left(\frac{m_{2}-m_{1}}{r_{1} m_{1}+r_{2} m_{2}}-\frac{\sqrt{m_{2}}-\sqrt{m_{1}}}{r_{1} \sqrt{m_{1}}+r_{2} \sqrt{m_{2}}}\right. \\
& -\frac{1}{2} \frac{1}{d_{0} \gamma_{1} \gamma_{2}} \frac{\gamma_{1} d_{1}+\gamma_{2} d_{1}}{\gamma_{1} \sqrt{m_{1}}+\gamma_{2} \sqrt{m_{2}}} \quad \quad D \frac{d \gamma_{2}}{d x}
\end{aligned}
$$

Brock discusses the force on a particle in a diffusing system for various cases. However, it is more illustrative to give a Schmitt and Waldmann-type solution for particle velocity, using Brock's slip expression as the boundary condition:

$$
\begin{aligned}
\underline{v}_{\mathrm{p}}= & -\left(\frac{\sqrt{\mathrm{m}_{2}}-\sqrt{\mathrm{m}_{1}}}{\gamma_{1} \sqrt{\mathrm{m}_{1}}+\gamma_{2} \sqrt{\mathrm{m}_{2}}}\right. \\
& \left.-\frac{1}{2} \frac{1}{\mathrm{~d}_{0} \gamma_{1} \gamma_{2}} \frac{\gamma_{1} \mathrm{~d}_{1}+\gamma_{2} \mathrm{~d}_{1}}{\gamma_{1} \sqrt{\mathrm{m}_{1}}+\gamma_{2} \sqrt{\mathrm{m}_{2}}}\right) D\left(\underline{\nabla} \gamma_{2}\right)_{\infty} .
\end{aligned}
$$


Brock's analysis is valid for the dilute case $\left(\gamma_{2} \ll \gamma_{1}\right)$, and for small concentration gradients. When $\mathrm{m}_{1} \sim \mathrm{m}_{2}$ ' the first term corresponds to that of Schmitt and Waldmann's expression, and the second term can be simplified using the thermal diffusion ratio to give

$$
-\frac{1}{5} \quad \alpha_{\mathrm{T}} \quad \mathrm{D}\left(\underline{\nabla} \gamma_{2}\right)_{\infty}
$$

Thus the contribution of the second term is minor, but it does indicate that for $\mathrm{m}_{1} \simeq \mathrm{m}_{2}$, the particle may travel in either direction, depending on the sign of $\alpha_{T}$. It is no coincidence that Brock's expression for large particles is identical to that derived by Bakanov and Derjaguin (1960) for small particles (when the accommodation coefficients are unity). This is a consequence of the assumption, made in both cases, that moleculemolecule interaction near the surface is negligible.

Derjaguin and Yalamov (1972) attempted to improve Brock's analysis by allowing for molecular interactions near the particle surface. Even though the work was restricted to dilute systems $\left(\gamma_{2} \ll \gamma_{1}\right)$ with small concentration gradients, the calculations become very involved, and the results are expressed in terms of collision integrals which must be calculated numerically. The predicted slip velocity is

$$
v_{\text {slip }}=\frac{\delta \sqrt{\frac{m_{2}}{m_{1}}}-\sqrt{\frac{m_{2}}{m_{1}}}}{r_{1}} D \frac{d \dot{r}_{2}}{d x}
$$


where $\delta$ is a function of several collision integrals. Comparison with Kramers and Kistemaker's slip expression for the case where $\gamma_{2} \ll \gamma_{1}$ and $m_{1} \sim m_{2}$ shows that Derjaguin and Yalamov's derivation results in an extra term

$$
(\delta-1) \quad \sqrt{\frac{m_{1}}{m_{2}}} \frac{D}{r_{1}} \frac{d \gamma_{2}}{d x}
$$

For the air-water vapour system $\delta$ is. given as 1.253 so that

$$
v_{\text {slip }}=0.365 \frac{\mathrm{D}}{\gamma_{1}} \frac{\mathrm{d} \gamma_{2}}{\mathrm{dx}}
$$

Kramers and Kistemaker's proportionality constant evaluated for the same system is 0.167 . Intuition strongly suggests that any interaction between incoming and rebounding molecules should reduce the slip velocity, not increase it. This prediction must therefore be viewed with some scepticism.

An entirely different approach to diffusiophoresis was taken by Derjaguin et al. (1966) who used a "phenomenological" technique. This method relies on a combination of irreversible thermodynamics and continuum mechanics to determine particle velocity. They consider two reservoirs containing different gases and separated by a porous "partition" made up of randomly arranged spheres rigidly fixed in space, at a spacing much larger than their radius. The behaviour of this system is used to deduce 
the velocity of a free aerosol particle in the gas mixture. Slip was assumed to be insignificant.

In their derivation the authors make a correction for local diffusion streams caused by pressure gradients around the particle. However, since pressure diffusion can be shown to be insignificant compared to ordinary diffusion in this problem (see for example Bird et al.(1962)), and since their diffusion equation is not directly coupled to their momentum equation, it is unclear why this correction is necessary. The final expression obtained for the particle velocity is equivalent to the gas mean molar velocity in their system, although the authors do not state this explicitly. Using their assumption of negligible slip, the particle velocity can be found directly from the continuum equations given in their paper, without recourse to thermodynamic methods. This yields a different result.

Derjaguin and Yalamov (1972) later presented the approach in more detail, allowing for the existence of slip at the particle surface. They concluded that normal diffusion slip and baro-: or pressure-diffusion slip exactly cancelled out. Thus the problem became equivalent to their previous one and yielded the same result.

Derjaguin and Yalamov (1972) compared their predicted particle velocities derived from the "phenomenological" method with the results obtained by solving the continuum mechanics equation using their slip boundary condition. The two methods showed reasonable agreement for the air-water vapour system and they concluded that the difference was due to numerical errors 
arising in the second method. If this were the case, then one could deduce that the slip velocity past a flat surface would always be such that the mean molar velocity is zero with respect to the surface. Despite the agreement, there are sufficient areas of concern to cast doubt on the accuracy of both their theories.

\subsection{Theories Encompassing the Transition Regime}

Analysis of particle behaviour in the transition

regime ( $\mathrm{Kn} \sim 1)$ presents major difficulties, and was first attempted by Brock (1968). He adopted a first-order perturbation technique to calculate the velocity distribution function for the gas molecules near the particle. This gave the particle velocity

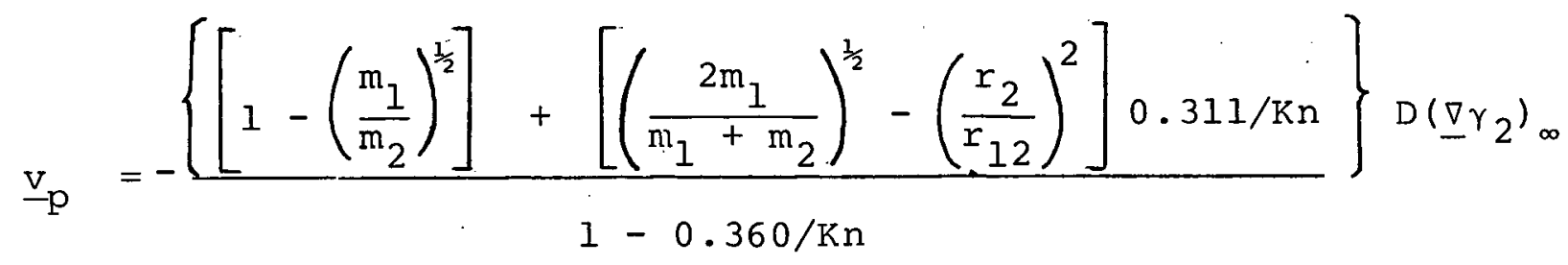

where $r_{12}=\left(r_{1}+r_{2}\right) / 2$ and $r_{1}$ and $r_{2}$ are the effective molecular radii of species 1 and 2 . Since the derivation requires that $\gamma_{2} \gg \gamma_{1}$, and hence $\gamma_{1} \sqrt{m_{1}}+\gamma_{2} \sqrt{m_{2}} \simeq m_{2}^{\frac{1}{2}}$, the equation reduces to the simple velocity expression for small particles when the Knudsen number is large. For small Knudsen numbers it gives 


$$
\underline{v}_{p}=-\left\{\left(\frac{r_{2}}{r_{12}}\right)^{2}-\left(\frac{2 m_{1}}{m_{1}+m_{2}}\right)^{\frac{1}{2}}\right\} 0.864 D\left(\underline{\nabla} \gamma_{2}\right)_{\infty}
$$

The lack of agreement between this and other large particle theories is indicative of the approximate nature of the derivation. Brock found moderate agreement between his theory and selected experimental results of Schmitt and Waldmann (1960). Annis et al. (1973) used a novel technique which they claim allows the derivation of a theory that holds for all particle sizes. The groundwork for their method is laid in an earlier paper (1972) dealing with the drag on a particle in a non-diffusing gas. In their method the particle is treated as a large molecule, and the molecular diffusion equation (see Hirschfelder et al. (1964)) is used to obtain the particle velocity.

Their general velocity expression is a function of two variables, both closely related to the knudsen number. For small particles and equimolar counter-diffusion it yields

$$
\underline{v}_{p}=-\frac{\left(c_{2}^{*} / a_{2}^{*} b_{2}^{*}\right) \sqrt{m_{2}}-\left(c_{1}^{*} / a_{1}^{*} b_{1}^{*}\right) \sqrt{m_{1}}}{\left(c_{1}^{*} / a_{1}^{*} b_{1}^{*}\right) \gamma_{1} \sqrt{m_{1}}+\left(c_{2}^{*} / a_{2}^{*} b_{2}^{*}\right) \gamma_{2} \sqrt{m_{2}}} D\left(\underline{\nabla} \gamma_{2}\right)_{\infty},
$$

where $a_{i}^{*}, b_{i}^{*}$, and $c_{i}^{*}$ depend on the interaction of molecules with the particle surface. This is of the same form as Waldmann's (1959) expression. 


$$
\underline{v}_{p}=-\frac{\left(1 / a_{2}^{*}\right) \sqrt{m_{2}}-\left(1 / a_{1}^{*}\right) \sqrt{m_{1}}}{\left(1 / a_{1}^{*}\right) \gamma_{1} \sqrt{m_{1}}+\left(1 / a_{2}^{*}\right) \gamma_{2} \sqrt{m_{2}}} D\left(\nabla \gamma_{2}\right)_{\infty}
$$

which agrees with Schmitt and Waldmann's (1960) expression when the interactions of both molecular species with the sphere are similar, i.e., $a_{1}^{*}=a_{2}^{*}$. In a later paper Annis and Mason (1975) expanded the theory to include also thermophoresis, and showed that when both temperature and concentration gradients are present, diffusiophoresis is augmented by a thermal diffusion contribution as well as a small additional coupling term.

Although this method is excellent for small particles, its application in the transition and continuum regimes is unsatisfactory. The diffusion equation, on which the theory rests, is correct only when the particle radius is much smaller than the gas mean free path. Large particles do not alter the surrounding gas concentration, as this equation implies, but rather form a separate phase in the system.

\subsection{The Continuum Equations}

We return now to an examination of the continuum mechanics equations. The simple forms given earlier were used by all authors except Brock (1963). Although intuition might suggest the use of these forms, no one has rigorously established their validity in this problem. Schmitt and Waldmann (1960) used the equations essentially without comment. Derjaguin et al. 
(1966) assumed that the simple equations were valid for low velocities and a small relative density of one component. This was indicated in two earlier papers by Derjaguin and Dukhin (1956). A partial justification for the validity of the equations under the same conditions was later given by Derjaguin and Yalamov (1972). Brock used a more accurate form of the partial continuity equation and showed how this could be simplified in certain cases. Systematic simplification of the three basic equations is therefore still lacking.

\subsection{Experiments under Non-turbulent Conditions}

The primary objective of most studies has been to measure particle velocity for comparison with the various theories. Some workers have also been interested in demonstrating the ability of diffusiophoresis to remove particles on a continuous basis from a gas stream.

The earliest quantitative velocity measurements are those of Schmitt and Waldmann (1960). These were made in a modified Millikan oil drop apparatus (see Millikan (1911)) consisting of a vertical glass tube connected at each end to a flask containing a pure gas. Thus a binary diffusion field could be established in the tube. Two charged plates were used to hold silicon oil droplets in a fixed position when required, and the droplet velocities were directly measured by microscope.

Schmitt and Waldmann's work has been severely criticised by Derjaguin and Yalamov (1972) because of the high experimental error and the possibility of extraneous convective gas flows in 
the apparatus. In addition, although schmitt and Waldmann obviously believed that diffusion slip existed, they gave no indication that they made an allowance for this effect at the tube walls. Furthermore, they do not mention the importance of aerosol position with respect to the tube wall, and it is therefore possible that they did not properly allow for the parabolic gas velocity profile. In any case their data do not agree with any proposed theory. Their work must therefore be discounted. The same criticisms apply to the later work of Schmitt (1961) for steam-air mixtures.

Schmitt's paper also mentions a simple particle collector made of two horizontal parallel plates between which air containing cigarette smoke was passed. Steam was injected through the upper porous asbestos plate and condensed on the lower copper plate. No operational details are given except that the collector was able to completely remove the smoke from the air.

Goldsmith, Delafield and Cox (1963a, 1963b) also worked with a parallel plate collector, but in their design one plate was lined with absorbent paper and saturated with water, while the other was lined with paper saturated with sulfuric acid. Very small radioactive particles were passed in an air stream between the plates, and the deposited particles were detected by their radiation. From the measurements Goldsmith et al. were able to deduce the particle velocity, which agreed well with Waldmann's (1959) theoretical expression for small particles when the accommodation coefficiencies in this expression were unity. 
Storozhilova (1964) devised a different technique for measuring particle velocity, whereby a fine stream of aerosol particles was injected into a fully developed laminar flow between two parallel plates. A concentration gradient was maintained between the plates, and the diffusiophoretic velocity could be deduced from the trajectory of the particles as observed by microscope. All experiments were made using the air-water vapour system. Although this type of experiment may eliminate some sources of error, the data showed considerable scatter, particularly for Knudsen numbers of less than 1. Furthermore, the experiments extended only down to knudsen numbers of about 0.3 , which may still fall in the transition regime. Hence Derjaguin and Yalamov's (1972) contention, that these data prove their theory for large particles unambiguously, cannot be accepted. However, for Knudsen numbers greater than 0.75 the data show good agreement with small particle theory.

Stinchcombe and Goldsmith (1966) performed experiments in which iodine was removed from a mixture of steam and air by condensing the steam in a vertical, water-cooled tube. The gas flow was laminar. Part of the iodine was present in gaseous form and part as particulates. They estimated that the particulate removal was 95 to $99 \%$, and attributed this to the effects of diffusio- and thermophoresis, and to inertial and gravitational deposition. However, the latter two mechanisms probably had a minimal influence under their experimental conditions. No theoretical analysis is given. 
Meisen et al. (1971) also worked with the air-water vapour system in a horizontal parallel plate collector. The plates were made of blotting paper held in place by stainless steel wires, the top one being impregnated with steam while the bottom one lay on the surface of a bath of cooling water. Thus a water vapour gradient was established between the plates, and cigarette smoke could be removed continuously from an air stream. The experimental results correlated satisfactorily with schmitt and Waldmann's' theory, as well as the hypothesis that the particles moved at the gas mean mass velocity. This highlights the difficulty of obtaining definitive results when $m_{1}$ and $m_{2}$ are of similar magnitude. Full details of the work are given by Meisen $(1970)$

\subsection{Diffusiophoresis under Turbulent Conditions}

In the first published paper concerning turbulent conditions (Whitmore and Meisen (1973)) the theory of particle transfer was treated analogously to that of mass transfer in turbulent flow. This theory was developed for gas absorption in a wetted wall column, but its wider application was realized. The gas core was assumed to be well mixed with respect to both particles and gas components, and the concentration gradients were assumed to be confined to a thin mass transfer boundary layer. In this way a simple theory could be constructed relating. particle transfer rate to inlet and outlet gas compositions and molecular weights. This theory is presented in 
detail in Chapter 4. Preliminary experimental results were also presented for the ammonia-nitrogen system for large particles, which indicated that the theory was satisfactory, and that the particles moved at approximately the gas mean mass velocity. These results are given in Chapter 6 .

In a later paper (Whitmore and Meisen, to be published in the Canadian Journal of Chemical Engineering) the theory is extended to include diffusiophoresis in multicomponent gases and liquids, and thermophoresis in gases and liquids. Both small and large particles are considered. Relevant material from this paper is given in Chapter 4 .

The only other paper concerning diffusiophoresis under turbulent conditions is that of Azarniouch et al. (1975), which is based on the doctoral work of Azarniouch (1974). The theory presented in this paper is open to several criticisms, and is discussed in detail in Chapter 4 .

Azarniouch passed a mixture of steam and air containing a sulfuric acid mist downwards through a vertical tubular condenser. The mist removal was determined by titrating for sulfuric acid in the condensate. The experimental conditions were such that both inertial deposition and diffusiophoresis were important removal mechanisms. (The contribution of thermophoresis was probably also significant, but it was neglected.) Although Azarniouch attempted to estimate the inertial contribution using a mathematical model, no experimental data are presented for inertial deposition alone. Since deposition models are generally 
imprecise, it is not possible to determine the separate inertial and diffusiophoretic contributions with any accuracy. This difficulty is compounded by the scatter in the data. Another problem is that Azarniouch appears to have misinterpreted his own model by failing to recognize the possibility of interactions between inertial and diffusiophoretic mechanisms, which further complicates the interpretation of the data. This is discussed in Chapter 7 .

Another serious difficulty with the work of Azarniouch et al. concerns the particle size. Although the size of mist aroplets immediately after generation was measured, the size after addition of steam, and the outlet size from the condenser, were not determined experimentally. Instead Azarniouch et al. (1973) made a theoretical study of the growth rate of sulfuric acid droplets at $100^{\circ} \mathrm{C}$ for relative humidities up to 0.3 . They concluded that in their experiments the drops would grow very rapidly (in about $10^{-2}$ seconds) to a stable condition of about double their initial diameter. The particle size at the inlet to the condenser is therefore established, though imprecisely. However, an important factor which has not been considered in their experimental study is the possibility of further droplet growth within the condenser. In regions of high relative humidity which will be present in the condenser, rapid particle growth can be expected according to their earlier theoretical study. It must be concluded that the actual particle size varies with position in the condenser and with experimental conditions, and is unknown. It is thus impossible to determine 
the influence of inertial mechanisms, or to relate the particle size to the mean free path of the gas.

A related paper of interest is that of Byers and Calvert (1969) in which they publish a model for thermophoretic particle deposition from a gas in a tube with constant wall temperature. Unfortunately, their theory does not allow for the effect of temperature variations on gas density, since the density was assumed to be constant. Our theory (Whitmore and Meisen, to be published in the Canadian Journal of Chemical. Engineering) indicates that contraction of the gas upon cooling increases the particle concentration based on unit volume of gas more than it is reduced by particle deposition. It is therefore not surprising that their model does not give very good agreement with their experimental data. Further discussion of this work appears in Chapter 4. Although Azarniouch's (1975) model is the direct mass transfer analogue of Byers and Calvert's (1969) model, he makes no reference to their work.

In a later paper Nishio et al. (1974) derive another model for thermophoretic deposition in a tube with constant wall temperature. The derivation is slightly more direct than that of Byers and Calvert, but the problem of assuming constant fluid density remains. However, the authors do get moderate agreement between their model and their data. No analogue of this model for the case of diffusiophoresis exists. If constructed, however, it should be an improvement on that of Azarniouch et al. The work is further discussed in Chapter 4. 


\subsection{Review Articles}

General reviews of various aspects of diffusiophoresis have been made by Waldmann (1961), Waldmann and Schmitt (1966), Goldsmith and May (1966), Meisen (1970), Derjaguin and Yalamov (1972), and Azarniouch (1974). 


\section{FUNDAMENTAL THEORY}

\subsection{Outline}

The fundamental theory of diffusiophoresis for a large particle in a binary gas mixture undergoing equimolar counterdiffusion is presented in several sections. Firstly, the continuum equations are simplified, subject to certain conditions. Secondly, using these equations, the free particle velocity is determined for an arbitrary slip condition at its surface. Thirdly, the rate of energy dissipation in the gas is calculated in order to deduce the slip condition; and finally, the theory is further refined and extended.

\subsection{The Continuum Mechanics Equations}

The simplified continuum mechanics equations are now developed for the case of a spherical particle suspended in a binary gas mixture. The mixture is considered to undergo equimolar counter-diffusion far away from the particle as indicated in 

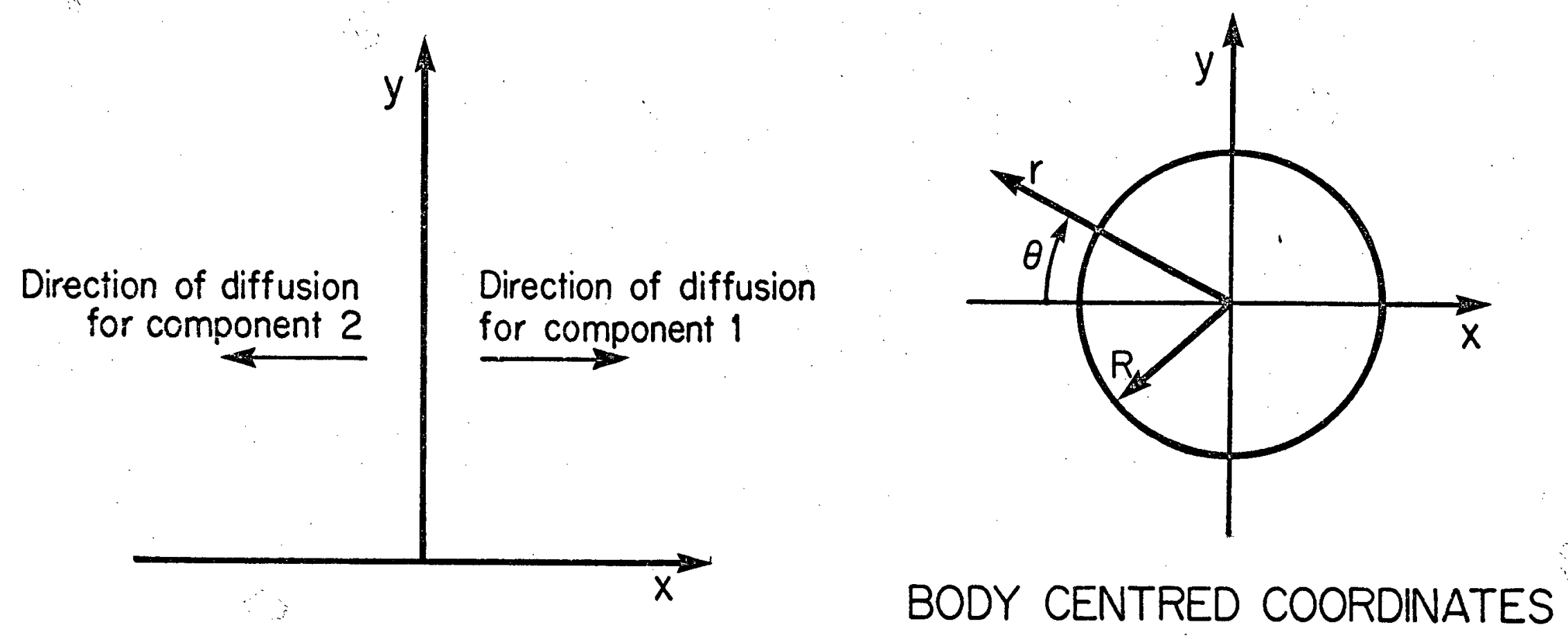

NUMBER CENTRED COORDINATES

Figure 3.1 Coordinate systems. 
Figure 3.1. These equations will be used to find the free particle velocity. Later a simple extension will be given to the case of more practical interest where one gas diffuses through another which is stagnant.

Two types of coordinate systems are needed in the derivation; coordinates with respect to which the mean molar velocity of the gas far from the particle is zero, and coordinates that have their origin at the centre of the particle. For brevity these will be termed "number-centred" and "body-centred" coordinates, respectively.

The behaviour of the fluid in the system is governed by three equations, the Navier-Stokes equation (NS), the total continuity equation (TC), and the partial continuity equation (PC). These ensure conservation of momentum, total mass, and mass of one component respectively.

$$
\begin{aligned}
& -\underline{\nabla} p-\underline{\nabla} \cdot \underline{\tau}+\rho \underline{g}=0 \\
& \frac{\partial \rho}{\partial t}+\underline{\nabla} \cdot \rho \underline{\mathrm{V}}=0 \\
& \frac{\partial \rho_{1}}{\partial t}+\underline{\nabla} \cdot \rho_{1} \underline{v}=\underline{\nabla} \cdot \rho D \underline{\nabla}\left(\frac{\rho_{1}}{\rho}\right) \quad(\mathrm{PC})
\end{aligned}
$$

Since the Reynolds number of particles undergoing diffusiophoresis is small $(<<)$, the inertial terms in the Navier-stokes equation have been ignored.

The partial continuity equation will be solved for 
constant diffusivity, D, and the Navier-Stokes equation for constant viscosity, $\mu$. Since the diffusivity is essentially constant and the viscosity shows little variation in many binary mixtures, these are satisfactory approximations. In any case it is not expected that the predicted particle velocity would be very sensitive to variations in $\mu$ or $D$ over the flow field. The following identities will be used. (These identities and the basic continuum equations can be found for example in BIRD et al. (1960).)

$$
\begin{aligned}
& c_{1}=r_{1} c, c_{2}=r_{2} c, c=c_{1}+c_{2}=\text { constant } \\
& \rho_{1}=M_{1} c_{1}, \rho_{2}=M_{2} c_{2}, \rho=\rho_{1}+\rho_{2}
\end{aligned}
$$

Far from the particle the diffusion is described by the equations,

$$
\mathrm{N}_{1}=-\mathrm{Dc}\left(\frac{\partial \gamma_{1}}{\partial \mathrm{x}}\right)_{\infty}=\mathrm{Dc}\left(\frac{\partial \gamma_{2}}{\partial \mathrm{x}}\right)_{\infty}=-\mathrm{N}_{2}
$$

so that it follows that $\left(\partial \gamma_{2} / \partial x\right)_{\infty}$ is a constant. The mean mass velocity of the gas in this region, with respect to number-centred coordinates, is therefore

$$
w=-\frac{M_{2}-M_{1}}{M} D\left(\frac{\partial \gamma_{2}}{\partial x}\right)_{\infty}=-\frac{D}{\rho}\left(\frac{\partial \rho}{\partial x}\right)_{\infty}
$$

where $\mathrm{M}$ is the mean molecular weight, $\gamma_{1} \mathrm{M}_{1}+\gamma_{2} \mathrm{M}_{2}$. 
The simplification of the Navier-Stokes equation for axisymetrical.: systems with constant fluid density is well known. A similar simplification is now made in the general case. (The subsequent development is similar to that given by Happel and Brenner (1965)). Taking the curl of Equation (3.1) eliminates the gradients of scalars and the $\rho$ g term,giving

$$
\underline{\nabla} \underline{x}(\underline{\nabla} \cdot \underline{\tau})=0
$$

For Newtonian fluids the stress tensor can be written as

$$
\tau=-\mu(\underline{\nabla} \underline{v}+(\underline{\nabla} \underline{v})+)+\left(\frac{2}{3}-k\right)(\underline{\nabla} \cdot \underline{v}) \underline{\delta}
$$

where $(\underline{\nabla} \underline{v})^{+}$is the transpose of $\underline{\nabla} \underline{v}, \mu$ is the shear viscosity, $k$ is the bulk viscosity, and $\delta$ is the unit tensor. The form of the stress tensor is that used by Bird et al. (1960), which does not include the absolute pressure. After some manipulation one can show that

$$
\underline{\nabla} \cdot \underline{\tau}=-\mu\left(\underline{\nabla}^{2} \underline{v}+\underline{\nabla}(\underline{\nabla} \cdot \underline{v})\right)+\underline{\nabla}\left(\frac{2}{3}-k\right)(\underline{\nabla} \cdot \underline{v})
$$

For $\mu$ constant the Navier-stokes equation then reduces to

$$
\underline{\nabla} \underline{x}(\underline{\nabla} \underline{x}(\underline{\nabla} \underline{\times} \underline{v}))=0
$$

which is identical to the form obtained for constant density systems. In that case, however, the equation can be rewritten in 
38.

terms of a single scalar variable, the stream function, so that the flow field is completely determined. Here this is not possible.

The partial continuity equation can be simplified for constant $D$ by eliminating $\rho_{1}$. Using the total continuity to eliminate terms and expanding gives

$$
\underline{\nabla} \cdot \underline{v}=\mathrm{D}\left(\frac{\nabla \rho \cdot \underline{\nabla} \rho}{\rho^{2}}-\frac{\nabla^{2} \rho}{\rho}\right)
$$

To facilitate simplification of the three basic equations they are now recast in dimensionless form. Angular brackets denote dimensionless variables. Hence

$$
\langle v\rangle=\frac{\underline{v}}{w_{0}},\langle\rho\rangle=\frac{\rho}{\rho_{0}},\langle r\rangle=\frac{r}{R},\langle t\rangle=t \frac{w_{O}}{R}
$$

where $\rho_{0}$ and $w_{0}$ are the values of these variables for large $r$ and $\theta=\pi / 2$ in body-centred coordinates. From the expression for $w$ given previously, the following relationship is found, where $n$ is the dimensionless density gradient far from the particle.

$$
n=\left\langle\frac{\partial \rho}{\partial x}\right\rangle_{\infty}=-\frac{{ }^{w_{O}} R}{D}
$$

This equation is only strictly correct when the particle moves at the mean molar velocity. The error involved will be discussed 
later. In dimensionless terms the fluid equations become

$$
\begin{aligned}
& \langle\underline{\nabla} \underline{x}(\underline{\nabla} \underline{x}(\underline{\nabla} \underline{x} \underline{v}))\rangle=0 \\
& \left\langle\frac{\partial \rho}{\partial t}+\underline{\nabla} \cdot \rho \underline{v}\right\rangle=0 \\
& n\langle\underline{\nabla} \cdot \underline{v}\rangle=-\left\langle\frac{\underline{\nabla} \rho \cdot \underline{\nabla} \rho}{\rho^{2}}-\frac{\underline{\nabla}^{2} \rho}{\rho}\right\rangle
\end{aligned}
$$

If the particle moves at other than the mean molar velocity, the value of $\partial \rho / \partial t$ with respect to body-centred coordinates is not zero. As an approximation it may be assumed that a quasi-steady state exists, such that, with respect to the particle, $\partial \rho / \partial t$ is constant everywhere throughout the field. It follows that

$$
\left\langle\frac{\partial \rho}{\partial t}\right\rangle=\left\langle\left(\frac{\partial \rho}{\partial x}\right)_{\infty}\left(\frac{\partial x}{\partial t}\right)_{\infty}\right\rangle=n v_{p}
$$

where $v_{p}$ is the particle velocity with respect to a numbercentred coordinate system. Hence the total continuity equation becomes

$$
\left\langle n v_{p}+\underline{\nabla} \cdot p \underline{v}\right\rangle=0
$$

The behaviour of the system depends on the parameter $\eta$. If $\eta$ is small (as is normally the case in practical situations), further simplification is possible. From the total continuity equation, 


$$
\langle\underline{\nabla} \cdot \underline{v}\rangle=\left\langle\frac{-\underline{v} \cdot \underline{\nabla} \rho-n v_{p}}{\rho}\right\rangle
$$

so that $\langle\underline{\nabla} \cdot \underline{v}\rangle$ is of order $n$. Hence in general $\langle\underline{\nabla} \cdot \underline{v}\rangle<\langle|\underline{v}|\rangle$, and the Navier Stokes equation can be solved with respect to body-centred coordinates tó good approximation by setting $\underline{\nabla} \cdot \underline{v}$ equal to zero. If the particle moved essentially at the mean mass velocity, then $\langle\underline{\nabla} \cdot \underline{v}\rangle$ would not be small everywhere compared to $\langle|\underline{v}|\rangle$. However, in this case velocities in the flow field are of order $n$, so that the error in the predicted particle velocity is still small.

For $\underline{\nabla} \cdot \underline{v}=0$ the Navier-Stokes equation can be rewritten in terms of the stream function $\psi$ (see for example Happel and Brenner (1965)),

$$
E^{2}\left(E^{2} \psi\right)=E^{4} \psi=0
$$

where $\mathrm{E}^{2}$ is the differential operator.

$$
E^{2}=\frac{\partial^{2}}{\partial r^{2}}+\frac{\sin \theta}{r^{2}} \frac{\partial}{\partial \theta}\left(\frac{1}{\sin \theta} \frac{\partial}{\partial \theta}\right)
$$

The velocity components can be found from the stream function.

$$
v_{r}=-\frac{1}{r^{2} \sin \theta} \frac{\partial \psi}{\partial \theta}, \quad v_{\theta}=+\frac{1}{r \sin \theta} \frac{\partial \psi}{\partial r}
$$


A similar simplification can be made for the partial continuity equation, when $\eta$ is small, to yield

$$
\left\langle\underline{\nabla}^{2} \rho\right\rangle=0
$$

This equation can be solved with respect to body-centred coordinates with the boundary conditions

$$
\langle\rho\rangle_{\infty}=\langle 1+n r \cos \theta\rangle,\left(\frac{\partial \rho}{\partial r}\right)_{S}=0
$$

to yield the result

$$
\langle\rho\rangle=\left\langle 1+n\left(r+\frac{1}{2 r^{2}}\right) \cos \theta\right\rangle
$$

Hence, leaving aside non-steady-state aspects, the use of the simple forms of the continuum mechanics equations has been justified on condition that $n$ is small. In particular it is not required that the concentration of one component relative to the other be small, as some workers believed.

\subsection{Determination of the Free Particle Velocity}

The general solution of the stream function for flow around a solid sphere is (see Happel and Brenner (1965))

$$
\langle\psi\rangle=\left\langle\frac{A^{\prime}}{r}+B^{\prime} r+C^{\prime} r^{2}+D^{\prime} r^{4}\right\rangle \sin ^{2} \theta
$$


where $A^{\prime}, B^{\prime}, C^{\prime}$ and $D^{\prime}$ are constants whose values are to be determined. It follows that

$$
\begin{aligned}
& \left\langle v_{r}\right\rangle=-\left\langle\frac{2 A^{\prime}}{r^{3}}+\frac{2 B^{\prime}}{r}+2 C^{\prime}+2 D^{\prime} r^{2}\right\rangle \cos \theta \\
& \left\langle v_{\theta}\right\rangle=+\left\langle\frac{-A^{\prime}}{r^{3}}+\frac{B^{\prime}}{r}+2 C^{\prime}+4 D^{\prime} r^{2}\right\rangle \sin \theta
\end{aligned}
$$

The boundary conditions for large $r$ are given by

$$
\left\langle v_{r}\right\rangle=-\left\langle v_{\infty}\right\rangle \cos \theta,\left\langle v_{\theta}\right\rangle=+\left\langle v_{\infty}\right\rangle \sin \theta,
$$

where $v_{\infty}$ is the fluid velocity with respect to the sphere for large $r$, and is assumed constant. (This condition is discussed later:), It follows that $C^{\prime}=\mathrm{v}_{\infty} / 2$ and $\mathrm{D}^{\prime}=0$, so that

$$
\begin{aligned}
& \left\langle v_{r}\right\rangle=-\left\langle\frac{2 A^{\prime}}{r^{3}}+\frac{2 B^{\prime}}{r}+v_{\infty}\right\rangle \cos \theta \\
& \left\langle v_{\theta}\right\rangle=+\left\langle-\frac{A^{\prime}}{r^{3}}+\frac{B^{\prime}}{r}+v_{\infty}\right\rangle \sin \theta
\end{aligned}
$$

Provided that any slip phenomenon is confined to a region close to the particle surface, it is valid to introduce 
it as a boundary condition. This is the case for a large particle. All proposed expressions for slip at a flat surface have taken the form

$$
v_{\text {slip }}=\alpha D \frac{d \gamma_{2}}{d x}
$$

where $\alpha$ is a slip coefficient usually dependent on $\mathrm{M}_{1}, \mathrm{M}_{2}, \gamma_{1}$ and $\gamma_{2}$. When $n$ is small, $\alpha$ will be a constant to a good approximation. Expressed in terms of dimensionless variables and spherical coordinates, the slip velocity is

$$
\left\langle v_{\text {slip }}\right\rangle=-\frac{\alpha^{\prime}}{\eta}\left\langle\frac{1}{r}\left(\frac{\partial \rho}{\partial \theta}\right)\right\rangle_{s},
$$

where the slip coefficient has been redefined for convenience as

$$
\alpha^{\prime}=\frac{\mathrm{M}}{\mathrm{M}_{2}-\mathrm{M}_{1}} \alpha
$$

Substituting for the density gradient gives the surface boundary condition for $v_{\theta}$, while that for $v_{r}$ is implied by the impermeable particle surface

$$
\left\langle v_{r}\right\rangle=0,\left\langle v_{\theta}\right\rangle=\left\langle v_{s} \operatorname{sip}\right\rangle=+\frac{3}{2} \alpha^{\prime} \sin \theta, \text { at }\langle r\rangle=1
$$


The coefficients $A^{\prime}$ and $B^{\prime}$ can now be determined

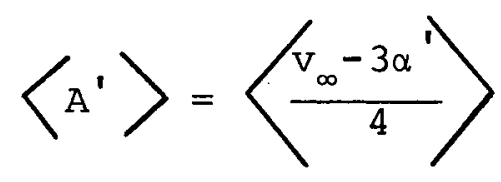

$$
\left\langle B^{\prime}\right\rangle=\left\langle\frac{3\left(\alpha^{\prime}-v_{\infty}\right)}{4}\right\rangle
$$

so that the flow field is solved in terms of $v_{\infty}$ and $\alpha$.

It can be shown (see Happel and Brenner (1965)) that the force on the sphere is given by

$$
F \cdot=+8 \pi \mu \mathrm{w}_{\mathrm{O}} \mathrm{R}\left\langle\mathrm{B}^{\prime}\right\rangle
$$

To find the free particle velocity, the coefficient $B^{\prime}$ is set to zero, so that

$$
\left\langle v_{\infty}\right\rangle=\alpha^{\prime}
$$

or in dimensional terms

$$
v_{\infty}=\alpha D\left(\frac{\partial \gamma_{2}}{\partial x}\right)_{\infty}
$$

Hence the particle velocity with respect to number-centred coordinates is

$$
v_{p}=w-v_{\infty}=\left(-\frac{M_{2}-M_{1}}{M}-\alpha\right) D\left(\frac{\partial \gamma_{2}}{\partial x}\right)_{\infty}
$$


The velocity can now be found for any slip coefficient. For example,Kramers and Kistemaker (1943) give

$$
\alpha=-\left(\frac{M_{2}-M_{1}}{M}-\frac{\sqrt{M_{2}}-\sqrt{M_{1}}}{\gamma_{1}^{\sqrt{M_{1}}+\gamma_{2} \sqrt{M_{2}}}}\right),
$$

so that

$$
v_{p}=-\frac{\sqrt{M_{2}}-\sqrt{M_{1}}}{\gamma_{1} \sqrt{M_{1}}+\gamma_{1} \sqrt{M_{2}}} \quad D\left(\frac{\partial \gamma_{2}}{\partial x}\right)_{\infty} \text {. }
$$

\subsection{The Rate of Energy Dissipation}

The energy dissipation rate, $E$, in the flow field is now calculated for reasons that will become clear shortly. According to Bird et al. (1962) this is given by

$$
E=-\int_{\phi=0}^{2 \pi} \int_{\theta=0}^{\pi} \int_{r=\mathrm{R}}^{\infty}(\tau: \underline{\nabla} \underline{\mathrm{v}}) r^{2} \mathrm{dr} \sin \theta d \theta d \phi
$$

Rewriting the integral in dimensionless form, and inserting the components of $\underline{\tau}: \underline{\nabla} \underline{\mathrm{V}}$ gives 


$$
\begin{aligned}
E & =2 \pi \mu R w_{0}^{2} \int_{0}^{\pi} \int_{R}^{\infty}\left[2\left(\frac{\partial v_{r}}{\partial r}\right)^{2}\right. \\
& +2\left(\frac{1}{r} \frac{\partial v_{\theta}}{\partial \theta}+\frac{v_{r}}{r}\right)^{2}+2\left(\frac{v_{r}}{r}+\frac{v_{\theta} \cot \theta}{r}\right)^{2} \\
& \left.\left.+\left(r \frac{\partial}{\partial r}\left(\frac{v_{\theta}}{r}\right)+\frac{1}{r} \frac{\partial v_{r}}{\partial \theta}\right)^{2}\right] r^{2} \sin \theta d r d \theta\right\rangle .(3.45)
\end{aligned}
$$

substituting for $v_{r}$ and $v_{\theta}$ using Equations (3.31) and (3.32) yields

$$
E=2 \pi \mu \mathrm{Rw}_{\mathrm{O}}^{2}\left\langle 24 \mathrm{~A}^{\prime 2}+16 \mathrm{~A}^{\prime} \mathrm{B}^{\prime}+8 \mathrm{~B}^{\prime 2}\right\rangle
$$

Hence by using the value of $A^{\prime}$ from Equation (3.37), subject to the condition that $B^{\prime}=0$, one finds

$$
E=12 \pi \mu \mathrm{Rw}_{\mathrm{O}}^{2} \alpha^{\prime 2}
$$

It is immediately clear that the rate of energy dissipation is only zero when $\alpha^{\prime}$ is zero. In this case there is no slip and the particle moves at the mean mass velocity, so that with respect to number-centred coordinates, 


$$
v_{p}=-\frac{M_{2}-M_{1}}{M} \quad D\left(\frac{\partial \gamma_{2}}{\partial x}\right)_{\infty}
$$

One can also show that the rate of energy dissipation as given by Equation (3.47) is not generally small, by comparing it with the dissipation rate for a sphere without slip at the surface, and moving with respect to the fluid at the same velocity, $v_{\infty}$, which equals $\alpha^{\prime} \mathrm{w}_{\mathrm{O}}$.

$$
E_{\text {no slip }}=\mathrm{Fv}_{\infty}=6 \pi \mu \mathrm{Rv}_{\infty}^{2}
$$

It follows that $E_{\text {slip }} / E_{\text {no slip }}=2$.

Normally one would expect that the free particle velocity would adjust till the energy dissipation rate were zero. This does not happen here, which raises the question of the source of the energy. The energy dissipation rate in the undisturbed diffusion field is essentially zero, so that energy cannot easily be supplied by the diffusion process. (More precisely, the energy dissipation rate per unit volume in the undisturbed gas is equal to $\frac{4}{3} \mu\left(\frac{w}{R} n\right)^{2}$

Closer examination reveals that the energy originates at the particle surface. The slip condition creates a situation where the gas is being dragged around the surface of the sphere against the force of the resulting shear stresses. Thus a small molecular effect is the source of large quantities of energy: 
It must be concluded that the shear stresses will heavily suppress any tendency for slip to occur, and that the particle will move essentially at the mean mass velocity of the gas. This result is true regardless of particle shape, since the uncoupling of the continuum equations is still valid, and the force on the particle is therefore always zero when it moves at the gas mean mass velocity. The existence of slip at a flat surface, however, is not challenged here. (The author intends to show at a later date that the slip velocity at a flat surface is much smaller than current theories predict. However, this falls beyond the scope of the current study.)

\subsection{Further Refinements and Additions}

\subsubsection{Non-Steady State Behaviour}

The problem has so far been treated as a steady state one, taking the mean mass velocity of the gas to be constant far from the particle. Actually, with respect to number-centred coordinates, this velocity increases in the $\mathrm{x}$ direction at a rate given by

$$
\frac{\partial W}{\partial x}=+\frac{D}{\rho}\left(\frac{\partial \rho}{\partial x}\right)_{\infty}^{2} .
$$

Thus for example, the velocities should strictly not have been made dimensionless with respect to a value of $w$ at fixed body- 
centred coordinates. The true particle velocity in these circumstances is given by a differential equation of the second order with respect to time, and is not of practical interest.. Instead we derive the condition under which the time-dependent effects can be ignored.

One might expect that the particle velocity would always be slightly less than the surrounding gas velocity, which is increasing in the $\mathrm{x}$ direction. This difference will cause the particle to accelerate. Thus, if the particle velocity is fw where $w$ is the gas velocity with respect to number-centred coordinates, and $f$ is a constant close to unity, then according to stokes' law

$$
a_{p}=\frac{F}{M_{p}}=\frac{6 \pi \mu R(w-f w)}{M_{p}},
$$

where $\mathrm{M}_{\mathrm{p}}$ and $\mathrm{a}_{\mathrm{p}}$ denote the particle mass and acceleration, respectively. The fluid acceleration in terms of the same coordinates is

$$
a_{w}=\frac{\partial w}{\partial x} \frac{\partial x}{\partial t}=\frac{D}{\rho}\left(\frac{\partial \rho}{\partial x}\right)_{\infty}^{2} w
$$

For quasi-steady state these accelerations will be approximately equal, from which it follows that 


$$
f \simeq 1-2 / 9 \frac{\rho p}{\rho} \frac{\eta^{2}}{S C}
$$

where $\rho_{\mathrm{p}}$ is the particulate density and Sc is the Schmidt number, $\mu / \rho D$. Since $S C \simeq 1$ for gases, time-dependent effects will be small for

$$
2 / 9 n^{2} \frac{\rho p}{\rho} \ll 1
$$

This criterion is normally met for aerosol particles except when the gas mass transfer rates are extremely high.

\subsubsection{A Second Order Approximation for Particle Velocity}

A second order approximation for the free sphere velocity is now derived, taking into account that the value of $\underline{\nabla} \cdot \underline{V}$ is not exactly zero. According to the total continuity equation

$$
\langle\underline{\nabla} \cdot \underline{v}\rangle=\left\langle-\frac{\underline{v} \cdot \underline{\nabla} \rho}{\rho}-\frac{n v_{p}}{\rho}\right\rangle
$$

If the particle velocity is close to the gas mean mass velocity, then $\langle|\underline{v}|\rangle \simeq n$ and $\left\langle v_{p}\right\rangle \simeq 1$. Thus the first term on the right hand side of Equation (3.55) is much smaller than the second, and 
can be ignored. Hence one obtains

$$
\langle\underline{\nabla} \cdot \underline{v}\rangle \simeq-n
$$

This must be solved in conjunction with

$$
\langle\underline{\nabla} \times \underline{\nabla} \underline{\underline{\nabla}}(\underline{\nabla} \underline{\underline{v}} \underline{\mathrm{v}}))\rangle=0
$$

The velocity components that satisfy these equations are of the form

$$
\begin{aligned}
& \left\langle v_{r}\right\rangle=-\left\langle\left(\frac{2 A^{\prime}}{r^{3}}+\frac{2 B^{\prime}}{r}+v_{\infty}\right) \cos \theta+n\left(r+\frac{G^{\prime}}{r^{2}}\right) \cos ^{2} \theta\right\rangle(3.58) \\
& \left\langle v_{\theta}\right\rangle=+\left\langle\left(\frac{-A^{\prime}}{r^{3}}+\frac{B^{\prime}}{r}+v_{\infty}\right) \sin \theta+n r \cos \theta \sin \theta\right\rangle, \text { (3.59) }
\end{aligned}
$$

where $v_{\infty}$ is the gas velocity past the sphere for large $r$, and $A^{\prime}, B^{\prime}$ and $G^{\prime}$ are constants. Since $v_{r}$ is zero at the surface,

$$
2 \mathrm{~A}^{\prime}+2 \mathrm{~B}^{\prime}+\mathrm{v}_{\infty}=0, \quad \mathrm{G}^{\prime}=-1 .
$$

Also the first term in the expression for $v_{\theta}$ must be zero at the surface if the energy dissipation rate is to be (essentially) zero.

$$
-A^{\prime}+B^{\prime}+V_{\infty}=0
$$


The velocity components are therefore

$$
\begin{aligned}
& \left\langle v_{r}\right\rangle=-\left\langle\left(\frac{1}{2 r^{3}}-\frac{3}{2 r}+1\right) v_{\infty} \cos \theta+n\left(r-\frac{1}{r^{2}}\right) \cos ^{2} \theta\right\rangle(3.62) \\
& \left\langle v_{\theta}\right\rangle=+\left\langle\left(-\frac{1}{4 r^{3}}-\frac{3}{4 r}+1\right) v_{\infty} \sin \theta+n r \cos \theta \sin \theta\right\rangle .(3.63)
\end{aligned}
$$

Note that although it was concluded earlier that there could be essentially no surface slip, the continuum mechanics equations demand that $\left(v_{\dot{\theta}}\right)_{\mathrm{s}}$ does not vanish if $\underline{\nabla} \cdot \underline{\mathrm{v}}$ is not zero. This effect however is small. It is found that the second order terms in the velocity expressions make no contribution to the drag force on the particle. It follows that the flow field around the free particle is given by,

$$
\begin{aligned}
& \left\langle v_{r}\right\rangle=-\left\langle n\left(r-\frac{1}{r^{2}}\right) \cos ^{2} \theta\right\rangle \\
& \left\langle v_{\theta}\right\rangle=+\langle n r \cos \theta \sin \theta\rangle,
\end{aligned}
$$

and that the particle again travels at the gas mean mass velocity as the first order approximation indicated. 


\subsubsection{Particle Velocity in a Binary Gas Mixture with one Stagnant Component}

Lastly, the particle velocity in a system where component 2 of the gas diffuses through component.1, which is stagnant, is derived. The diffusion equations far from the particle are

$$
\mathrm{N}_{2}=-\mathrm{D} \frac{\mathrm{C}}{\gamma_{1}}\left(\frac{\partial \gamma_{2}}{\partial \mathrm{x}}\right)_{\infty}, \quad \mathrm{N}_{1}=0
$$

and the mean molar velocity with respect to the stagnant gas is

$$
\frac{N_{1}+N_{2}}{C}=\frac{-D}{r_{1}}\left(\frac{\partial \gamma_{2}}{\partial x}\right)_{\infty}
$$

With respect to a coordinate system moving at this constant velocity, the gas components exhibit equimolar counter-diffusion. Hence to find the particle velocity in this system one need only add the mean mass velocity with respect to these coordinates, to the velocity of the coordinate system.

$$
v_{p}=-\frac{M_{2}-M_{1}}{M} D\left(\frac{\partial \ddot{\gamma}_{2}}{\partial x}\right)_{\infty}-\frac{D}{\gamma_{1}}\left(\frac{\partial \gamma_{2}}{\partial x}\right)_{\infty}=\frac{M_{2}}{M} \cdot \frac{D}{\gamma_{1}}\left(\frac{\partial \gamma_{2}}{\partial x}\right)_{\infty}
$$

This is simply the mean mass velocity of the gas in the system. 


\subsection{Conclusions}

Provided that the fractional change in fluid density over the length of the particle is small, it is valid to uncouple the continuum mechanics equations. The velocity field around the particle is then given by the Navier stokes equation with $\underline{\nabla} \cdot \underline{v}=0$, and the free particle velocity can be found for any slip condition. The existence of slip, however, is associated with a significant rate of energy dissipation, which appears to exceed the rate at which energy could be supplied in the system. It is therefore believed that the presence of shear stresses at the particle surface must strongly suppress any tendency for slip to occur.

In these circumstances, the particle will move at essentially the gas mean mass velocity, regardless of its shape. This will be true in both equimolar counter-diffusing systems, and systems with one component stagnant. A second order approximation yields the same result. Deviations from the theory caused by accelerative effects, pressure diffusion, and variations in $\mu$ and D will normally be small for aerosol particles. This theory is not in agreement with others proposed recently. Unfortunately.,experimental evidence published to date is inadequate to clarify the situation. 


\section{DERIVED THEORY}

\subsection{The Film Theory}

The theory for the rate of particle deposition by diffusiophoresis can be derived once the fundamental particle velocity is known. In making the derivation, three different velocity expressions will be used. The first is the gas mean molar velocity predicted by Derjaguin et al. (1966) and Derjaguin and Yalamov. (1972), while the second is the gas mean mass velocity predicted in this work. These expressions probably establish the upper and lower limits for the particle velocity in all regimes (molecular, transition, and continuum), since, if one ignores small second-order effects, it is difficult to conceive how a particle could take on a velocity outside this range. The third expression is that obtained by Schmitt and Waldmann (1960) for large particles. This is also the result given by Brock's (1963) work, when second-order terms are ignored and the accommodation coefficients are taken as unity. More importantly, it is the generally accepted expression for small particles under these same conditions, and hence may be useful in determining whether 
experimental results indicate transition regime behaviour.

Since the derivation of the theory does not require any knowledge of equipment geometry, it is presented here for the general case and not specifically for a wetted wall column. Consider a unit of transfer equipment of arbitrary geometry. The turbulent fluid containing suspended particles travels from inlet to outlet by some path (not necessarily linear), which is measured by the $\mathrm{z}$ coordinate. The $\mathrm{x}$ coordinate is taken to be perpendicular to the interface or surface across which transfer occurs. The interfacial or transfer area per unit distance in the direction of fluid flow, a, may be a function of $z$. The following conditions are assumed to hold:

(i) Steady state conditions prevail (at least in a time-averaged sense) and the gas temperature and pressure are constant.

(ii) At any value of the $\mathrm{z}$ coordinate, the bulk of the gas is well mixed with respect to particle and gas concentrations.

(iii) Mass transfer resistance in the gas phase is confined to a thin film adjacent to the transfer surface.

(iv) Mass transfer in the $\mathrm{z}$ direction in the bulk of the gas occurs by convection only, while in the film it is negligible.

(v) The holdup of particles in the film is small. 
(vi) Diffusiophoresis is the only mechanism which transports particles across the film.

(vii) Particles which reach the transfer surface are retained.

(viii) The volume fraction of particles in the gas is small.

These conditions, except for those specifically concerning particles, are either the same as, or analogous to, those invoked in developing the simple theory of mass transfer from turbulent flow (see for example Treybal (1968)).

Although the derivation for multicomponent systems is quite straightforward, for simplicity a binary mixture will be considered in which component 1 is inert and component 2 is transferred. A particle balance over the differential length dz gives

$$
\mathrm{d}(\mathrm{Gn} / \mathrm{c})=-n v_{\mathrm{p}} \mathrm{a} d \mathrm{z} \text {, }
$$

where the left hand side represents the rate of change in the number of particles in the core, and the right hand side represents the rate of transport of particles into the film, and hence their rate of capture. Here $G$ is the molar gas flow rate, $\mathrm{c}$ is the molar gas concentration, $\mathrm{n}$ is the particle number concentration, and $v_{p}$ is the particle velocity in the $x$ direction. Similarly, a mass balance for the gas yields

$$
\mathrm{dG}=-\mathrm{N}_{2} \mathrm{a} \cdot \mathrm{dz},
$$


where $\mathrm{N}_{2}$ is the flux of component 2 into the film. Since component 1 is inert, it is convenient to introduce the particle concentration based on unit volume of this species

$$
\mathrm{n}_{1}=\mathrm{n} / \mathrm{r}_{1}
$$

and the molar flow rate of component 1 which is constant

$$
\mathrm{G}_{1}=\mathrm{G}_{1} \text {, }
$$

where $\gamma_{1}$ is the mole fraction of component 1. The particle and mass balances then become

$$
\mathrm{G}_{1} \mathrm{dn} \mathrm{n}_{1}=-r_{1} \mathrm{n}_{1} \mathrm{cv} \mathrm{p}_{\mathrm{p}} \mathrm{dz}
$$

and

$$
\mathrm{G}_{1} \mathrm{~d}\left(1 / \gamma_{1}\right)=-\mathrm{N}_{2} \mathrm{adz},
$$

since $\mathrm{c}$ is constant. Combining these equations yields

$$
\frac{d n_{1}}{n_{1}}=\frac{r_{1}{ }^{c v_{p}}}{N_{2}} d\left(1 / \gamma_{1}\right) .
$$


If subscripts "in" and "out" denote the terminal conditions, then the particle removal efficiency is obtained by integration.

$$
\varepsilon_{p}=\frac{n_{1, \text { in }}-n_{1, \text { out }}}{n_{1, \text { in }}}=1-\exp \left[\int_{\text {in }}^{\text {out }} \frac{\gamma_{1}{ }^{c v_{p}}}{N_{2}} a\left(1 / \gamma_{1}\right)\right]
$$

This expression can be evaluated once $v_{p}$ and $N_{2}$ are specified. The concentration $\mathrm{n}_{1}$, and the efficiency based on it, are independent of gas composition. Hence the efficiency is influenced only by particle removal, and not by composition changes occurring during mass transfer. This efficiency is identical to the usual one based on the total number of particles entering and leaving the equipment.

An expression for the particle velocity is required for the case when component 2 diffuses through component 1 which is stagnant in the thin film. This expression can be deduced from that applicable to equimolar counter-diffusion, in the manner indicated in Chapter 3. Since the fluxes in the mass transfer film are given by

$$
\mathrm{N}_{1}=0
$$

and

$$
\mathrm{N}_{2}=-\frac{\mathrm{DC}}{\gamma_{1}} \frac{\mathrm{d} \gamma_{2}}{\mathrm{dx}}
$$

where $\mathrm{D}$ is the gas diffusivity, the particle velocity expressions 
can be recast in terms of the flux of component $2^{\dagger}$. There are three cases:

(i) The particles move with the gas mean molar velocity.

$$
v_{p}=-\frac{D}{\gamma_{1}} \frac{d \gamma_{2}}{d x}=\frac{N_{2}}{c}
$$

and hence

$$
\varepsilon_{p}=1-\left[\gamma_{1}\right]_{\text {in }} /\left[\gamma_{1}\right]_{\text {out }}
$$

(ii) The particles move with the gas mean mass velocity.

$$
v_{p}=-\frac{M_{2}}{M} \frac{D}{r_{1}} \frac{d r_{2}}{d x}=\frac{M_{2}}{M} \frac{N_{2}}{C} .
$$

where $\mathrm{M}_{2}$ is the molecular weight of component 2 and $\mathrm{M}$ is the mean molecular weight defined as $\gamma_{1} M_{1}+\gamma_{2} M_{2} \cdot$ Hence

$$
\varepsilon_{\mathrm{p}}=1-\frac{\left[\mathrm{M} / \gamma_{1}\right]_{\text {out }}}{\left[\mathrm{M} / \gamma_{1}\right]_{\text {in }}}
$$

${ }^{\text {Also }}$ the molecule masses $m_{i}$ can be replaced by the molecular weights $\mathrm{M}_{i}$. 
(iii) The particles move with the velocity suggested by Schmitt and Waldmann.

$$
v_{p}=-\frac{\sqrt{\mathrm{M}_{2}}}{\gamma_{1} \sqrt{\mathrm{M}_{1}}+\gamma_{2} \sqrt{\mathrm{M}_{2}}} \frac{\mathrm{D}}{\gamma_{1}} \frac{\mathrm{d} \gamma_{2}}{\mathrm{dx}}=\frac{\sqrt{\mathrm{M}_{2}}}{\gamma_{1} \sqrt{\mathrm{M}_{1}}+\gamma_{2} \sqrt{\mathrm{M}_{2}}} \frac{\mathrm{N}_{2}}{\mathrm{c}}
$$

and hence

$$
\varepsilon_{\mathrm{p}}=1-\frac{\left[\left(\gamma_{1} \sqrt{\mathrm{M}_{1}}+\gamma_{2} \sqrt{\mathrm{M}_{2}}\right) / \gamma_{1}\right]_{\text {out }}}{\left[\left(\gamma_{1} \sqrt{\mathrm{M}_{1}}+\gamma_{2} \sqrt{\mathrm{M}_{2}}\right) / \gamma_{1}\right]_{\text {in }}} .
$$

This derivation is not quite rigorous if the mass transfer boundary layer is partially turbulent, since the expression for the mass transfer flux should be written in terms of the effective diffusivity rather than the molecular diffusivity. It is, however, plausible under these conditions to make the same change in the particle velocity expressions, so that the final results remain unaltered. This work was published by whitmore and Meisen (1973).

\subsection{The General case}

After the film theory was developed, it was found that much more general results could be obtained by analysing the non-steady-state forms of the particle and fluid continuity equations. For this purpose, the fluid continuity 
equation is derived in terms of the velocity adopted by a particle in a diffusing system. The following conditions are required.
(i) The particles move relative to the surrounding fluid only by diffusiophoresis.
(ii) The volume fraction of particles in the fluid is small.

(iii) Particles which reach the transfer surface are retained.

The continuity equation for particles is

$$
\frac{\partial n}{\partial t}+\underline{\nabla} \cdot \underline{n v}_{p}=0
$$

where $\underline{v}_{p}$ must now be interpreted as the particle velocity due to both diffusiophoresis and bulk fluid flow. Again, there are three cases to consider in developing the fluid continuity equations. The basic forms of these equations can be found, for example, in Bird et al. (1960).

\section{(i) The particles move at the fluid mean mass verocity.}

Since the particle velocity equals the mean mass velocity of the fluid, $\underline{v}$, the normal form of the continuity equation for the fluid is required. 


$$
\frac{\partial \rho}{\partial t}+\underline{\nabla} \cdot \underline{\rho} \underline{v}=0
$$

(ii) The particles move at the fluid mean molar velocity.

Written in terms of the mean molar:velocity of the fluid, $v^{*}$, the continuity equation becomes

$$
\frac{\partial \mathrm{C}}{\partial t}+\underline{\nabla} \cdot \underline{\mathrm{CV}}^{*}=0
$$

(iii). The particles move at Schmitt and Waldmann's velocity.

Before developing the appropriate fluid continuity equation, an expression is needed for the particle velocity in a multicomponent gas. Schmitt and Waldmann showed that this velocity is equivalent to that adopted by a free flat surface in contact with a gas mixture whose mean molar velocity is zero, and which is diffusing parallel to the surface. Kramers and Kistemaker (1943) derived this velocity for a binary gas, and by use of their technique it is straightforward to deduce the result for $k$ components 


$$
u_{w}=-\frac{\sum_{i=1}^{k} \sqrt{M_{i}} D_{i} \frac{d \gamma_{i}}{d x}}{\sum_{i=1}^{k} \gamma_{i} \sqrt{M_{i}}},
$$

where $D_{i}$ and $d \gamma_{i} / d x$ are the diffusivity and mole fraction gradient along the surface for component i. The equivalent expression for the case where the mean molar velocity of the mixture, $\mathrm{N} / \mathrm{C}$, is not zero, can be found by addition. This is then also the general expression for particle velocity.

$$
v_{p}=\frac{N}{c}+u_{w}=\frac{N}{c}-\frac{\sum_{i=1}^{k} \sqrt{M_{i}} D_{i} \frac{d \gamma_{i}}{d x}}{\sum_{i=1}^{k} \gamma_{i} \sqrt{M_{i}}}
$$

The expression may be simplified by introducing the molar fluxes of the individual species

$$
N_{i}=\gamma_{i} N-D_{i} C \frac{d \gamma_{i}}{d x},
$$

so that 


$$
\underline{v}_{p}=\frac{\sum_{i=1}^{k} \sqrt{M_{i}} \underline{N}_{i}}{c \sum_{i=1}^{k} \gamma_{i} \sqrt{M_{i}}} .
$$

In order to derive a fluid continuity equation in terms of this type of velocity, a new unit which may be termed the "root mass concentration" is defined:

$$
c_{i}^{r}=\sqrt{M_{i}} \ddot{c}_{i} \text {. }
$$

Other related variables can then be defined to form a compatible system.

$$
\begin{aligned}
& \underline{\underline{N}}^{r}=\underline{N}_{i} \sqrt{\mathrm{M}_{i}} \\
& c^{r}=\sum_{i=1}^{k} c_{i}^{r} \\
& \underline{v}^{r}=\frac{\sum_{i=1}^{k} \underline{N}_{i}^{r}}{c^{r}}
\end{aligned}
$$

The velocity $\underline{v}^{r}$, is now identical to $\underline{v}_{p}$ as required. The fluid continuity equation in terms of these quantities can be derived from the $\mathrm{k}$ single component continuity equations 


$$
\frac{\partial c_{i}}{\partial t}+\underline{\nabla} \cdot \underline{N}_{i}=0
$$

Multiplying each of these by $\sqrt{\mathrm{M}_{i}}$ and summing yields

$$
\frac{\partial}{\partial t}\left(\sum \mathrm{c}_{i} \sqrt{\mathrm{M}_{i}}\right)+\underline{\nabla} \cdot\left(\sum \underline{N}_{i} \sqrt{\mathrm{M}_{i}}\right)=0
$$

Inserting the identities above gives

$$
\frac{\partial c^{r}}{\partial t}+\underline{\nabla} \cdot c^{r} \underline{v}^{r}=0
$$

In each of the three cases the continuity equations for particles and fluid can be written in the following form,

$$
\begin{aligned}
& \frac{\partial \mathrm{n}}{\partial t}+\underline{\nabla} \cdot \underline{n} \underline{v}^{\prime}=0 \\
& \frac{\partial c^{\prime}}{\partial t}+\underline{\nabla} \cdot c^{\prime} \underline{v}^{\prime}=0
\end{aligned}
$$

where $\underline{v}^{\prime}$ is one of the three velocities, and $\mathrm{c}^{\prime}$ is the appropriate related concentration. Multiplying the first equation by $1 / c^{\prime}$ and the second by $\mathrm{n} /\left(\mathrm{c}^{\prime}\right)^{2}$ and subtracting yields 


$$
\frac{\partial\left(n / c^{\prime}\right)}{\partial t}+\underline{v}^{\prime} \cdot \underline{\nabla}\left(n / c^{\prime}\right)=0,
$$

for $c^{\prime} \neq 0$. This can be written as

$$
\frac{D\left(n / C^{\prime}\right)}{D t}=0
$$

where

$$
\frac{\mathrm{D}}{\mathrm{Dt}}=\frac{\partial}{\partial \mathrm{t}}+\underline{\mathrm{v}}^{\prime} \cdot \underline{\nabla}
$$

The quantity $D / D t$ is the time derivative for an element following the fluid as it moves with velocity $\underline{v}^{\prime}$. When $\underline{v}^{\prime}=\underline{v}$, this is equal to the well-known substantial derivative. It follows that either $\mathrm{n} / \mathrm{c}^{\prime}=0$ (trivial solution) or $\mathrm{n} / \mathrm{c}^{\prime}$ is constant.

The particle removal efficiency for an arbitrary piece of equipment can now be determined based on the rate at which particles enter and leave. The quantity $\mathrm{n} / \mathrm{C}^{\prime}$ must be constant throughout the inlet gas. This is true if the inlet gas is well mixed. Hence

$$
\varepsilon_{p}=\frac{\int n v^{\prime} d A-A_{\text {out }}}{A_{\text {in }} \int n v^{\prime} d A}
$$


where $A_{\text {in }}$ and $A_{\text {out }}$ are the inlet and outlet cross-sectional areas, and $\mathrm{v}^{\prime}$ is the component of $\mathrm{v}^{\prime}$ perpendicular to dA. Similarly, the fraction of gas removed measured in appropriate units is

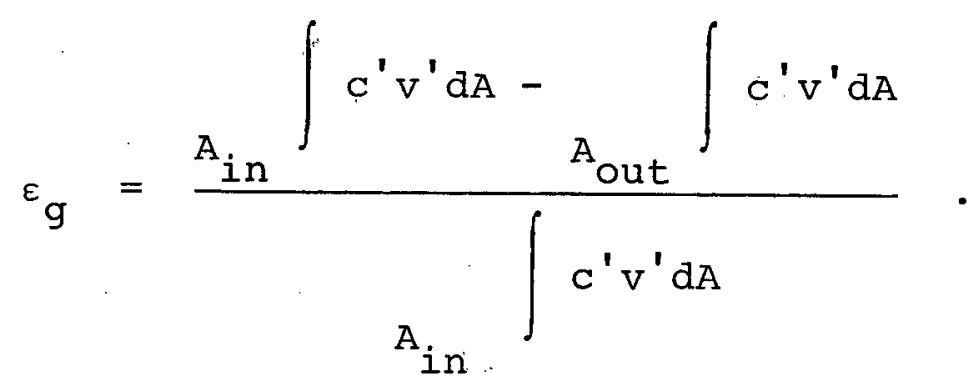

Since $\mathrm{n} / \mathrm{c}^{\prime}$ is constant, it follows that $\varepsilon_{\mathrm{p}}=\varepsilon_{\mathrm{g}}$. Hence

$$
\varepsilon_{p}=1-c^{\prime} \text { out } v^{\prime} \text { out } A_{\text {out }} / C^{\prime} \text { in } v^{\prime} \text { in } A_{\text {in }}
$$

where the subscripted values of $\mathrm{C}^{\prime}$ and $\mathrm{V}^{\prime}$ are appropriate averages for the terminal conditions. However, since component 1 is inert, it follows that

$$
c_{1, \text { in }}^{\prime} v_{\text {in }}^{\prime} A_{\text {in }}=c_{1 \text {,out }}^{\prime} v^{\prime} \text { out }{ }^{A} \text { out } .
$$

Hence

$$
\varepsilon_{p}=1-\left[c^{\prime} / c_{1}^{\prime}\right]_{o u t} /\left[c^{\prime} / c_{l}^{\prime}\right]_{\text {in }} .
$$

This result can now be interpreted for the three cases. 
(i) $\quad \underline{\mathrm{v}}_{\mathrm{p}}=\underline{\mathrm{v}}$.

In this case $\mathrm{c}^{\prime}=\rho$, and it follows that the fraction of particles removed equals the fraction of the total mass of fluid removed. In the case of gases the result can be written as

$$
\varepsilon_{p}=1-\left[M / \gamma_{1}\right]_{\text {out }} /\left[M / \gamma_{I}\right]_{\text {in }} .
$$

(ii) $\quad \underline{\mathrm{v}}_{\mathrm{p}}=\underline{\mathrm{v}}^{*}$.

Here $c^{\prime}=c$, and the general result is that the fractional particle removal equals the fraction of total moles of fluid removed. Since $c$ is constant for gases, a very simple result follows

$$
\varepsilon_{p}=1-\left[\gamma_{1}\right]_{\text {in }} /\left[\gamma_{1}\right]_{\text {out }} \text {. }
$$

(iii) $\quad \underline{v}_{\mathrm{p}}=\underline{\mathrm{v}}^{\mathrm{r}}$.

In this case, $\mathrm{c}^{\prime}=\mathrm{c}^{\mathrm{r}}$, and particle removal efficiency equals the fractional removal of root mass units of fluid. The result for gases is 


$$
\varepsilon_{p}=1-\left[\left(\sum_{i=1}^{k} \gamma_{i} \sqrt{M_{i}}\right) / \gamma_{1}\right]_{\text {out }} /\left[\left(\sum_{i=1}^{k} \gamma_{i} \sqrt{M_{i}}\right) / \gamma_{I}\right]_{i n} .
$$

The above expressions are valid provided that the net transfer expressed in appropriate units is always directed out of the fluid at the transfer surface.

In its more general form, the schmitt and waldmann type velocity expression includes $\mathrm{k}$ coefficients, $a_{i}$, whose value depends on the interaction of the molecules with the particle surface, and on the Knudsen number.

$$
\underline{v}_{p}=\frac{\sum_{i=1}^{k} a_{i} \sqrt{M_{i}}-_{i}}{c \sum_{i=1}^{k} \gamma_{i} a_{i} \sqrt{M_{i}}}
$$

Proceeding as before, but defining ${c_{i}}^{r}$ slightly differently as

$$
c_{i}^{r}=a_{i} \sqrt{M_{i}} c_{i},
$$

one can show that for gases

$$
\varepsilon_{p}=1-\left[\left(\sum_{i=1}^{k} \gamma_{i} a_{i} \sqrt{M_{i}}\right) / \gamma_{1}\right]_{\text {out }} /\left[\left(\sum_{i=1}^{k} \gamma_{i} a_{i} \sqrt{M_{i}}\right) / \gamma_{1}\right]_{i n} \text {. }
$$




\subsection{Discussion of Theory}

The derivations based on the non-steady state forms of the fluid and particle equations are quite general for any fluid under conditions normally encountered in equipment operation, since they follow from simple conservation laws. In particular, they hold whether the fluid is laminar or turbulent. Their application in the laminar case could lead to new and improved methods of measuring diffusiophoretic particle velocity.

The only published paper on diffusiophoresis under turbulent conditions by other authors is that of Azarniouch et al. (1975). Their theory, which resembles that of Whitmore and Meisen (1973) in its general approach, can be criticised in several respects. Their differential mass and particle balances are correct only for dilute systems $\left(\gamma_{2} \approx 0.1\right)$. Even though it is known that higher concentrations can result in significant error, the authors applied their derived expressions to situations where $\gamma_{2}$ exceeded 0.6 .

Azarniouch et al. assumed that mass transfer took place across a laminar film. The concentration gradient in the film was taken to be linear, even though this is true only for dilute systems. The magnitude of the gradient was then determined from the film thickness using the method employed earlier by Byers and Calvert (1969) in their theory of thermophoeretic deposition from turbulent flow. In this method, the thickness of the mass transfer film is found by equating it to the momentum transfer boundary layer thickness, and expressing the latter in terms of hydrodynamic variables. It would have been more direct to deduce 
the thickness of the mass transfer boundary layer from the mass transfer coefficient. Nishio et al. (1974) adopted this approach for heat transfer, and were able to improve on Byers' and Calvert's theory.

Once the gradient was determined, the diffusiophoretic velocity in the boundary layer could be calculated, and the differential particle balance equation integrated over the length of the apparatus to give the removal efficiency. The theory requires that the concentration of the diffusing species at the transfer surface be known, and the integration is not straightforward unless this concentration has a constant value. The final result of Azarniouch et al. is more complex than that of Whitmore and Meisen (1973), even when written in non-predictive form (i.e., in a form dependent on the gas inlet and outlet conditions), while being more limited in its application, and less rigorous in its derivation. 
Chapter 5

\section{EXPERIMENTAL APPARATUS AND PROCEDURE}

\subsection{General}

The apparatus can be divided into four basic parts, of which the wetted wall column is the central one and the others are peripheral. In the experimental work an aerosol generator was used to produce a stream of insoluble (inert) particle-bearing gas. This stream was then mixed with a stream of soluble (transferred) gas to give the desired gas composition. The resulting mixture was passed up through the wetted wall column counter-current to: the water flow. Part of the gas was thus absorbed, and a fraction of the particles removed from the gas and trapped in the water. The degree of particle removal was gauged by measuring the particle concentrations in the inlet and outlet gas streams of the column using an aerosol particle counter. The change in gas composition in the column was determined using an Orsat apparatus. The particle removal efficiency could then be related to this change in gas composition. A general view of the equipment appears in Figure 5.1, and a closeup of the columns in Figure 5.2. Details of purchased equipment is given in Table I, 
74 .

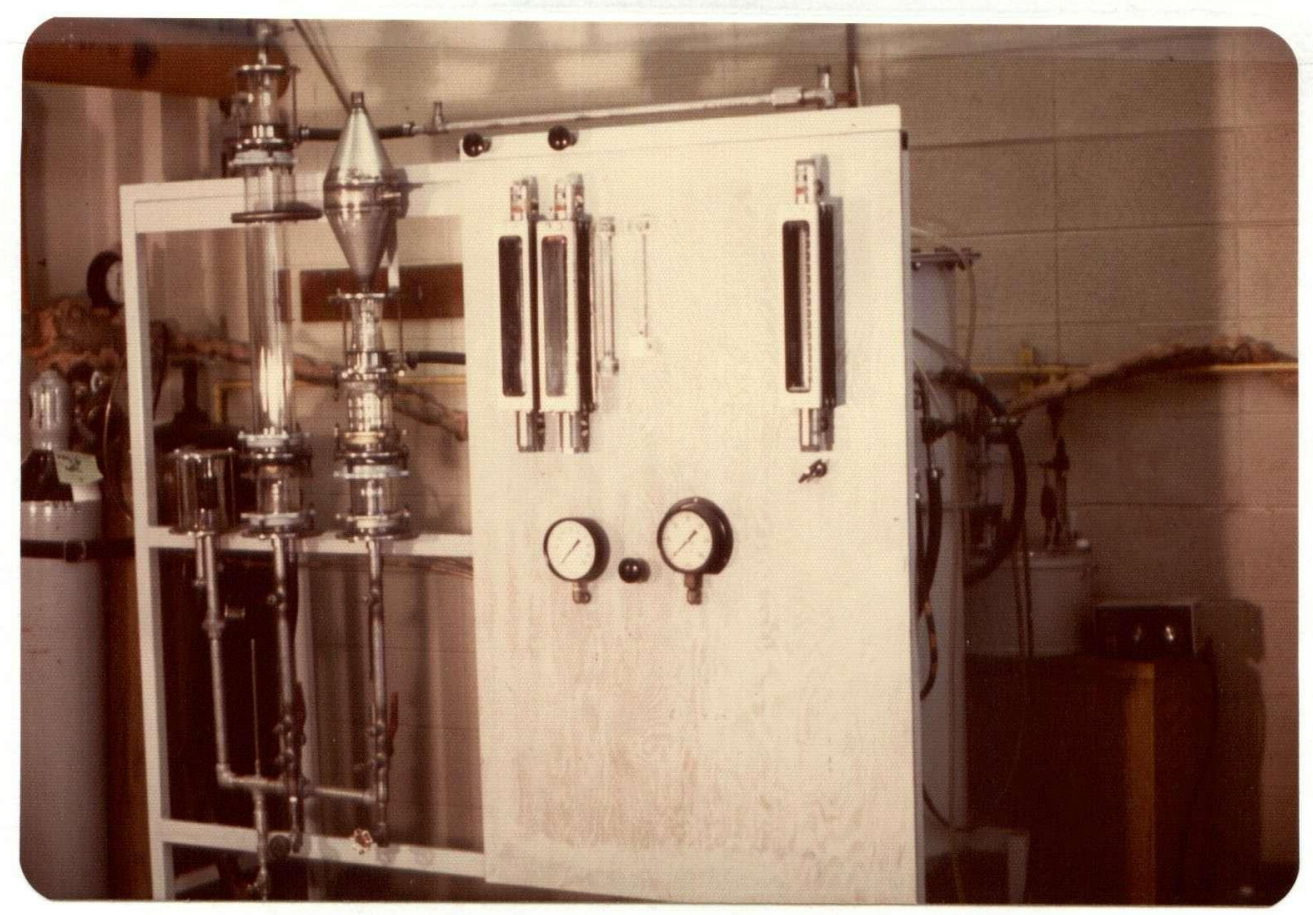

Figure 5.1 General view of the equipment. 


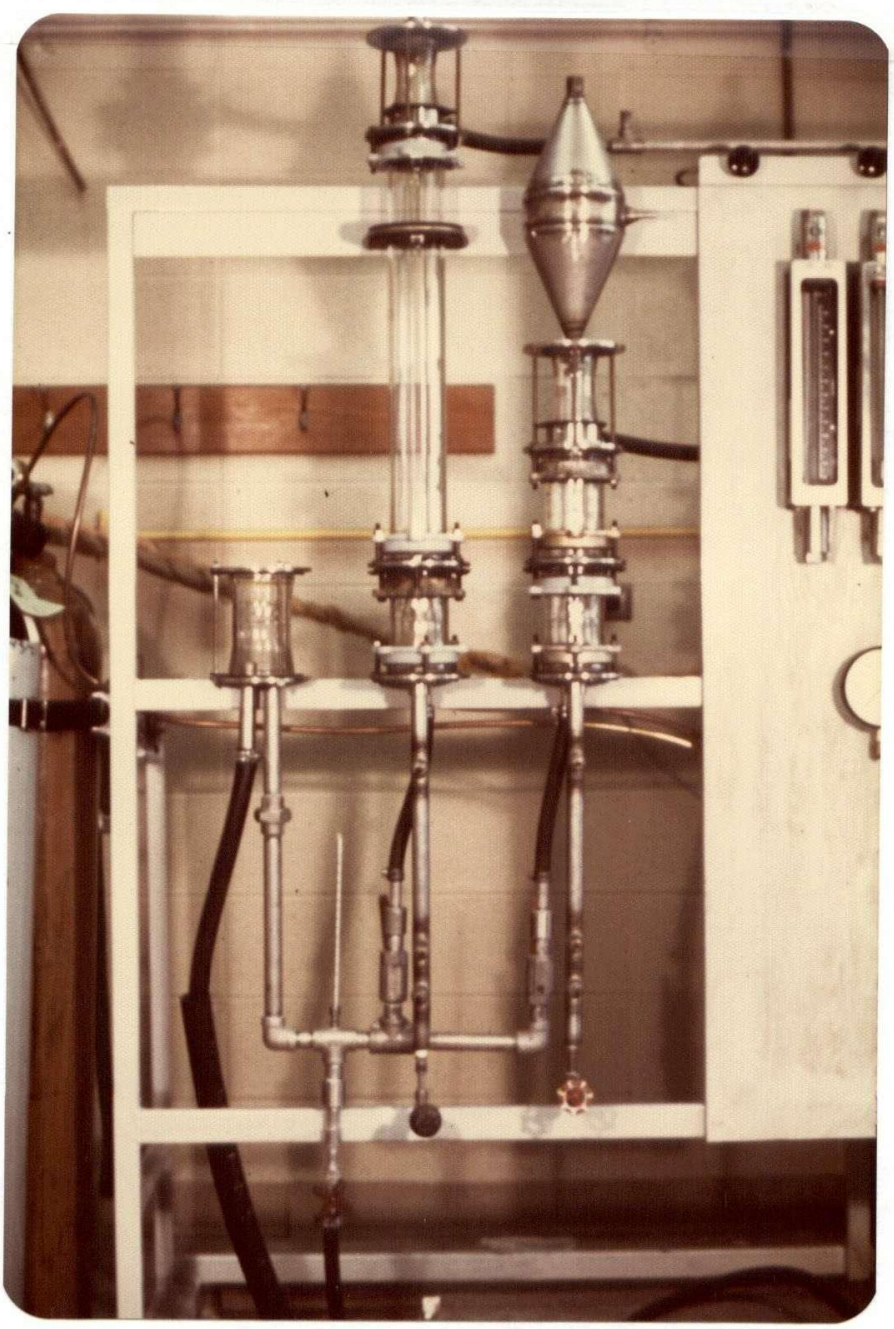

Figure 5.2 Closeup view of the wetted wall columns. 
Table I

Purchased Equipment

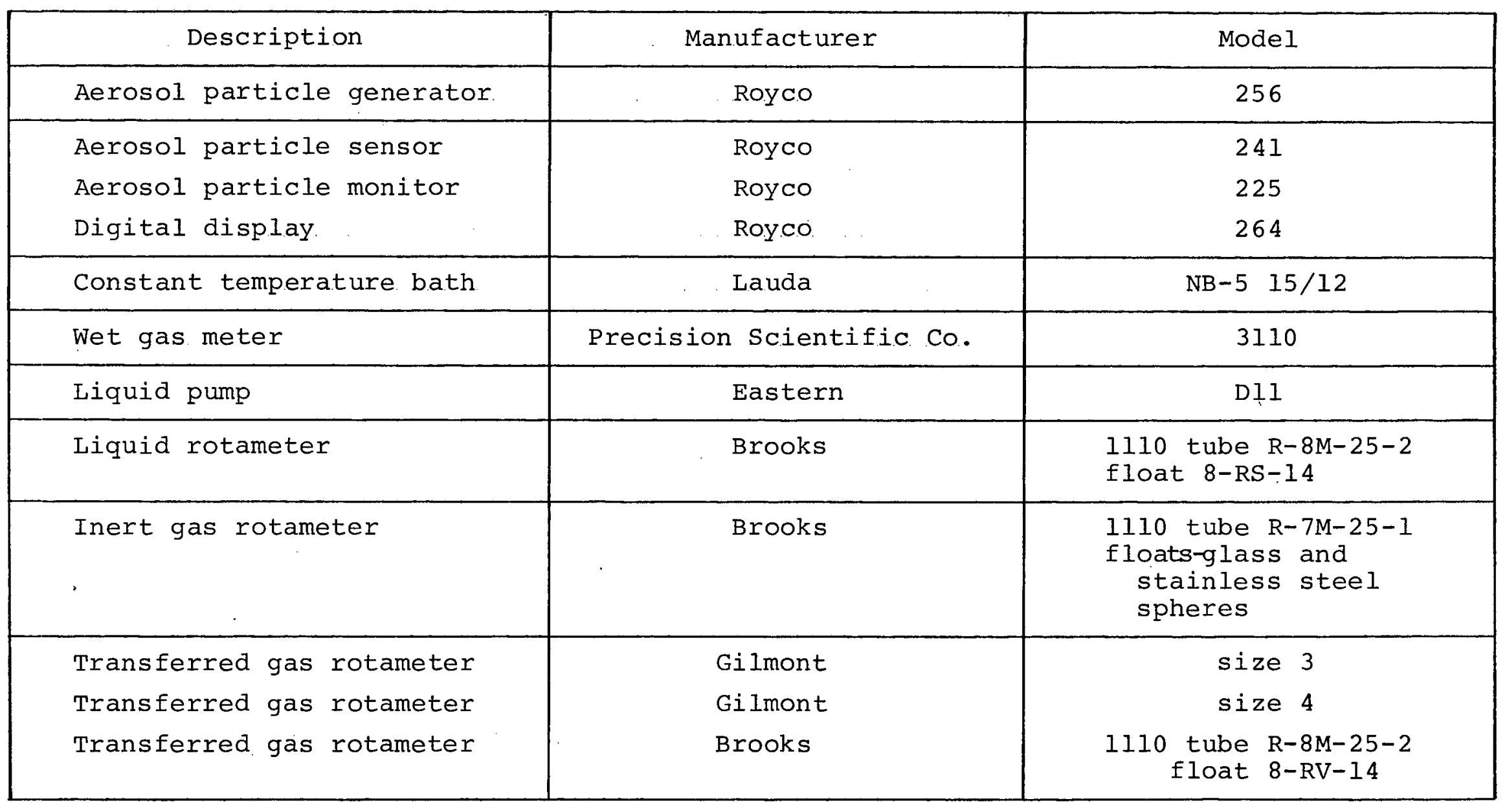


Table II

Gases and Particles

\begin{tabular}{|c|c|c|c|}
\hline Gas & Supplier & Grade & Purity \\
\hline $\begin{array}{l}\text { ammonia } \\
\text { argon } \\
\text { freon } 12 \\
\text { helium } \\
\text { methane } \\
\text { nitrogen } \\
\text { trimethylamine }\end{array}$ & $\begin{array}{l}\text { Canadian Industries Ltd. } \\
\text { Canadian Liquid Air } \\
\text { Matheson } \\
\text { Canadian Liquid Air } \\
\text { Matheson } \\
\text { Canadian Liquid Air } \\
\text { Matheson }\end{array}$ & $\begin{array}{l}\text { anhydrous } \\
\text { standard } \\
\text { standard } \\
\text { standard } \\
\text { technical } \\
\text { g grade } \\
\text { standard }\end{array}$ & $\begin{array}{l}99.995 \% \text { min. } \\
99.995 \% \text { min. } \\
99 \% \text { min. } \\
99.995 \% \text { min. } \\
98 \% \text { min. } \\
99.5 \% \text { min. } \\
99 \% \min .\end{array}$ \\
\hline $\begin{array}{l}\text { Particle } \\
\text { Diameter } \\
\quad(\mu \mathrm{m})\end{array}$ & Supplier & Material & $\begin{array}{c}\text { Standard } \\
\text { Deviation } \\
(\mu \mathrm{m})\end{array}$ \\
\hline
\end{tabular}


and of the gases and particles used in Table II.

\subsection{Apparatus}

5.2.1 The Absorption System .

The initial choice of liquid absorbents rather than solid ab-or adsorbents has been discussed in Chapter 1. Water vapour was the most obvious choice for the transferred gas because of its low cost, potential industrial applicability, and its use by other workers in studies of diffusiophoresis under laminar gas conditions. It could be absorbed in a regenerable liquid such as lithium chloride solution.

There were however two problems with this system. Firstly, mass transfer calculations indicated that the liquid phase resistance would not be negligible, and thus the equilibrium conditions at the liquid surface would not be known. (However, the theory developed later, and described in Chapter 4, showed that a knowledge of gas composition at the surface was not necessary in determining particle removal rates!) Use of stronger absorbents such as concentrated sulfuric acid would reduce the liquid phase resistance, but they would be difficult to handle, and their high heats of absorption would make it more difficult to approximate isothermal absorption in the column. Secondly, significant particle removal would require high vapour concentrations in the inlet gas, which in turn implied temperatures of the order of $80^{\circ} \mathrm{C}$. This would introduce the difficult experimental 
problem of avoiding mist formation in the apparatus and gas lines. (The particle counter purchased later operates only up to $50^{\circ} \mathrm{C}$. ) The use of water vapour systems was therefore ruled out. Several other gases which are easily absorbed were considered. Of these, ammonia and carbon dioxide appeared the most suitable because of their low toxicity and corrosiveness, low cost, and common availability. The column was therefore designed for transfer of carbon dioxide into sodium hydroxide solutions. Since the design methods for gas absorption with chemical reaction are very unreliable, it was recognized that there was a reasonable possibility of inadequate equipment performance. However, calculations indicated that high absorption rates would be achievable with ammonia, and satisfactory equipment performance should result.

\subsubsection{Wetted Wall Column Design.}

The design calculations for mass transfer with chemical reaction followed the methods of Dankwerts (1970). Values for the gas side mass transfer coefficients were estimated from the correlation of Sherwood and Gilliland (1934), while liquid side coefficients were taken from Sherwood and Pigford (1952). Data on flooding were taken from Wallis (1969). Other information came from Treybal (1968) and Perry (1963). Physical properties for the liquid and gas were taken from Perry (1963), International Critical Tables (1926), Handbook of Physics and Chemistry (1970), and Dankwerts (1970). 
The flow rate of the liquid was chosen so as to give turbulence, but even so calculations showed that a high concentration of $\mathrm{NaOH}$ ( $32 \mathrm{w} \%$ ) was necessary to keep the liquid side mass transfer resistance small. The gas rate was set so that the inert gas, if present on its own, would exhibit turbulent flow with respect to the dry column wall. The calculated column height was increased by approximately $50 \%$ in the final design. Further increase was limited by the construction materials chosen. Details of the design are given in Table III.

Essential features of the column design are shown in Figures 5.3 and 5.4. The column was a 1" ID precision bore glass tube supported by 0 rings. It was enclosed by a 3" nom. D glass pipe section to provide protection, mechanical rigidity, and water jacketing if required. The liquid distributor and collector were designed to minimize the exposed liquid surface area while allowing even, droplet-free flow. The gas inlet and outlet tubes were constructed of $1 "$ tubing which matched the column diameter, and were equipped with sampling points. The inlet tube was also designed to act as a mixing chamber for the particle-bearing stream and the remainder of the gas. A second column of identical design, except for its shorter length, was also constructed so as to allow correction for end effects if desired. 
Table III

Wetted Wall Column Design Parameters

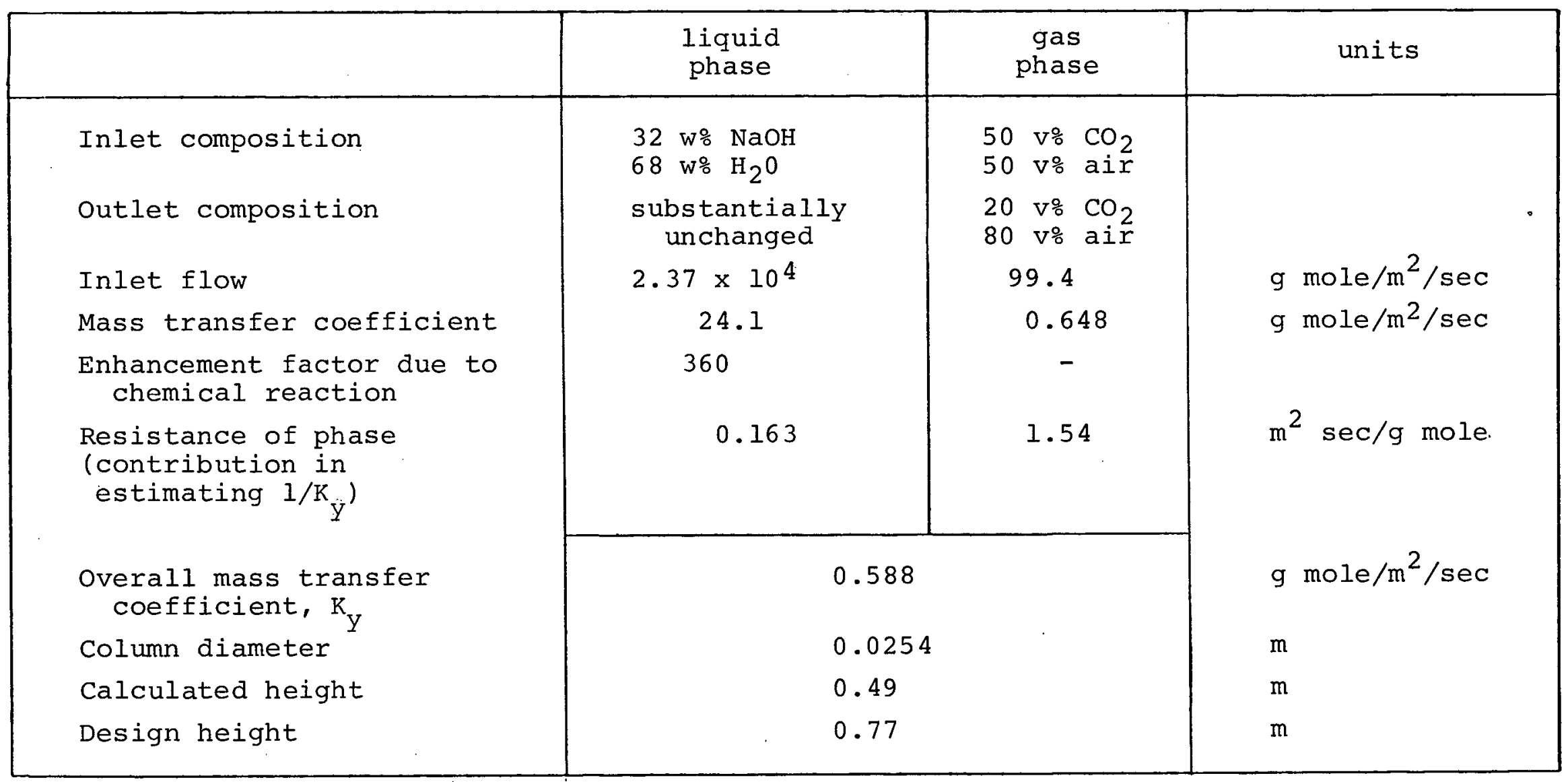




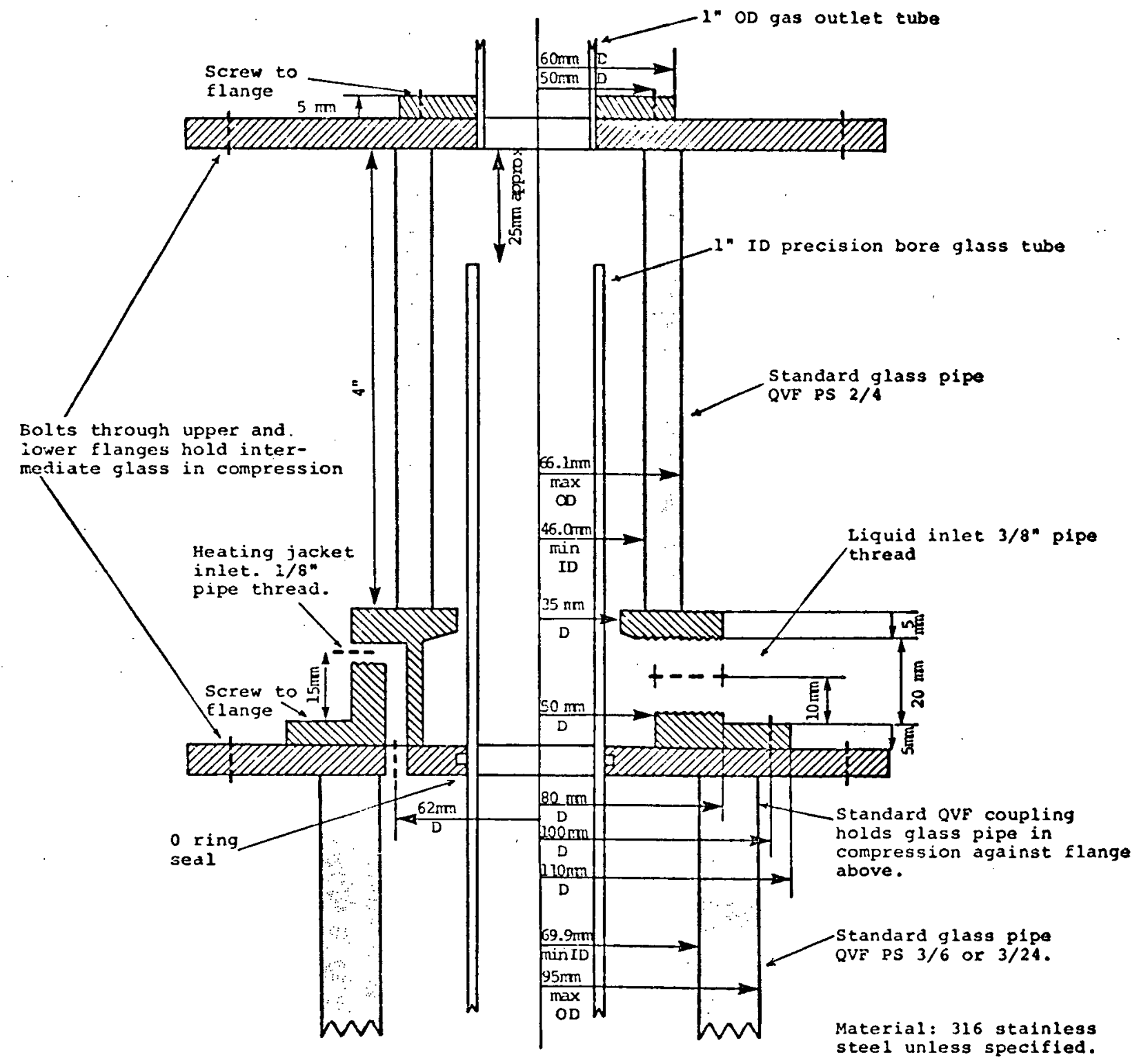

Figure 5.3 Wetted wall column - top. 


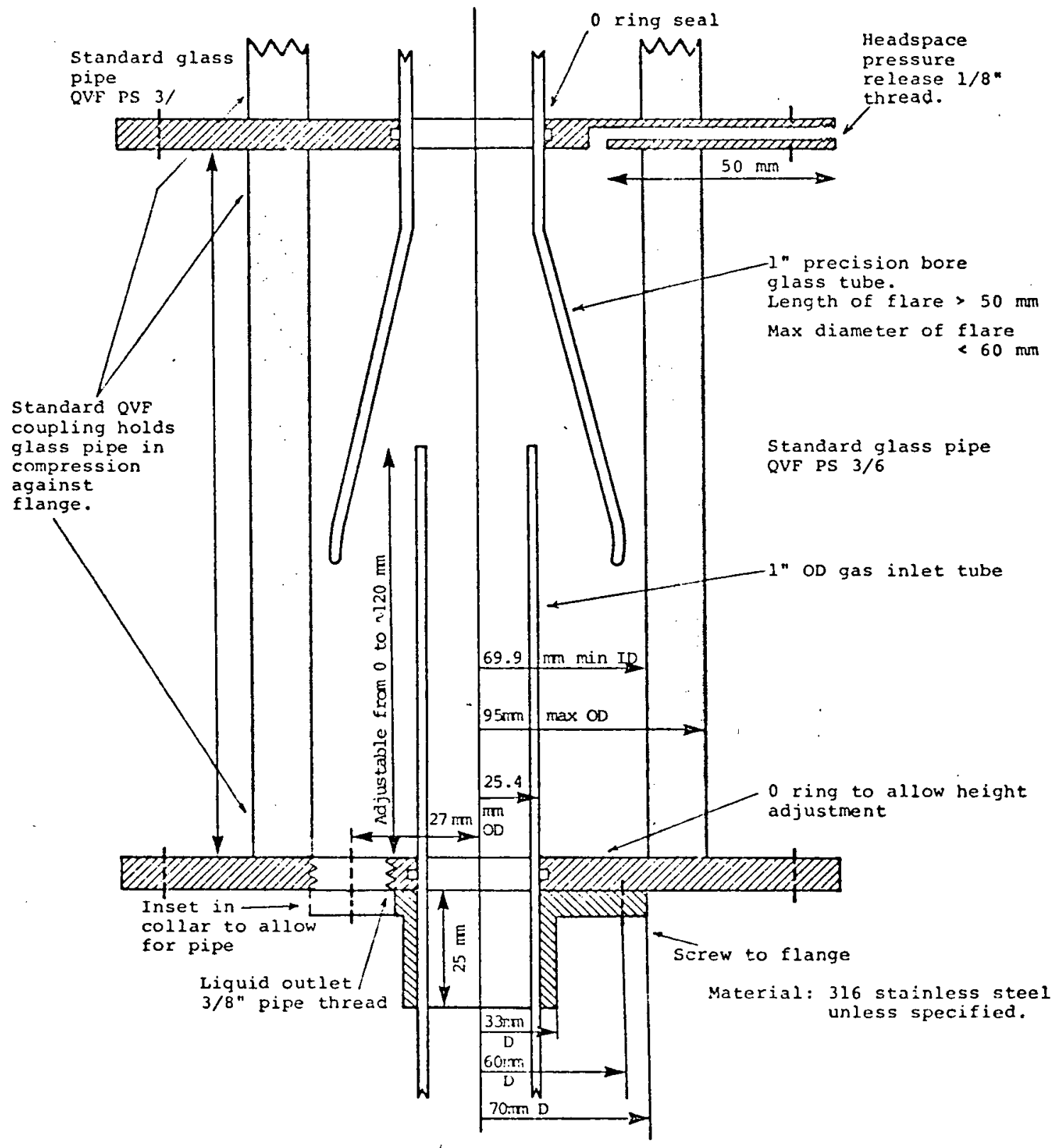

Figure 5.4 Wetted wall column-base. 


\subsubsection{Gas and Liquid Supply Systems.}

The design of the liquid and gas supply systems is shown in Figure 5.5 .

The inert gas was supplied from a cylinder and metered by a rotameter under pressure (normally 50 psig). The flow was then divided, with part going to the aerosol generator. This unit required an inlet pressure of about 30 psig or greater. The remainder was throttled down in pressure and mixed with the transferred gas before being passed to the column. The transferred gas, which was also supplied from a cylinder, was throttled through a control valve before being metered by a rotameter and mixed with the clean inert stream ready for passage to the column. Both inert and transferred gas streams were equilibrated to the liquid supply temperature by being passed through temperature control coils immersed in the liquid storage tank. The gas mixture leaving the column was exhausted to the atmosphere. The liquid was supplied from a tank of approximately 180 litres capacity. Water from a circulating temperature bath passed through coils immersed in the liquid in order to maintain the desired temperature. The liquid was pumped from the tank through a control valve and metering rotameter to the top of the column. Liquid leaving the column passed through a constant head tank, which controlled the level of the liquid, pool at the base of the column, and was then discharged to the drain. 


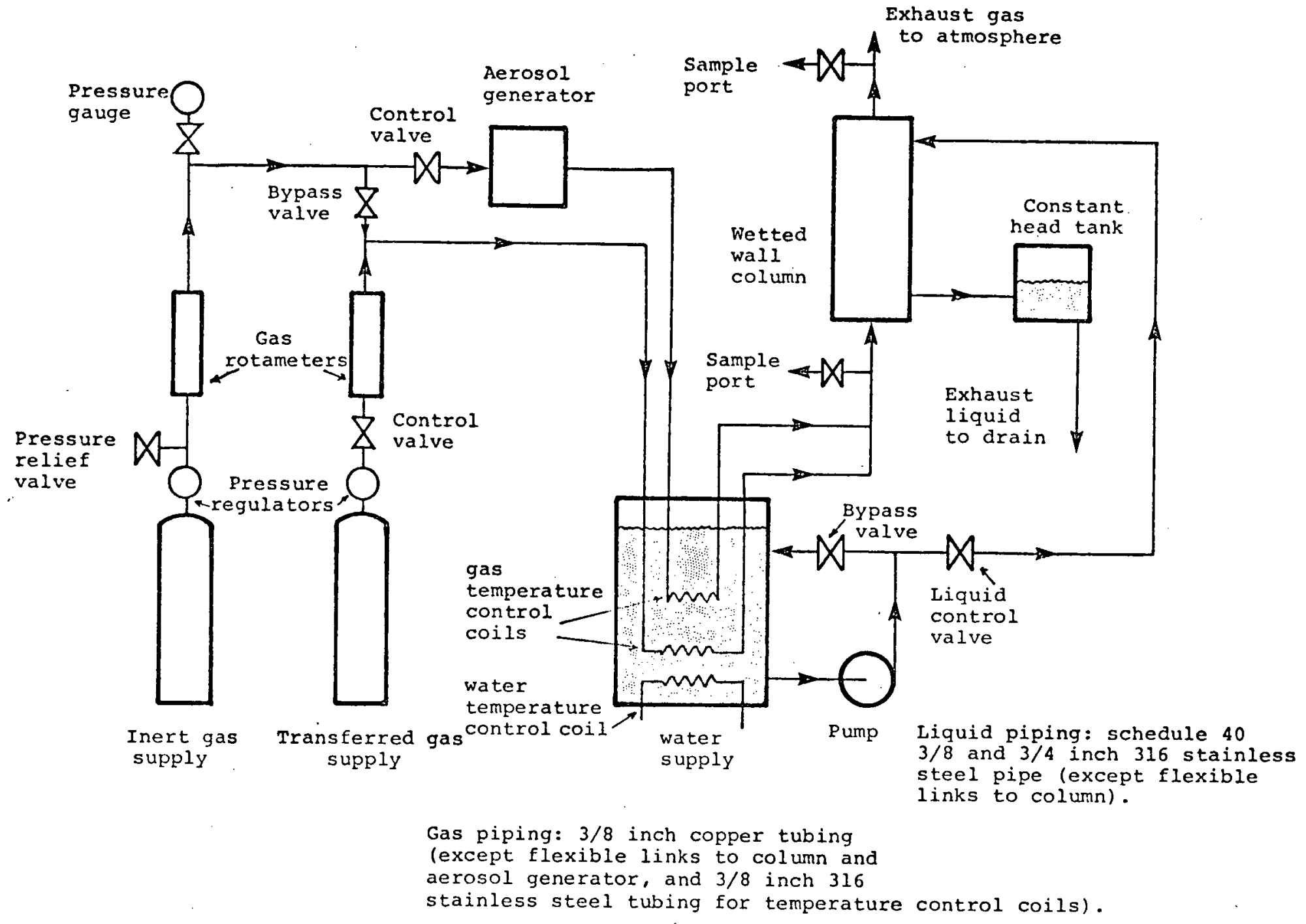

Figure 5.5 Gas and liquid supply systems. (Only one column shown.) 


\subsubsection{Aerosol Generation.}

In the aerosol generator a dilute suspension of uniformly sized latex or polymer spheres in distilled water was atomized in a jet of gas. The resulting droplets were then evaporated by addition of further dry gas, leaving only the spheres. This type of generation was advantageous since it produced a monodisperse aerosol. The effect of particle diameter could be easily investigated by the use of several different sizes of sphere. Electron micrographs of the particles used are shown in Figures 5.6 to 5.10 .

\subsubsection{Aerosol Particle Counter.}

Aerosol concentrations at the column inlet and outlet were determined by withdrawing and analysing sample streams with the particle counter. This instrument used a photomultiplier tube to detect individual particles by forward light scattering. The magnitude of the resulting electrical impulses increased with particle size. Particles greater than $1.2 \mu \mathrm{m}$ D were counted on one channel, and those smaller on another. The instrument detected particles as small as approximately $0.3 \mu \mathrm{m}$ D. Particle concentrations were kept below $03,000,000 / \mathrm{m}^{3}$. At this level co-incidence errors due to two particles passing through the sensitive volume simultaneously become significant. The maximum sample temperature that can be tolerated by the particle counter is $50^{\circ} \mathrm{C}$. 


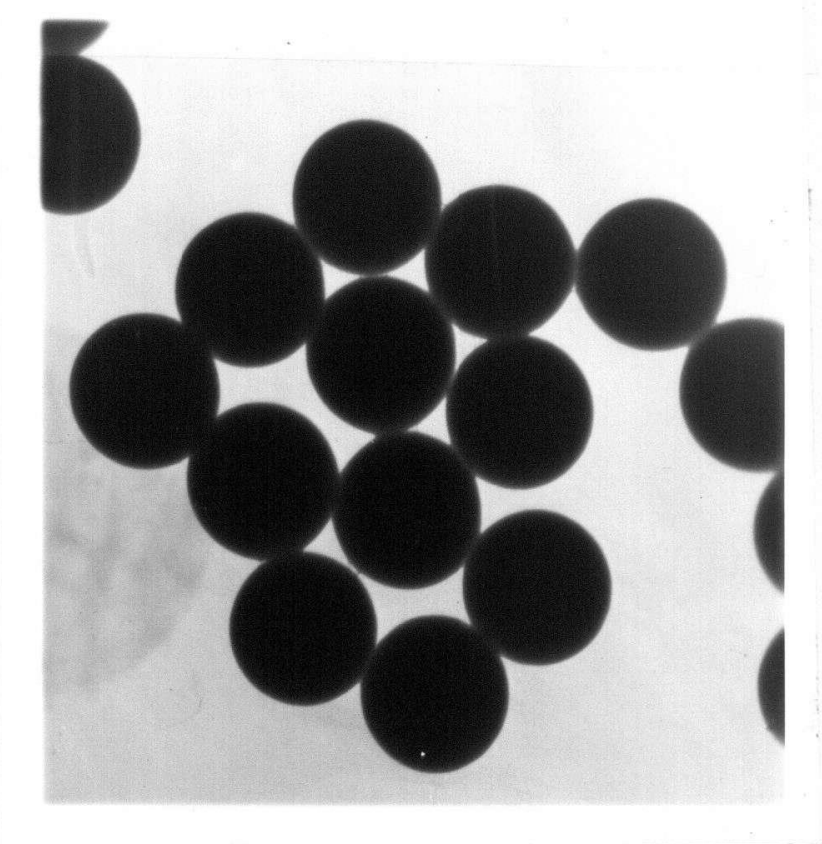

Figure 5.6 Electron micrograph of 0.50 micron diameter latex particles. Magnification $30,000 \mathrm{x}$.

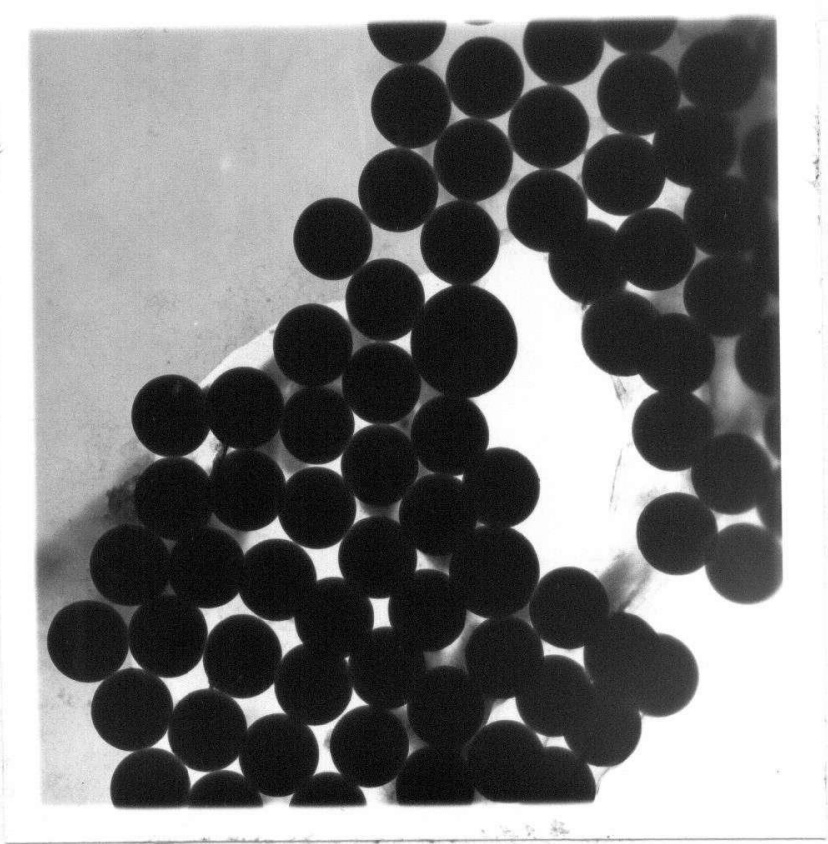

Figure 5.7 Electron micrograph of 0.79 micron diameter latex particles. Magnification $10,000 \mathrm{x}$. 


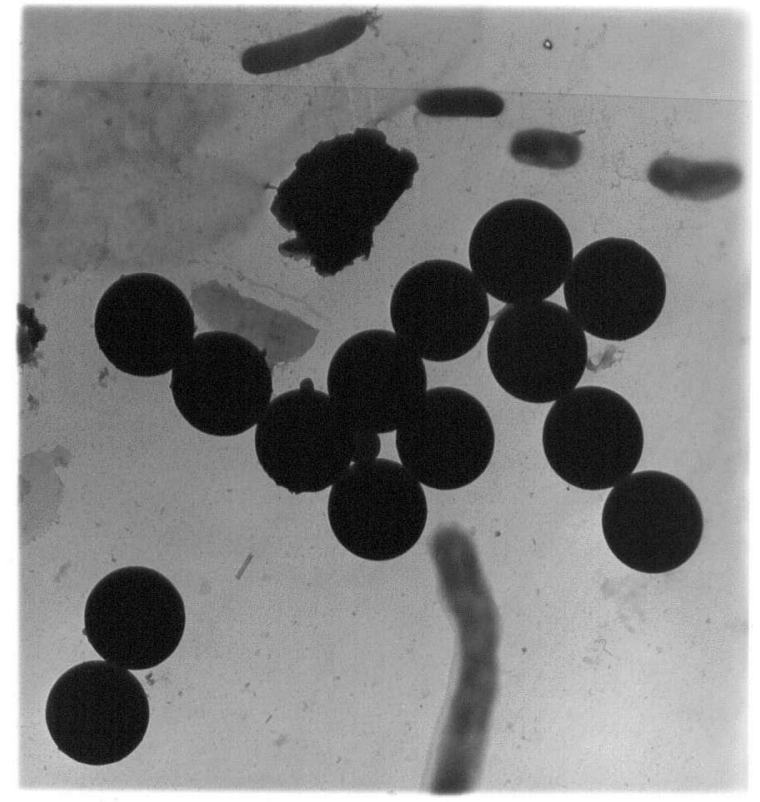

Figure 5.8 Electron micrograph of 1.011 micron diameter latex particles. Magnification $10,000 \mathrm{X}$.

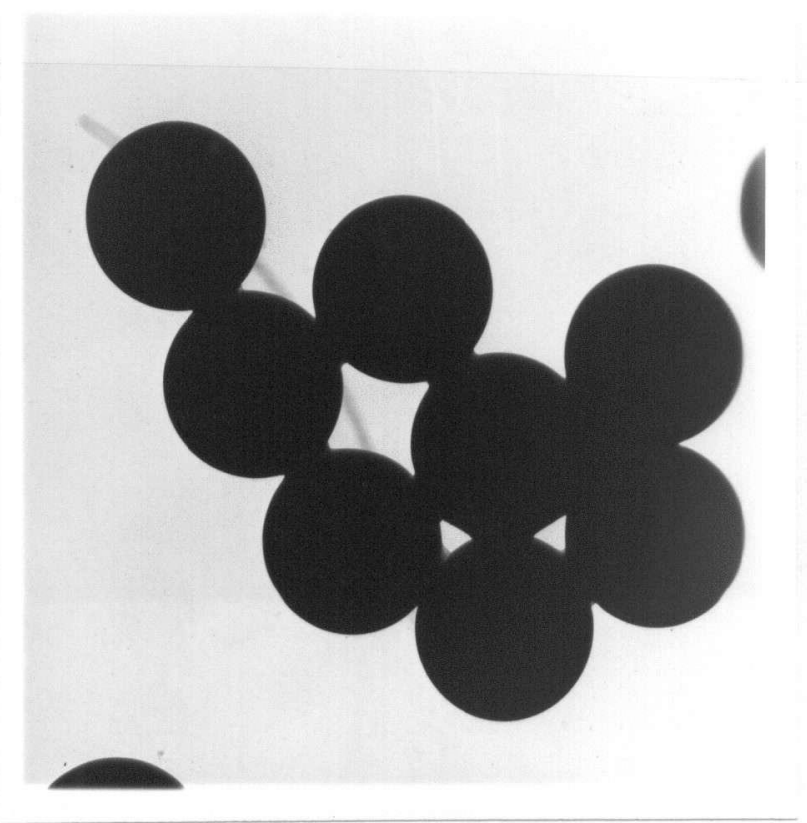

Figure 5.9 Electron micrograph of 2.02 micron diameter latex particles. Magnification $10,000 \mathrm{x}$. 


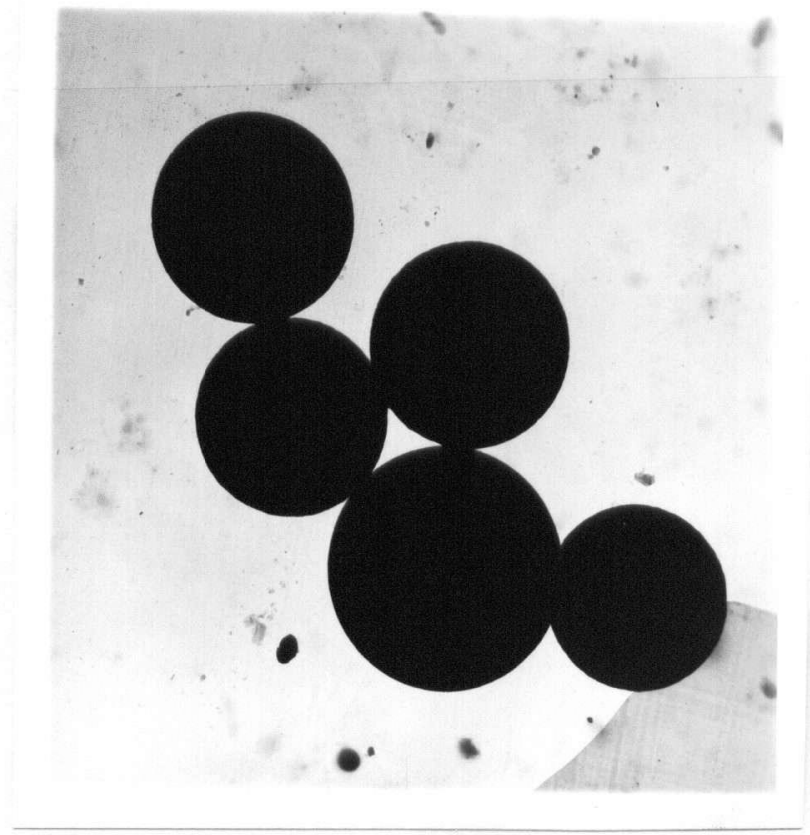

Figure 5.10 Electron micrograph of 5.7 micron diameter latex particles. Magnification 3,000 X. 
Three modifications were made to the counter as purchased. First, the light pipe in the sensor, which was made of "Lexan" (a polycarbonate), was attacked by ammonia during early experimental work till the counter finally became inoperable. A replacement glass fibre optics light pipe has been completely satisfactory. Second, the counter was designed to draw 0.1 cubic feet of air per minute $\left(2.8 \times 10^{-3} \mathrm{~m}^{3} / \mathrm{min}\right)$ at a uniform rate so that the particle concentration could be determined by counting for a set time. However, the flow rate changed substantially when gases of different composition were used. This was to be expected, since the gas recycle rate within the counter and the gas exhaust rate from the counter were controlled by small orifices, making the flow rates dependent on the gas molecular weight. For this reason, modifications were made to the counter so that the gas could be collected from the exhaust orifice. It was then passed through a scrubber system and the remaining inert gas flow measured with a wet gas meter. By this means the count per unit volume of inert gas could be established.

Initially the scrubber consisted of three glass flasks in series, each of approximately $2000 \mathrm{ml}$ capacity and partially filled with concentrated sulfuric acid. Although this system was very effective, early experiments showed that the acid surface tended to cake up. Also, crystalline deposits formed in entrance and exit tubes, inhibiting gas flows and giving erroneous readings. A preliminary scrubber was therefore added, in which the gas flow was contacted with flowing water in a glass flask, removing most of the soluble gas. The water flow was kept 
low enough that the quantities of dissolved air released from the water, and of inert gas dissolving into it, were negligible. Finally, the "Gast" vacuum pump model 1531, used to draw the sample through the counter, was designed to purge a small amount of gas out of the pump chamber through the bearing. This prevented dirt and oil entering the pump chamber and interfering with the movement of the carbon vanes. By rearranging the bearing seals and drilling a small hole into the cavity near the bearing, it was possible to recapture this purge stream and combine it with the main flow to the gas scrubber train. In early work the existence of this purge was not recognized, but its effect was corrected for by the counter calibration (see Section 5.4.1).

\section{2 .6 Gas Analyser.}

The inlet and outlet gas streams from the column were analysed using a modified orsat apparatus constructed for the present study. The gas chamber of the Orsat was formed from a $50 \mathrm{ml}$ burette, which enabled the soluble gas to be analysed in concentrations from zero to over $95 \mathrm{v}$. (Gases in concentrations exceeding $50 \mathrm{v} \%$ cannot be analysed in normal orsat equipment.) Mercury was used for the balancing liquid since even liquids such as paraffin and concentrated $\mathrm{NaOH}$ solution were found to absorb significant amounts of ammonia. 


\subsection{Preliminary Procedure}

5.3.1 Rotameter Calibration.

The primary calibrations for the gas rotameters were made by passing the gas from the rotameters through a wet gas meter. For higher flows, two gas meters in parallel were used. An accuracy of $\pm 1 / 2 \%$ at $60 \%$ of design capacity is claimed for the meters. It is therefore expected that calibrations are within $\pm 1 \%$ for low flow rates and $\pm 2 \%$ for higher flow rates where two meters were needed. Comparison of the 3 meters used with each other showed good agreement up to their design capacity. The inert gas meter was calibrated with nitrogen for both glass and stainless steel spherical floats to cover a wide range of flow rates. The "Gilmont" rotameters used for the transferred gas were calibrated with carbon dioxide. Later, a third transferred gas rotameter was added to allow higher flow rates to be measured. This was calibrated using ammonia, by mixing the output with a known nitrogen flow, and analysing the resulting mixture. Secondary calibration for other gases was made by scaling the primary calibration according to the gas properties, using standard rotameter sizing formulae.

The liquid rotameter was calibrated for water and 32 wo $\mathrm{NaOH}$ solution, using a graduated flask and stopwatch. It was not expected that the particle deposition would be a strong function of gas or liquid flow rates, so that high accuracy in determining the absolute flow rates was not essential. Liquid and gas calibration curves are given in Appendix $A$. 
5.3.2 Particle Content of Gases.

The compressed cylinder gases were found to be extremely free of particulate matter. In all cases, when these gases were sampled directly by the counter, the particle concentration was essentially zero. Filtration was therefore not required.

\subsubsection{Column Trials.}

Initial trials with $\mathrm{N}_{2}-\mathrm{CO}_{2}$ mixtures and 32 w\% $\mathrm{NaOH}$ solution gave $\mathrm{CO}_{2}$ absorption well below that indicated by the design calculations. Even with high $\mathrm{CO}_{2}$ inlet concentrations and high liquid flow rates the absorption was inadequate for satisfactory particle removal. This is probably because the predicted enhancement of mass transfer due to chemical reaction far exceeds that which exists in reality. The $\mathrm{CO}_{2}$ was therefore replaced with $\mathrm{NH}_{3}$, and the $\mathrm{NaOH}$ solution with water. Absorption with the new system was quite satisfactory.

\subsubsection{Tests for Liquid Phase Mass Transfer Resistance.}

The traditional test for liquid phase resistance is to vary the liquid fliow rate while keeping a constant inlet gas fiow rate and composition. If the outlet gas composition remains essentially unchanged, then the liquid resistance is negligible. The problem with this test is that the gas side mass transfer coefficient is 
dependent on the gas Reynolds number, measured with respect to the liquid surface rather than to the surface of the dry column. Hence a change in liquid flow rate should produce a small change in outlet gas composition, even if the liquid resistance is negligible.

To test for negligible liquid resistance, a mathematical model was constructed in which the influence of the liquid rate on gas side mass transfer was allowed for. Details of this model are given in Appendix B. Agreement between the model and experimental results was satisfactory, though there was some indication that the liquid phase offered a small mass transfer resistance. Later theoretical work. showed that the surface gas concentrations need not be known in estimating particle deposition rates. Negligible liquid side mass transfer resistance was therefore not neçessary.

\subsection{Operating Procedure}

5.4.1 Counter Calibration for Particle Size.

The primary calibration is made by an internal

adjustment which sets the height of the voltage peak which is produced when a particle of known size is counted. This ensures that the counter distinguishes correctly between particles smaller and larger than $1.2 \mu \mathrm{m}$. D. Since in this work the sizes of the particles counted were always known, accurate primary calibration was not necessary. Nevertheless, the voltage peak heights were occasionally checked and adjusted when required. 
The instrument is designed so that a secondary calibration can be made by feeding an internal reference signal to the photomultiplier tube, and adjusting the output by means of an external control till it matches the internal primary standard. This procedure was followed each time the counter was switched on, and at frequent intervals during operation:

\subsubsection{Counter Calibration for Gas Composition.}

If an inert, aerosol-bearing stream is mixed with varying quantities of the transferred gas and the mixture sampled, the count rate registered per unit volume of inert gas is found to vary according to the composition of the sampled gas. The main reason for this is believed to be that, for gases other than air, all the particles do not pass through the counter sensitive volume. (The manufacturer claims that when air is sampled, all particles do pass through this volume, and hence every particle is counted.) Thus the fraction of gas in which particles are counted will depend slightly on the gas composition. Minor leaks, such as those through the pump seals and in the absorber train, may also be contributing factors.

The counter was therefore calibrated to eliminate this error. A fixed inert particle-bearing stream was mixed with various quantities of transferred gas and sampled with the counter. The sampling scheme was similar to that used during the actual experiment (see Section 5.4.4), but in this case the readings were taken with the transferred gas both present and absent. In 
this way a calibration curve relating the recorded count to the true count for inert gas only was established for various gas compositions. This calibration curve was subsequently used to correct the experimental particle counts for gases of different compositions.

In later runs an improvement in experimental technique was introduced to further reduce calibration errors. In all cases the calibration correction increased with increasing transferred gas concentration. The larger correction was therefore at the base of the column. Usually this correction was less than $10 \%$ of the reading, but in extreme cases it could be up to $40 \%$. It was therefore decided to take the bottom reading with the transferred gas turned off. This eliminated the need for a bottom calibration correction entirely. Also, since the particle losses in the column were negligible in the absence of diffusiophoresis, the "bottom" sample could be withdrawn from the top if desired. The calibration correction for the top reading was normally less than 5\%.

\subsubsection{Preparation for an Experimental Run.}

Some time before the run the liquid storage tank was charged with cold tap water. The water was then heated to about $25^{\circ} \mathrm{C}$ and agitated by means of a circulating pump before being cooled to the operating temperature of $20^{\circ} \mathrm{C}$. This procedure eliminated the formation of liquid droplets in the column, asso- 
ciated with the release of small air bubbles.

To prepare the atomisation solution for the aerosol generator, a small quantity (usually 1 to 10 drops) of the concentrated suspension (10 vo) of particles of the required size, as supplied by Dow, were added to $20 \mathrm{ml}$ of distilled water. The aerosol concentration in the gas is set primarily by choosing the correct dilution of the concentrated suspension, and secondarily by adjusting the gas pressure to the atomising chamber. The lower limit on concentration was set so that the aerosol count would be substantially higher than the background count registered when no aerosol was added to the gas. This background count could arise from droplet entrainment or mist formation in the column, entrained dirt from the gas piping or tubing, dirt in the compressed gases (small), or electronic noise (normally negligible). The background count was usually less than 3,000 particles $/ \mathrm{m}^{3}$, but could exceed 10,000 particles $/ \mathrm{m}^{3}$ for high gas flow rates in some cases. Experimental aerosol concentrations used were typically $\sim 1,000,000$ particles $/ \mathrm{m}^{3}$. Particle-particle interaction in the gas was negligible under these conditions since the average inter-particle distance was $\sim 10^{-2} \mathrm{~m}$ or $\sim 10^{4}$ particle diameters. The volume fraction of particles in the gas phase was $\sim 10^{-12}$. At the start of a run, the counter electronics were warmed up, and a secondary calibration was made for particle size. The counter was then tested by being fed with pure gas, and by being allowed to recycle its own internal gas without any sample being drawn. These tests detected dirt lodged in the sensing 
head, leaking internal filters, high electronic noise levels, and leaks in the counter or scrubber train. These tests were repeated periodically during the run.

The gas flows were set to the required rates. Normally, all of the inert gas was passed through the aerosol generator, and none through the bypass to be mixed with the transferred gas. This eliminated a possible minor error in the transferred gas rotameter reading which could be caused by slight added backpressure from the bypass inert stream. At very high inert flow rates, however, the use of the bypass was necessary. The liquid flow was set at $35 \mathrm{ml} / \mathrm{sec}$ for all runs.

\section{4 .4 Sampling}

The aerosol counter was connected to an inlet (or outlet) sampling point, so that it drew off a continuous stream of gas. Counts were then recorded for a given inert gas volume, measured by the wet gas meter. The gas volume chosen varied from 0.01 to $0.1 \mathrm{ft}^{3}\left(0.00028\right.$ to $\left.0.0028 \mathrm{~m}^{3}\right)$ depending on the inert gas concentration in the gas mixture and the gases being used. Each time the composition of the gas sampled was changed, the counter was left approximately one minute for steady state conditions to be:established. However the count still showed random fluctuations, typically in the range of $\pm 5 \%$. These fluctuations were too large to be caused by the small variations in particle concentration of the solution in the atomiser. It is believed that they were due to very small changes in the 
atomizer gas feed, caused by small pressure fluctuations produced by the aerosol generator gas regulator. The count also occasionally exhibited a slow drift with time.

After the count had stabilized, and provided any drift was minor, four consecutive counts were recorded for calculation purposes (in some cases two counts of longer duration were used). Any counts clearly falling outside the typical range, and that could not be reproduced, were discarded. The sample tube was then connected to the other end of the column and the process repeated to give four more readings. Finally, the sample tube was reconnected to the original end of the column to obtain the four final readings. The aerosol generator was then shut off and the inert gas flow restored to its previous level. The background count rate for the aerosol free gas mixture at the top and bottom of the column was then measured.

The four initial and four final counts were averaged together, and the four central readings were also averaged. From these averages, the appropriate average background counts were subtracted to give the corrected top and bottom counts. These were then used to calculate the particle removal efficiency. By bracketing and averaging the readings in this way the effect of any drift was minimized, and the influence of the random fluctuations was reduced.

Gas analyses were also made at the top and bottom of the column. Two consecutive readings agreeing within $\pm 0.4 \%$ were considered satisfactory. 
5.4.5 Particle Removal in the Absence of Diffusiophoresis.

It was possible that some particle removal was occurring in the column due to mechanisms other than diffusiophoresis. This would probably be primarily the result of inertial deposition, since Brownian diffusion and gravitational settling were expected to be minor for particles in the size range used. Deposition tests were made by passing a high flow of particle-bearing inert gas through the column (inertial deposition is favoured by high velocities), and sampling in the normal manner to detect any change in particle concentration. In no case was any significant particle deposition detected.

\section{4 .6 Isokinetic Sampling.}

It is well known that in sampling particle-bearing streams, error may be introduced if the gas is not sampled isokinetically. In the present experiment, isokinetic sampling was not practicable because of the wide range of gas velocities used in the column, and because the sampling rate was determined by the counter suction rate, which in turn depended on the gas composition. In any case, calculations indicated that the error would be negligible for particles up to $\sim 2 \mu \mathrm{m}$ D. Experiments in which a known flow of inert particle bearing-gas was mixed with a range of known flows of clean inert gas so as to achieve different velocities through the column confirmed this. However, for the larger particle sizes there was some theoretical possibility of significant sampling error. A special sampling head for the 
top of the column was therefore designed, which is shown.mounted on one of the columns in Figure 5.2. In this head, the cross sectional area was gradually increased till the mean gas velocity had been reduced by 36 times. The sample was then drawn from this low velocity region through a tapered offtake. In this way both the velocity of the main gas stream and the velocity of the gas entering the sample tube were kept low enough to make errors due to lack of isokinetic sampling insignificant. It was not possible to easily fit a similar sampling head at the base of the column. The runs made with the special sampling head therefore used the technique where the "bottom" sample is taken from the top of the column, but with the transferred gas turned off.

\subsection{Design of Experiments}

For a given gas mixture, the conditions in the column, and hence the particle removal efficiency, depended on the inlet gas flow rate and composition. (The liquid flow rate was always held constant at $35 \mathrm{ml} / \mathrm{sec})$ These parameters can be completely specified in terms of two independent operating variables, which could be, for example, the flow rates of the two component gases, or alternatively, the inlet flow rate of one gas and the inlet composition of the mixture. Every major series of experiments was made by altering one operating variable while holding the other constant. This procedure had the advantages of producing data whose trends could easily be discerned by appropriate plotting. 
The following types of experiments were performed.

(i) The flow rate of the inert gas was held constant, and the flow rate of the transferred gas to the column was varied.

(ii) The flow rate of the transferred gas to the column was held constant, and the flow rate of the inert gas was varied.

(iii) The inlet composition was held constant, and the total inlet flow rate was varied.

Most experiments were of the first type. However, experiments of all three types were made for the nitrogen-ammonia system with $0.79 \mu \mathrm{m}$ particles, in order to rule out for the whole series of experiments the existence of any unrecognised factors influencing particle removal. 


\section{RESULTS AND DISCUSSION}

\subsection{The Data}

There are 17 sets of experimental data for various gas mixtures and particle sizes. These are given below, listed firstly in order of increasing molecular weight of the inert species, and secondly in order of increasing molecular weight of the transferred species.

\begin{tabular}{|c|c|c|c|}
\hline & Particle & & Particl \\
\hline System & $\frac{\text { size }}{(\mu \mathrm{m})}$ & System & $\frac{\text { size }}{\text { (um) }}$ \\
\hline
\end{tabular}

\begin{tabular}{|c|c|c|c|}
\hline Helium-ammonia & 0.79 & Argon-ammonia & 0.79 \\
\hline Methane-ammonia & 0.79 & $\begin{array}{l}\text { Freon 12-ammonia } \\
\text { Freon } 12 \text {-ammonia }\end{array}$ & $\begin{array}{l}0.79 \\
1.011\end{array}$ \\
\hline $\begin{array}{l}\text { Nitrogen-ammonia } \\
\text { Preliminary data. }\end{array}$ & 0.79 & Freon 12-ammonia & 2.02 \\
\hline $\begin{array}{l}\text { Nitrogen-ammonia } \\
\text { Short column data. }\end{array}$ & 0.79 & $\begin{array}{l}\text { Nitrogen-trimethylamine } \\
\text { Nitrogen-trimethylamine } \\
\text { Nitrogen-trimethylamine } \\
\text { Nitrogen-trime thylamine }\end{array}$ & $\begin{array}{l}0.50 \\
0.79 \\
1.011 \\
2.02\end{array}$ \\
\hline $\begin{array}{l}\text { Nitrogen-ammonia } \\
\text { Nitrogen-ammonia } \\
\text { Nitrogen-ammonia } \\
\text { Nitrogen-ammonia }\end{array}$ & $\begin{array}{l}0.50 \\
0.79 \\
1.011 \\
2.02\end{array}$ & Nitrogen-trimethylamine & 5.7 \\
\hline
\end{tabular}


All data were taken using the normal column $0.77 \mathrm{~m}$ in length, with the exception of the "short column data" for the nitrogen-ammonia system with $0.79 \mu \mathrm{m}$ particles. These data were obtained with the $0.32 \mathrm{~m}$ column.

All experimental results are presented in tabular form in Appendix.D. An explanation of the tables and of the calculation methods used in their preparation is given in Appendix.C. There is one table for each set of data, except in the case of the nitrogen-ammonia mixture with $2.02 \mu \mathrm{m}$ particles, for which there are two tables. These contain data for two series of runs made at different times. The calibration corrections for each of these were found to be different. However, the corrected efficiencies showed excellent agreement, and both sets of data were therefore included.

The results for each gas mixture and particle size are plotted in Figures 6.1 to 6.18 . The preliminary data for the nitrogen-ammonia mixture with $0.79 \mu \mathrm{m}$ particles, which has been presented elsewhere (see Whitmore and Meisen (1973)), is not shown. The remaining results for this mixture with $0.79 \mu \mathrm{m}$ particles are given in three plots (Figures 6.5 to 6.7) corresponding to the three types of experiments made (see section 5.5). Generally all data are shown for each gas mixture, but in some cases runs were made under atypical experimental conditions, and hence could not be plotted. However, these runs were included in the statistical analysis given in section 6.4 .

Three theoretical values for the particle removal efficiency are also shown on each figure. These were calculated 
using the theory developed in Chapter 4, and correspond to the assumptions that the particles travel respectively with either the local gas mean mass velocity, the mean molar velocity, or the velocity predicted by schmitt and Waldmann.

\subsection{General Trends}

Figures 6.1 to 6.18 show that the experimental data tend to lie within the envelope formed by the efficiency predictions of the mean mass and mean molar velocity models. Furthermore, the trend exhibited by the set of data for each specific gas system, particle size, and type of experiment, is very similar to the trends of the three theoretical predictions. Since the experiments cover such a wide range of mass transfer conditions and gas physical properties, such agreement provides strong evidence that the general theory of particle deposition presented in Chapter 4 is correct, at least as a first order approximation. This is not surprising considering the broad generality of that theory.

At the same time, the position of the data relative to the three models varies with both gas system and particle size, and the data as a whole do not correlate well around the predictions of any specific model. As discussed in section 6.3, in almost every case the possible systematic errors alone are large enough to account for the lack of correlation with the mean mass velocity. model. This does not mean, however, that the position of the 
data relative to the various models is without significance. The error estimates are the maximum expected errors, and not the probable errors, which are much smaller. In any case, it is unlikely that the variation in data position is caused primarily by systematic errors, as these would tend to nullify the observed similarity between the trends shown by each set of data and the corresponding model predictions.

The only obvious remaining explanation for the experimental results obtained is that the particles were exhibiting transition regime behaviour. : According to the theory developed in Chapter 3, large particles will move with the mean mass velocity of the fluid. Small particles will travel atessentially the Schmitt and Waldmann velocity, if their interactions with both molecular species are similar, as discussed in Chapter 2. With dissimilar interactions, the particles will move at a slightly different velocity, which intuitively can be expected to lie between the mean mass and mean molar velocities. Hence the removal efficiencies in the transition regime should fall between the limits prescribed by the predictions of the mean mass and mean molar velocity models. Furthermore, for a given gas system and particle size, these efficiencies should show the same trend as the model predictions, when experimental conditions are systematically varied. ${ }^{\dagger}$ Since the outlined behaviour is, identical to that exhibited by the experimental results obtained in

$T_{A}$ fuller discussion of the theory of particle removal in the transition regime will be found in Appendix $\mathrm{E}$. 
the present study, it is concluded that the particles generally fell within the transition regime.

The transition regime behaviour precludes definite confirmation that large particles move at the mean mass velocity. There is, however, an indication that they do, since for small Knudsen numbers the data tend to correlatelaround the corresponding model predictions. In contrast, only a small fraction of the data agree with the predictions of the mean molar velocity model. This suggests that the theories of Derjaguin et al. (1966) and Derjaguin and Yalamov (1972), which predicted this velocity for large particles, may be incorrect.

\subsection{Accuracy and Errors}

\section{3 .1 General}

The errors can be divided into two groups. Random errors produce scatter in the data, but an increasingly accurate mean value can be obtained as more readings are taken. Systematic errors lead to an offset between the mean experimental value and the true value. These two types of errors are discussed in the following two subsections. Sample error calculations appear in Appendix $C$.

An important test of the experimental procedure is the reproducibility of the data. Good reproducibility was obtained within the normal experimental scatter for consecutive readings taken under the same experimental conditions." (Readings of this type bear the same run number in Appendix D.) 
In addition, replicate runs, sometimes made weeks or months later, showed good agreement with the original data.

\subsubsection{Random Errors}

There are two main sources of random errors. The most important is the random change in the aerosol concentration produced by the aerosol generator. The second is the error in determining the time for a given volume of gas to pass through the gas meter. This is estimated to introduce an error of approximately $\pm 1 \%$ in the particle count. Both these errors were reduced by the bracketing and averaging methods used in the data analysis (see Appendix $c$ ), and their influence on the estimated efficiencies is best obtained from statistical analysis of the final data (see Section 6.4).

\subsubsection{Systematic Errors}

Several sources which might give rise to significant errors were recognized, and these are discussed in approximate order of decreasing importance.

The main error arises in the aerosol counter calibration for gas composition. The calibration effect is usually smal1, producing a change in the true count of less than $10 \%$. However, the experimental scatter in determining the calibration curve is appreciable in relation to this correction, being of the order of $\pm 5 \%$. Also, the calibration correction becomes less definite as the mole fraction of the transferred gas increases, 
because of increased random errors.

Errors in gas analyses do not influence the experimental removal efficiency, but they do affect the theoretical model predictions. Since the gas analyses for each experimental condition were normally done only twice to obtain readings within $\pm 0.4 \%$, there is no estimate of the scatter and the error is treated as systematic.

Another source of error in the gas analysis is the presence of more than two components in the gas mixture. Since the aerosol was generated from a water suspension, the gas mixture contained some water vapour. However, calculations showed that this quantity was negligible. A second possible source of water vapour was evaporation of the liquid film in the column. This was also assessed to be negligible, since no. water vapour could be detected in the gas leaving the column. In any case, the saturation concentration of water vapour at $20^{\circ} \mathrm{C}$ (the approximate temperature of the exiting gas) is quite small. Although release of heat of absorption at the water surface would enhance evaporation, the transfer of this vapour into the bulk of the gas is in turn inhibited by the strong flux of transferred gas towards the liquid surface. (Errors in the rotameter readings do not directly introduce any errors in estimating the theoretical efficiencies, since these are based on the gas analyses and component molecular weights alone.) Other gas components can also result from impurities in the cylinder gases (see Table II). This was estimated to introduce an absolute error in the fractional efficiencies 
predicted by the models of order $(x+y) / 8$, where $x$ and $y$ are the fractional impurity levels in the inert and transferred gas components (see Appendix C). Since most gases were relatively pure, this source of error was small.

Another source of error arises from particle losses due to inertial deposition in the column. The existence of these losses was however never detected, and theoretical calculation indicated that they would be negligible.

Lack of isokinetic sampling could introduce error, but this could not be detected in experimental tests. Moreover, a specialsampling port (see Chapter 5) was used for the larger particles, so that any error was minimized.

Although the column was designed to operate at close to isothermal conditions, the heat liberated during the gas absorption inevitably produced a temperature rise in the liquid as it passed through the column. This was typically a few degrees Celsius, but could be as high as $10^{\circ} \mathrm{C}$ under extreme conditions. However, the overall change in gas temperature was substantially less than this, since, although the gas may be heated by the liquid at the base of the column, it loses heat back to the liquid at the top of the column. The overall change in gas temperature was therefore small. For example, under the highest mass transfer rates used with the nitrogen-ammonia system, the liquid temperature increased $10^{\circ} \mathrm{C}$, while the gas temperature increased only $3^{\circ} \mathrm{C}$ in passing through the column.

On this basis, the inhibition of particle deposition caused by thermophoresis was estimated to be negligible (see 
Whitmore and Meisen, to be published, Canadian Journal of Chemical Engineering). The above argument presupposes that the thermophoretic force is at no point strong enough to completely suppress particle deposition, a supposition which was confirmed by calculation.

The last source of error was particle generation in the column. Particles could be formed by entrainment of liquid droplets or dirt from interior surfaces. However, tests showed that these sources were not significant. In any case they would be included in the background count, and hence eliminated (see Section 5.4).

The other source of particles was from mist formation in the column. Although the outlet water vapour concentration was normally small, it is still conceivable that the gas could become locally supersaturated. This would occur because the gas in contact with the liquid at the base of the column picks up some water vapour. It then tends to move into the cooler regions further up the column. More importantly, the gas volume shrinks as the transferred gas is absorbed, which in turn concentrates the water vapour. These considerations are probably not important except under the extreme mass transfer conditions encountered in some of the freon 12-ammonia experiments. For example, in one instance the ammonia transfer was so large that the volumetric gas flow decreased by a factor of 6 between the column inlet and outlet.

The difficulty in assessing this error is that the droplet formation usually only occurred in the presence of the 
particles, which presumably acted as the initial condensation nuclei for the supersaturated gas. Thus it was not possible to count the number of mist particles, or test for their presence with the flow of aerosol particles shut off. However, it was found that spurious results were obtained whenever unusually large numbers of particles were recorded on the counter channel on which the latex particles were not registering. Normally the count on that channel would be small. Fortunately, the phenomenon of mist formation as judged by this test could often be avoided by using a startup procedure which minimized high transient gas temperatures. Also, mist formation was typically not established for some time, if at all. However, since no direct test could be made, the presence of small but significant numbers of mist droplets in the same size range as the latex particles cannot be altogether ruled out. The error is expected to be small, and to be present only when the fractional volume change for the gas passing through the column is large. Table IV lists for each gas system and particle size, estimates of the possible systematic errors in the predictions of the mean mass velocity model and in the measured experimental efficiency, as well as the sum of these errors. The model error is a combination of inaccuracies resulting from the gas analysis and the presence of more than two gas components in the gas mixture. The experimental error quoted arises from uncertainties in the counter calibration, since the other experimental errors were all estimated to be negligible. 
Table IV

Estimates of Systematic Errors

\begin{tabular}{|c|c|c|c|c|}
\hline $\begin{array}{c}\text { Gas } \\
\text { mixture }\end{array}$ & $\begin{array}{l}\text { Particle } \\
\text { size }(\mu \mathrm{m})\end{array}$ & $\begin{array}{l}\text { Possible } \\
\text { absolute } \\
\text { error in } \\
\text { theoretical } \\
\text { model pre- } \\
\text { diction }\end{array}$ & $\begin{array}{l}\text { Possible } \\
\text { absolute } \\
\text { error in } \\
\text { experimental } \\
\text { efficiency }\end{array}$ & $\begin{array}{l}\text { Total } \\
\text { possible } \\
\text { absolute } \\
\text { error }\end{array}$ \\
\hline $\mathrm{He}-\mathrm{NH}_{3}$ & 0.79 & \pm .01 & \pm .04 & \pm .05 \\
\hline $\begin{array}{l}\mathrm{N}_{2}-\mathrm{NH}_{3} \\
\text { Preliminary } \\
\text { data }\end{array}$ & 0.79 & .01 & .06 & .07 \\
\hline $\begin{array}{l}\mathrm{N}_{2}-\mathrm{NH}_{3} \\
\text { Short } \\
\text { column data }\end{array}$ & 0.79 & .01 & .05 & .06 \\
\hline $\mathrm{A}_{r}-\mathrm{NH}_{3}$ & 0.79 & .01 & .05 & .06 \\
\hline
\end{tabular}


Table IV/cont'd ....

\begin{tabular}{|c|c|c|c|c|}
\hline $\begin{array}{l}\text { Gas } \\
\text { mixture }\end{array}$ & $\begin{array}{l}\text { Particle } \\
\text { size }(\mu \mathrm{m})\end{array}$ & $\begin{array}{l}\text { Possible } \\
\text { absolute } \\
\text { error in } \\
\text { theoretical } \\
\text { model pre- } \\
\text { diction }\end{array}$ & $\begin{array}{l}\text { Possible } \\
\text { absolute } \\
\text { error in } \\
\text { experimental } \\
\text { efficiency }\end{array}$ & $\begin{array}{l}\text { Total } \\
\text { possible } \\
\text { absolute } \\
\text { error }\end{array}$ \\
\hline Freon $12-\mathrm{NH}_{3}$ & 0.79 & .02 & .08 & .10 \\
\hline Freon $12-\mathrm{NH}_{3}$ & 1.011 & .02 & .08 & .10 \\
\hline Freon $12-\mathrm{NH}_{3}$ & 2.02 & .02 & .08 & .10 \\
\hline $\mathrm{N}_{2}-\mathrm{N}\left(\mathrm{CH}_{3}\right)_{3}$ & 0.50 & .01 & .03 & .04 \\
\hline $\mathrm{N}_{2}-\mathrm{N}\left(\mathrm{CH}_{3}\right)_{3}$ & 0.79 & .01 & .04 & .05 \\
\hline $\mathrm{N}_{2}-\mathrm{N}\left(\mathrm{CH}_{3}\right)_{3}$ & 1.011 & .01 & .05 & .06 \\
\hline $\mathrm{N}_{2}-\mathrm{N}\left(\mathrm{CH}_{3}\right)_{3}$ & 2.02 & .01 & .06 & .07 \\
\hline $\mathrm{N}_{2}-\mathrm{N}\left(\mathrm{CH}_{3}\right)_{3}$ & 5.7 & .01 & .08 & .09 \\
\hline
\end{tabular}




\subsection{Statistical Analyses}

The data for each gas mixture and particle size were statistically analysed. In addition, the extensive data for the nitrogen-ammonia system with $0.79 \mu \mathrm{m}$ particles were also divided into subsets containing the results of the three types of experiments performed (see Section 5.5). These subsets were analysed separately. The aim of the statistical analysis was to confirm the general conclusions already reached in section 6.2. It was also desired to test for possible influences of transition regime behaviour and inertial deposition losses, which were not obvious from a visual inspection of Figures 6.1 to 6.18 . Details of the statistical methods used are given in Appendix $\mathrm{E}$.

In the first part of the analysis a mean offset was calculated from the difference between each experimental efficiency and the corresponding model prediction. The calculation was performed for each individual set and subset of data, using each of the three theoretical models. This establishes the relative position of the data with respect to the models, the model that gives the best agreement, and the significance of the offset from the mean mass velocity model in terms of the scatter in the data.

The results of this analysis are given in Table $\mathrm{V}$. The offset from the mean mass velocity model, given in column 4, is positive when the experimental efficiencies are, on average, greater than the model predictions. The significance of this offset, determined at the 90 and $95 \%$ confidence levels using the statistical t-test, is shown in column 5. Offsets of lower significance are classified as not significant (N.S.). A signi- 
Table V

Comparison of Data with Theoretical Models

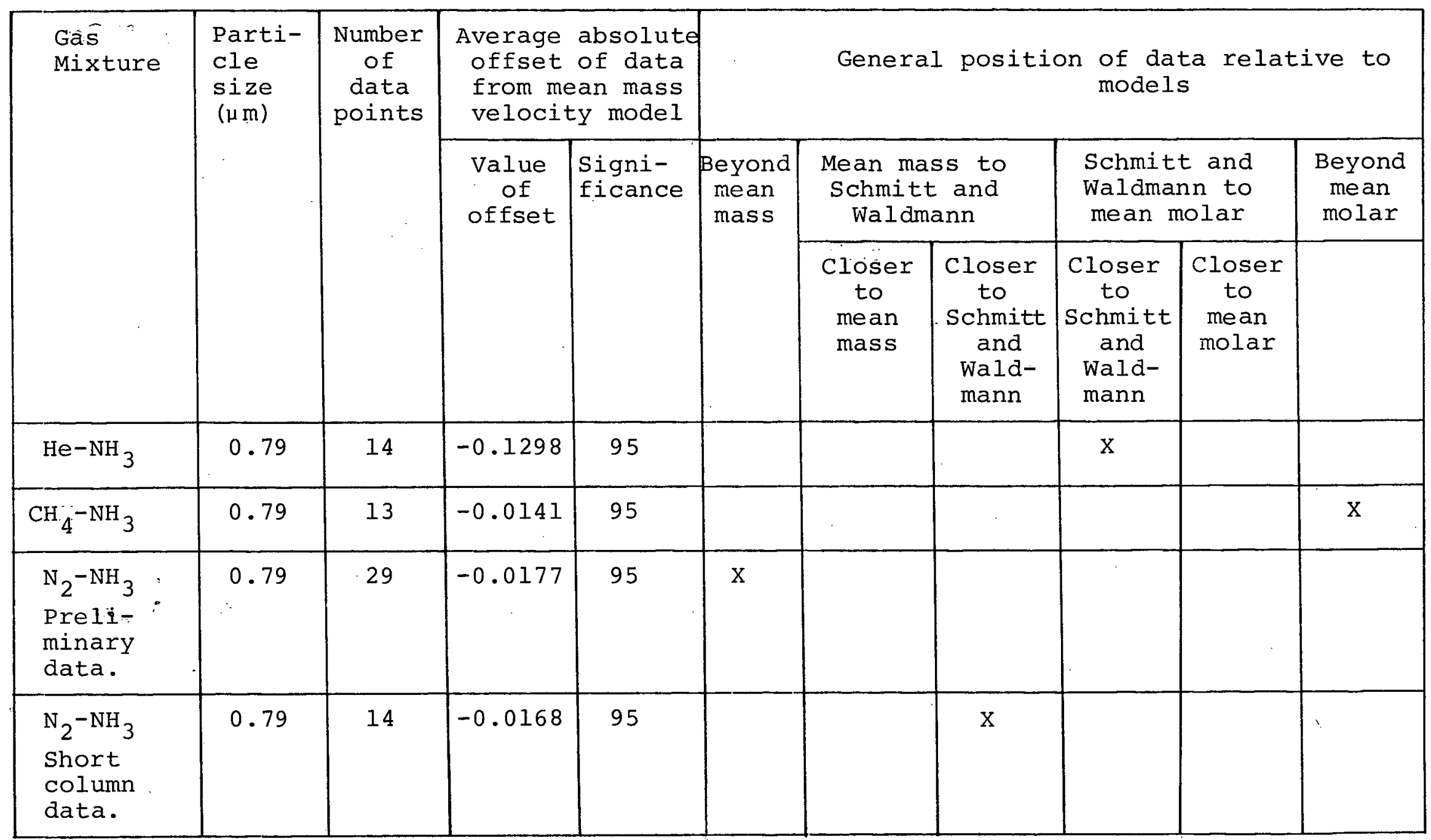


Table V/cont'd ...

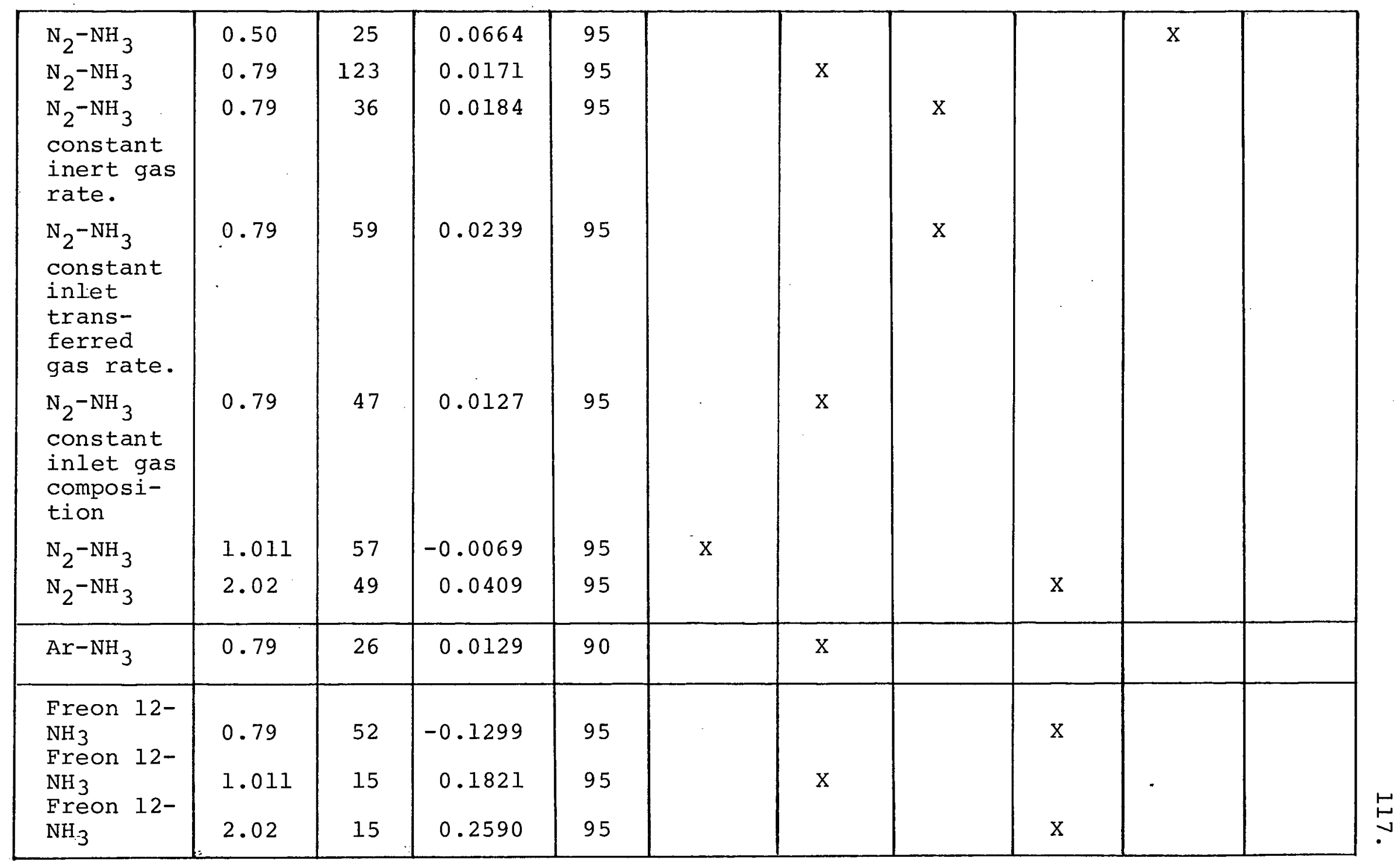


ficant offset means that, considering only the random error as evidenced by the scatter in the data, the experimental results are probably different from the theoretical model predictions. The final six columns indicate the position of the data with respect to the predictions of the three models, and show which model gives the best agreement. Thus, in the case of the first entry, which is for helium-ammonia with $0.79 \mu \mathrm{m}$ particles, the data lie between the predictions of the Schmitt and Waldmann model and the mean molar velocity model, and are closer to the former. Classification in this manner is possible because the predictions of the Schmitt and Waldmann model always fall between those of the other two.

Based on the mean offset of the data from the predictions of each model, only the results for methane-ammonia with $0.79 \mu \mathrm{m}$ particles lie outside the theoretical envelope and beyond the efficiencies predicted by the mean molar velocity model. However this offset is not significant even in terms of the scatter in the data. In fact the three model predictions are so close together for this gas mixture that the data show almost as good agreement with the mean mass velocity model as with the others. At the other extreme, only the preliminary data for nitrogen-ammonia with $0.79 \mu \mathrm{m}$ particles, and the data for nitrogen-ammonia with $1.011 \mu \mathrm{m}$ particles, lie beyond the mean mass velocity model. Although in each case the offset is significant in terms of the scatter in the data, it is small in comparison with the possible absolute errors. The experiments are therefore in agreement with the tentative conclusion reached 
in Chapter 4, namely that the fluid mean mass and mean molar velocities are the limiting velocities that can be attained by a particle in any regime.

For the 17 combinations of gas mixtures and particle sizes studied, 6 give best agreement with the mean mass velocity theory, 8 with that of Schmitt and Waldmann, and only 3 with the mean molar velocity theory. Of the latter, the methane-ammonia case is certainly not significant, as stated before. There is no obvious reason to discount the significance of the other two cases. However, the weight of evidence casts serious doubt on the validity of the mean molar velocity theory for large particles. This is not surprising if one accepts the criticisms of this theory made in chapter 2.

The second part of the analysis involved testing whether transition behaviour or inertial deposition were having a significant influence on the experimental results. All the data sets and subsets used previously were tested. In addition, where more than one particle size had been experimentally examined with a given gas mixture, all the data for the mixture were grouped together. (The data for $5.7 \mu \mathrm{m}$ particles were not included in the nitrogen-trimethylamine group because of their large scatter.) This had several potential benefits. The ranges of values for the parameters used in testing for the two effects were greatly increased, and more data points were available than for individual particle sizes. Thus the chances of obtaining significant results were improved. Also, systematic errors were expected to have dissimilar effects on the results for particles of different sizes. 
It was therefore hoped that by grouping these results, systematic errors, which could mask trends within individual data sets, would behave as random errors.

The existence of transition regime behaviour can be investigated using the Knudsen number. The parameter chosen in testing for inertial deposition was the Reynolds number multiplied by the particle diameter squared. This parameter is closely related to the stokes stopping distance. For first-order testing over the limited Reynolds number and particle diameter ranges covered in this work, it should be adequate to assume that inertial deposition exhibits a linear dependence on the stopping distance parameter.

As shown in Table $V$, the value of the average offset from the mean mass velocity model varied with gas mixture and particle size used. This indicates that the particles are probably in the transition regime, but is not conclusive. For example, it does not confirm that the trend shown by the data is generally similar to that of the model predictions. In fact, no simple and unambiguous test for transition regime behaviour could be devised.

A transition regime model for particle removal efficiency was therefore constructed, and the data were tested to see whether they could be adequately described by it. The detailed development of the model is given in Appendix E. It was derived by assuming that particle velocities in the transition regime could be represented as the sum of appropriate small and large particle velocity components. One component was obtained by 
multiplying the small particle velocity (i.e., Schmitt and Waldmann's result) by a linear function of Knudsen number, and the other was obtained by multiplying the large particle velocity (i.e., the mean mass velocity of the fluid) by one minus this function. The assumption of linearity should be adequate over the Knudsen number range involved.

The model yielded the following equation for the removal efficiency due to diffusiophoresis and inertial deposition in the transition regime:

$$
\varepsilon_{p}=A_{r}\left(\varepsilon_{S W}-\varepsilon_{M A}\right)+B_{r}\left(\varepsilon_{S W}-\varepsilon_{M A}\right) K n+C_{r} S+\varepsilon_{M A}
$$

Here $S$ is the stopping distance parameter, and $\varepsilon_{M A}$ and $\varepsilon_{S W}$ are the predictions of the mean mass and schmitt and Waldmann velocity models, respectively. The magnitude of the coefficients $A_{r}$ and $\mathrm{B}_{r}$ depend on the quantitative relationship between particle behaviour and Knudsen number in the transition regime, while $\mathrm{C}_{\mathrm{r}}$ depends on the rate of inertial deposition.

The existence of the two effects may be confirmed if the experimental values for removal efficiency in a data set can be satisfactorily correlated using the equation. The tests were made using a stepwise multiple regression technique, as described in Appendix E.

The results of the tests for transition behaviour were inconclusive, even for the grouped data. This was attributed to the influences of random scatter in the data and of systematic errors. It does not imply, however, that the effect did not 
exist, but only that the data were not accurate enough to establish with confidence its presence. For the same reasons, the influence of inertial deposition was also unclear, but it can be concluded that it had a very small influence, being responsible for a loss of much less than $1 \%$ for $1 \mu \mathrm{m}$ particles under typical conditions.

\subsection{Detailed Analysis}

\section{5 .1 Helium-Ammonia}

The results for $0.79 \mu \mathrm{m}$ particles (Figure 6.1 ) show moderate agreement with Schmitt and Waldmann's theory. The mean Knudsen number for the data was 0.24 , which is considerably higher than for any other system, and reflects the large mean free path of helium molecules. It is therefore very probable that the particle behaviour fell well into the transition regime. The wide range of molecular weights allowed good resolution between the models,but at the same time tended to give a larger counter calibration error.

For most gas mixtures the flow rate of the inert gas was set to give turbulence with respect to the dry column, and the flow rate of the transferred gas was varied. However, for helium this was economically prohibitive, so instead the ammonia flow rate was held constant and the helium flow rate varied. The result was that the outlet Reynolds number fell below 2000 in most runs, and in the worst case went as low as 320. While this was undesirable in a study specifically aimed at turbulent 
conditions, the results indicate that the transition flow is not influencing the trend of data. This was predicted by the general theory derived in Chapter 4, which showed that particle removal is independent of flow conditions.

\subsubsection{Methane-Ammonia}

The significant feature of this mixture is the very small ratio of molecular weights, 16.04/17.03. This means resolution between the models is poor. At the same time no calibration correction was required, and the calibration error was estimated to be small. The excellent agreement between the data and the models (see Figure 6.2) indicates that the experimental technique was satisfactory, and that the general theory given in Chapter 4 is substantially correct in the special case of similar molecular weights.

\subsubsection{Nitrogen-Ammonia, Preliminary Data}

These results for $0.79 \mu \mathrm{m}$ particles were obtained very early during the experimental work, after which time several improvements were made in the equipment and technique. Although there is a small offset between these and later data, it is not significant in terms of the possible experimental errors. 


\subsubsection{Nitrogen-Ammonia, Short Column Data}

Since the experimental apparatus was constructed before the theory was derived, a short column was included so that end effects could be subtracted out if necessary. The theory indicated, however, that end effects were not relevant, and an exploratory set of data taken using $0.79 \mu \mathrm{m}$ particles in the short column confirmed this after comparison with the regular column data (see Figure 6.3).

\section{5 .5 Nitrogen-Ammonia}

There was a tendency for the data to move down towards the mean mass velocity model prediction as the particle size increased from 0.5 to $1.011 \mu \mathrm{m}$, as shown in figures 6.4 to 6.9 . (The $2.02 \mu \mathrm{m}$ particles show a reverse in this trend, which is, however,small in relation to possible errors.) This provides good evidence that the particles fell into the transition regime, even though statistical tests were inconclusive.

The most comprehensive series of runs in the whole study was made with this gas mixture, using $0.79 \mu \mathrm{m}$ particles. The three different sets of data taken with constant flow rate to the column of the inert gas, of the transferred gas, and with constant inlet gas composition (Figures 6.5 to 6.7 ) enabled a wide range of experimental conditions to be investigated. They also minimized the possibility of important variables or effects being overlooked because of their strong correlation with other parameters. Individual analysis for the three sets of data showed 
that they were statistically indistinguishable from each other in both their position and trend with respect to the mean mass velocity model.

\section{5 .6 Argon-Ammonia}

The behaviour of this gas mixture with $0.79 \mu \mathrm{m}$ particles was expcted to be very similar to that of nitrogen-ammonia, since the physical properties of the two are so similar. This was confirmed by experiment (see Figure 6.10).

\subsubsection{Freon 12-Ammonia}

The data for this gas mixture are difficult to explain (Figures 6.11 to 6.13). Although the possible experimental error is quite large, it is still surprising that the data tend to agree with Schmitt and Waldmann's theory for all particle sizes, since the Knudsen numbers are comparatively small. For example, the mean Knudsen number for the $1.011 \mu \mathrm{m}$ particle data is 0.04 . This does not, however, disprove the contention that the mean mass velocity model is appropriate for large particles, since there is significant possible error, and since transition behaviour may stili persist.

The freon 12 molecule is relatively large and complex compared to that of ammonia. It is therefore possible that its interaction with the particle surface is somewhat different. This could lead to more persistent transition regime behaviour 
than that shown by mixtures of similar molecules. 'In particular, the onset of transition might be more rapid with increasing Knudsen number.

The experimental problems encountered with the freon 12ammonia system were more severe than in any other case. Because of the high molecular weight of freon 12, very large quantities of ammonia had to be transferred to obtain satisfactory particle removal. This created the potential problem of mist formation outlined in section 6.3. Also the gas analyses at the top of the column gave very high freon 12 concentrations, which were difficult to analyse accurately. Furthermore, the particle counter was found to become more unreliable as the ratio of molecular weights of the component gases increased. The ratio of 120.93/ 17.03 for this system was the largest encountered. There is therefore a possibility that the errors shown in Table IV may be an under-estimate in the case of this mixture.

\subsubsection{Nitrogen-Trimethylamine}

This gas mixture was chosen to test the theory for a transferred gas other than ammonia. The high molecular weight for trimethylamine of 59.11 was also useful in improving the resolution between the models. The data show no obvious trend with particle size. (see Figures 6.14 to 6.18). The runs for $5.7 \mu \mathrm{m}$ particles were stretching the capabilities of the experimental system, and the scatter with them is severe. According to the statistical analysis given in Table V., the offset of $5.7 \mu \mathrm{m}$ data from the mean mass velocity model is very small, and statis- 
tically not significant. However, although the Knudsen number is small, considering the scatter and the limited number of data points, this cannot be interpreted as a validation of the mean mass velocity theory.

\subsection{Further Discussion}

It is reasonable to conclude that the general particle deposition model presented in Chapter 4 is indeed satisfactory, and furthermore, that inertial deposition was a small or insignificant effect in the present experimental study.

It seems very probable that the tendency of data to be offset from the mean mass velocity model is due at least in part to transition behaviour. However, definitive proof of this hypothesis using statistical methods was not possible. This was presumed to be primarily due to the confounding influence of small systematic experimental errors in the data.

The experiments of other workers may elucidate the situation by indicating the extent of the transition regime, and thus showing whether transition behaviour should be expected. Storozhilova (1964) was able to examine particle behaviour over a continuous range of knudsen numbers by altering the gas pressure in the experimental unit. The data appear to show a rapid transition from small particle behaviour to large particle behaviour over a Knudsen number range of approximately 1 to 0.5 . There are three difficulties in accepting this conclusion. Intuitively it seems unlikely that continuum conditions could be 
established for a Knudsen number of 0.5 . Also the lowest Knudsen number for which data are presented is approximately 0.3 , so the true establishment of large particle behaviour is still in doubt. Lastly, the data in the Knudsen number range of 0.3 to 0.5 are shown to correlate with the fluid mean molar velocity. However, the theory predicting this velocity was substantially criticised in Chapter 2 , and it is not in agreement with the present experimental results. For these reasons storozhilova's data cannot be regarded as determining the extent of the transition regime.

The earlier experimental work of Schmitt and Waldmann (1960) was critically reviewed in Chapter 2. However, while their measurements of absolute particle velocities may not be reliable, the data, which cover a continuous knudsen number range, could indicate the extent of the transition regime. The authors have fitted curves to this data which imply that large particle behaviour is established at a Knudsen number of approximately 0.2. However, in many cases the data diverge from these curves as the Knudsen number falls to 0.1 , which was the limit of their experiments. The limiting Knudsen number for the transition regime is therefore again unclear, but is probably lower than 0.2 , a figure that seems more realistic than that suggested by Storozhilova's data.

Data on thermophoresis are available that cover a much wider Knudsen number range. For example Waldmann and Schmitt (1966) quote experiments with Knudsen numbers ranging from 0.05 to 5. Since diffusio- and thermophoresis are related phenomena, the transition region might be expected to cover a similar range 
for both cases. The data show that transition starts at a Knudsen number of about 5 , extends below 0.1 , and may not be complete even at 0.05 . This would imply that almost all the data in this work should show some transition behaviour.

In the case of thermophoresis the transition between regimes can be described by a smooth curve. Such simple behaviour may not be exhibited in diffusiophoretic systems. Although the procedure used to calculate the mean free path in this work was adequate for the purpose, it is somewhat simplistic. In fact each molecular species has its own mean free path which is a function of the mole fractions, molecular weights, and collision diameters of both molecular species (see for example Chapman and Cowling (1964)). It therefore is plausible that the onset of transition for the different gas species can occur at different conditions. If this is so, the particle behaviour in the transition regime may be quite complex, and not representable by such a simple curve as in the case of thermophoresis.

A further factor which may complicate transition behaviour is the different interaction characteristics of the molecular species with the particle surface, discussed in Section 6.5. The investigation of these possibilities fell outside the scope of present experiments. However, they may have been additional factors in confusing the search for definite proof of transition behaviour. It is also possible that they have mislead other workers as to the extent of the transition regime.

It is concluded that in most of the runs it is highly 
probable that the particles exhibited some transition behaviour, and that this factor is in part responsible for the variation in data position for different systems and particle sizes. In addition, there is some indication that large particles do travel at, or close to the local mean mass velocity of the fluid, as predicted in Chapter 3. The data certainly do not support the contention that these particles travel at the mean molar velocity. 
131 .

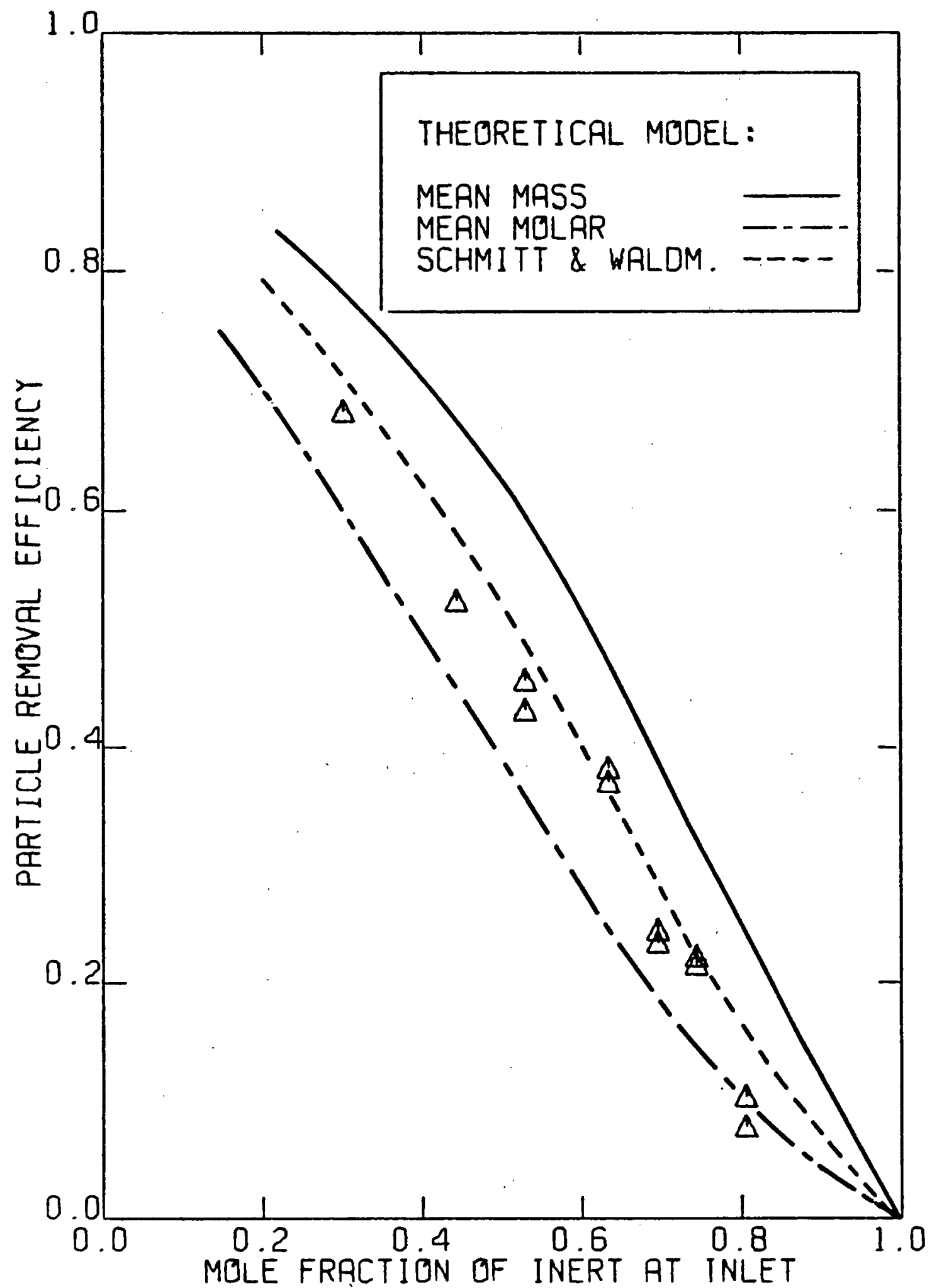

Figure 6.1 Results for helium, ammonia, 0.79 micron diameter particles. Inlet flow rate of transferred gas held constant, at $6.0 \times 10^{-4}$ $\mathrm{m}^{3} / \mathrm{sec}$. 


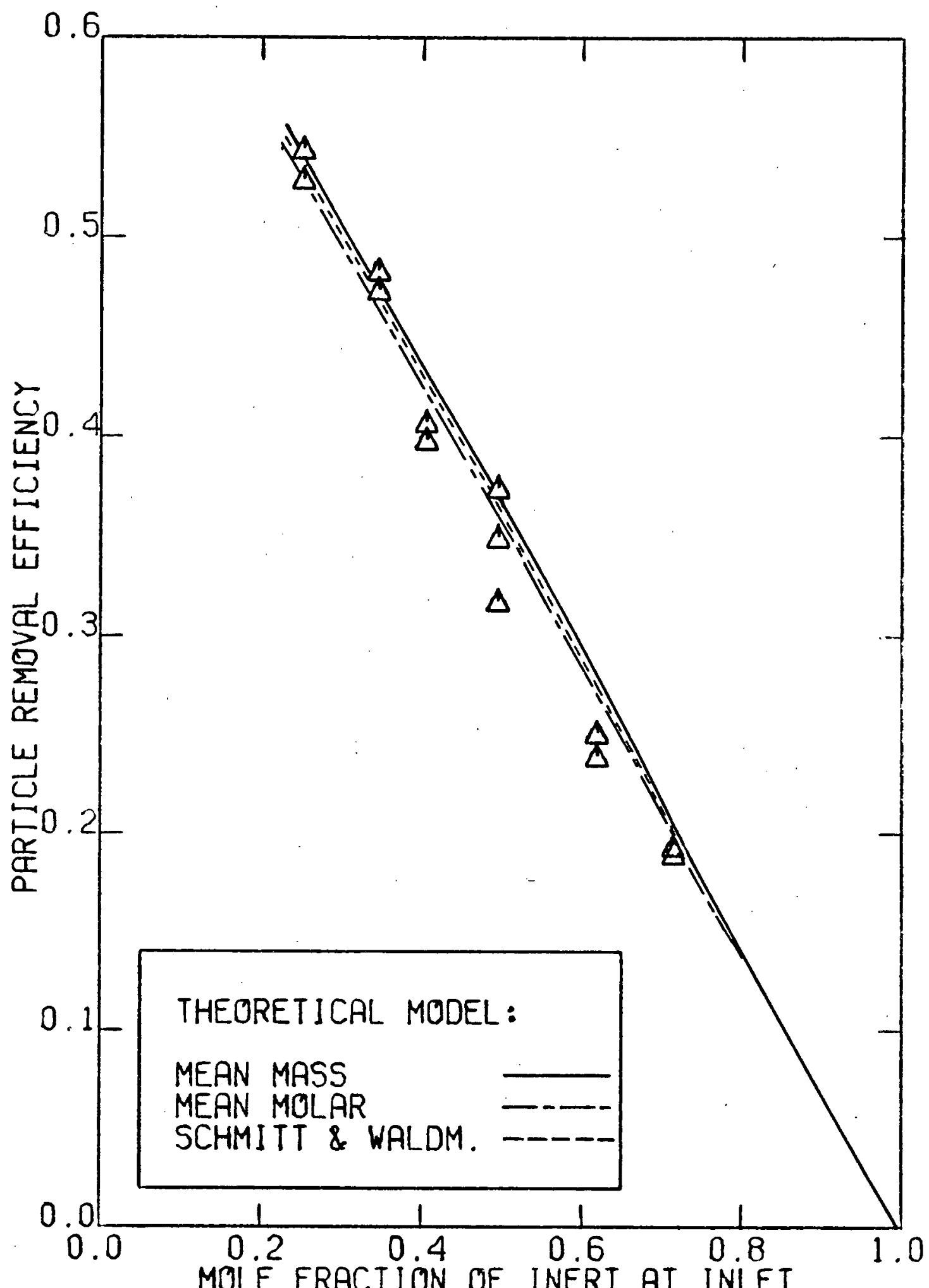

Figure 6.2 Results for methane, ammonia, 0.79 micron diameter particles. Flow rate of inert gas held constant at $5.98 \times 10^{-4} \mathrm{~m}^{3} / \mathrm{sec}$. 


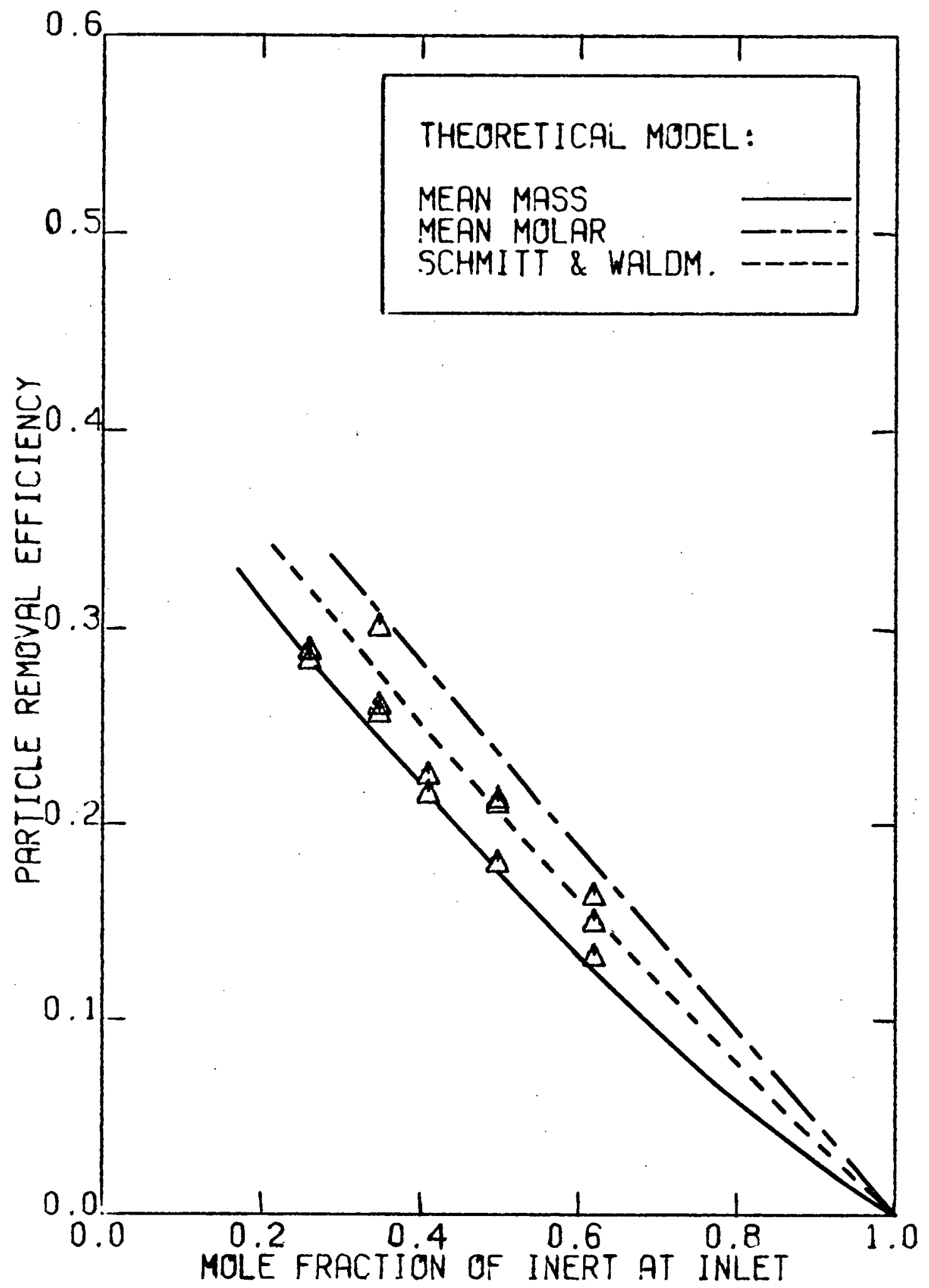

Figure 6.3 Results for nitrogen, ammonia, 0.79 micron diameter particles - short column. Flow rate of inert gas held constant at $6.0 \times 10^{-4} \mathrm{~m}^{3} / \mathrm{sec}$. 


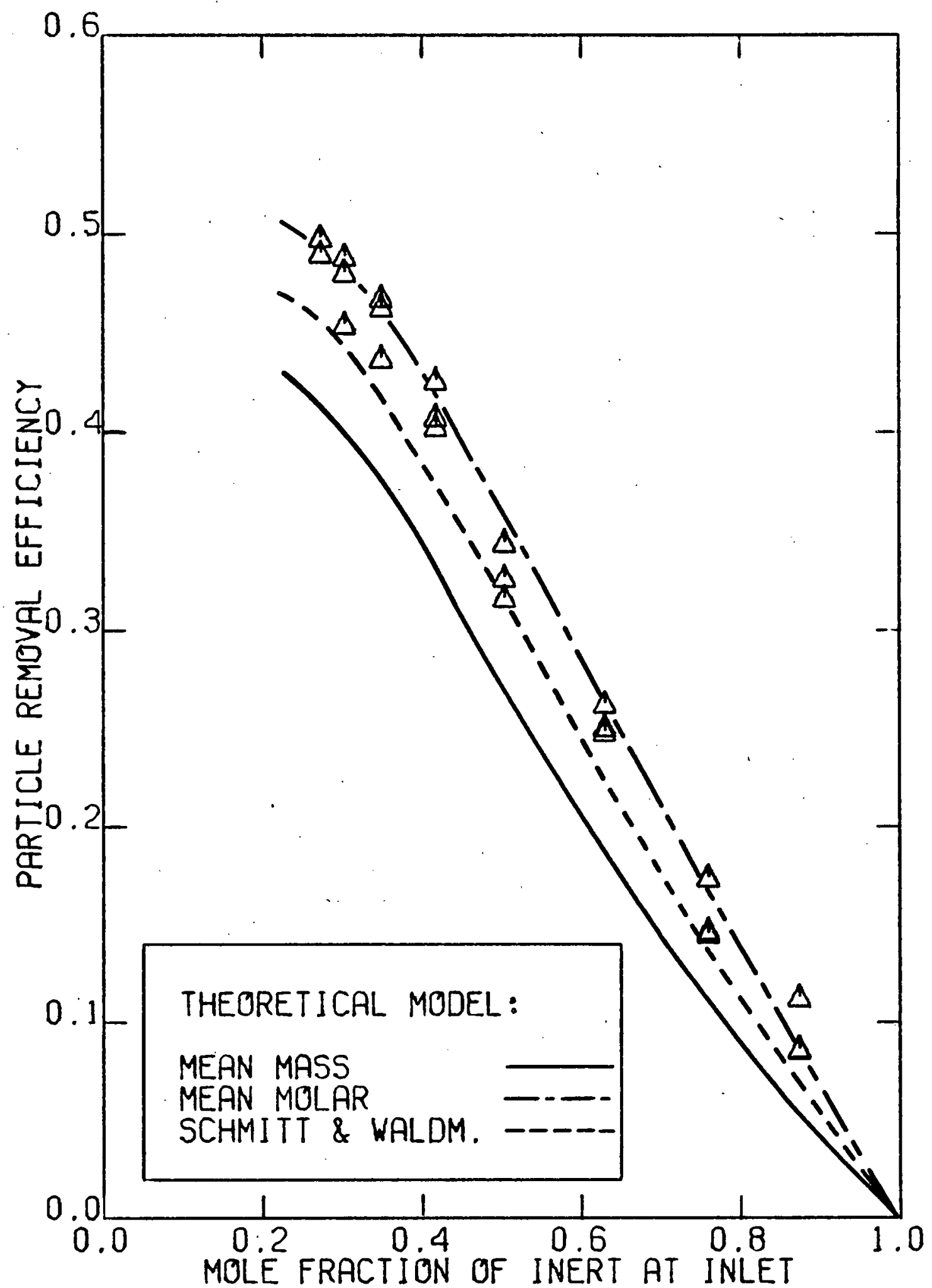

Figure 6.4 Results for nitrogen, ammonia, 0.50 micron diameter particles. Flow rate of inert gas held constant at $6.0 \times 10^{-4} \mathrm{~m}^{3} / \mathrm{sec}$. 


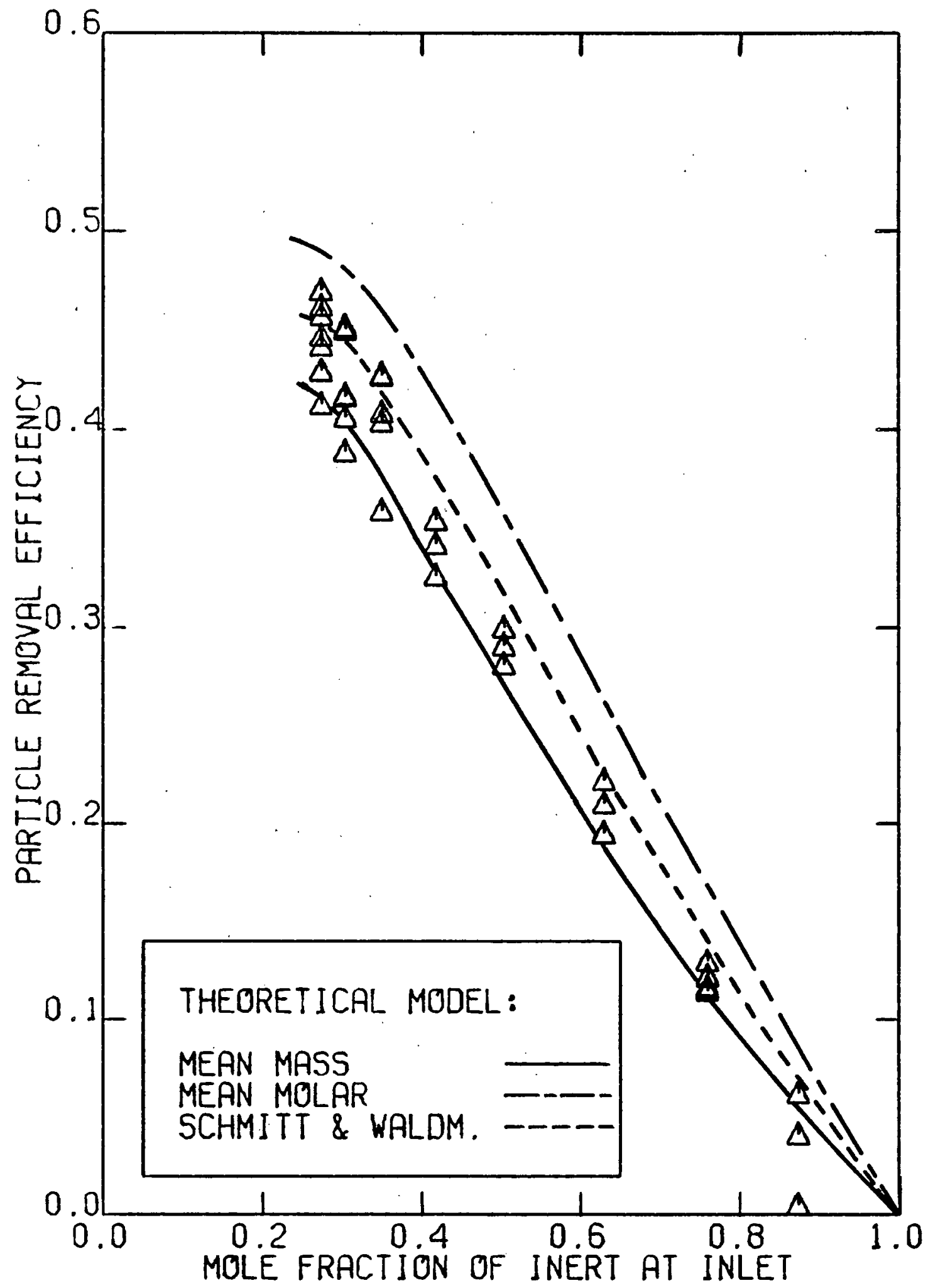

Figure 6.5 Results for nitrogen, ammonia, 0.79 micron diameter particles. Flow rate of inert gas held constant: at $6.0 \times 10^{-4} \mathrm{~m}^{3} / \mathrm{sec}$. 


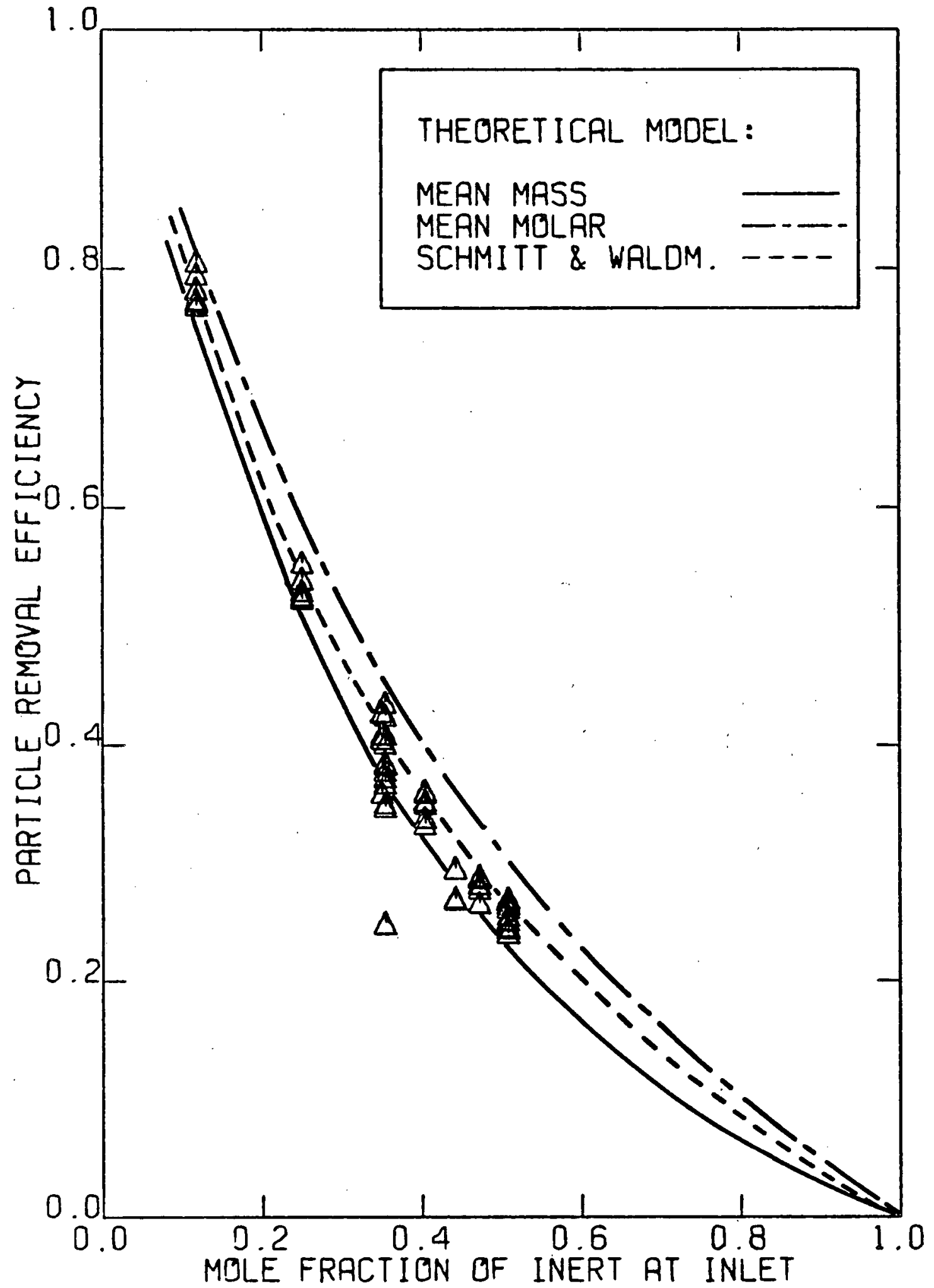

Figure 6.6 Results for nitrogen, ammonia, 0.79 micron diameter particles. Inlet flow rate of transferred gas held constant at $11.6 \times 10^{-4} \mathrm{~m} 3 / \mathrm{sec}$. 


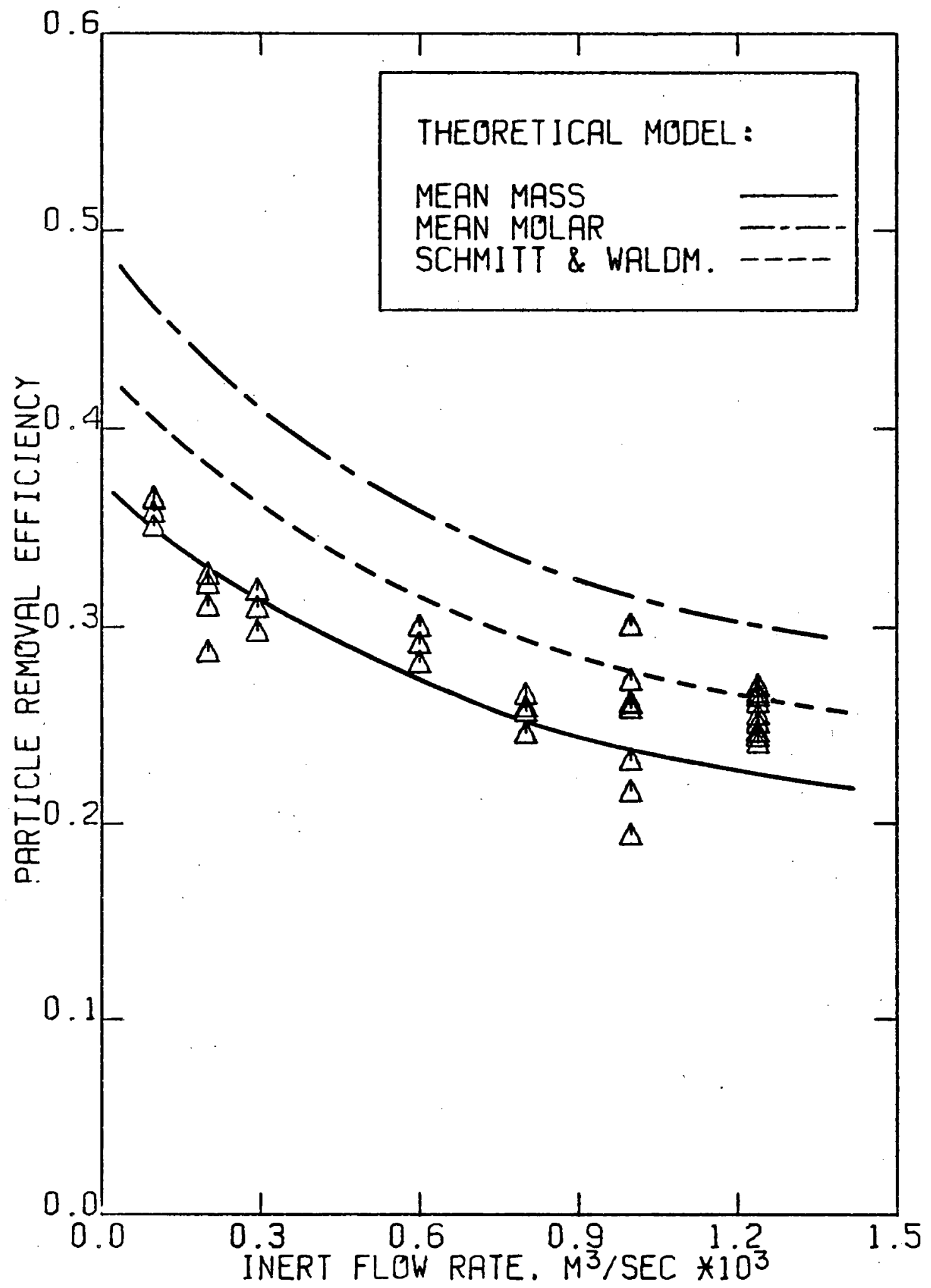

Figure 6.7 Results for nitrogen, ammonia, 0.79 micron diameter particles. Inlet gas composition held constant at 50 vo nitrogen. 


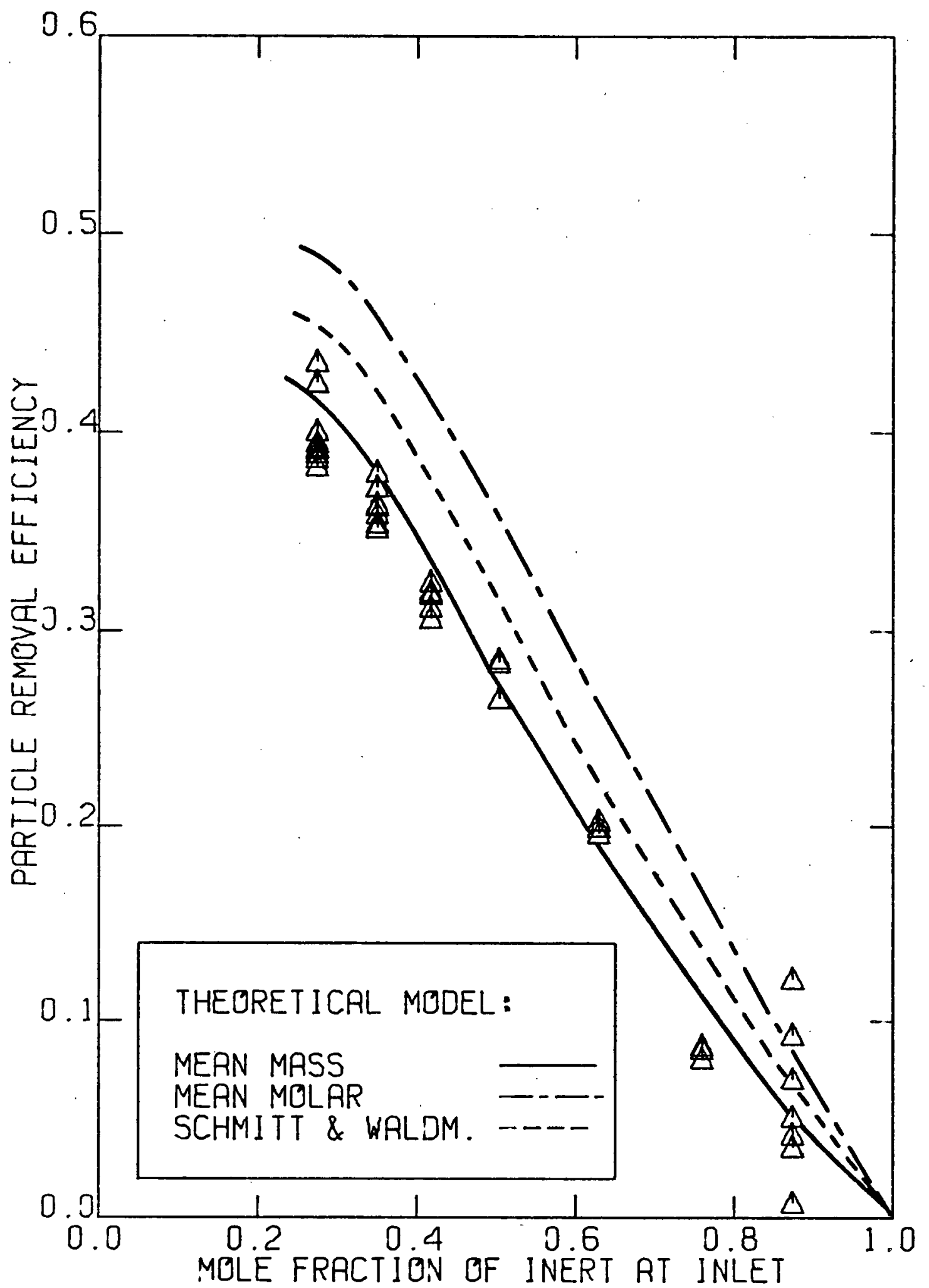

Figure 6.8 Results for nitrogen, ammonia, 1.011 micron diameter particles. Flow rate of inert gas held constant at $6.0 \times 10^{-4} \mathrm{~m}^{3} / \mathrm{sec}$. 


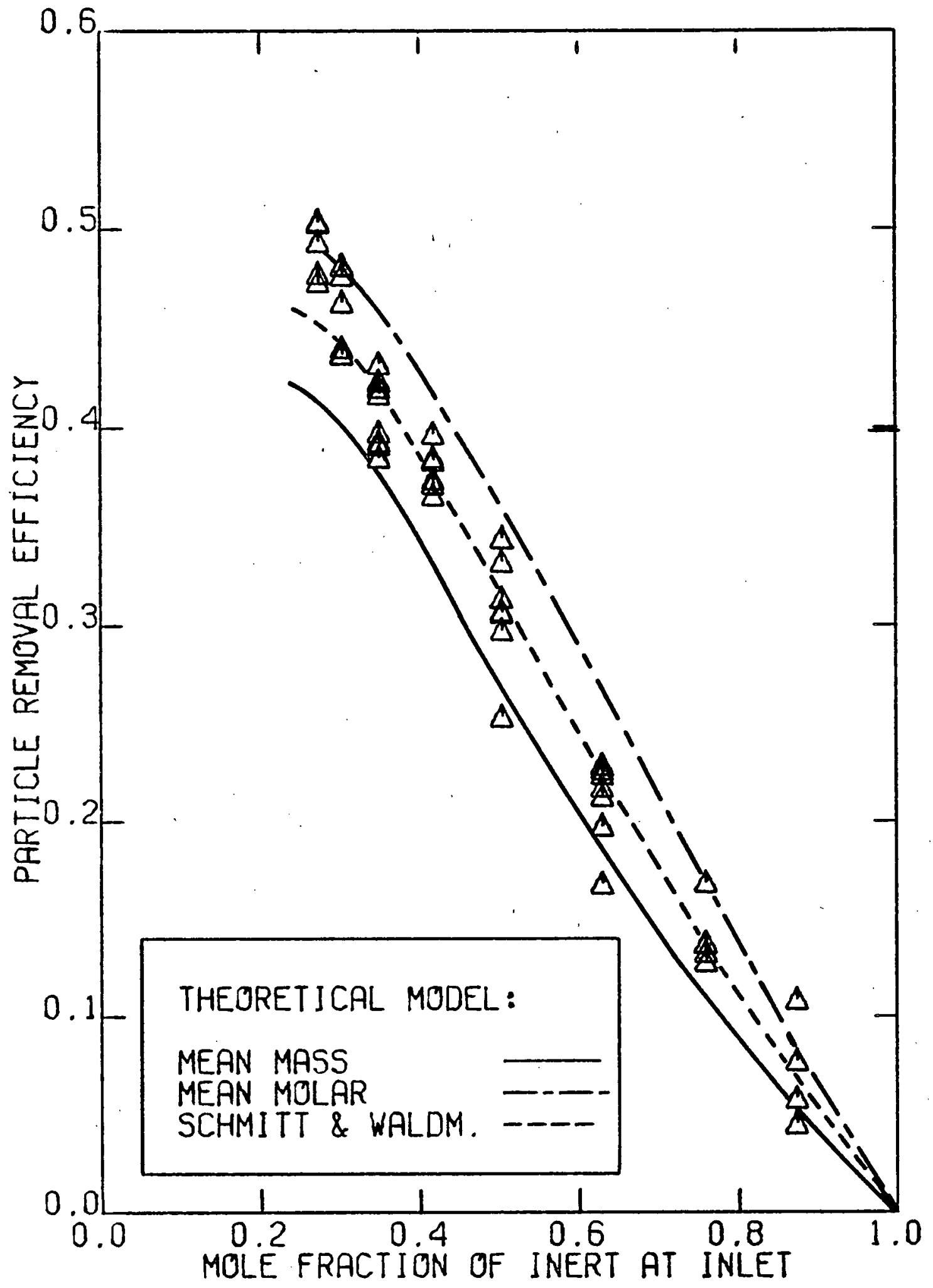

Figure 6.9 Results for nitrogen, ammonia, 2.02 micron diameter particles. Flow rate of inert gas held constant at $6.0 \times 10^{-4} \mathrm{~m}^{3} / \mathrm{sec}$. 


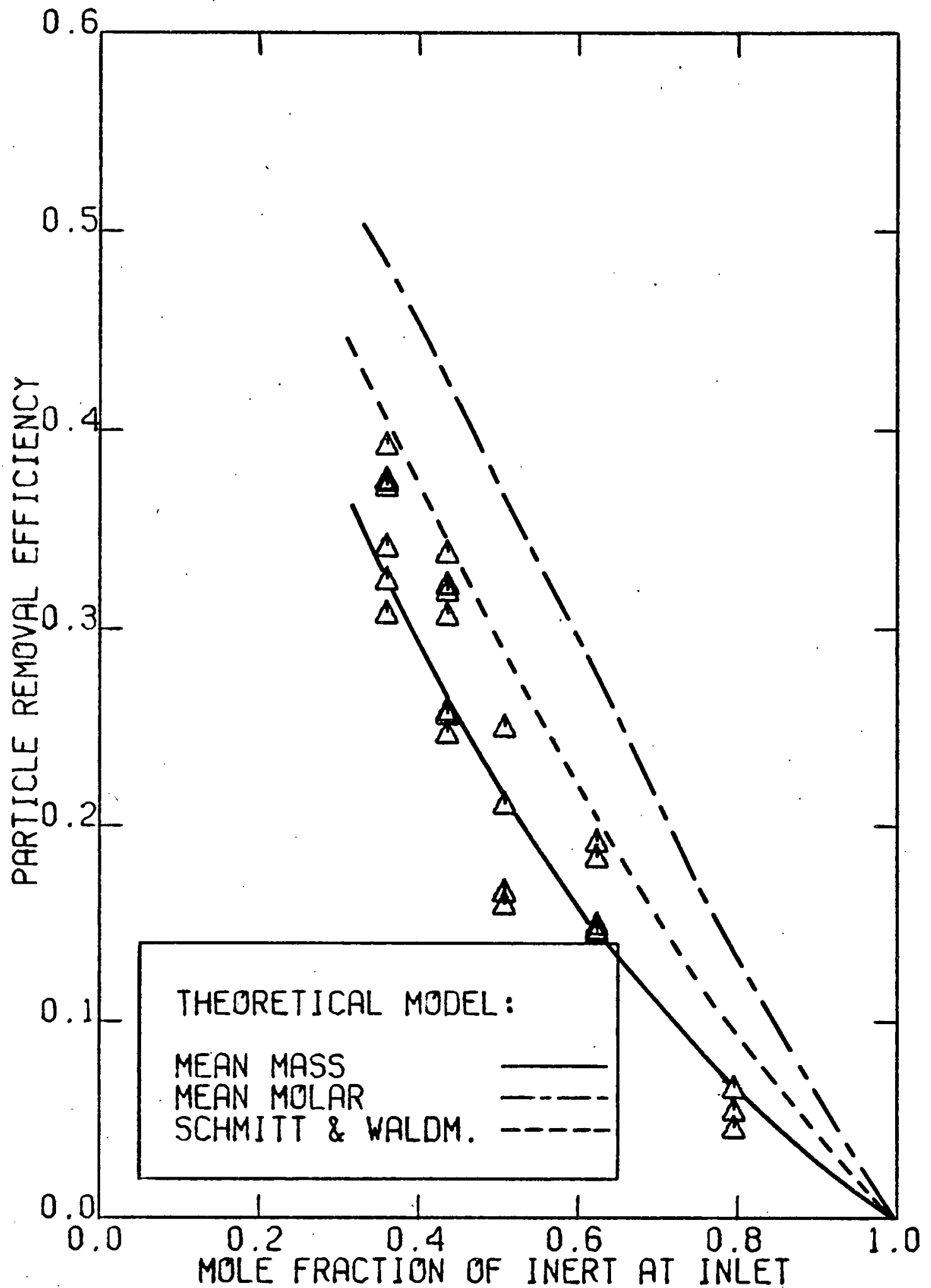

Figure 6.10 Results for argon, ammonia, 0.79 micron diameter particles. Flow rate of inert gas held constant at $5.6 \times 10^{-4} \mathrm{~m}^{3} / \mathrm{sec}$. 
141.

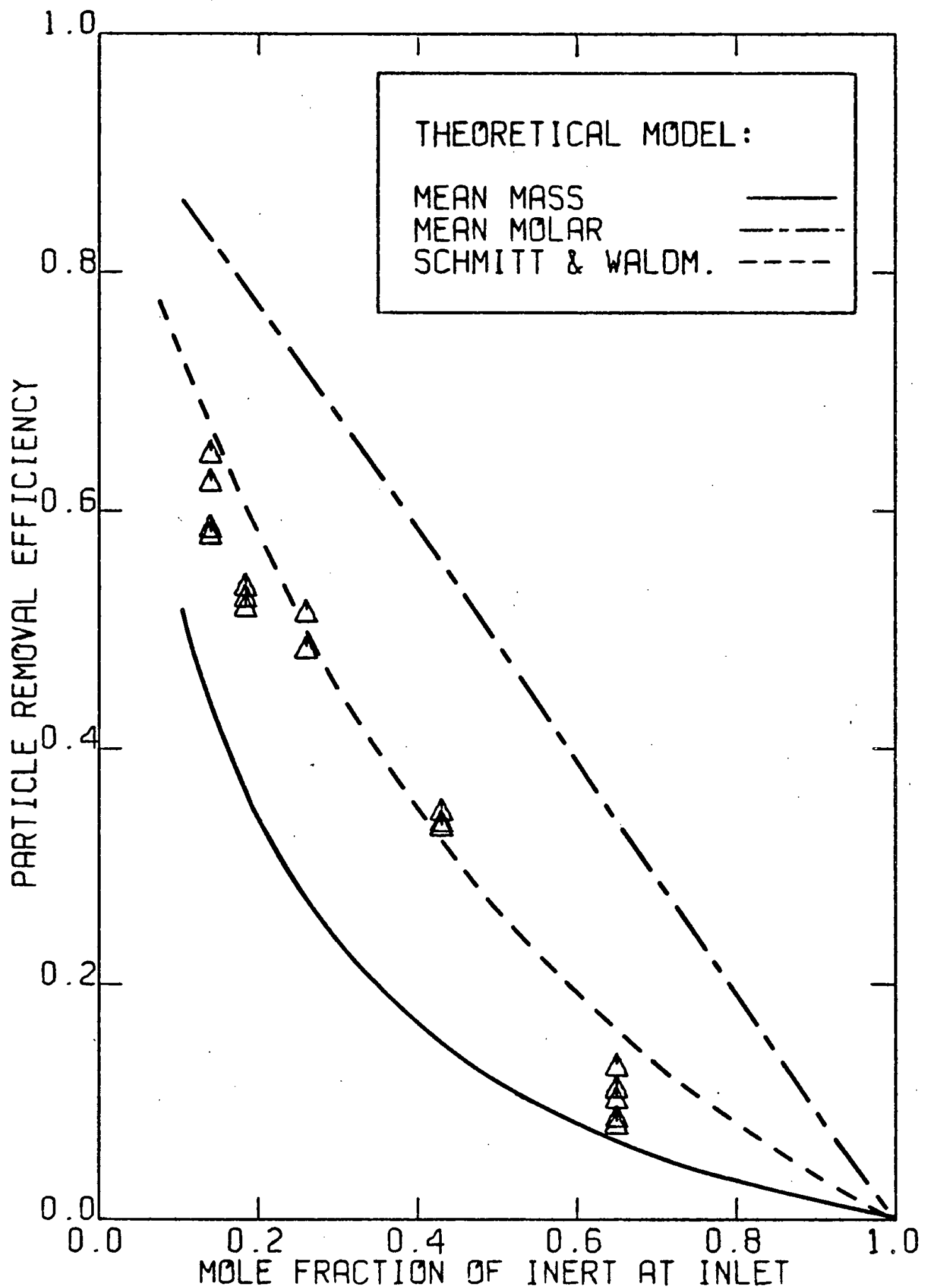

Figure 6.11 Results for freon $12\left(\mathrm{CF}_{2} \mathrm{Cl}_{2}\right)$, ammonia, 0.79 micron diameter particles. Flow rate of inert gas held constant at. $0.98 \times 10^{-4} \mathrm{~m}^{3} / \mathrm{sec}$. 


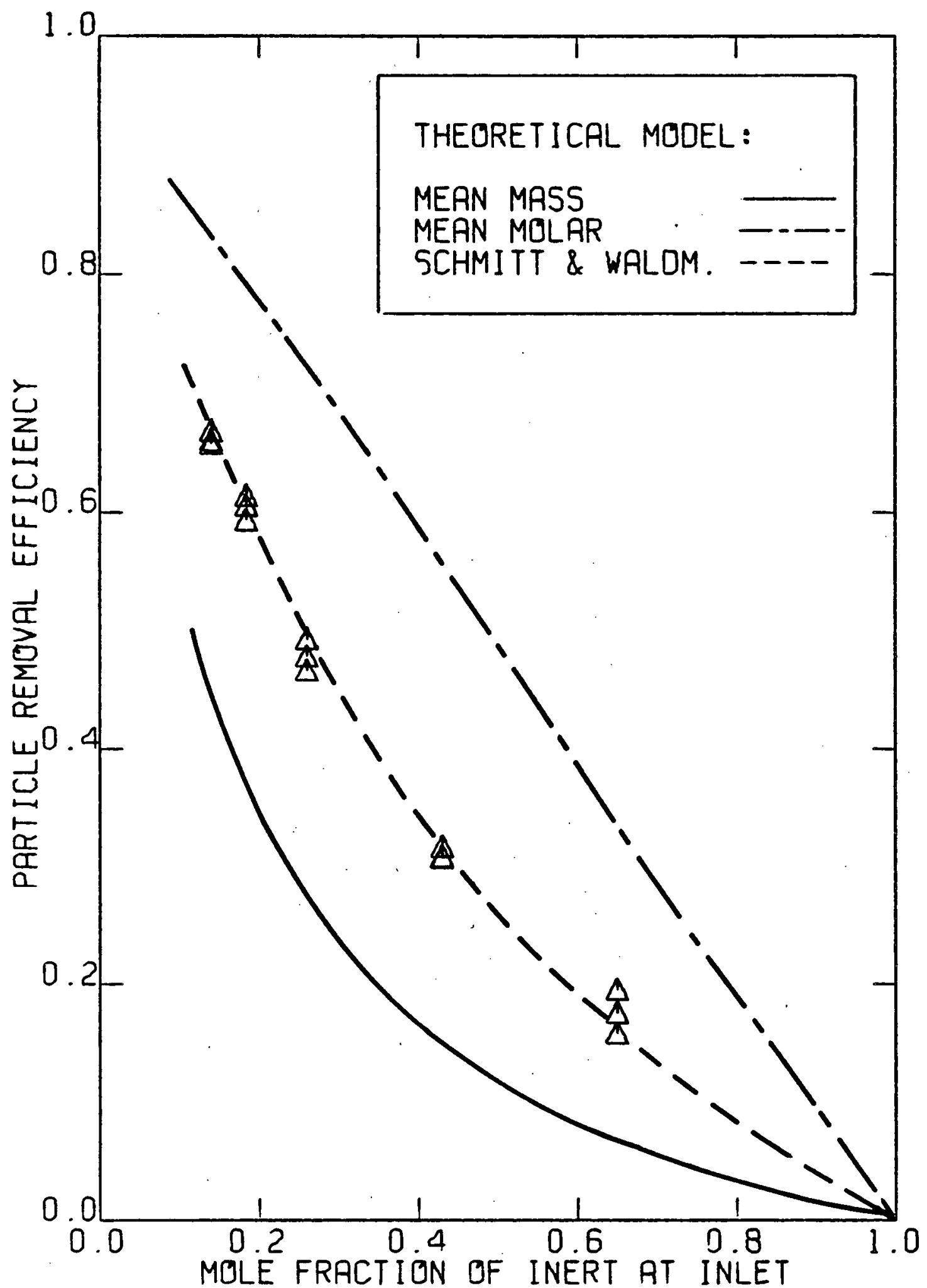

Figure 6.12 Results for freon $12\left(\mathrm{CF}_{2} \mathrm{Cl}_{2}\right)$, ammonia, 1.011 micron diameter particles. Flow rate of inert gas held constant at $0.98 \times 10^{-4} \mathrm{~m}^{3} / \mathrm{sec}$. 


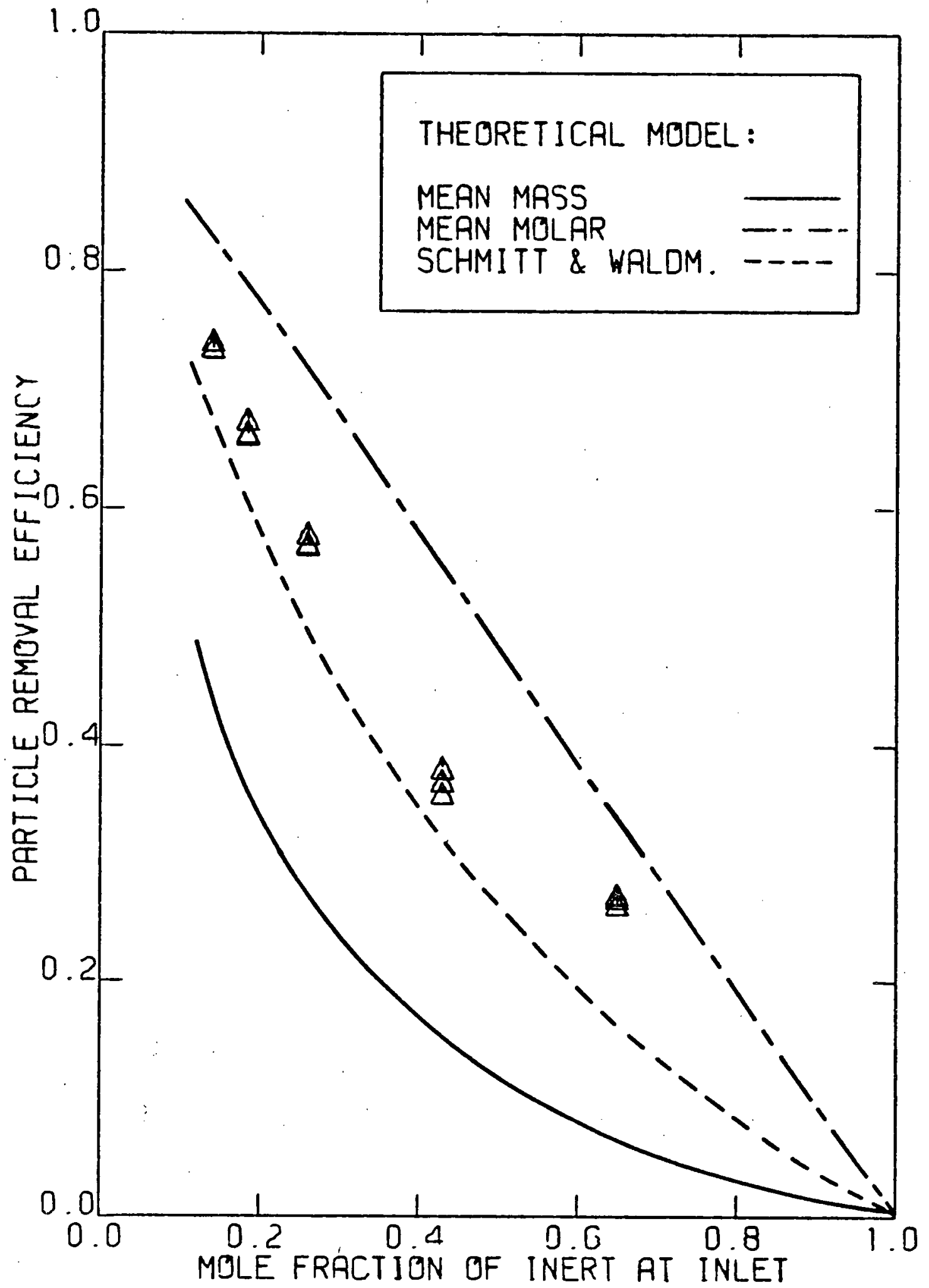

Figure 6.13 Results for freon $12\left(\mathrm{CF}_{2} \mathrm{Cl}_{2}\right)$, ammonia, 2.02 micron diameter particles. Flow rate of inert gas held constant at $0.98 \times 10^{-4} \mathrm{~m}^{3} / \mathrm{sec}$. 


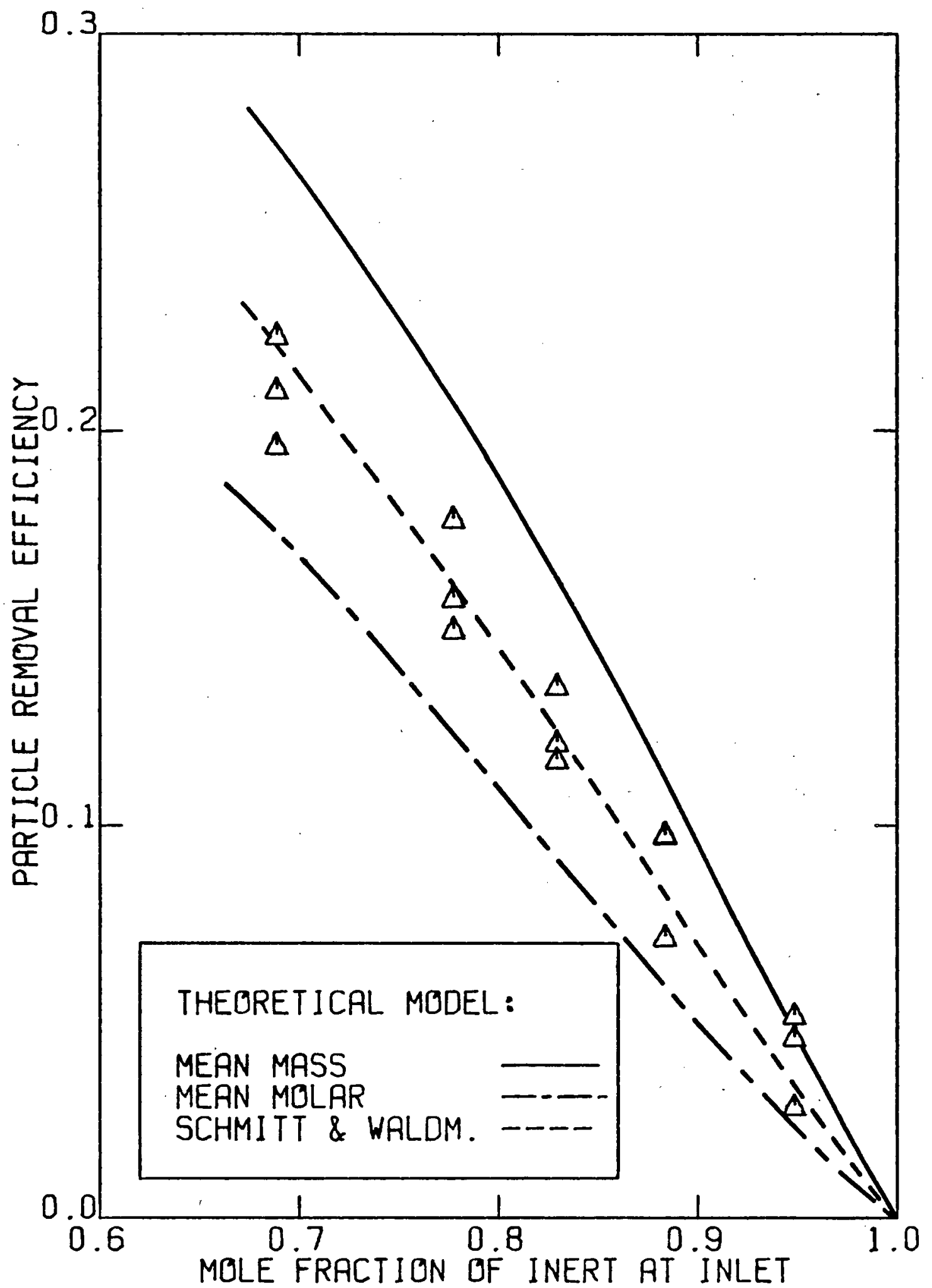

Figure 6.14 Results for nitrogen, trimethylamine $\left(\mathrm{N}\left(\mathrm{CH}_{3}\right)_{3}\right), 0.50$ micron diameter particles. Flow rate of inert gas held constant at $6.0 \times 10^{-4} \mathrm{~m}^{3} / \mathrm{sec}$. 
145 .

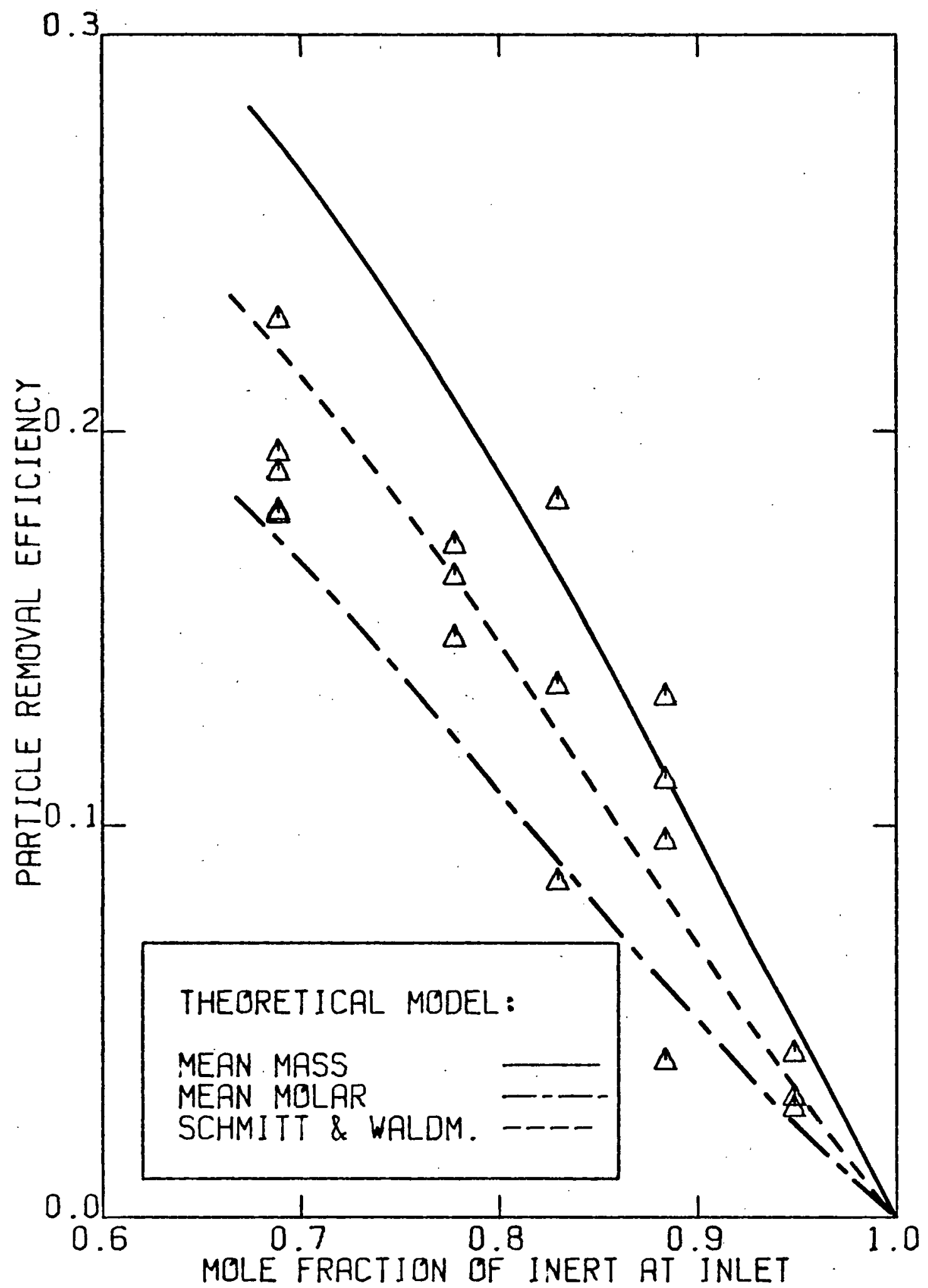

Figure 6.15 Results for nitrogen, trimethylamine $\left(\mathrm{N}\left(\mathrm{CH}_{3}\right)_{3}\right), 0.79$ micron diameter particles. Flow rate of inert gas held constant at $6.0 \times 10^{-4} \mathrm{~m}^{3} / \mathrm{sec}$. 


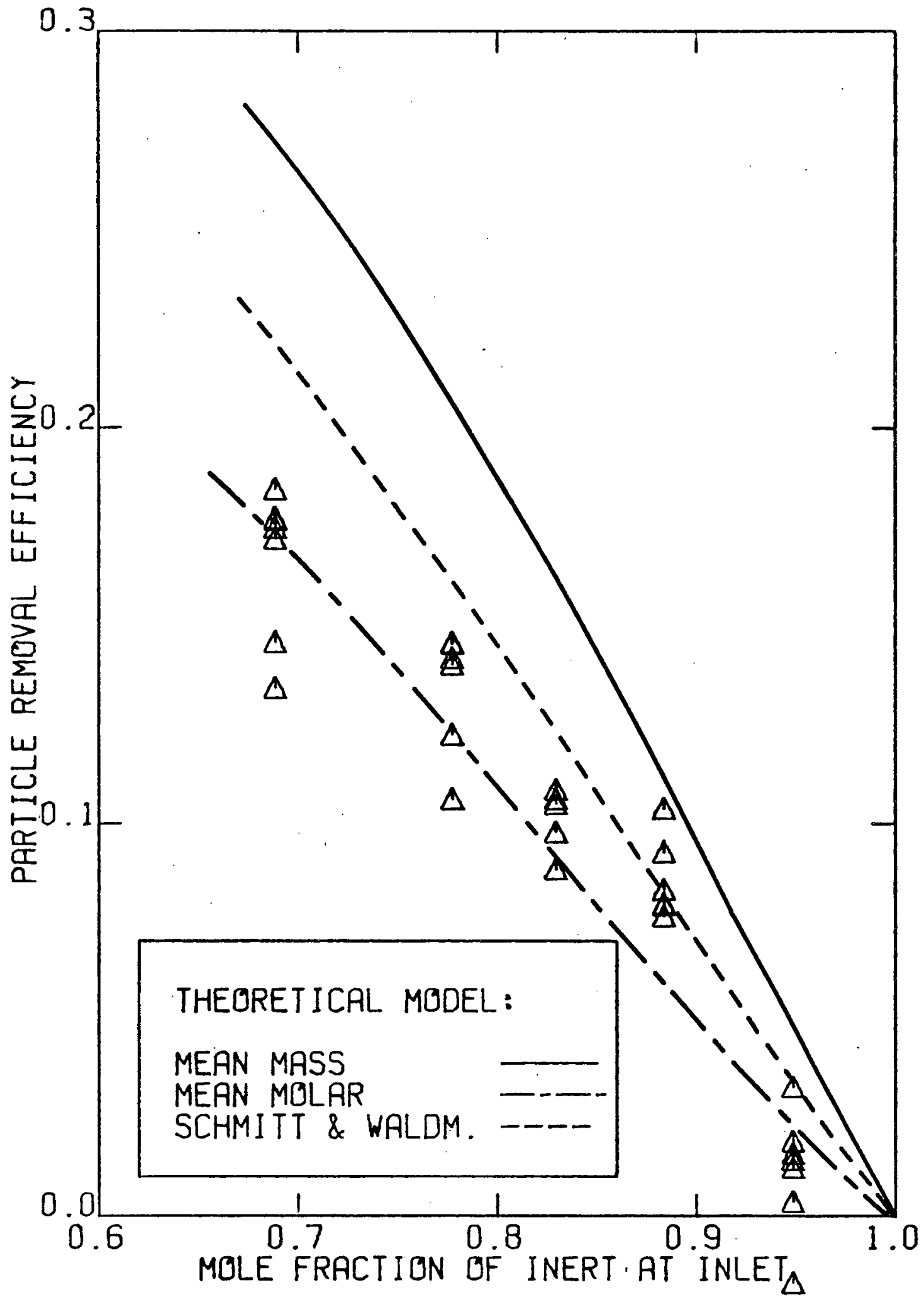

Figure 6.16 Results for nitrogen, trimethylamine $\left(\mathrm{N}\left(\mathrm{CH}_{3}\right)_{3}\right), 1.011$ micron diameter particles. Flow rate of inert gas held constant at $6.0 \times 10^{-4} \mathrm{~m}^{3} / \mathrm{sec}$. 
147.

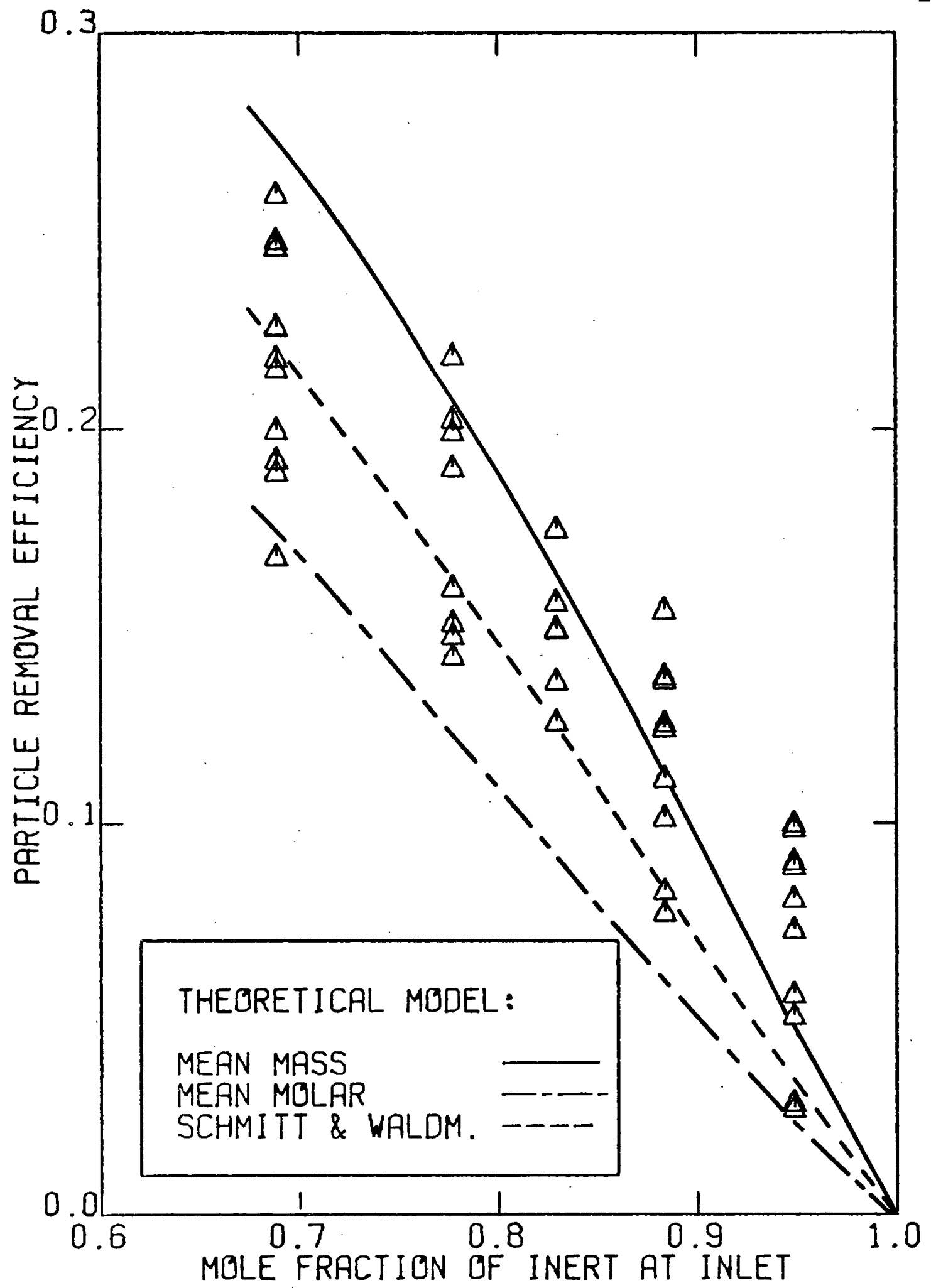

Figure 6.17 Results for nitrogen, trimethylamine $\left(\mathrm{N}\left(\mathrm{CH}_{3}\right)_{3}\right), 2.02$ micron diameter particles. Flow rate of inert gas held constant at $6.0 \times 10^{-4} \mathrm{~m}^{3} / \mathrm{sec}$. 


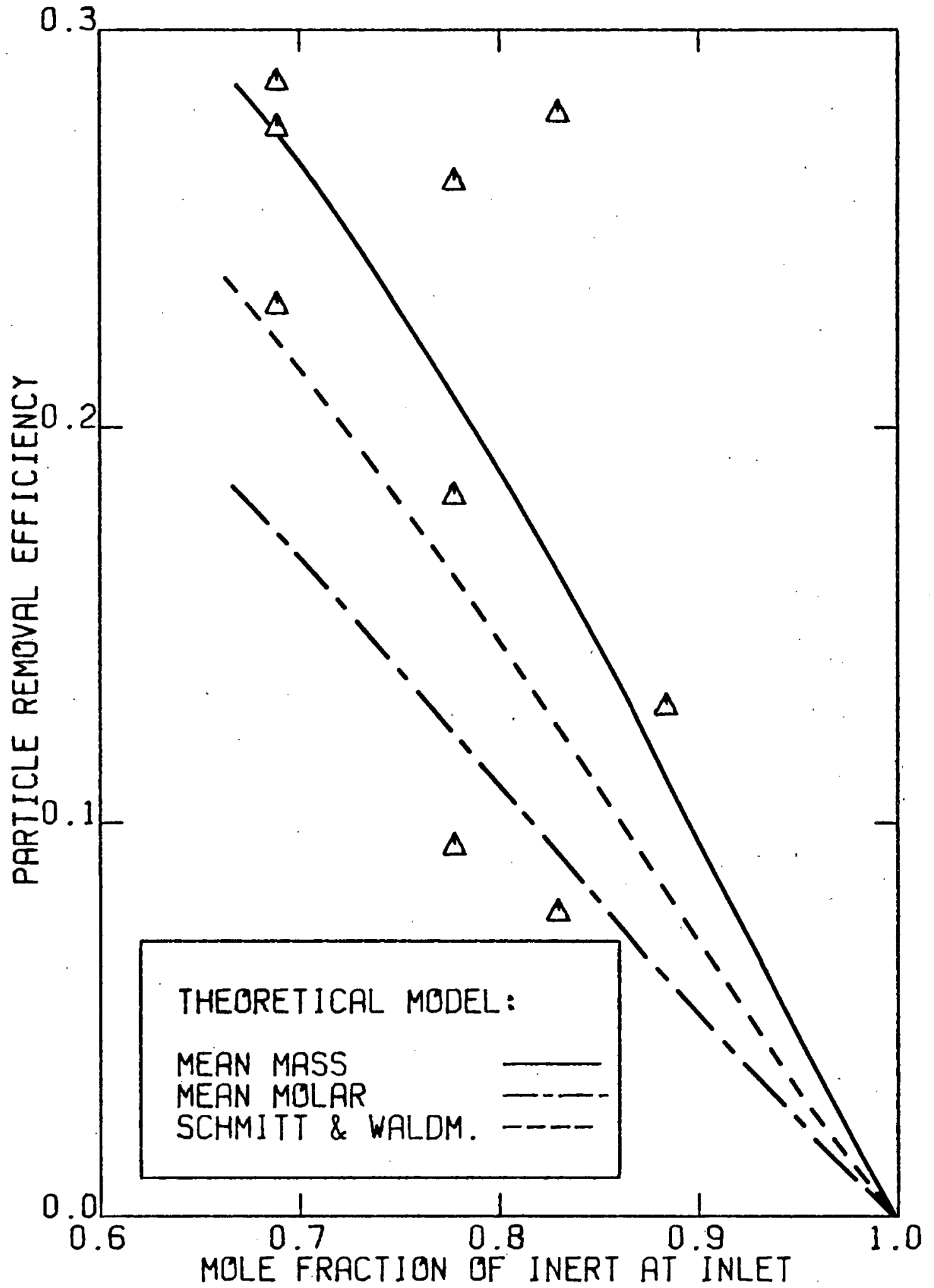

Figure 6.18 Results for nitrogen, trimethylamine $\left(\mathrm{N}\left(\mathrm{CH}_{3}\right)_{3}\right), 5.7$ micron diameter particles. Flow rate of inert gas held constant at $6.0 \times 10^{-4} \mathrm{~m}^{3} / \mathrm{sec}$. 


\section{Chapter 7}

\section{PRACTICAL APPLICATION}

\subsection{General}

It has been established in this study that diffusiophoresis can be an effective method for removing micron-sized particles from turbulent gas streams. However, its potential for practical application depends on its cost relative to other gas cleaning methods. Only the primary costs of heat for vapour generation and cooling water for vapour condensation are considered in the preliminary assessment which follows. These can be estimated from the latent heat of vaporization and rate of vapour usage.

The possibility of using diffusiophoresis to enhance and/or supplement particle removal by another mechanism is also discussed. However, no economic assessment is possible in this case without specific knowledge of the actual application, and a better understanding of the importance of interactive effects between mechanisms. 


\subsection{Investigations by Others}

Although there is no clear dividing line, some research has been oriented more towards developing practical methods for particle removal, than towards verifying fundamental theory or demonstrating the gas cleaning ability of diffusiophoresis under simple conditions. Often in these practical studies, diffusiophoresis is only one of a number of removal mechanisms operating. The brief summary of previous studies given in Table VI is not intended to be comprehensive, but rather to indicate the type of work that has been done by others.

It is apparent from these studies that the influence of diffusiophoresis was not well understood in many instances. Some workers never mentioned it, or gave it only cursory attention. Others reached conflicting conclusions about its importance, even when experimenting with similar equipment. This uncertainty is not surprising considering the variety of mechanisms by which vapour condensation from a dusty gas may influence particle removal.

\subsection{Optimal Vapour Use}

Particles will be removed from a mixture of a vapour and an inert gas, when the vapour is transferred out of the gas phase. The particle removal efficiency obtained can be increased by increasing the initial vapour concentration in the mixture. However, the improvement becomes less with each extra unit of 
TABLE VI

Enhancement of Particle Capture by Vapour Condensation

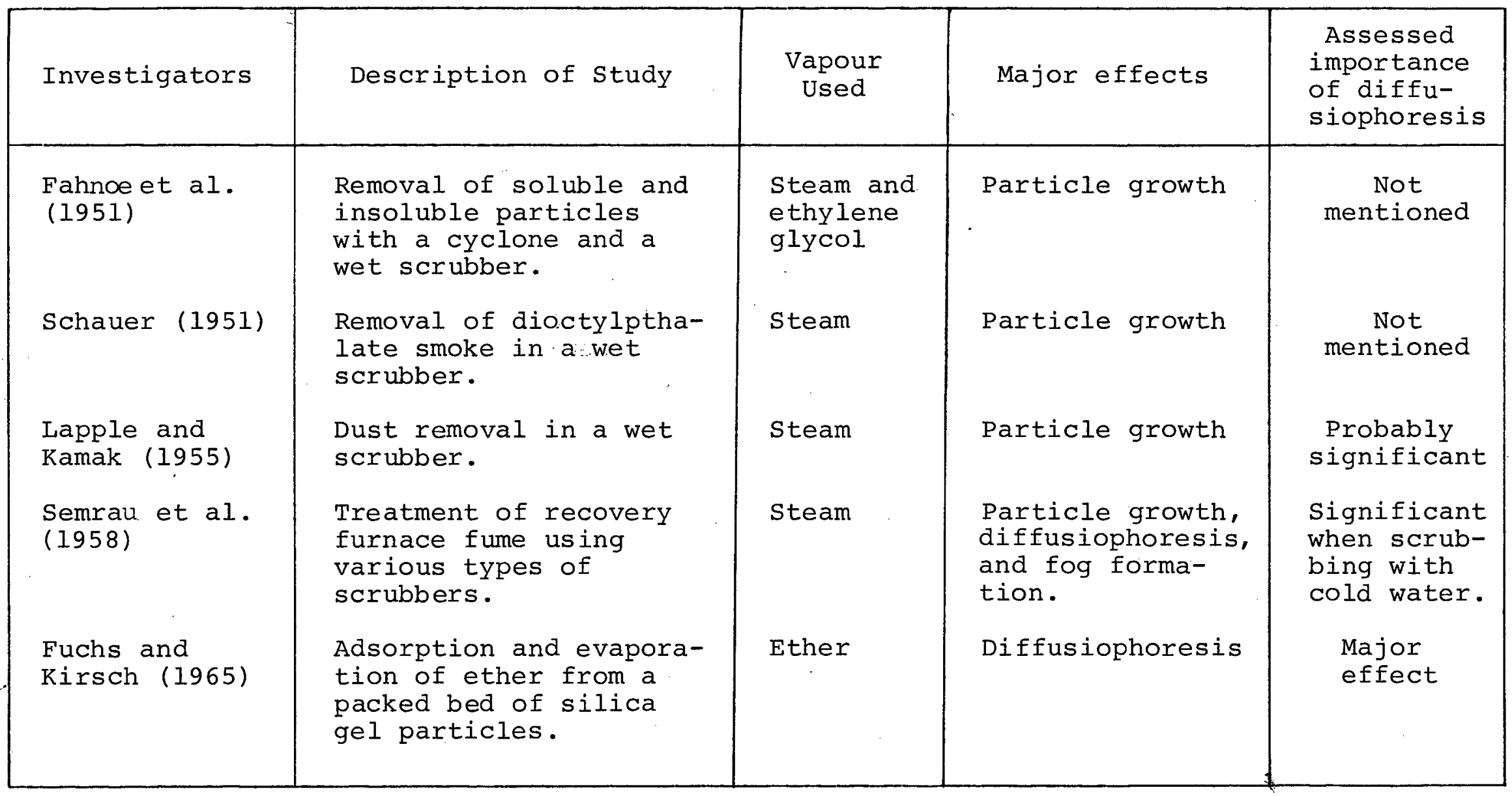


TABLE VI/continued ....

\begin{tabular}{|c|c|c|c|c|}
\hline Investigators & Description of Study & $\begin{array}{l}\text { Vapour } \\
\text { Used }\end{array}$ & Major effects & $\begin{array}{l}\text { Assessed } \\
\text { importance } \\
\text { of diffu- } \\
\text { siophoresis }\end{array}$ \\
\hline Litvinov (1967) & $\begin{array}{l}\text { Particle removal with a } \\
\text { venturi tube and sieve } \\
\text { tray scrubber. }\end{array}$ & Steam & Coagulation & $\begin{array}{l}\text { Only impor- } \\
\text { tant for } \\
\text { small par- } \\
\text { ticles } \\
(<0.1 \mu \mathrm{m})\end{array}$ \\
\hline $\begin{array}{l}\text { Rozen and } \\
\text { Kostin (1967) }\end{array}$ & $\begin{array}{l}\text { Particle removal in a } \\
\text { column with alternate } \\
\text { evaporation and con- } \\
\text { densation plates. }\end{array}$ & Steam & Particle growth & Negligible \\
\hline $\begin{array}{l}\text { Sparks and } \\
\text { Pilat }(1970)\end{array}$ & $\begin{array}{l}\text { Theoretical study of } \\
\text { the influence of diffu- } \\
\text { siophoresis on particle } \\
\text { collection in scrubbers. }\end{array}$ & Steam & - & $\begin{array}{l}\text { Has a sig- } \\
\text { nificant } \\
\text { influence } \\
\text { on particle } \\
\text { capture by } \\
\text { water drop- } \\
\text { lets. }\end{array}$ \\
\hline $\begin{array}{l}\text { Truitt and } \\
\text { Davis (1971) }\end{array}$ & $\begin{array}{l}\text { Particle removal in a } \\
\text { tubular condenser } \\
\text { scrubber. }\end{array}$ & Steam & Various effects & Significant \\
\hline
\end{tabular}

continued 
TABLE VI/continued ...

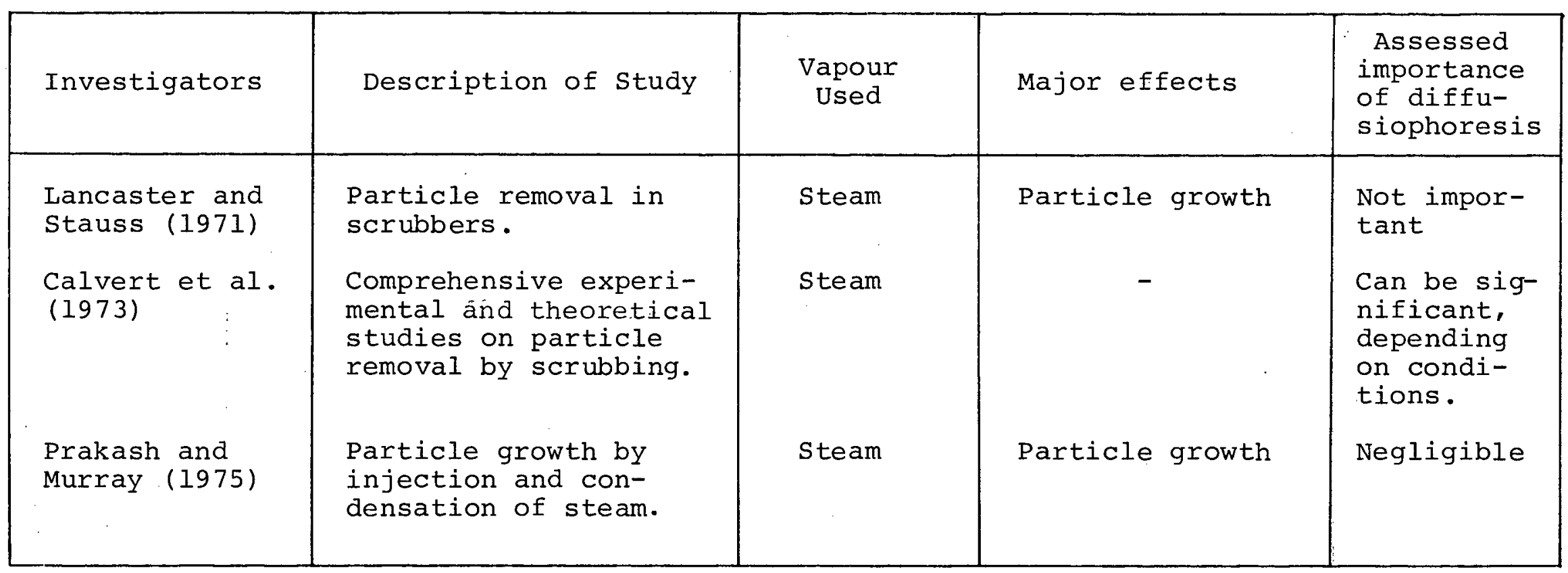


vapour added, because the inert gas is diluted, and the particle concentration reduced. It is therefore preferable to add and remove smaller quantities of vapour in several separate stages. The limiting case, which gives the optimal result, is to continuously add and remove vapour all along the inert gas flow path. This could be achieved, for example, when the inert gas flows between two parallel plates. Steam is diffused into the gas through one of these plates and condensed out at the other. The derivation of the theory for continuous vapour addition under turbulent conditions is similar to the case where all the vapour is added initially (see Chapter 4). Using the same notation, a particle balance over an elemental length of the collector yields

$$
n v_{p} a d z=-d\left(\frac{G n}{c}\right)
$$

where a refers only to the collector area per unit length. For developed conditions, when the cross flow of vapour between its source and sink is fully established, G is constant and equal to $\mathrm{G}_{1} / \gamma_{1} \cdot$ Hence

$$
\frac{d n}{n}=-\frac{\gamma_{1} v_{p} c}{G_{1}} a d z
$$

In the remainder of this section it is assumed that the particles move with the local mean mass velocity of the gas. Similar 
analyses can readily be made for other cases. Substituting for $v_{p}$ the expression for mean mass velocity (Equation 3.68) and integrating gives

$$
\frac{\mathrm{n}_{\text {out }}}{\mathrm{n}_{\text {in }}}=\exp \left\{-\frac{\mathrm{r}_{1} \mathrm{M}_{2} \mathrm{~N}_{2}}{\mathrm{MG}_{1}} \text { az }\right\} \text {, }
$$

where $\mathrm{Z}$ is the collector length. If $\mathrm{G}_{2}$ denotes the total molar cross flow of the diffusing component, then

$$
\varepsilon_{p}=\frac{n_{\text {in }}-n_{\text {out }}}{n_{\text {in }}}=1-\exp \left\{-\frac{\gamma_{1} M_{2} G_{2}}{M_{1}}\right\}
$$

When the vapour concentration is small, (i.e. $\gamma_{1}=1$ ), the exponent is equal to the mass of vapour used per mass of inert gas treated.

The importance of the method of vapour addition can be illustrated by calculating the vapour requirements to achieve 95\% particle removal. This can be done for continuous vapour addition using the theory just derived, and for a stagewise process using the theory developed in Chapter 4 . For simplicity the vapour concentration is assumed to be small in the case of continuous vapour addition. In the stagewise process the vapour is taken to be divided evenly between stages, and to be completely absorbed in each stage. The results are summarized in Table VII. In contrast to this, one can show that for laminar flow between parallel plates whose length is much greater than 
TABLE VII

Vapour Requirements to Attain 95\% Particle Removal

\begin{tabular}{|c|c|c|}
\hline $\begin{array}{c}\text { Total number } \\
\text { of stages }\end{array}$ & $\frac{\text { mass of vapour per stage }}{\text { mass of inert gas treated }}$ & $\frac{\text { total mass of vapour used }}{\text { mass of inert gas treated }}$ \\
\hline 1 & 19 & 19 \\
2 & 3.5 & 6.9 \\
3 & 1.7 & 5.1 \\
4 & 1.1 & 3.5 \\
infinity & - & 3.0 \\
\hline
\end{tabular}

* This is equivalent to continuous vapour addition with low vapour concentration. 
the inter-plate spacing, the particle removal efficiency becomes $100 \%$ when the cross flow of vapour between the plates is given by

$$
\mathrm{G}_{2}=\mathrm{MG}_{1} / \gamma_{1} \mathrm{M}_{2}
$$

Thus, when the vapour concentration is small (i.e. $\gamma_{1} \simeq 1$ ), all particles are removed using unit mass of vapour per unit mass of inert gas. Under turbulent conditions, the random nature of the fluid motion precludes the possibility of complete particle removal.

\subsection{Operating Cost Calculation}

For comparison with other particle removal methods, the operating costs are now calculated for treating particlebearing air with unit mass of steam per mass of air. With continuous vapour addition at low vapour concentrations this will remove $63 \%$ of the particles if they adopt the mean mass velocity of the gas. The costs. for steam and cooling water are taken as $\$ 2 / 1000 \mathrm{lb}(\$ 44 / \mathrm{MKg})$, and $2 \xi / 1000$ us gal $\left(0.53 \xi / \mathrm{m}^{3}\right)$, respectively. Twenty pounds of cooling water are assumed to be necessary for condensing 1 pound of steam. The cost of treating $1000 \mathrm{scf}$ of air using $1 \mathrm{lb}$ of steam per pound of air can then be calculated. 


$\begin{array}{llll}\text { steam, } 75.31 \mathrm{~b}: & 15 & \text { cents } \\ \text { cooling water, } 15061 \mathrm{~b}: & \frac{0.36 \text { cents }}{15.4 \text { cents }} \\ \text { total cost: } & & \frac{15}{}\end{array}$

(This is equivalent to $0.54 \mathrm{c} / \mathrm{m}^{3}$ air.) These costs are more than an order of magnitude higher than those for commercial particle collectors operating with similar efficiencies (Perry (1973)).

\subsection{Reduction of Heat Requirements}

The operating costs for particle removal by diffusiophoresis are primarily a function of the cost of vapour generation. This can be reduced by using a process that allows some heat recovery, or by using a vapour with a lower latent heat than water.

Heat recovery can be achieved in a multistage system, such as that shown in Figure 7.1 , where water vapour is used with lithium chloride solution as absorbent. Each stage consists of an evaporation and an absorption section. In the evaporative section of the first stage, vapour is produced by bringing the inlet gas into contact with a liquid which has been heated by an external source. The gas-vapour mixture then passes to the absorption section, where part of the vapour is removed by being brought into contact with the cooled liquid leaving the evaporator. The gas then flows into the second stage, which operates in a similar manner, but at a lower temperature. The heat extracted from the liquid leaving the first stage evaporator is used to heat the Iiquid fed to the second stage evaporator, thus removing 
water cycle

(stage 3)

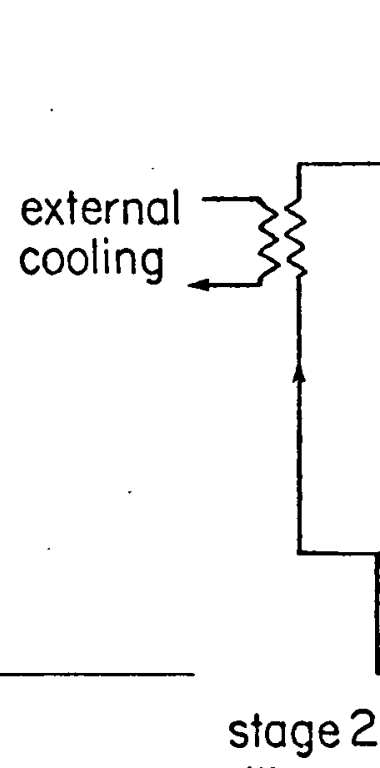

low concentration $\begin{gathered}\text { dilute } \\ \text { stream }\end{gathered}$

$\mathrm{LiCl}$ cycle

(stage 2)

interstage heat exchanger

high concentration

$\mathrm{LiCl}$ cycle

(stage 1)

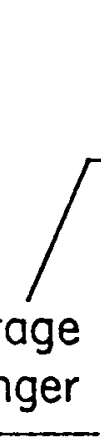

outlet

gas
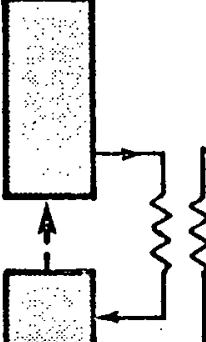

A

r 
the need for external heat in the second and subsequent stages. Finally, external cooling is used to remove heat from the liquid leaving the evaporator in the last stage.

The use of water is restricted not only by its high latent heat of vaporization, but also because most practical absorption systems cannot reduce the water vapour concentration in the gas to low levels at temperatures higher than about $100^{\circ} \mathrm{C}$. This fact reduces the number of stages that can be used with heat recovery :

Many organic liquids have much lower latent heats than water, some being less than $100 \mathrm{Btu} / \mathrm{lb}$ (233 Joule/g) compared to about $1000 \mathrm{Btu} / \mathrm{lb}$ (2330 Joule/g) for water. However, pure liquids are only useful over the temperature range at which they exert significant vapour pressures and yet can be easily absorbed and regenerated. This suggests the use of a mixture of liquids of different boiling points, both to provide the vapour and to act as the absorption liquid. The method of operation could be the same as that described earlier for a multistage system. The high boiling point components are concentrated in the first stage, and the boiling points successively decrease in later stages. In essence this process is similar to passing a dusty gas up through a column in which a multicomponent distillation takes place. However, the absorption of the less volatile components and the evaporation of the more volatile components must be carried out separately in each stage, otherwise the diffusiophoretic velocities will be small, and may even be directed so as to impede particle collection. 
7.6 Multiple Removal Mechanisms

Diffusiophoresis may form a useful adjunct to another particle removal mechanism during gas cleaning, and could be used to increase the efficiency of some types of existing equipment. For example, when a humid gas is treated in a wet scrubber the normal inertial mechanisms are accompanied by diffusiophoretic effects, and the particle removal efficiency increases. The magnitude of this increase in the case where there are no interactions between removal mechanisms (i.e., the mechanisms are locally additive) can now be determined.

To examine this case it is useful to introduce the particle penetration, which is defined as

$$
P=1-\varepsilon_{p}
$$

A differential particle balance over a length of the flow path $\mathrm{d} z$ for the gas flowing through the equipment gives

$$
d n=n v_{p, I} a_{I} d z+n v_{p, I I} a_{I I} d z+\ldots \ldots
$$

where each term on the right represents the particle removal by one mechanism, $v_{p}, I^{\prime} v_{p, I I} \cdots$ being the particle velocities and $a_{I}, a_{I I}, \ldots$ being the effective transfer areas for each mechanism.

Integration of this expression yields 


$$
\frac{\mathrm{n}_{\text {out }}}{\mathrm{n}_{\text {in }}}=\mathrm{P}=\mathrm{P}_{\text {I }} \mathrm{P}_{\text {II }} \mathrm{P}_{\text {III }} \ldots \ldots \ldots
$$

where each penetration must be expressed on the same basis, e.g., particles per mole of inert fluid. Thus the total penetration equals the penetration obtained if the fluid passes through a series of stages, in each of which only one removal mechanism, operates. The predicted total penetration is generally valid for one particle size and density, except in the case of thermoand diffusiophoretic removal, where these factors have no influence outside the transition regime. Thus, when the conditions required for the particle removal theory hold (see Chapter 4), it is straightforward to determine the amount of removal due to diffusiophoresis in the presence of other mechanisms.

\subsection{Interactions between Mechanisms}

The theory in the previous section is contingent on the condition that interactions between removal mechanisms do not occur. This is not always ; the case. For example, the work of Annis and Mason (1975) indicates that, when a small particle simultaneously undergoes thermo- and diffusiophoresis, the particle velocity is not simply the sum of the velocities caused by the individual mechanisms, but involves also a small interactive term. Second-order interactive effects such as this have little influence on particle collection efficiency, 
and are therefore mainly of academic interest.

It is suggested in this study, however, that under certain conditions a significant interaction may exist between inertial deposition and other removal mechanisms, such as diffusiophoresis. This situation can arise when the major resistance to inertial removal is in particle transport across the momentum transfer boundary layer, rather than in transport from the turbulent core into the boundary layer. In these circumstances, any mechanism such as diffusiophoresis, which can assist in carrying particles across the boundary layer, should substantially enhance the rate of inertial deposition and extend the influence of the inertial mechanism to smaller particle sizes. The possibility of such an interaction appears not to have been recognized by others.

Early deposition models such as those of Friedlander and Johnstone (1957), Davies (1966), and Beal (1970), were too crude for the possibility of interaction to be detected. They would in fact predict reduced removal, as both mechanisms compete for particles from the turbulent core, thereby lowering the particle concentration at the edge of the boundary layer. The paper of Hutchinson et al. (1971) provides a more detailed model. It predicts that under certain conditions the concentration of particles can reach a peak near the transfer surface. This occurs when the fraction of particles which approach the surface is large, but the fraction able to penetrate the boundary layer and reach the surface is small. This behaviour has been observed experimentally. Under these conditions even a small 
diffusiophoretic effect could provide a means of conveying particles across the boundary layer and thus completing the removal process.

The experimental conditions in this study were chosen so that inertial deposition would be negligible, and consequently no interactive effects were observed. By contrast, in the work of Azarniouch et al. (1975) both inertial and diffusiophoretic removal were significant. They used the method of Hutchinson et al., to predict that the resistance to inertial deposition lay virtually entirely in particle transport across the boundary layer. Thus, strong interaction should be expected, since even a small diffusiophoretic velocity will move a particle across the boundary layer, once it is deposited there by inertial mechanisms. Azarniouch et al. did not reach these conclusions, and make no mention of possible interactions.

Furthermore, their experimental results do not confirm this prediction since all efficiencies were considerably less than unity, and some were as low as 0.4. In view of the criticisms of their experimental work made earlier, it is not possible to draw any definite conclusions regarding interactions from these results. However, it appears as if the interactive effects are smaller than the Hutchinson model indicates. This probably reflects the fact that the model, although more refined than previous ones, still does not fully describe the behaviour in the boundary layer. The possibility allowed by the model, that virtually all particles can be removed from the core and concentrated in the boundary layer, is not realistic. Even in the boundary layer eddies must 
still play some role, and in particular must provide a mechanism for returning particles to the gas core. It is expected that a more accurate model would predict a lower enhancement of removal by interactive effects.

If indeed there are strong interactive effects between inertial and diffusiophoretic removal mechanisms, this could have important implications in the design of certain particle removal equipment. However, because of lack of adequate theory and experimental data, no definite conclusions can be reached at present about the existence of such effects. 
Chapter 8

\section{SUMMARY AND CONCLUSIONS}

\subsection{Fundamental Theory}

Expressions for the velocity of a small particle in an undisturbed diffusing gas mixture have been derived by several workers. They are all substantially the same, and give satisfactory agreement with experimental measurements. However, for large particles, two different expressions have previously been proposed (ignoring second-order terms). These are derived from theories which invoke or imply the existence of diffusion slip at the particle surface.

In the current study the continuum mechanics equations were solved in a more rigorous manner than was hitherto available. The gas was taken to be at rest at the surface of the particle, since significant slip was shown to violate the condition of energy conservation. Hence, the particle was shown to adopt the mean mass velocity of the gas. Inadequate experimental Wrk existed to substantiate this result. 


\subsection{Derived Theory}

A theory was developed to predict the diffusiophoretic particle removal caused by diffusional mass transfer from a multicomponent gas stream. Two separate derivations were made. The first assumes that mass transfer takes place from a turbulent and well-mixed gas core across a laminar film adjacent to the transfer surface. The second is more rigorous, since it is based solely on the non-steady-state forms of the continuity equations for the particle and for the gas mixture. It is therefore independent of the patterns of mass transfer or gas flow. The two derivations gave identical results.

The theoretical predictions for particle removal depend on the value of the local particle velocity. It was found that if the particles adopt the local mean mass velocity of the fluid (as predicted for large particles in the present study), then the fractional removal of particles is identical to the mass fraction of the gas transferred. Similarly, if they adopt the local mean molar velocity of the fluid, then the removal efficiency equals the molar fraction of gas transferred. A simple result was also obtained when the particles were assumed to travel at the velocity predicted by Schmitt and Waldmann (1960) .

Azarniouch et al. (1975) derived a similar theory for particle removal by diffusiophoresis, and analogous theories have been presented for thermophoresis by several authors. Both theories presented in the current study are more rigorous than 
any of these, and yield simpler results. However, no suitable experimental work was previously available against which to test them.

\subsection{Experimental Work}

Experimental studies were made of particle removal by diffusiophoresis from turbulent gas streams. A binary gas mixture containing aerosol particles was passed up through a wetted wall column (0.0254 m I.D. and $0.77 \mathrm{~m}$ in length), countercurrent to a flow of water. One component was insoluble, while the other was partially absorbed. The resulting particle removal was determined by measuring the inlet and outlet aerosol number concentrations in the gas. The soluble gases tested were ammonia and trimethylamine, while the insoluble gases were helium, methane, nitrogen, argon, and freon 12 (dichlorodifluoromethane). Uniform latex particles of $0.50,0.79,1.011,2.02$, and 5.7 microns in diameter were used.

The measured particle removal efficiencies were compared with three theoretical predictions based on the three velocity expressions mentioned previously. It was found that they always tended to lie between the predictions of the mean mass and mean molar velocity models. Also, the data for each experiment showed a similar trend to the three predictions, while generally not agreeing with any of them. It was therefore concluded that the theory of particle removal was substantially correct, but that the particles probably fell into the transition regime, 
in which none of the velocity expressions applied. Consequently, the fundamental theory for the velocity of large particles could not be definitively tested. It was, however, concluded that the mean molar velocity was not appropriate, since almost none of the data fell close to the corresponding theoretical predictions.

Attempts to confirm the existence of transition regime behaviour by statistically comparing the data with a transition model were unsuccessful. Previous studies which purport to show the extent of the transition regime indicate that large particle behaviour should have been expected in most of the current experiments. Those studies are, however, open to criticism. The extent of the transition regime, and the manner in which particle velocity varies with Knudsen number within this region, are difficult to predict because of the complex interactions of molecular species with the particle surface and with each other.

\subsection{Practical Application}

It has been established in this study that diffusiophoresis can be an effective method of removing micron-sized particles from turbulent gas streams. However, its potential for practical application depends on its cost relative to other gas cleaning methods. The transferred gas can conveniently be produced either by evaporation of a liquid or by desorption of the gas from solution. The primary costs are for heat required to carry out this operation, and for cooling to recondense the gas or remove the heat evolved during re-absorption. The way in 
which the transferred gas is used was found to have a large effect on the particle removal efficiency obtained, and hence on the cost.

The use of steam as the transferred gas was shown to be uneconomic except possibly in special circumstances, since the cost was an order of magnitude higher than for other removal methods. Reductions in cost may be possible through the use of heat recovery, or through the employment of gases which require less heat in their generation.

Diffusiophoresis could in some instances present a useful supplement to particle removal by other mechanisms. There is also some evidence that it may interact with the inertial deposition mechanism under certain conditions to give substantially enhanced particle removal. However, no satisfactory experimental work has been published against which to test this conjecture. 


\section{NOMENCLATURE}

Symbol

a

$a_{i}, a_{i}^{*}$

$\mathrm{a}_{\mathrm{p}}$

A

$A^{\prime}$

$\mathrm{A}_{r}$

$\mathrm{b}_{i}^{*}$

$\mathrm{B}^{\prime}$

${ }^{B} r$

C

$c_{i}$

$C^{\prime}$

$\mathrm{c}^{\prime}$

$c^{r}$

$\mathrm{c}_{i}^{\mathrm{r}}$
Explanation and typical units

interfacial area, $\mathrm{m}$.

coefficients dependent on interaction of gas molecules with surface.

particle acceleration, $\mathrm{m} / \mathrm{sec}$.

cross section area, $\mathrm{m}^{2}$.

constant in solution for stream function.

constant in expression for particle removal efficiency.

coefficient dependent on interaction of gas molecules with surface.

constant in solution for stream function.

constant in expression for particle removal efficiency.

molar concentration, $\mathrm{Kg} \operatorname{mole} / \mathrm{m}^{3}$.

partial molar concentration for species $i$, $\mathrm{Kg}$ mole $/ \mathrm{m}^{3}$.

general concentration.

partial general concentration for species $i$. root mass concentration, $(\mathrm{Kg})^{\frac{1}{2}}(\mathrm{Kg} \text { mole })^{\frac{1}{2}} / \mathrm{m}^{3}$. partial root mass concentration for species $i$, $(\mathrm{Kg})^{\frac{1}{2}}(\mathrm{Kg} \text { mole })^{\frac{1}{2}} / \mathrm{m}^{3}$. 
Symbol

$c^{\prime}$

$\mathrm{C}_{r}$

$a_{0}, d_{1}, d_{-1}$

D

$\mathrm{D}_{i}$

D'

$\mathrm{E}^{2}$

E

f

F

g

g

G

$\mathrm{G}_{1}$

$\mathrm{G}_{2}$

G'

i

k

$\mathrm{Kn}$

$\mathrm{m}_{i}$

M

$\mathrm{M}_{i}$

$\mathrm{M}_{\mathrm{p}}$

n

${ }^{n} 1$
Explanation and typical units

constant in solution for stream function.

constant in expression for particle removal efficiency.

parameters dependent on gas composition.

diffusivity of binary mixture, $\mathrm{m}^{2} / \mathrm{sec}$.

diffusivity of component $i$ in a multicomponent mixture, $\mathrm{m}^{2} / \mathrm{sec}$.

constant in solution for stream function.

vector operator, $/ \mathrm{m}^{2}$

energy dissipation rate, Joule/sec.

constant.

force on particle, $\mathrm{Kg} / \mathrm{m} / \mathrm{sec}^{2}$.

magnitude of gravitational acceleration, $\mathrm{m} / \mathrm{sec}^{2}$.

gravitational acceleration vector, $\mathrm{m} / \mathrm{sec}^{2}$.

molar flow rate of gas, $\mathrm{Kg}$ mole/sec.

molar flow rate of component $1, \mathrm{Kg}$ mole/sec.

molar cross flow rate of component 2 , $\mathrm{Kg}$ mole/sec.

constant in solution for $\mathrm{v}_{r}$.

gas component.

number of gas components.

Knudsen number.

molecule weight for species $i, \mathrm{Kg}$.

mean molecular weight, $\mathrm{g} / \mathrm{g}$ mole.

molecular weight for species $i, g / g$ mole.

particle mass, $\mathrm{Kg}$.

particle number concentration, \#/m 3 .

particle number concentration based on species 1 , $\# / \mathrm{m}^{3}$. 
Symbol

$\mathrm{N}_{\mathrm{i}}$

$\mathrm{N}_{\mathrm{i}}^{\mathrm{r}}$

$\mathrm{p}$

P

$r$

$r_{i}$

$r_{12}$

$\mathrm{R}$

$\mathrm{Re}$

S

Sc

$t$

$u_{w}$

V

$\underline{v}^{*}$

$\mathrm{v}^{r}$

$\underline{v}^{\prime}$

$v_{p}$

$\underline{\mathrm{v}}_{\mathrm{p}}$

$v_{r}, v_{\theta}$

$V_{\infty}$

$\mathrm{v}_{\text {slip }}$

W
Explanation and typical units

molar flux of species $i, \mathrm{Kg}$ mole $/ \mathrm{m}^{2} \mathrm{sec}$.

root mass flux of species $i,(\mathrm{Kg})^{\frac{1}{2}}(\mathrm{Kg} \text { mole })^{\frac{1}{2}} / \mathrm{m}^{2} \mathrm{sec}$.

pressure, $\mathrm{Kg} / \mathrm{m} \mathrm{sec}{ }^{2}$.

penetration.

radial coordinate, $\mathrm{m}$.

molecular radius for species $i, m$.

$\left(r_{1}+r_{2}\right) / 2, m$.

particle radius, $\mathrm{m}$.

Reynolds number.

stopping distance parameter, $\mathrm{m}^{2}$.

Schmidt number.

time, sec.

velocity of free flat, surface, $\mathrm{m} / \mathrm{sec}$.

mean mass velocity of fluid, $\mathrm{m} / \mathrm{sec}$.

mean molar velocity of fluid, $\mathrm{m} / \mathrm{sec}$.

mean root mass velocity of fluid, $\mathrm{m} / \mathrm{sec}$.

general velocity, $\mathrm{m} / \mathrm{sec}$.

particle velocity in $\mathrm{x}$ direction, $\mathrm{m} / \mathrm{sec}$.

particle velocity vector, $\mathrm{m} / \mathrm{sec}$.

fluid velocity components in body-centred coordinates, $\mathrm{m} / \mathrm{sec}$.

fluid velocity with respect to particle for large $r, \mathrm{~m} / \mathrm{sec}$.

slip velocity, $\mathrm{m} / \mathrm{sec}$.

mean mass velocity of gas with respect to number-centred coordinates, $\mathrm{m} / \mathrm{sec}$. 
$\underline{\text { Symbol }}$

$w_{0}$

X

Z

Z

$\alpha$

$\alpha^{\prime}$

$\alpha_{\mathrm{T}}$

$\beta_{i}$

$\gamma_{i}$

$\delta$

${ }^{\varepsilon} \mathrm{g}$

$\varepsilon_{\mathrm{p}}$

$\varepsilon_{\mathrm{MA}}$

${ }^{\varepsilon} \mathrm{SW}$

$\varepsilon_{\text {TR }}$

n

$\theta$

$\kappa$

$\mu$

$\rho$

$\rho_{0}$

$\rho_{i}$
Explanation and typical units

value of $\mathrm{w}$ for large $\mathrm{r}$ and $\theta=\pi / 2, \mathrm{~m} / \mathrm{sec}$. coordinate in direction of diffusion of undisturbed gas, $\mathrm{m}$.

coordinate in direction of fluid flow, $\mathrm{m}$. collector length, m.

slip coefficient.

slip coefficient redefined.

thermal diffusion factor.

accommodation coefficient for species $i$.

mole fraction of species $i$.

function of collision integrals

fraction of gas removed.

particle removal efficiency.

prediction of mean mass velocity model.

prediction of Schmitt and Waldmann model.

prediction of transition regime model.

dimensionless density gradient for large $r$.

polar coordinate.

bulk viscosity, $\mathrm{Kg} / \mathrm{m}$ sec.

shear viscosity, $\mathrm{Kg} / \mathrm{m} \mathrm{sec}$.

density of fluid, $\mathrm{Kg} / \mathrm{m}^{3}$.

density of fluid for large $r$ and $\theta=\pi / 2$, $\mathrm{Kg} / \mathrm{m}^{3}$.

partial density of component $i, \mathrm{~kg} / \mathrm{m}^{3}$. 
Symbol

$\underline{I}$

$\phi$

$\psi$

$\underline{\nabla}$

$\underline{x}$

$\mathrm{D} / \mathrm{Dt}$

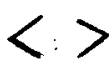

$+$

\section{$\underline{\text { Subscripts }}$}

i

$\mathrm{p}$

S

$\infty$

$\circ$

in

out

$I, I I, \ldots$

\section{Explanation and typical units}

stress tensor, $\mathrm{Kg} / \mathrm{m} \mathrm{sec}{ }^{2}$.

polar coordinate.

stream function.

del operator, /m.

vector cross product operator.

vector dot product operator.

differential operator as defined in text.

quantities enclosed are dimensionless.

(superscript) transpose of tensor.

value for component $i$.

value for the particle.

value at the particle surface.

value for large $r$.

value for large $r$ and $\theta=\pi / 2$.

(mean) inlet value.

(mean) outlet value.

values for various removal mechanisms. 


\section{REFERENCES}

Aitken, J., Trans. Roy. Soc. Edinb., 32, 239 (1883).

Annis, B. K., Malinauskas, A. P. and Mason, E. A., J. Aerosol Sci., 3, 55 (1972)

Annis, B. K., Malinauskas, A. P. and Mason, E. A., J. Aerosol Sci., 4 , 271 (1973).

Annis, B. K. and Mason, E. A., J. Aerosol Sci., 6, 105 (1975).

Azarniouch, M. K., Doctoral Thesis, Dept. of Chem. Eng.,

McGill University, Montreal (1974).

Azarniouch, M. K., Bobkowicz, A. J., Cooke, N. E. and Farkas, E. J., Can. J. Chem. Eng., 51, 590 (1973)

Azarniouch, M. K., Bobkowicz, A. J., Cooke, N. E. and Farkas, E. J., Can. J. Chem. Eng., 53, 278 (1975).

Bakanov, S. P. and Derjaguin, B. V., Disc. Faraday Soc., 30, 130 (1960).

Beal, S. K., Nucl. Sci. Eng., 40, 1 (1970).

Bird, R. B., Stewart, W. E. and Lightfoot, E. N., "Transport Phenomena", Wiley, N.Y., (1962).

Brock, J. R., J. Colloid Sci., 18, 489 (1963).

Brock, J. R., J. Colloid Interface Sci., 27, 95 (1968).

Byers, R. L. and Calvert, S., Ind. Eng. Chem. Fundam, 8, 646 (1969).

Calvert, S., Goldshmid, J., Leith, D. and Mehta, D., "Feasibility of Flux Force/Condensation Scrubbing for Fine Particle Collection" A.P.T. Inc., Riverside, Calif., N.T.I.S. \#PB-227-307, (1973).

Chapman, S. and Cowling, T. G., "The Mathematical Theory of NonUniform Gases", Cambridge University Press, London (1964). 
Dankwerts, P. V., "Gas-Liquid Reactions", McGraw-Hi11, N.Y. (1970). Davies, C. N. "Aerosol Science", Academic Press, N.Y. (1966). Derjaguin, B. V. and Bakanov, S. P., Dokl. Akad. Nauk SSSR, 117, 959. (1957).

Derjaguin, B. V. and Dukhin, S. S., Dokl. Akad. Nauk SSSR (Phys. Chem.) 106, 851; 111, 613 (1956).

Derjaguin, B. V. and Dukhin, S. S., Dok1. Akad. Nauk SSSR, 112, 407 (1957).

Derjaguin, B. V. and Yalamov, Yu. I., in "Topics in Current Aerosol Research", (Edited by Hidy, G. M. and Brock, J. R.), Vol. 3, Pergamon Press, Oxford (1972).

Derjaguin, B. V., Yalamov, Yu. I. and Storozhilova, A. I., J. Colloid Interface Sci., 22, 117. (1966).

Einstein, A., Z. Phys., 27, 1 (1924).

Facy, L., Comptes rendus, 246, 102; 246, 3161 (1958).

Fahnoe, F., Lindroos, A. E. and Abelson, R. J., Ind. Eng. Chem., 43, 1336 (1951).

Freise, V., J. Chem. Physique, 54, 879 (1957).

Friedlander, S. K. and Johnstone, H. F., Ind. Eng. Chem., 49, 1151 (1957).

Fuchs, N. and Kirsch, A., Chem. Eng. Sci., 20, 181 (1965).

Goldsmith, P., Delafield, H. J. and Cox, L. C., Geof. Pura. Applic., $\underline{50}, 278(1963)$.

Goldsmith, P., Delafield, H. J. and Cox, L. C., Q.J.R. Met Soc. 89, 43 (1963).

Goldsmith, P. and May, F. G., in "Aerosol Science" (Edited by Davies, C. N.), Academic Press, London (1966).

"Handbook of Physics and Chemistry" (Edited by Weast, R. C.), Chemical Rubber Co., Cleveland, Ohio (1970).

Happel, J. and Brenner,. H., "Low Reynolds Number Hydrodynamics", Prentice-Hall, New Jersey. (1965).

Hirschfelder, J. O., Curtiss, C. F. and Bird, R. B., "Molecular Theory of Gases and Liquids", Wiley, N.Y. (1964). 
Hutchinson, P., Hewitt, G. F. and Dukler, A. E., Chem. Eng. Sci., 26, 419 (1971)

International Business Machines Corp., "System/360 Continuous System Modelling Program Users' Manual", I.B.M., N.Y. (1969).

"International Critical Tables", McGraw-Hill, N.Y. (1926).

Kramers, H. A. and Kistemaker, J., Physica, 10, 699 (1943).

Lancaster, B. W. and Strauss, W., Ind. Eng . Chem. Fundam., 10, 362 (1971).

Lapple, C. E. and Kamack, H. J., Chem. Eng. Prog., 51, 110 (1955).

Le, C. D., "UBC SFRP Small Free-Format Regression Package", University of British Columbia Computing Centre, B.C. (1975).

Litvinov, A. T., Zh. Priklad. Khim., 40, 353 (1967).

Mason, E. A. and Chapman, S., J. Chem. Phys., 36, 627 (1962).

"Matheson Gas Data Book", 4 th Edn, The Matheson Co., New Jersey (1966).

Meisen, A., Doctoral Thesis, Dept. of Chem. Eng., McGill

University, Montreal (1970).

Meisen, A., Bobkowicz, A. J., Cooke, N. E. and Farkas, E. J., Can. J. Chem. Eng., 49,449 (1971).

Millikan, R. A., Phys. Rev., $\underline{32}, 382$ (1911).

Nishio, G., Kitani, S. and Takahashi, K., Ind. Eng. Chem., Process Des. Develop., 13, 408 (1974).

Perry, J. H. (Editor) "Chemical Engineers' Handbook", 4th edn. (1963).

Perry, J. H. and Chilton, C. H., (Editors), "Chemical Engineers" Handbook", 5th edn. (1973).

Prakash, C. B. and Murray, F. E., AIChE Symposium Series, 71, 81 (1975).

Rozen, A. M. and Kostin, V. M., Internat. Chem. Eng., 7, 464 (1967) .

Shaver, P. J., Ind. Eng. Chem., 43, 1532 (1951).

Schmitt, K. H., Staub, 21, 173 (1961). 
Schmitt, K. H. and Waldmann, L., Z. Naturf., 15a, 843 (1960).

Semrau, K. T.,Marynowski, C. W., Lunde, K. E. and Lapple, C. E., Ind. Eng. Chem. 50, 1615 (1958).

Sherwood, T. K. and Gilliland, E. R., Ind. Eng. Chem., 26, 516 (1934).

Sherwood, T. K. and Pigford, R. L., "Absorption and Extraction", 2nd edn., McGraw-Hill, N.Y. (1952).

Sparks, L. E. and Pilat, M. J., Atmos. Environ., 4, 651 (1970). Stefan, J., Wien. Ber., 83, 943 (1881)

Stinchcombe, R. A. and Goldsmith, P., J. Nuclear Energy, 20, 261 (1966).

Storozhilova, A. I., Dokl. Akad. Nauk SSSR, 155, 426 (1964).

Treybal, R. E., "Mass Transfer Operations", 2nd edn., McGraw-Hill, N.Y. (1968).

Truitt, J. and Davis, R. J., Amer. Ind. Hyg. Ass. J., 32, 583 (1971).

Waldmann, L., Z. Naturf., 14a, 589 (1959) .

Waldmann, L., in "Rarified Gas Dynamics", (Edited by Talbot, L.), Academic Press, N.Y. (1961).

Waldmann, L. and Schmitt, K. H., in "Aerosol Science", (Edited by Davies, C. N.), Academic Press, London (1966).

Wallis, G. B., "One Dimensional Two Phase Flow", McGraw-Hill, N.Y. (1969).

Whitmore, P. J. and Meisen, A., J. Aerosol Sci., 4, 435 (1973). Whitmore, P. J. and Meisen, A., J. Aerosol Sci., to be published. Whitmore, P. J. and Meisen, A., Can. J. Chem. Eng., to be published. 
Appendix A

\section{ROTAMETER CALIBRATION DATA}

The primary calibration curves for the liquid and gas rotameters are shown in figures A.1 to A.5. Secondary calibrations of the rotameters for other gases were made by scaling the primary calibrations, using the formula suggested by the manufacturer (Brooks). This formula is essentially the same as that given by Perry (1963).

$$
\frac{\mathrm{Q}_{\mathrm{A}}}{\mathrm{Q}_{\mathrm{B}}}=\sqrt{\frac{\rho_{\mathrm{B}}}{\rho_{\mathrm{A}}} \frac{\mathrm{T}_{\mathrm{B}}}{\mathrm{T}_{\mathrm{A}}} \frac{\mathrm{P}_{\mathrm{A}}}{\mathrm{P}_{\mathrm{B}}}}
$$

Here $Q, \rho, T$, and $P$ are the volumetric flow rate at standard conditions, density, temperature and pressure of gases $A$ and $B$. 


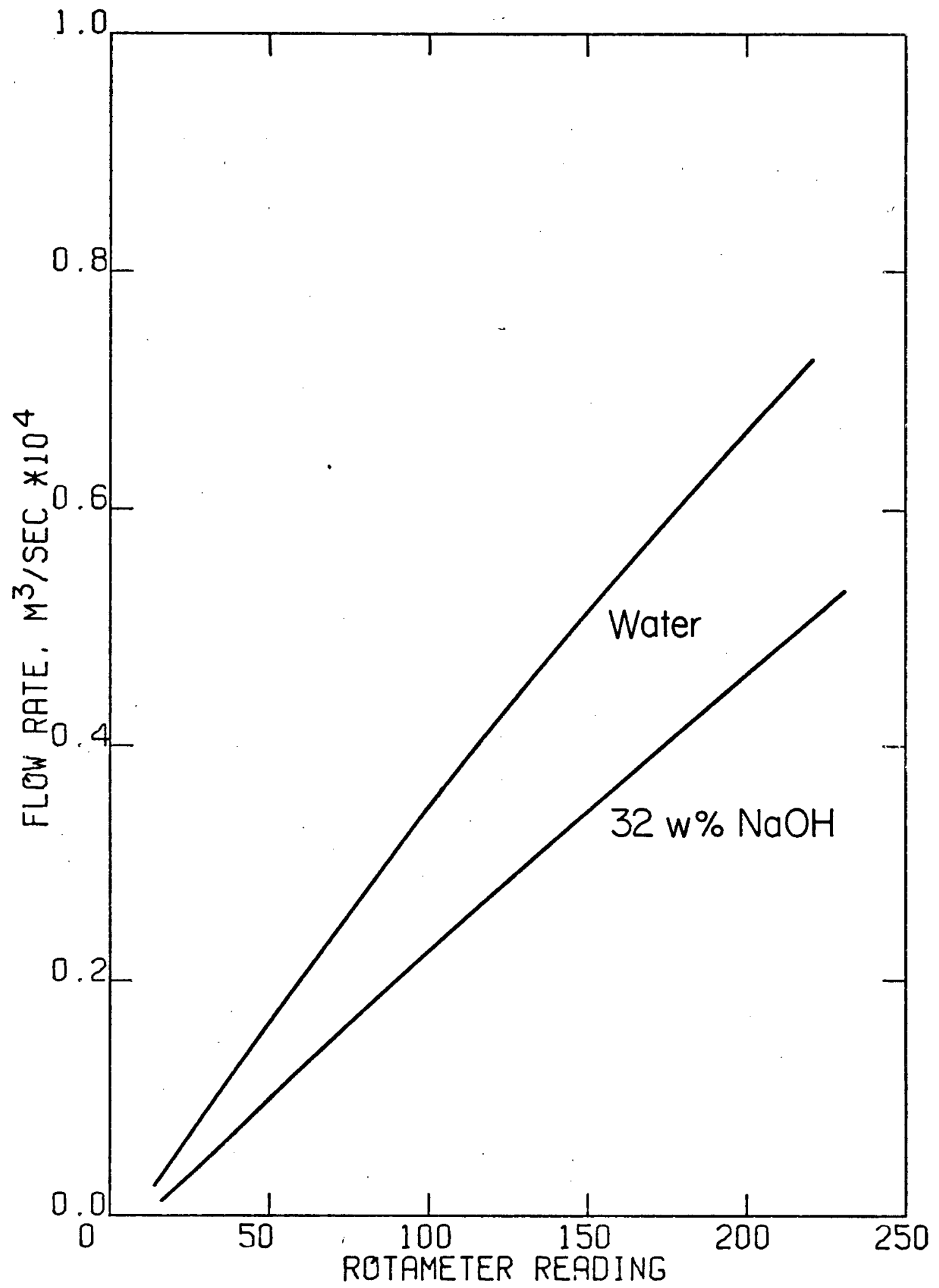

Figure A.1 Calibration curves for water and $32 \mathrm{w} \%$ caustic soda solution in the Brooks liquid rotameter. Liquid conditions $20^{\circ} \mathrm{C}$ and approximately 1 atmosphere. 
182 .

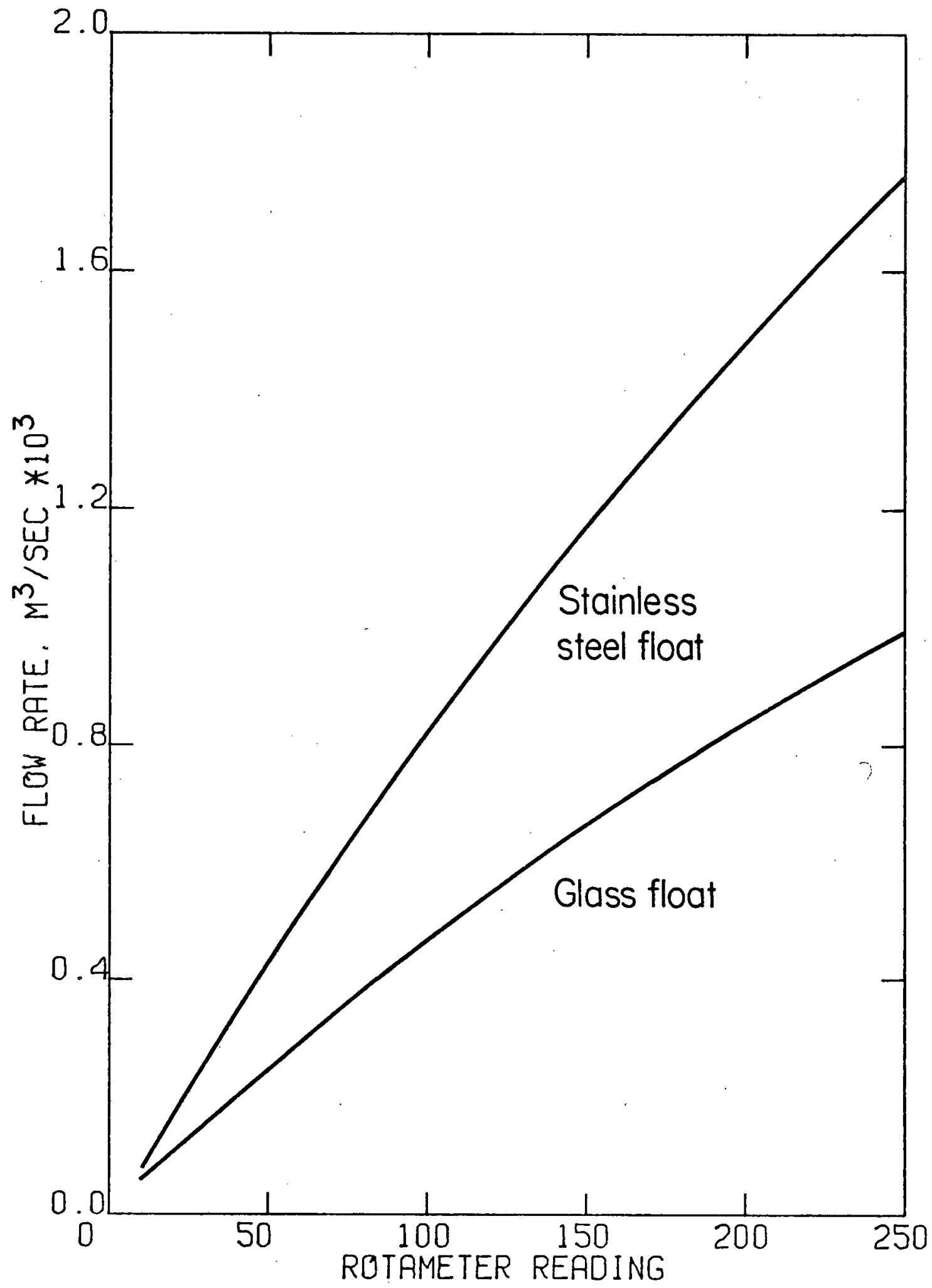

Figure A.2. Calibration curves for nitrogen in the Brooks inert gas rotameter. Gas condition - 4.40 atmospheres and $20^{\circ} \mathrm{C}$. 


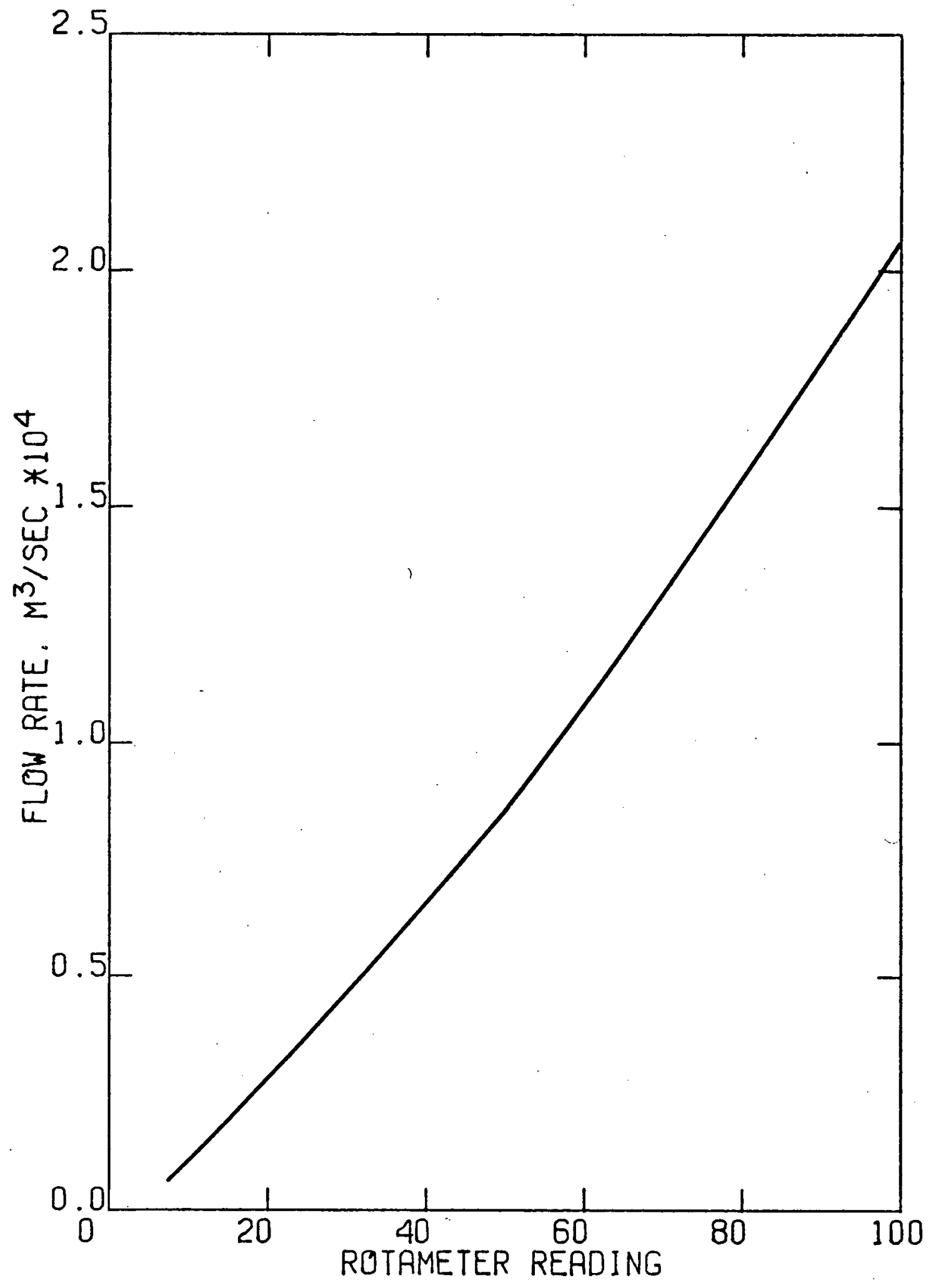

Figure A.3 Calibration curve for carbon dioxide in the size 3 Gilmont transferred gas rotameter. Gas conditions - 1 atmosphere and $20^{\circ} \mathrm{C}$. 


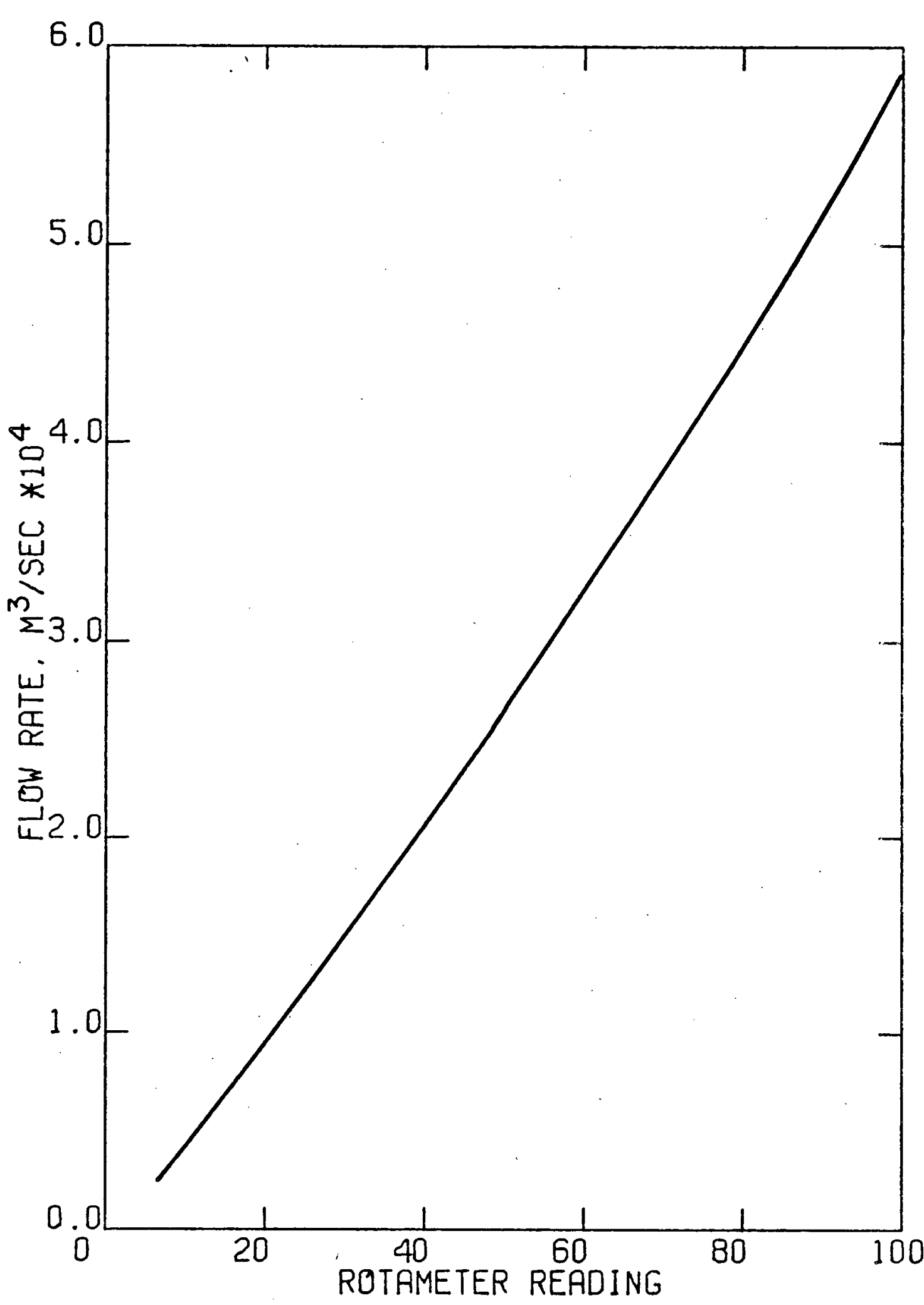

184. 


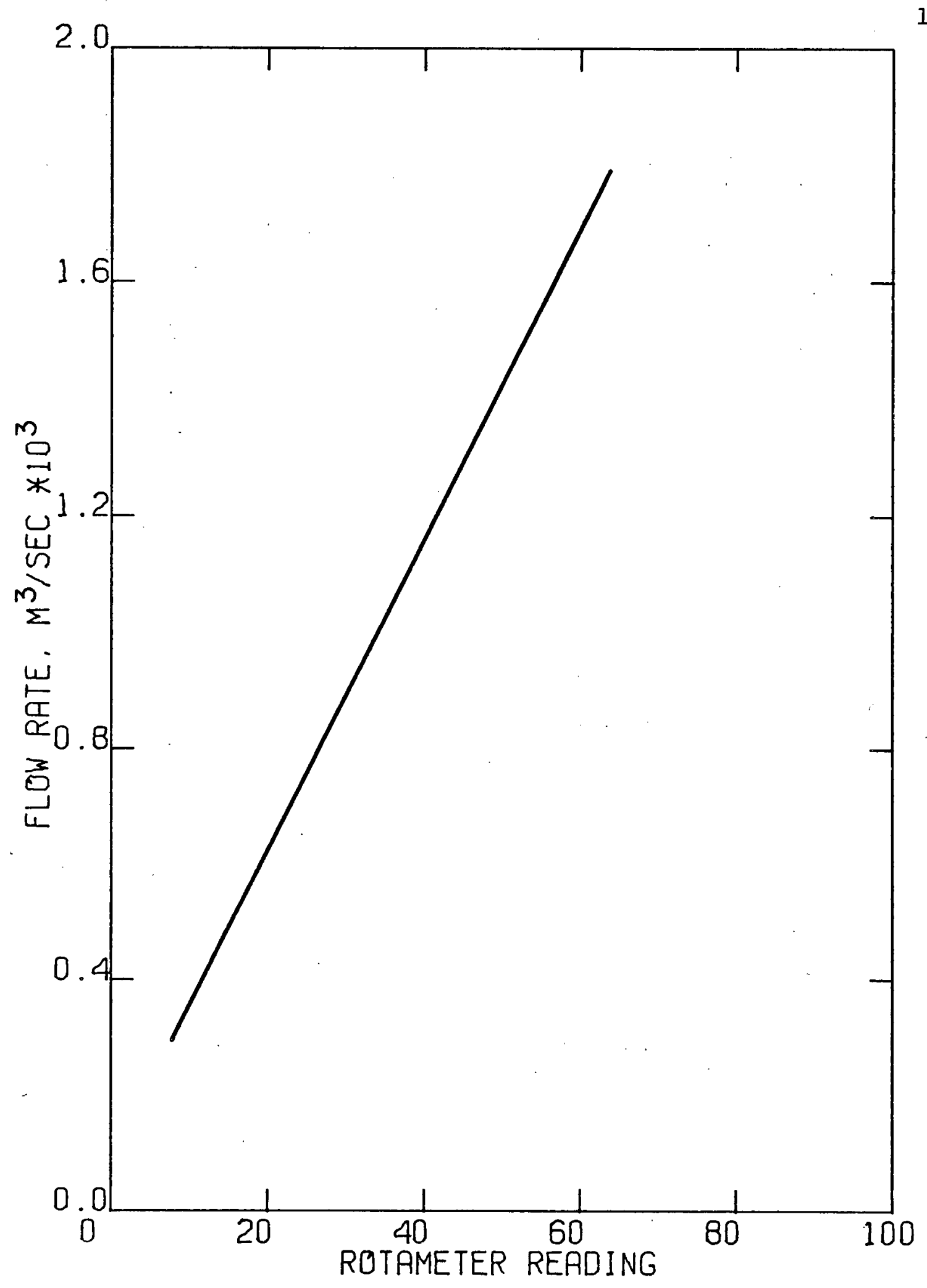

Figure A.5 Calibration curve for ammonia in the Brooks transferred gas rotameter.

Gas conditions - 1 atmosphere and $20^{\circ} \mathrm{C}$. 
Appendix B

\section{COLUMN MASS TRANSFER MODEL}

\section{B.1 Description}

The purpose of the model was to ascertain whether the liquid phase mass transfer resistance in the column was negligible compared to that of the gas phase. Since this condition was later shown to be unnecessary for the experiments, the modelling was abandoned after some preliminary work.

The model was constructed as follows. The gas phase mass transfer coefficient was assumed to be given by the correlation of Sherwood and Gilliland (1934), via the Sherwood number, Sh.

$$
\mathrm{Sh}=\mathrm{K} \mathrm{Re}^{0.83} \mathrm{Sc}^{0.44}
$$

However, the constant $\mathrm{K}$ was temporarily left undefined, and the Reynolds number was measured with respect to the liquid surface rather than to the surface of the dry column. The model also required a knowledge of the liquid surface velocity and film thickness profiles for the column. Information on the liquid film behaviour was taken 
from Bird, Stewart, and Lightfoot (1960), and Wallis (1969). The behaviour of laminar films under fully developed conditions is well understood, but in the present study the film accelerated after entering the column, and the bottom section appeared turbulent. The film Reynolds number is given by

$$
\operatorname{Re}_{\Gamma}=\frac{j_{L}{ }^{\rho}{ }_{L} D_{C}}{\mu_{L}}
$$

where $j_{L}$ is the volumetric flux based on the total column cross section, $D_{C}$ is the column diameter, and $\rho_{L}$ and $\mu_{L}$ are the liquid density and viscosity, respectively. The onset of turbulence is in the Reynolds number range of 1000 to 2000, while the Reynolds number under experimental conditions was 1760 in all cases, corresponding to a water flow of $35 \mathrm{ml} / \mathrm{sec}$. Under laminar conditions the surface velocity is 1.5 times the mean film velocity, and the equations given by Wallis (1969) imply that this is also a good approximation at low levels of turbulence. It was therefore assumed that this condition held at all times. The film acceleration was treated as follows. For constant flow one can calculate the accelerative field strength $b$, under which a film of given thickness would be stable. It was then assumed that the difference between this field strength and the actual field strength, $g$, was available to freely accelerate the liquid film surface. This approximation gives the correct terminal velocity. At the column inlet the liquid velocity must be greater than zero or the film thickness would be infinite. 
'The surface velocity. of the inlet liquid' was estimated by measuring the height of the liquid pool above the top of the column, and assuming the skin velocity was that attained by free fall through this distance. Typical values for this velocity were of the order of $0.3 \mathrm{~m} / \mathrm{s}$, which appeared realistic.

The model operated as follows. A value was assumed for the concentration of ammonia in the outlet gas. Calculations then started at the top of the column and proceeded downwards. The local mass transfer rate was found at each point from the local diameter, Reynolds number, and Schmidt number. The total mass transfer down to that point was obtained by integration. Thus the corresponding concentration of ammonia in the inlet gas was determined. If this was not in agreement with the specified inlet conditions, then a new outlet ammonia concentration was assumed and the calculations were repeated. Hence, eventually an outlet concentration was predicted which corresponded to the required inlet conditions.

\section{B. 2 Calculation and Results}

The model calculations were made using the computer simulation language CSMP (International Business Machines (1969)). Tests of the model were made using a typical set of experimental conditions for the ammonia-nitrogen system. The nitrogen flow rate was taken as $6 \times 10^{-4} \mathrm{~m}^{3} / \mathrm{sec}$, and the inlet mole fraction of ammonia was set at 0.450 . The constant in the formula for the gas phase mass transfer coefficient was then 
adjusted till the model predicted the same mole fraction of ammonia in the outlet gas as was measured experimentally. This adjustment was made for the usual water flow rate of $0.35 \times 10^{-6}$ $\mathrm{m}^{3} / \mathrm{sec}$. Experiments were also made using the same inlet gas rate and composition, but with a lower and a higher water flow rate. Similar results were obtained when these experiments were simulated by the model, as is shown in Figure B.l. The small difference between experimental measurements and model predictions may indicate a slight resistance in the liquid phase. Effects of surface rippling and liquid-induced gas turbulence were beyond the scope of this study. 


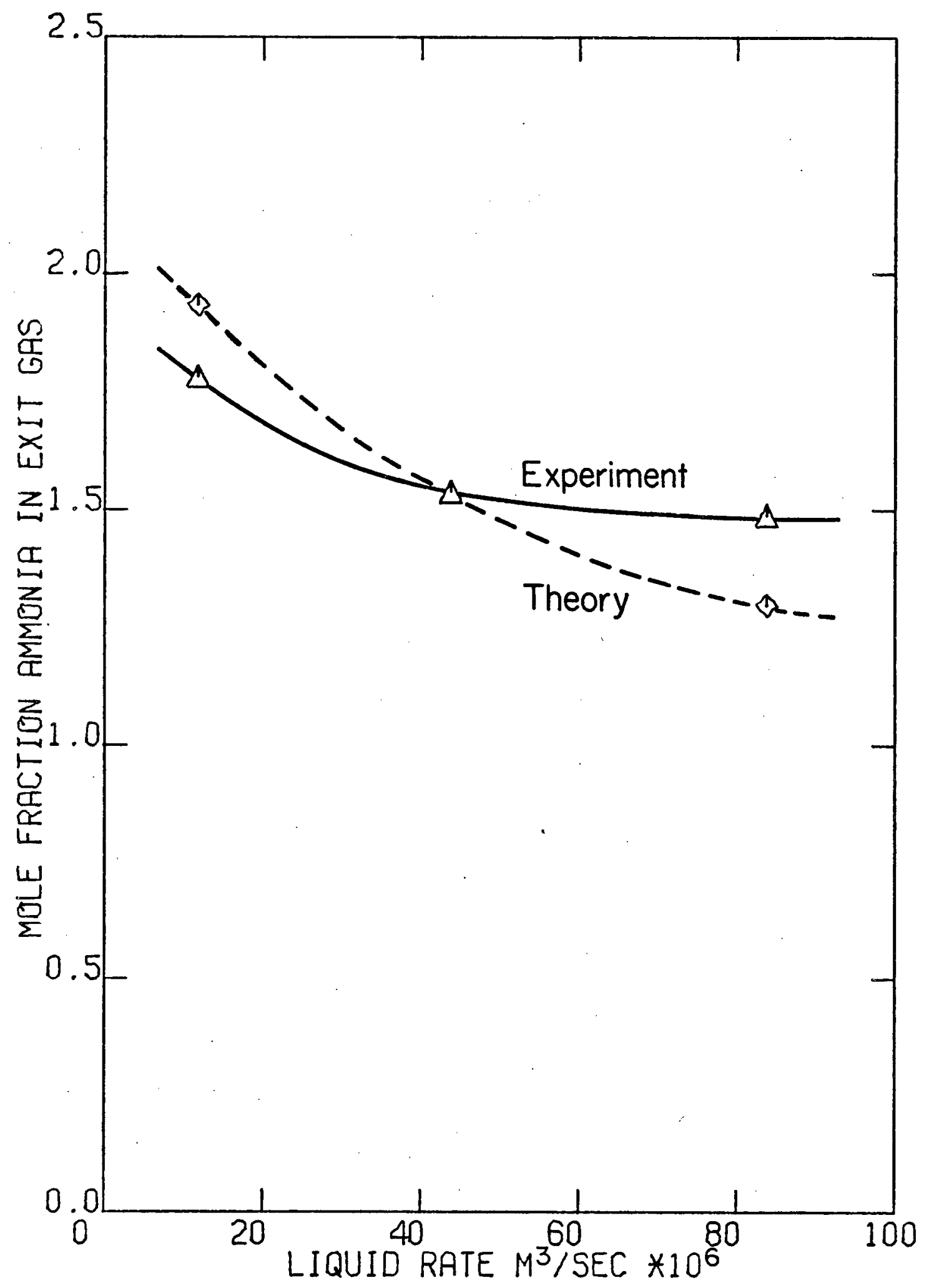

Figure B.1 Effect of liquid rate on outlet gas composition. 
Appendix C

\section{DATA ANALYSIS AND SAMPLE CALCULATIONS}

\section{C.1 Source of Sample Data}

The data used to illustrate the methods of analysis are taken from run 3501l, and are for $0.79 \mu \mathrm{m}$ particles with a nitrogen-ammonia gas mixture. Run 35011 lies in the mid-range of a series of runs forming an experiment in which the flow rate of the inert gas was held constant. The data for this run are. therefore typical, since they are for the most commonly used particle size, gas mixture, and type of experiment. The run also forms part of a series of runs in which the inlet gas composition was held constant at approximately 50 vo nitrogen, while the flow rate was varied. The results for this run can be found in Appendix D, Table XVI.

\section{C.2 Reduction of Raw Data}

Three results for fractional particle removal efficiency were calculated from the consecutive readings taken during run 35011. These appear in chronological order in Table XVI. 
The calculation of the second result is given here. Measurements of the particle count per unit volume of inert gas were made alternately at the top and base of the column, as described in Chapter 5, to yield the following data:

$\begin{array}{ccc}\text { Column top } & \text { Column base } & \text { Column top } \\ 5858 & 9058 & 6466 \\ 6345 & 8537 & 6243 \\ 6362 & 8604 & 5672 \\ 5808 & 9086 & 6120\end{array}$

These data did not contain any obviously spurious readings or exhibit any marked drift, and were therefore accepted and averaged.

Column top Column base
(bracketed average)

Mean

Variance

Standard deviation

Number of readings

$$
6109.25
$$

87113.13

295.15

8
8821.25

84736.00

291.09

4

The standard deviations of less than $5 \%$ of the means were quite satisfactory, and were comparable with those obtained in the other two sets of results derived from the run. The results were therefore accepted. The background counts taken at the top and base of the column with the particle generator turned off were negligible $(<5)$, and correction of the means calculated previously was therefore not necessary. The raw particle removal efficiency was then calculated. 


$$
\operatorname{EPEXP}=1-6109.25 / 8821.25=0.3074
$$

The variable names given are those used in the computer program and the data tables in Appendix D (see Table VIII).

\section{C.3 Counter Calibration for Gas Composition}

The efficiency, EPEXP, must be corrected for the influence of gas composition on particle count, as described in Chapter 5. Runs were therefore performed in which particles were added to a nitrogen stream to form an aerosol of constant concentration. This was then diluted with various proportions of ammonia. The readings with the ammonia present, and those with it absent, were averaged in a manner analogous to that used in calculating the particle removal efficiency. Thus, the following results were obtained for the ratio of counts with ammonia present to counts with ammonia absent, which is termed the calibration correction ratio, F.

$\begin{array}{llll}\text { Mole fraction of nitrogen } & 0.5040 & 0.6300 & 0.7600 \\ \text { when ammonia is present } & & & \\ \begin{array}{l}\text { Calibration correction } \\ \text { ratio }\end{array} & 0.9289 & 0.9732 & 0.9913 \\ & 0.9580 & 0.9607 & 0.9791 \\ & 0.9621 & 0.9969 & 0.9634 \\ & 0.9387 & 0.9720 & 0.9757 \\ & 0.9357 & 0.9512 & \\ & 0.9402 & & \\ & 0.9432 & & \\ & 0.9438 & 0.9708 & 0.9774 \\ \text { Mean value } & & & \end{array}$


These results are shown plotted in Figure C.l. Also, by definition, the correction ratio must be unity when the mole fraction of nitrogen, YINT, is one, so that the calibration curve must pass through this point. Since to a good approximation the mean values of the correction ratios lie along a straight line, the calibration correction can be expressed as a linear equation :

$$
F=0.8992+0.1008 \mathrm{YINT}
$$

\section{4 Calculation Methods \\ C. 4.1 General}

Calculation of the corrected particle removal efficiency, of the efficiencies given by the three models for the same experimental conditions, and of various other relevant parameters was done by means of the computer program given in Table IX. The nomenclature used in the program is explained in the key given in Table VIII. Since some of the variables used in the sample calculation are not referred to except in this appendix, it is convenient to use the program nomenclature.

\section{C.4.2 The Input Data}

The following experimental data are required to identify each experimental result and specify the experimental conditions. 


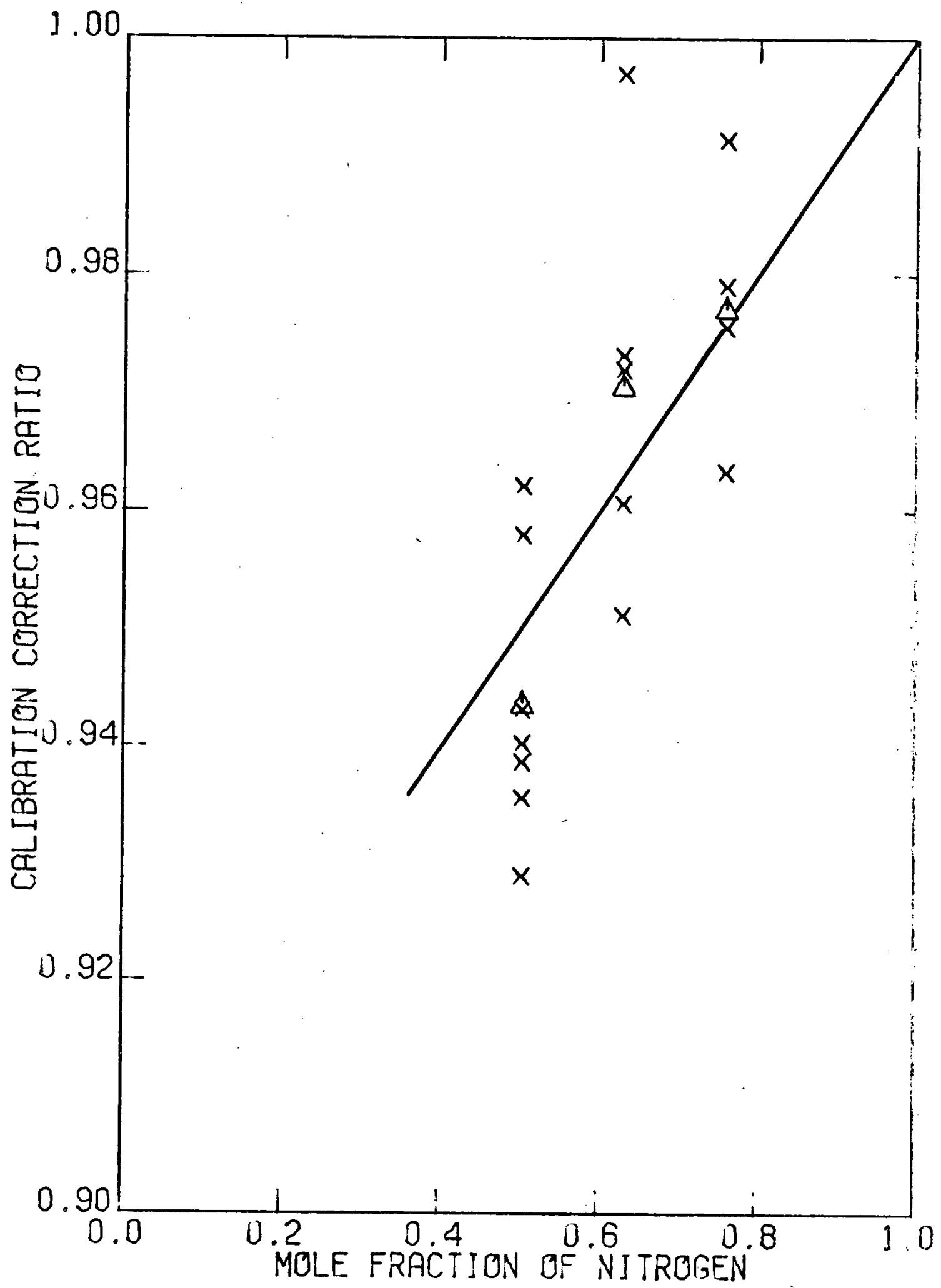

Figure C.1 Calibration correction ratio for nitrogen, ammonia, 0.79 micron diameter particles. The triangles denote mean values for a given mole fraction. 
Table VIII

Key to Calculation Program Nomenclature

Program
nomenclature

A

B

C

$\mathrm{D}$

DC

DIFF

$\mathrm{DM}$

DP

DPMU

DRIVE

EMO

EMT

EPCORR

EPEXP

EPMA

i

EPMO
Symbol in text

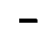

C

-

D

p

$-$

-

-

-

-

$\varepsilon_{\mathrm{p}}$

-

$\varepsilon_{\mathrm{MA}}$

$\varepsilon_{\mathrm{MO}}$
Explanation

Constant in equation for calibration correction ratio.

Constant in equation for calibration correction ratio.

Constant in equation for calibration correction ratio.

Molar density, $\mathrm{Kg}$ mole $/ \mathrm{m}^{3}$.

column diameter, m.

Diffusivity, $\mathrm{m}^{2} / \mathrm{sec}$.

Mass density $\mathrm{Kg} / \mathrm{m}^{3}$.

Particle diameter, m.

Particle diameter, m.

Log mean mass transfer driving force.

Fractional molar removal of total gas.

Fractional molar removal of transferred gas.

Corrected particle removal efficiency.

Experimental value for particle removal efficiency .

Efficiency prediction for mean mass velocity model.

Efficiency prediction for mean molar velocity model. 
Table VIII/cont'd

\begin{tabular}{|c|c|c|}
\hline $\begin{array}{l}\text { Program } \\
\text { nomenclature }\end{array}$ & $\begin{array}{l}\text { Symbol in } \\
\text { text }\end{array}$ & Explanation \\
\hline EPSW & $\varepsilon_{\text {SW }}$ & $\begin{array}{l}\text { Efficiency prediction for } \\
\text { Schmitt and Waldmann model. }\end{array}$ \\
\hline F & - & Calibration correction ratio. \\
\hline FILM & - & $\begin{array}{l}\text { Mass transfer film thickness, } \\
\text { m. }\end{array}$ \\
\hline FILMR & - & $\begin{array}{l}\text { Ratio of film thickness to } \\
\text { column radius. }\end{array}$ \\
\hline FLUX & $\mathrm{N}$ & $\begin{array}{l}\text { Mass transfer flux, } \mathrm{Kg} \text { mole/ } \\
\mathrm{m}^{2} \text { sec. }\end{array}$ \\
\hline FR & - & Experimental penetration. \\
\hline FRCORR & $\mathrm{P}$ & Corrected penetration. \\
\hline GAS & - & Chemical formula for gas. \\
\hline G & G & Gas molar flow rate $\mathrm{Kg}$ mole/sec. \\
\hline $\mathrm{KN}$ & $\mathrm{Kn}$ & Knudsen number. \\
\hline MTC & - & Mass transfer coefficient $\mathrm{m} / \mathrm{sec}$. \\
\hline $\mathrm{N}$ & - & Identification number for gas. \\
\hline NLINE & - & Line counter for paging. \\
\hline $\mathrm{P}$ & - & Mean free path, $\mathrm{m}$. \\
\hline $\mathrm{PR}$ & $\mathrm{P}$ & Pressure, atm. \\
\hline$Q$ & - & Volumetric flow rate, $\mathrm{m}^{3} / \mathrm{sec}$. \\
\hline RATE & - & Total mass transfer rate, $\mathrm{m}^{3} / \mathrm{sec}$. \\
\hline $\mathrm{RE}$ & $\operatorname{Re}$ & Reynolds number. \\
\hline RMA & - & $\begin{array}{l}\text { Ratio of inert gas to transferred } \\
\text { gas molecular weights. }\end{array}$ \\
\hline RSW & - & $\begin{array}{l}\text { Square root of ratio of inert gas } \\
\text { to transferred gas molecular weights. } \\
\text { continued ....... }\end{array}$ \\
\hline
\end{tabular}


Table VIII/cont'd

\begin{tabular}{|c|c|c|}
\hline $\begin{array}{l}\text { Program } \\
\text { nomenclature }\end{array}$ & $\begin{array}{l}\text { Symbol in } \\
\text { text }\end{array}$ & Explanation \\
\hline RUN & - & Run identification number. \\
\hline SQ & - & $\begin{array}{l}\text { Square root of molecular weight, } \\
(\mathrm{g} / \mathrm{g} \text { mole })^{\frac{1}{2}} \text {. }\end{array}$ \\
\hline $\mathrm{T}$ & - & Temperature, ${ }^{\circ} \mathrm{K}$. \\
\hline TYPE & - & Type of experiment. \\
\hline $\mathrm{U}$ & - & Mean axial velocity, $\mathrm{m} / \mathrm{sec}$. \\
\hline UR & - & $\begin{array}{l}\text { Ratio of radial velocity in film } \\
\text { to mean axial velocity. }\end{array}$ \\
\hline URAD & - & Radial velocity in film, $\mathrm{m} / \mathrm{sec}$. \\
\hline $\mathrm{V}$ & $\mu$ & Viscosity, $\mathrm{Kg} / \mathrm{m}$ sec. \\
\hline VERBAL & - & $\begin{array}{l}\text { Verbal message concerning data } \\
\text { in table. }\end{array}$ \\
\hline V3 & - & $\begin{array}{l}\text { Cube root of molecular volume, } \\
(\mathrm{m} / \mathrm{g} \text { mole }) 1 / 3 \text {. }\end{array}$ \\
\hline W & M & Molecular weight, $\mathrm{g} / \mathrm{g}$ mole. \\
\hline $\mathrm{Y}$ & $\gamma$ & Mole fraction. \\
\hline $\mathrm{zC}$ & - & Column length, $\mathrm{m}$. \\
\hline
\end{tabular}

Postscripts

-INT

Value for inert gas.

-TRN

Value for transferred gas.

-MIX

Value for gas mixture.

$-I$

Value at gas inlet.

$-0$

Value at gas outlet.

- LM

Value where the mass transfer driving force equals the log mean ariving force over the column. 
Listing of Calculation Program

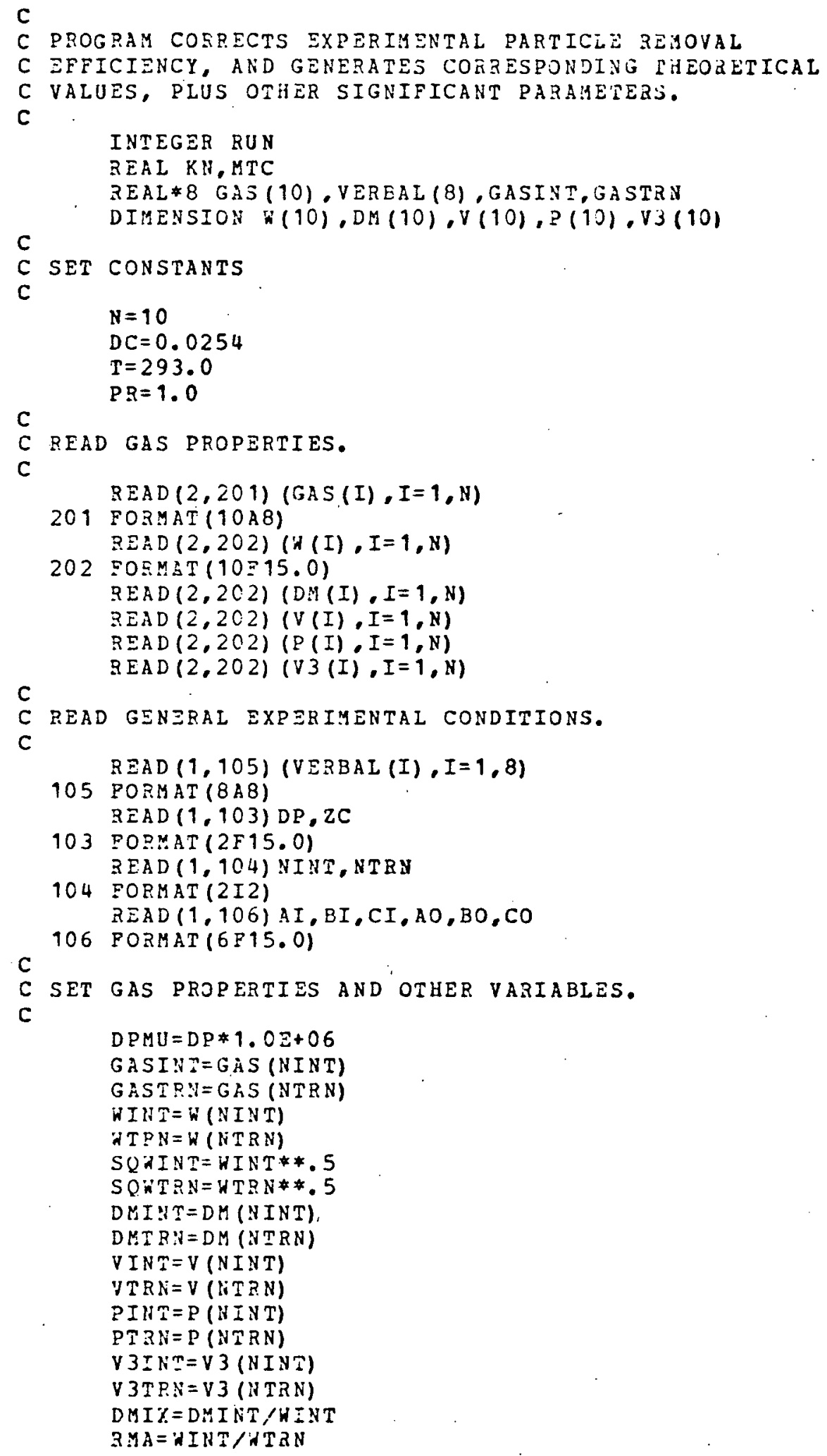




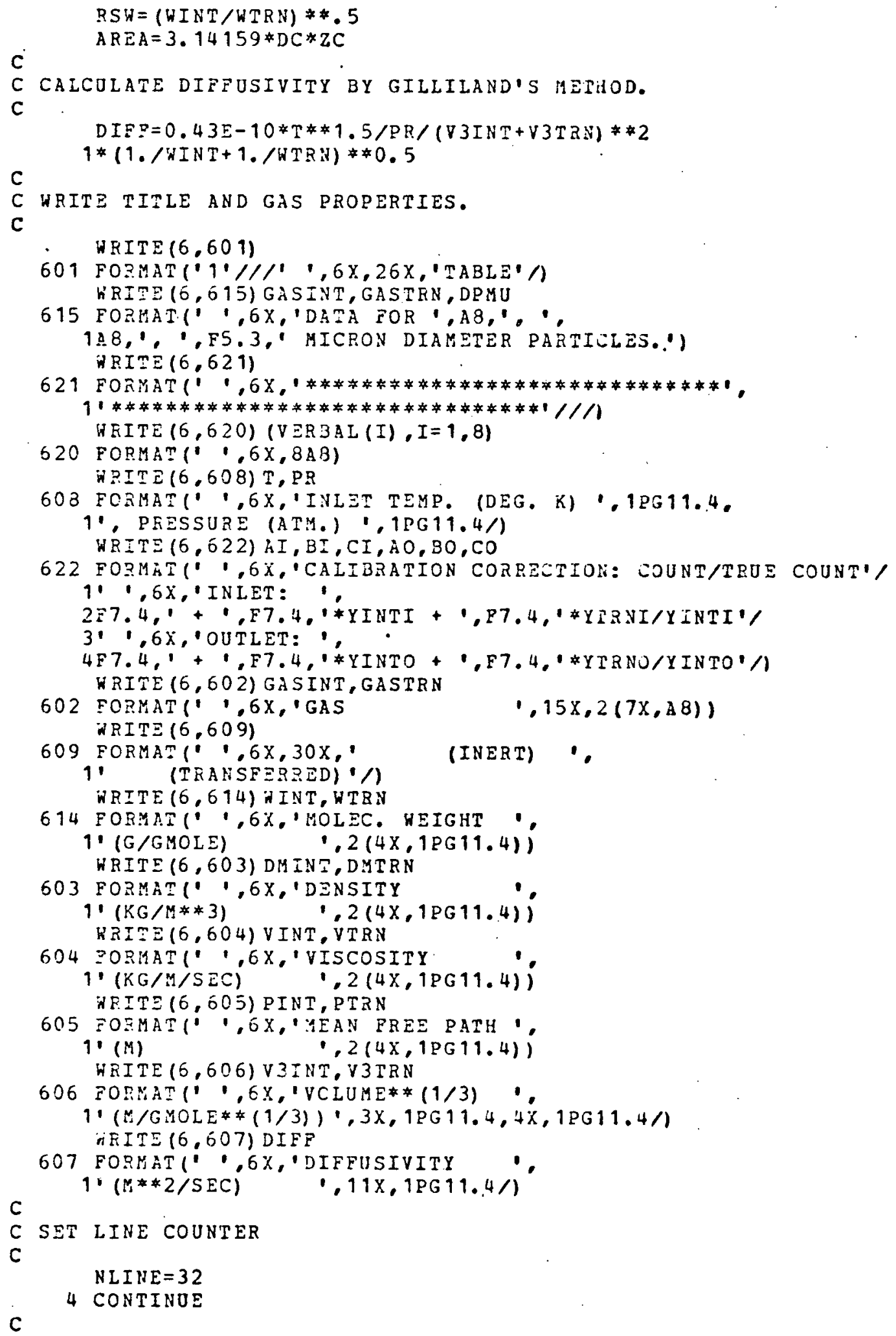




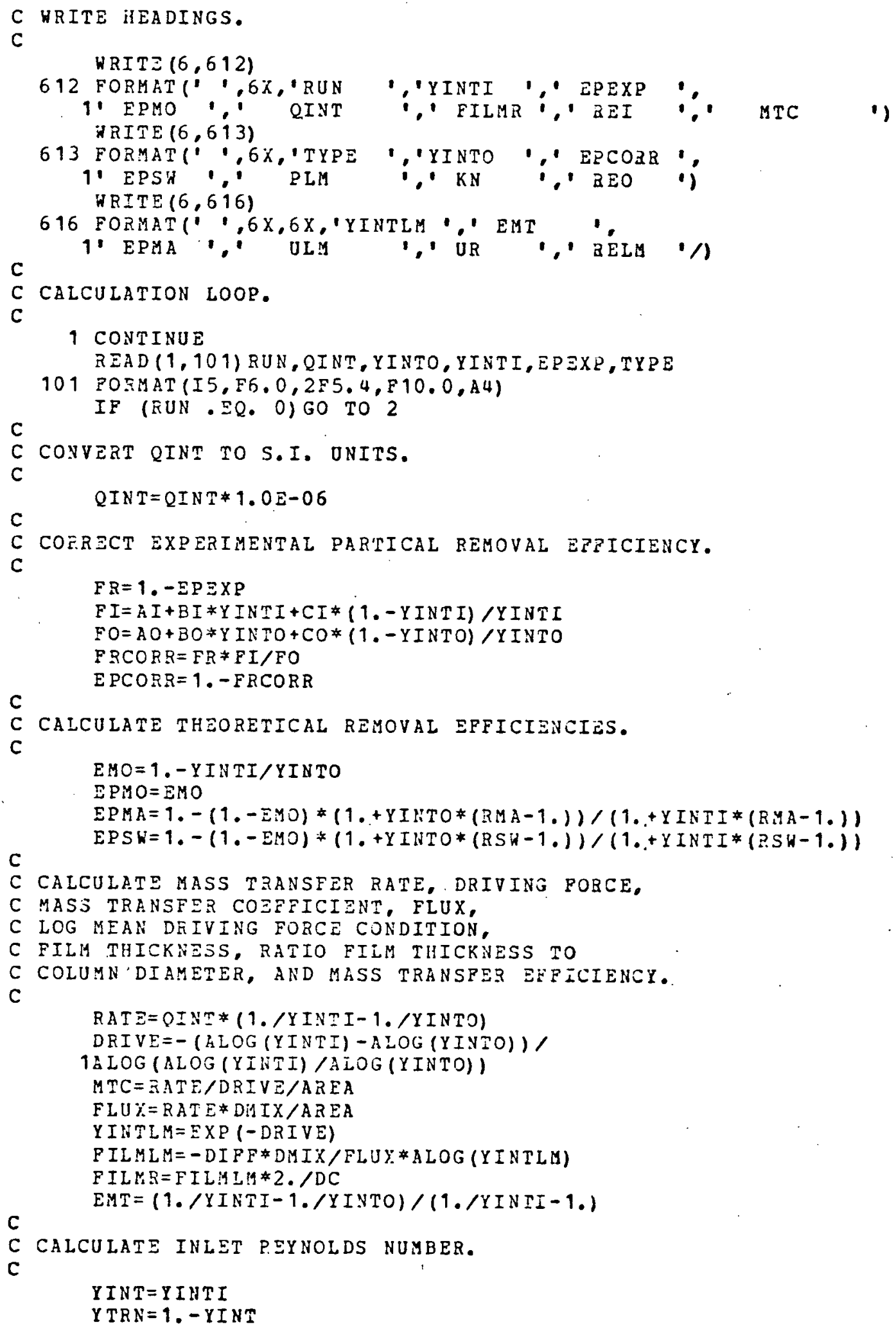


GINT $=Q I N T * D M I N T / H I N T$

GMIX $=G I N T / Y I N T$

WMIX = WINT*YINT+4TRH*YTRN

VMIX $=(Y T R A * S Q W T R N * V T R N+Y I N T * S Q N I N T * V I N T) /$

1 (YTRN*SQNTRN+YIVT*SQNINT)

$U=G M I X / D M I X * 1.2732 / D C / D C$

C $R E I=D C * D M I X * U / V M I X * M M I X$

C

C

YINT $=$ YINTO

YTRN $=1 .-Y I N T$

GINT $=$ QINT*DNINT/UINT

GMIY $=G I N T / Y I N T$

NYIX $=W I N T * Y I N T+H T R N * Y T R N$

VMIX=(YTRN*SQHTRH*VTSN+YINT*SQHINT*VINT)

1 (YTE:N*SQWTEN+YINT*SQWINT)

$U=G M I X / D K I X * 1.2732 / D C / D C$

$R E O=D C * D M I X * U / V M I X * W M I X$

C

C CALCULATE REYNOLDS WUMBER AT LOG MEAN CONDITION.

c

$Y I N T=Y I N T L M$

$Y T K N=1 .-Y I N T$

$G I N T=Q I N T * D M I N T /$ TNT

$G M I X=G I N I / Y I N T$

WMIX $=$ XIINT Y Y INT + TTRN

VMIX $=(Y T R R * S 2 A T R N * V T R N+Y I N T * S Q$ TIIT*VINT)

$1(X T R N * S O N T R N+Y I N T$ SQ TINT)

$\tilde{J}=G Y I X / D A I \dddot{X} * 1.2732 / D C / D C$

$R E I M=D C * D M I X * U / V Y I X * * M I X$ $\mathrm{ULM}=\mathrm{U}$

c

C CALCULATE RATIO OP RADIAL VELOCITY AT EDSE OF FILA

C TO MEAN AXIAL VELOCITY AT LOG YEAN CONDITION.

C

URAD = WIRN*FLUX/WYIX/DHIX

$U R=U R A D / U$

c

C CALCULATE FAIIO OP GAS MEAN FREE PATH AT

C LOG AEAN CONDITION TO PARTICIE RADIUS.

C

PLM $=$ VMIX/DMIX/WHIX/

1 (YINT*VINT/DMINT/PINT+YTRN*VTRN/DHTRN/RTRN) $K N=P L: 1 * 2 . / D P$

C

C WRITE RESULTS.

HRITE $(6,610)$ RUN, YINTI, EPEXP, EPYO, QINI, FILUE,REI, ATC

610 FORMAT!' $, 6 X, I 5,1 X, F 5,4,1 X, F 7,4,1 X, k 6.4,1 X$,

$11 \mathrm{PG} 10.4,1 X, 0 \mathrm{PE} 6.4,1 \mathrm{X}, \mathrm{F5}, 0,1 \mathrm{X}, 1 \mathrm{PG} 10.41$

WRITE $(0,611)$ TYPE, YINTO, EPCOBR, EPSW, PLH,KN, REO

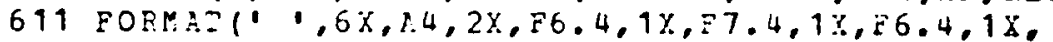

$11 \mathrm{PG} 10.4,1 \mathrm{X}, 0 \mathrm{PE} 0.4,1 \mathrm{X}, \mathrm{F} 6.0,1 \mathrm{X})$

WRITE $(6,617)$ YINTLA, EMT, EPMA,ULN,UR,RELM

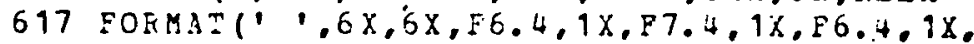

C 
Run number (RUN)

Type of experiment (TYPE)

Inert gas volumetric flow rate (QINT)

Inert gas mole fraction at inlet. (YINTI)

Inert gas mole fraction at outlet (YINTO)

Recorded experimental efficiency (EPEXP)
35011

IC

$6 \times 10^{-4} \mathrm{~m}^{3} / \mathrm{sec}$

0.5040

0.7860

0.3074

The value of the variable TYPE indicates the sort of experiment in which the data were taken. Values used are:

I Flow rate of inert gas held constant.

T Inlet flow rate of transferred gas held constant.

$\mathrm{C}$

Inlet gas composition held constant.

I2,I3 Flow rate of inert gas held constant at a different value than that used in the other experiment(s).

Combinations The data belong to more than one type of letters of experiment.

Other general data is required for the whole series of experiments.

(i) Dimensional data.

$$
\begin{array}{ll}
\text { Particle diameter (DC) } & 0.79 \mu \mathrm{m} \\
\text { Column length (ZC) } & 0.77 \mu \mathrm{m}
\end{array}
$$

(ii) Physical properties of the gases used.

These properties, which are shown in Table $\mathrm{x}$ together

with their sources, must be known in order to calculate the theoretical particle removal efficiencies, and the physical properties of the gas mixture.

(iii) The counter calibration correction.

It was found that the calibration correction ratio 
Table $\mathrm{X}$

Physical Properties of Gases at 1 atm. and $20^{\circ} \mathrm{C}$.

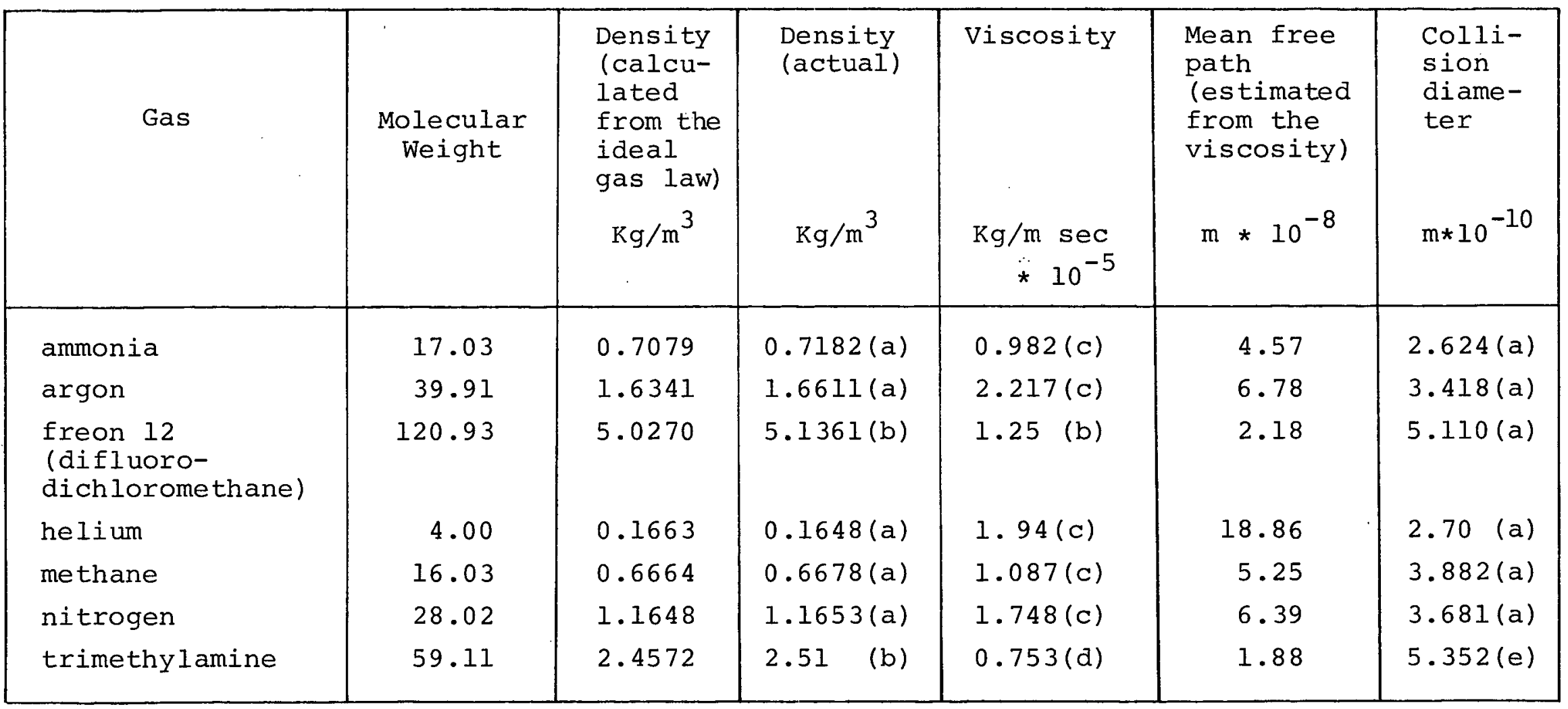

Source : (a) Perry (1963)

(b) Matheson data book (1966)

(c) Handbook of Physics and Chemistry (1970)

(d) Estimated by Bromley-Wilke method, see Perry (1963)

(e) Estimated from molal volume data, see Perry (1963) 
could always be expressed as a linear function of gas composition, in one of two forms. This function is shown in each table in Appendix D, and varies with both gas system and particle size. Where the inlet counts were made with the transferred gas absent, only an outlet correction is shown: Using the calibration correction function, the corrected particle removal efficiency (EPCORR) can be calculated from the experimental value (EPEXP).

\section{4.3 Calculation of Gas Mixture Properties}

Physical properties of the gas mixture must be determined as intermediate step in the calculation of other parameters such as Knudsen and Reynolds numbers. The following calculation identities were used.

(i) Molecular weight.

$$
\text { WMIX }=\text { WINT * YINT + WTRN * YTRN }
$$

(ii) Density .

$$
\text { DMMIX }=\text { DMIX *WMIX }
$$

\section{(iii) Viscosity.}

$$
\operatorname{VMIX}=\frac{\text { YTRN } * \text { VTRN } * \sqrt{\text { WTRN }}+\text { YINT } * \text { VINT } * \sqrt{\text { WINT }}}{\text { YTRN } * \sqrt{\text { WTRN }}+\text { YINT } * \sqrt{\text { WINT }}}
$$

The formula is taken from Perry (1963), and is normally accurate to within $2 \%$. 
(iv) Diffusivity.

$$
\mathrm{DIFF}=4.3 * 10^{-10} \frac{\mathrm{T}^{1.5}}{\operatorname{PR}\left[\left(\mathrm{VINT}^{1 / 3}+(\mathrm{VTRN})^{1 / 3}\right]\right.} \sqrt{\frac{1}{\mathrm{MINT}}+\frac{1}{\mathrm{MTRN}}}
$$

This relation was suggested by Gilliland, and is given by Perry (1963) .

(v) Mean free path.

$$
P=\frac{\text { VMIX }}{\text { DMMIX }} \frac{\mathrm{L}}{\frac{\text { YINT *VINT }}{\text { DMINT * PINT }}+\frac{\text { YTRN *VTRN }}{\text { DMTRN * PTRN }}}
$$

The formula for the gas mean free path, $\mathrm{L}_{\text {mix }}$, was derived from an approximation relating it to the mixture viscosity, " ${ }_{\text {mix }}$ ' the density, $\rho_{\text {mix }}$ ' and the mean molecular velocity in the mixture $\overline{\mathrm{c}}_{\operatorname{mix}}$.

$$
\mu_{\operatorname{mix}}=0.499 \rho_{\operatorname{mix}} \overline{\mathrm{C}}_{\operatorname{mix}} I_{\operatorname{mix}}
$$

Here, the mean molecular velocity is obtained from the relation

$$
\bar{c}_{\operatorname{mix}}=\gamma_{1} \bar{c}_{1}+\gamma_{2} \overline{\bar{c}}_{2},
$$

where $\bar{c}_{1}$ and $\bar{c}_{2}$ are the component molecular velocities. The relationship is analogous to that for a pure gas (see for example, Chapman and Cowling (1964)).

$$
\mu=0.499 \rho \overline{\mathrm{C}} \mathrm{L} .
$$




\section{C.4.4 Calculation of the Particle Removal Efficiency}

The corrected efficiency is obtained from the raw efficiency, using the formula

$$
\text { EPCORR }=1-(1-\text { EPEXP }) \text { FI/FO }
$$

where FI and FO are the inlet and outlet correction ratios. Run 35011 was made using the technique where the bottom reading is taken with the transferred gas shut off. This can be deduced from Table XVI, since no calibration correction is indicated at the gas inlet. The true top count is slightly greater than the recorded count, so that the true removal efficiency is slightly lower than the uncorrected value.

$$
\text { EPCORR }=1-(1-0.3074) 1 / 0.9784=0.2921
$$

\section{C.4.5 Calculation of the Theoretical Values for Particle Removal Efficiency}

Three theoretical values for the removal efficiency were calculated, based on the measured inlet and outlet inert gas concentrations (YINTI and YINTO), and the gas component molecular weights. These were derived from the three models given in Chapter 4, in which the particle was assumed to move with the local mean mass velocity of the fluid (EPMA), the local mean molar velocity (EPMO), or the velocity suggested by Schmitt and Walmann (EPSW). 


\section{C.4.6 Calculation of Other Parameters}

Most of these parameters were calculated because their values indicate whether a divergence might be expected between the data and the mean mass velocity model predictions. With the exception of the inlet and outlet Reynolds numbers (REI and REO), the parameters were calculated for a mean gas composition, which was representative of the mean conditions in the column. Since the total mass transfer was not a linear function of distance from the base of the column, a simple average of inlet and outlet concentrations did not yield a satisfactory mean. Instead, the mean was taken to occur at the position where the local mass transfer driving force (assuming negligible liquid phase resistance) equaled the log mean driving force over the column. The concentration at this point for the inert gas, YINTLM, is given by the following equations.

$$
\begin{aligned}
\text { DRIVE } & =-\frac{[\ln (\text { YINTI })-\ln (\text { YINTO })]}{\ln [\ln (\text { YINTI }) / \ln (\text { YINTO })]} \\
\text { YINTLM } & =\operatorname{EXP}(- \text { DRIVE })
\end{aligned}
$$

Four major parameters were calculated at this mean concentration.

(i) The Reynolds number (RELM).

Any particle losses, other than by diffusiophoresis, were probably attributable to inertial deposition. For a given particle size, these losses should increase as the Reynolds 
number increases.

(ii) The Knudsen number (KN).

The onset of transition behaviour is dependent on the value of the Knudsen number. As this number increases, increasing deviations from large particle behaviour can be expected.

(iii) The ratio of film thickness to column radius (FILMR) .

When this ratio becomes significant, the film theory of particle deposition no longer holds. However, in the more general theory the film thickness has no influence, and the value of the ratio was therefore not expected to be important. Statistical tests on the parameter will not yield useful information because of its very close correlation with Reynolds number. The ratio was calculated using elementary mass transfer film theory (see Treybal (1968)).

(iv) The ratio of gas radial velocity in the film to mean axial velocity (UR).

As in the previous case, this ratio relates to possible breakdown of the film model, but in this instance it is due to imperfect mixing in the gas core at very high mass transfer rates. Again, the more general theory indicates that the parameter is not important, and the close correlation with Reynolds number makes statistical testing impossible. The ratio was calculated using the radial gas mean mass velocity at the edge of the film adjacent to the turbulent core, which can be determined from elementary film theory (see Treybal (1968)).

Other parameters calculated only for their general interest were the mass transfer coefficient (MTC), and the mass 
transfer efficiency expressed in terms of the fractional molar removal of the transferred gas (EMT).

\section{C.4.7 The Computed Results}

The output of the program is the tabulated data in Appendix D. For each experimental reading the following information is given.

Run number (RUN)

Type of experiment (TYPE)

Inert gas volumetric flow rate (QINT)

Inert gas mole fraction at inlet (YINTI) 0.5040

Inert gas mole fraction at outlet (YINTO) 0.7860

Recorded experimental efficiency (EPEXP)

Corrected experimental efficiency (EPCORR) 0.2921

Mean mass velocity model prediction (EPMA)

Mean molar velocity model prediction (EPMO)

Schmitt and Waldmann velocity model

prediction (EPSW)

Inert gas mole fraction at the mean concentration (YINTLM)

Ratio of film thickness to column radius at the mean concentration (FILMR)

Gas mean free path at the mean concentration (PLM)

$5.8877 \times 10^{-8} \mathrm{~m}$

Knudsen number at the mean concentration (KN)
0.3140

0.6538

0.2707

0.3588

1117

0.1491 
Mean axial velocity at the mean concentration (ULM)

Ratio of radial velocity in the film to mean axial velocity at the mean concentration (UR)

Reynolds number at the mean concentration (RELM)

Reynolds. number at inlet (REI)

Reynolds number at outlet (REO)

Mass transfer coefficient (MTC)

Mass transfer efficiency (EMT)

In addition, the physical properties of the gas
$1.811 \mathrm{~m} / \mathrm{s}$

0.0027

3039

3957

2531

$1.6358 \times 10^{-2}$

0.7322

components are given, as are the calibration corrections used for the inlet and outlet gas.

\section{C.5 Error Analysis}

The magnitude of possible errors, and their influence on the experimental removal efficiencies and theoretical model predictions, are considered in this section.

(i) Random scatter in the aerosol counts.

The scatter arose from random fluctuations in aerosol concentration, and from experimental errors in determining the time for a given volume of gas to pass through the gas meter. The latter could have been caused by errors in the gas meter or by timing errors. Since the gas meter was claimed to have an absolute accuracy of $\pm \frac{1}{2} \%$, and only relative accuracy between readings was necessary, the possible error from this source was expected to be small. The timing error was estimated as \pm 1 . . Neither of 
these errors will be systematic.

Random errors are best treated by statistical methods (see Section C.6).

(ii) The Calibration correction.

The possible error in the calibration correction ratio is indicated by the scatter in the data used to establish the correction curve. This error increased as the mole fraction of transferred gas increased. However, for mathematical reasons, a given fractional error in the correction ratio leads to an absolute error in the measured particle removal efficiency which is largest at low efficiencies. Hence the magnitude of the error depends in a complex way on gas composition. The values quoted in Table IV are typical values for intermediate experimental conditions.

The possible error in run 35011 can be calculäted as follows. Since the bottom reading was taken with the ammonia absent, the correction only applies to the top reading. The mole fraction of nitrogen at the outlet was 0.7860 . Figure C.1 indicates that the possible percentage error in the correction ratio for this mole fraction is $\sim \pm 1.5 \%$. The absolute error in the efficiency can then be calculated

$$
\begin{aligned}
\text { EPCORR } & =1-0.6926 \times 1 /(0.9748 \pm 1.5 \%) \\
& =1-(0.7079 \pm 1.5 \%) \\
& =0.292 \pm 0.011
\end{aligned}
$$

(iii) Errors in the theoretical model predictions. These errors may have been caused either by errors in 
the gas analyses used to specify experimental conditions, or by errors which were due to extraneous species being present in the gas. These species would arise mainly as impurities in the gases as supplied.

The effect of possible gas analysis errors for run 35011 is now calculated.

$$
\begin{aligned}
\text { EPMA } & =1-\frac{[(0.7860 \pm 0.004) 28.02+(0.2140 \pm 0.004) 17.03] /(0.7860 \pm 0.004)}{[(0.5040 \pm 0.004) 28.02+(0.4960 \pm 0.004) 17.03] /(0.504 \pm 0.004)} \\
& =1-\frac{(25.66 \pm 0.05) /(0.7860 \pm 0.004)}{(22.56 \pm 0.05) /(0.5040 \pm 0.004)} \\
& =1-\frac{(25.66 \pm 0.19 \%) /(0.7860 \pm 0.51 \%)}{(22.56 \pm 0.22 \%) /(0.5040 \pm 0.79 \%)} \\
& =1-\frac{(32.64 \pm 0.32 \%)}{(44.76 \pm 0.57 \%)} \\
& =1-(0.729 \pm 0.89 \%) \\
& =0.271 \pm 0.006
\end{aligned}
$$

Since the errors in the terms in the numerator are interrelated, the signs must be taken into account when they are combined. The same applies to the denominator errors. The analysis indicates that, in general, the absolute error in the efficiency will be of the order of twice the absolute error in the gas analysis. The 
gas analysis error was estimated as \pm 0.004 except for the freon 12 results where it was taken as \pm 0.008 because of the difficulties in analysing very high inert fractions, and the very slight solubility of freon 12 in the absorbing solution.

The error in the mean mass velocity model due to the presence of extraneous gas species was estimated as follows. Suppose the fractional impurity levels in the inert and transferred gases as supplied are $\mathrm{x}$ and $\mathrm{y}$, respectively. Assume that these impurities can have a molecular weight of from $\frac{1}{2}$ to $1 \frac{1}{2}$ times the molecular weight of the pure component. Assume for the inert gas typical inlet and outlet mole fractions of 0.5 and 0.75 . The error can be considered as arising from the molecular weight only. The inlet molecular weight is

$$
0.5\left(\text { WINT } \pm x \frac{\text { WINT }}{2}\right)+0.5\left(\text { WTRN } \pm y \frac{W T R N}{2}\right)
$$

If the molecular weights of the inert and transferred species are of the same order, then the fractional error in the: inlet molecular weight is given by $\pm(x+y) / 4$. Similarly, the fractional error in the outlet molecular weight is $\pm(3 x / 8+y / 8)$. Since the signs of these errors are related for each gas, the fractional error in the penetration is given by the difference, $\pm(x+y) / 8$. This will translate into an absolute error in the particle removal efficiency of up to $\pm(x+y) / 8$. Errors of the same order will arise in the other two model predictions.

\section{(iv) other errors.}

It was not possible to quantify the errors arising from 
other sources such as inertial deposition, isokinetic effects, thermophoretic influences, and particle generation in the column. However, it was believed that these could not introduce an absolute error of more than approximately \pm 0.015 in the fractional particle removal efficiency during normal operation, and the probable error was expected to be negligible. 
Appendix D

\section{TABULATED RESULTS}

All experimental results, the conditions under which they were obtained, and various associated parameters such as predicted particle removal efficiencies, are listed in the following tables. A complete explanation of notation used and the relevance of the variables appears in Appendix $\mathrm{C}$. 
TABLEXI

DATA FOR HE NH3, N.790 MICRON DIAMETER PARTICLES。

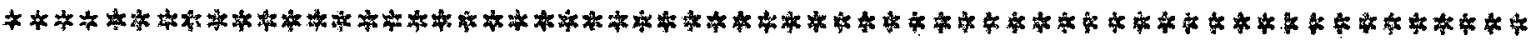

TRANSFERRED GAS FLJW RATE CONSTANT EXCEPT IV RUV 36493. INLET TEMP॰ (DEG. K) 293.0 , PRESSURE (ATM。) 1.000

CALIBRATION CORRECTION: COUNT/TRUE COUNT

INLET: $1.0000+0.0$ *YNTI + 0.0 *YTRI/YIVTI

OUTLET: $1.1800+-0.1800$ YINTO + 0.0 \#YTRNO/YINTJ

GAS

HE
(INERT)

$\begin{array}{llll}\text { MOLECD WEIGHT } & \text { (G/GMOLE) } & 4.000 & 17.03 \\ \text { DENSITY } & \text { (KG/M*3) } & 0.1648 & 0.7182 \\ \text { VISCOSITY } & (K G / M / S E C) & 1.9410 E-05 & 9.8200 E-06 \\ \text { VEAV FREE PATH } & (M) & 1.8860 E-07 & 4.5700 E-08 \\ \text { VOLUME } \$(1 / 3) & (M / G M O L E *(1 / 3)) & 2.2880 E-02 & 2.2240 E-02\end{array}$

DIFFUSI VITY (M\%2/SEC)

$5.8859 E-05$

\begin{tabular}{|c|c|c|c|c|c|c|c|}
\hline $\begin{array}{l}\text { RUN } \\
\text { TYPE }\end{array}$ & $\begin{array}{l}\text { YINTI } \\
\text { YINTO } \\
\text { YINTLM }\end{array}$ & $\begin{array}{l}\text { PEXP } \\
\text { PCORR } \\
M T\end{array}$ & $\begin{array}{l}\text { EPMO } \\
\text { EPSW } \\
\text { EPMA. }\end{array}$ & $\begin{array}{l}\text { QINT } \\
\text { PLM } \\
\text { ULM }\end{array}$ & $\begin{array}{l}\text { FILYR } \\
\text { KN } \\
\text { UR }\end{array}$ & $\begin{array}{l}\text { REI } \\
\text { REO } \\
\text { PELM }\end{array}$ & $c$ \\
\hline 6486 & $\begin{array}{l}0.3030 \\
0.7630 \\
0.5369 \\
0.4440\end{array}$ & $\begin{array}{l}.6689 \\
.6824\end{array}$ & $\begin{array}{l}6029 \\
7144 \\
7848\end{array}$ & $\begin{array}{l}O E-04 \\
O E-08 \\
4 \\
F-04\end{array}$ & $\begin{array}{l}1 \\
5 \\
0\end{array}$ & $\begin{array}{l}2026 . \\
3200 \\
7590 \\
2005\end{array}$ & $1.3589 E-02$ \\
\hline 6 & $\begin{array}{l}0 . \\
0 . \\
0 .\end{array}$ & & $\begin{array}{l}64 \\
88 \\
60\end{array}$ & $\begin{array}{l}4.7900 E-04 \\
7.7890 E-08 \\
1.488\end{array}$ & $\begin{array}{l}0.1 \\
0.0\end{array}$ & $\begin{array}{r}2005 . \\
502 . \\
959 .\end{array}$ & 02 \\
\hline 6491 & $\begin{array}{l}0.5 \\
0.8 \\
0.8\end{array}$ & $\begin{array}{l}0.4140 \\
0.4317 \\
0.7641\end{array}$ & $\begin{array}{l}591 \\
941 \\
41\end{array}$ & $\begin{array}{l}6.7600 E-04 \\
8.5554 E-08 \\
1.929\end{array}$ & $\begin{array}{l}0.2 \\
0.2 \\
0.0\end{array}$ & $\begin{array}{r}2019 . \\
6390 \\
1093 .\end{array}$ & 2.02 \\
\hline 6491 & $\begin{array}{l}0.5 \\
0.8 \\
0.6\end{array}$ & & $\begin{array}{l}0.3591 \\
0.4941 \\
0.6041\end{array}$ & $\begin{array}{l}6.7600 E-04 \\
8.5554 E-08 \\
1.929\end{array}$ & $\begin{array}{l}0.2 \\
0.2 \\
0.0\end{array}$ & $\begin{array}{r}2019^{\circ} \\
639 . \\
1093 .\end{array}$ & $2.0218 E-02$ \\
\hline 64 & $\begin{array}{l}0.6340 \\
0.8340 \\
0.7424\end{array}$ & & & $\begin{array}{l}1.0390 E-03 \\
9.4024 E-08 \\
2.762\end{array}$ & $\begin{array}{l}0.2 \\
0.2 \\
0.0\end{array}$ & $\begin{array}{r}2090^{\circ} \\
954^{\circ} \\
1380\end{array}$ & -02 \\
\hline res & $\begin{array}{l}0.6340 \\
0.8340 \\
0.7424\end{array}$ & $\begin{array}{l}0.3521 \\
0.3709 \\
0.6552\end{array}$ & $\begin{array}{l}0.2398 \\
0.3562 \\
0.4657\end{array}$ & $\begin{array}{l}1.0390 E-03 \\
9.4024 E-08 \\
2.762\end{array}$ & $\begin{array}{l}0.2 \\
0.2 \\
0.0\end{array}$ & $\begin{array}{r}2090^{\circ} \\
954^{\circ} \\
1380^{\circ}\end{array}$ & 2 \\
\hline 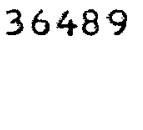 & $\begin{array}{l}0.6 \\
0.8 \\
0.7\end{array}$ & $\begin{array}{l}0.2147 \\
0.2345 \\
0.6130\end{array}$ & $\begin{array}{l}0.1857 \\
0.2899 \\
0.3980\end{array}$ & $\begin{array}{l}1.3800 E-03 \\
1.0230 E-07 \\
3.476\end{array}$ & $\begin{array}{l}0.1 \\
0.2 \\
0.0\end{array}$ & $\begin{array}{l}2185^{\circ} \\
1155 \circ \\
1558 .\end{array}$ & -02 \\
\hline 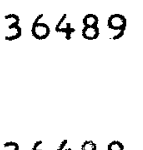 & $\begin{array}{l}0.6 \\
0.8 \\
0.7\end{array}$ & $\begin{array}{l}0.2260 \\
0.2456 \\
0.6130\end{array}$ & $\begin{array}{l}0.1857 \\
0.2899 \\
0.3980\end{array}$ & $\begin{array}{l}1.3800 E-03 \\
1.0230 E-07 \\
3.476\end{array}$ & $\begin{array}{l}0.1 \\
0.2 \\
0.0\end{array}$ & $\begin{array}{l}2185^{\circ} \\
1155^{\circ} \\
1558 .\end{array}$ & .4533 \\
\hline 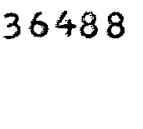 & $\begin{array}{l}0.7440 \\
0.8680 \\
0.8112\end{array}$ & $\begin{array}{l}0.1975 \\
0.2161 \\
0.5580\end{array}$ & $\begin{array}{l}0.1429 \\
0.2317 \\
0.3316\end{array}$ & $\begin{array}{l}1.7440 E-03 \\
1.0875 E-07 \\
4.243\end{array}$ & $\begin{array}{l}0.1779 \\
0.2753 \\
0.0034\end{array}$ & $\begin{array}{l}2302 . \\
1386^{\circ} \\
1760^{\circ}\end{array}$ & $6044 E$ \\
\hline
\end{tabular}


HE , $\mathrm{NH} 3$

- 0.790 MICRON D

PARTICLES (CONTINUED).

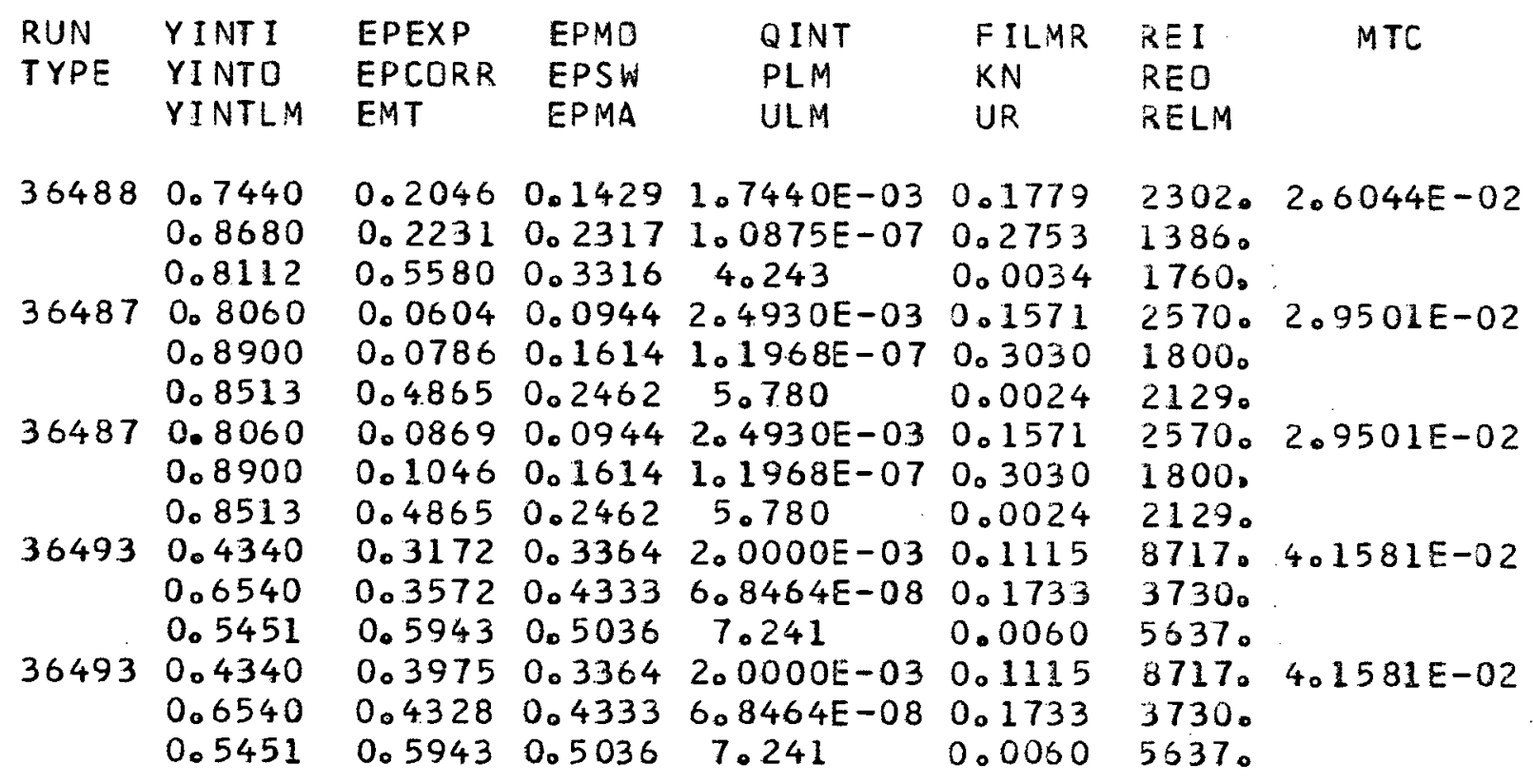


TABLE XII

DATA FOR CH4 , NH3 O 0.790 MICRON DIAYETER PARTICLES。

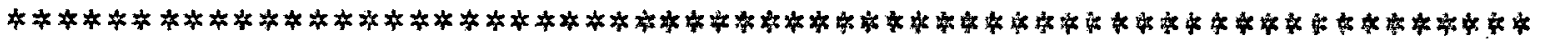

INERT GAS FLJW RATE CONSTANT IN ALL RUNS。

INLET TEMP。 (DEG。.K) 293.0 , PRESSURE (ATM。) 1.000

CAL IBRAT ION CORRECTION: COUNT/TRUE COUNT

INLET: $1.0000+0.0$ *YNTI +0.0 \#YTRNI/YIVTI

JUTLET: $1.0000+0.0$ \#YINTO + 0.0 YYRNO/YINTO

GAS

$\begin{array}{cc}\text { CH4 } & \text { VH3 } \\ \text { (INERT) } & \text { (TRANSFERRED) }\end{array}$

$\begin{array}{llll}\text { MOLEC。 WEIGHT } & \text { (G/GMOLE) } & 16.03 & 17.03 \\ \text { DENSITY } & \text { (KG/M*3) } & 0.6678 & 0.7182 \\ \text { VISCOSITY } & \text { (KG/M/SEC) } & 1.0870 E-05 & 9.8200 E-06 \\ \text { MEAN FREE PATH } & (\text { M) } & 5.2500 E-08 & 4.5700 E-08 \\ \text { VOLUME } \$(1 / 3) & \text { (M/GMOLE } \$ 1 / 3)) & 3.2900 E-02 & 2.2240 E-02\end{array}$

DIFFUSIVITY (M*2/SEC)

$2.4684 E-05$

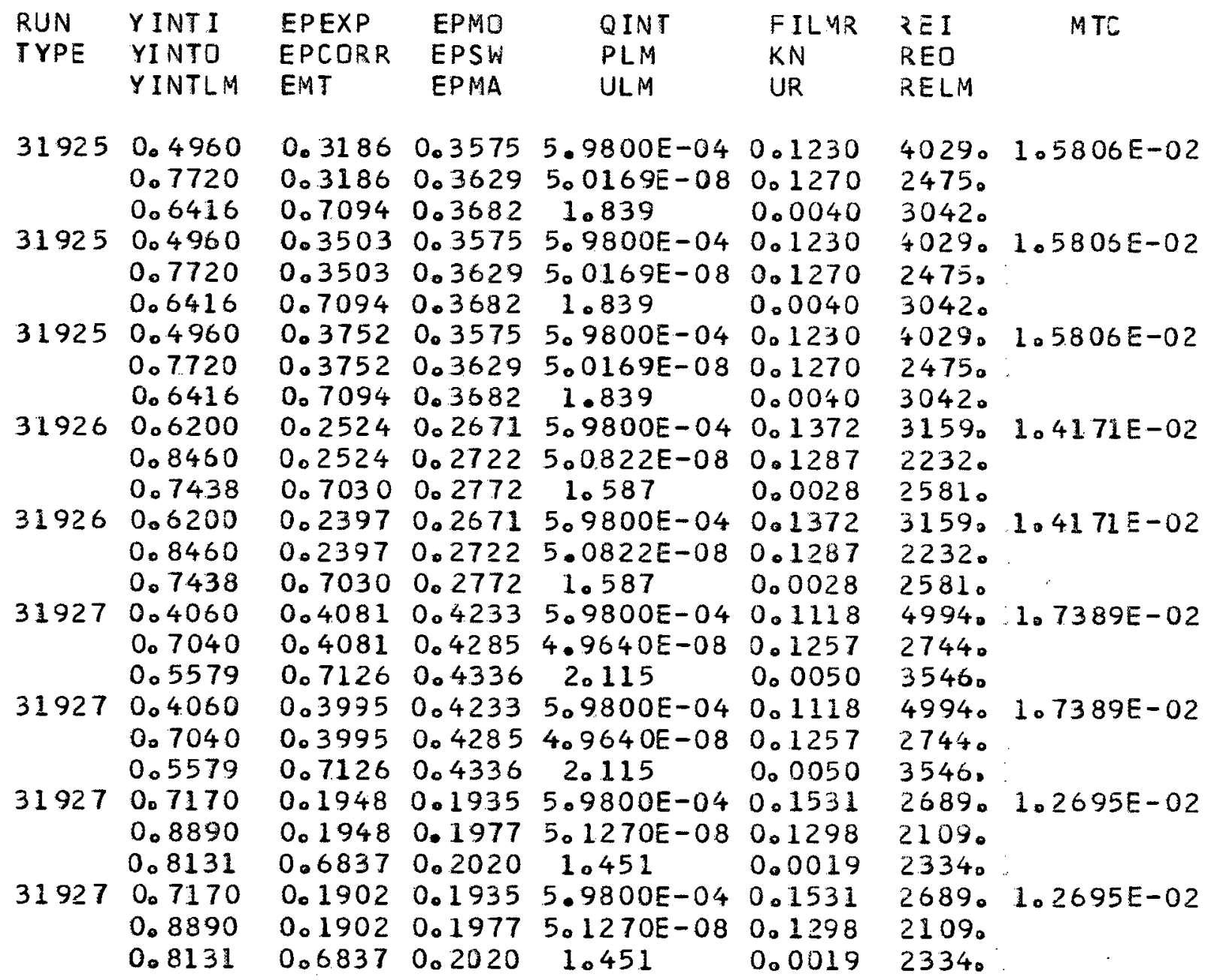




\begin{tabular}{|c|c|c|c|c|c|c|c|}
\hline $\mathrm{CH}_{4}$ & , $\mathrm{NH} 3$ & - & $790 \mathrm{M}$ & RON D. PAF & T I CLES & (CONTI & UEDI。 \\
\hline $\begin{array}{l}\text { RUN } \\
\text { TYPE }\end{array}$ & $\begin{array}{l}\text { YINTI } \\
\text { YINTO } \\
\text { YINTLM }\end{array}$ & $\begin{array}{l}\text { EPEXP } \\
\text { EPCORR } \\
\text { EMT }\end{array}$ & $\begin{array}{l}\text { EPMO } \\
\text { EPSW } \\
\text { EPMA }\end{array}$ & $\begin{array}{l}\text { QINT } \\
\text { PLM } \\
\text { ULM }\end{array}$ & $\begin{array}{l}\text { FILMR } \\
\text { KN } \\
\text { UR }\end{array}$ & $\begin{array}{l}\text { REI } \\
\text { REO } \\
\text { RELM }\end{array}$ & MTC \\
\hline 31928 & $\begin{array}{l}0.3460 \\
0.6400\end{array}$ & $\begin{array}{l}0.4837 \\
0.4837\end{array}$ & $\begin{array}{l}0.4594 \\
0.4642\end{array}$ & $\begin{array}{l}5.9800 E-04 \\
4.9226 E-08\end{array}$ & $\begin{array}{l}0.1068 \\
0.1246\end{array}$ & 5917 . & $1.8201 E-02$ \\
\hline 31928 & $\begin{array}{l}0.3411 \\
0.3460 \\
0.6400 \\
0.4917\end{array}$ & $\begin{array}{l}0.4744 \\
0.4744 \\
0.7024\end{array}$ & $\begin{array}{l}0.4009 \\
0.4594 \\
0.4642 \\
0.4689\end{array}$ & $\begin{array}{l}5.9800 E-04 \\
4.9226 E-08 \\
2.400\end{array}$ & $\begin{array}{l}0.1005 \\
0.1068 \\
0.1246 \\
0.0055\end{array}$ & $\begin{array}{l}400 \% \\
3917 \% \\
3050 \% \\
+067 \%\end{array}$ & $1.3201 E-02$ \\
\hline 31928 & $\begin{array}{l}0.2520 \\
0.5360 \\
0.3861\end{array}$ & $\begin{array}{l}0.5447 \\
0.5447 \\
0.7084\end{array}$ & $\begin{array}{l}0.5299 \\
0.5339 \\
0.5378\end{array}$ & $\begin{array}{l}5.9800 E-04 \\
4.8574 E-08 \\
3.056\end{array}$ & $\begin{array}{l}0.0904 \\
0.1230 \\
0.0059\end{array}$ & $\begin{array}{l}8249 . \\
3704{ }^{\circ} \\
5268^{\circ}\end{array}$ & $2.1504 E-02$ \\
\hline 31928 & $\begin{array}{l}0.2520 \\
0.5360 \\
0.3861\end{array}$ & $\begin{array}{l}0.5293 \\
0.5293 \\
0.7084\end{array}$ & $\begin{array}{l}0.5299 \\
0.5339 \\
0.5378\end{array}$ & $\begin{array}{l}5.9800 E-04 \\
4.8574 E-08 \\
3.056\end{array}$ & $\begin{array}{l}0.0904 \\
0.1230 \\
0.0059\end{array}$ & $\begin{array}{l}8249^{\circ} \\
3704 \circ \\
5268 .\end{array}$ & $.2 .1504 E-02$ \\
\hline
\end{tabular}


TABLE XIII

DATA FOR N2 - $\mathrm{NH} 3$

- 0.790 MICRON DIAMETER PARTICLES-

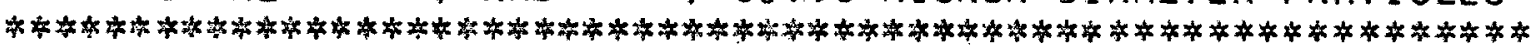
PRELIMINARY RUNS。

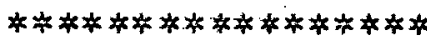

DATA FOR VARIOUS TYPES OF RUNS。

INLET TEMP。 (DEG。K) 293.0 , PRESSURE (ATM。) 1.000

CALIBRATION CORRECTION: COUNT/TRUE COUNT

INLET: $1.0000+0.0$ \#INTI + -0.0420\%YTRNI/YINTI

OUTLET: $1.0000+0.0$ *YINTO + -0.0420*YTRNO/YINTO

GAS

N2
(INERT

$\begin{array}{llll}\text { MOLEC \& WEIGHT } & \text { (G/GMOLE) } & 28.02 & 17.03 \\ \text { DENSITY } & (K G / M * 3) & 1.165 & 0.7182 \\ \text { VISCOSITY } & (K G / M / S E C) & 1.7480 E-05 & 9.8200 E-06 \\ \text { MEAN FREE PATH } & (M) & 6.3900 E-08 & 405700 E-08 \\ \text { VOLUME }(1 / 3) & (M / G M O L E \$(1 / 3)) & 3.1190 E-02 & 2.2240 E-02\end{array}$

DIFFUS IVITY $(M * 2 / S E C)$

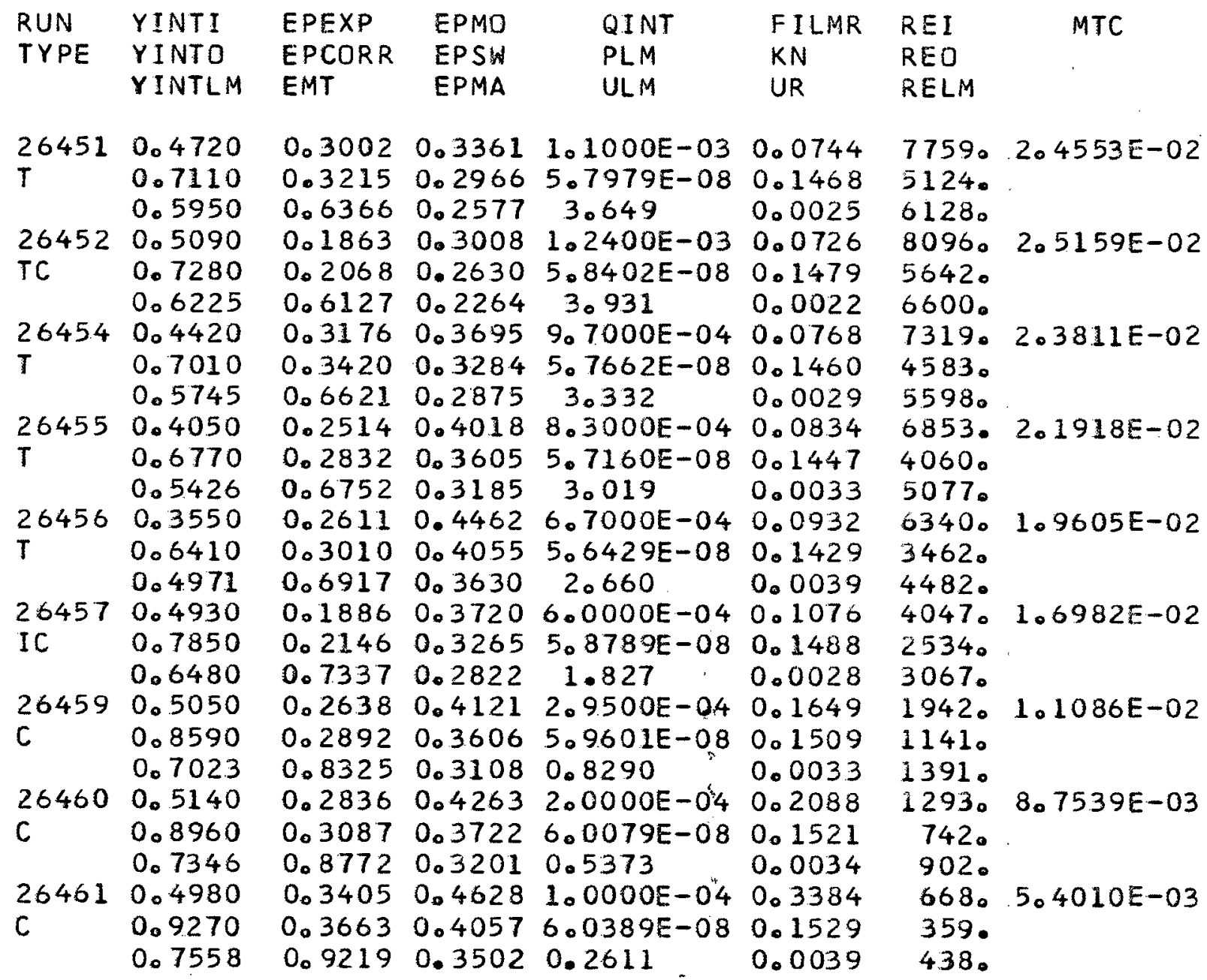


N2 - $\mathrm{NH} 3$
-

0.790 MICRON D。

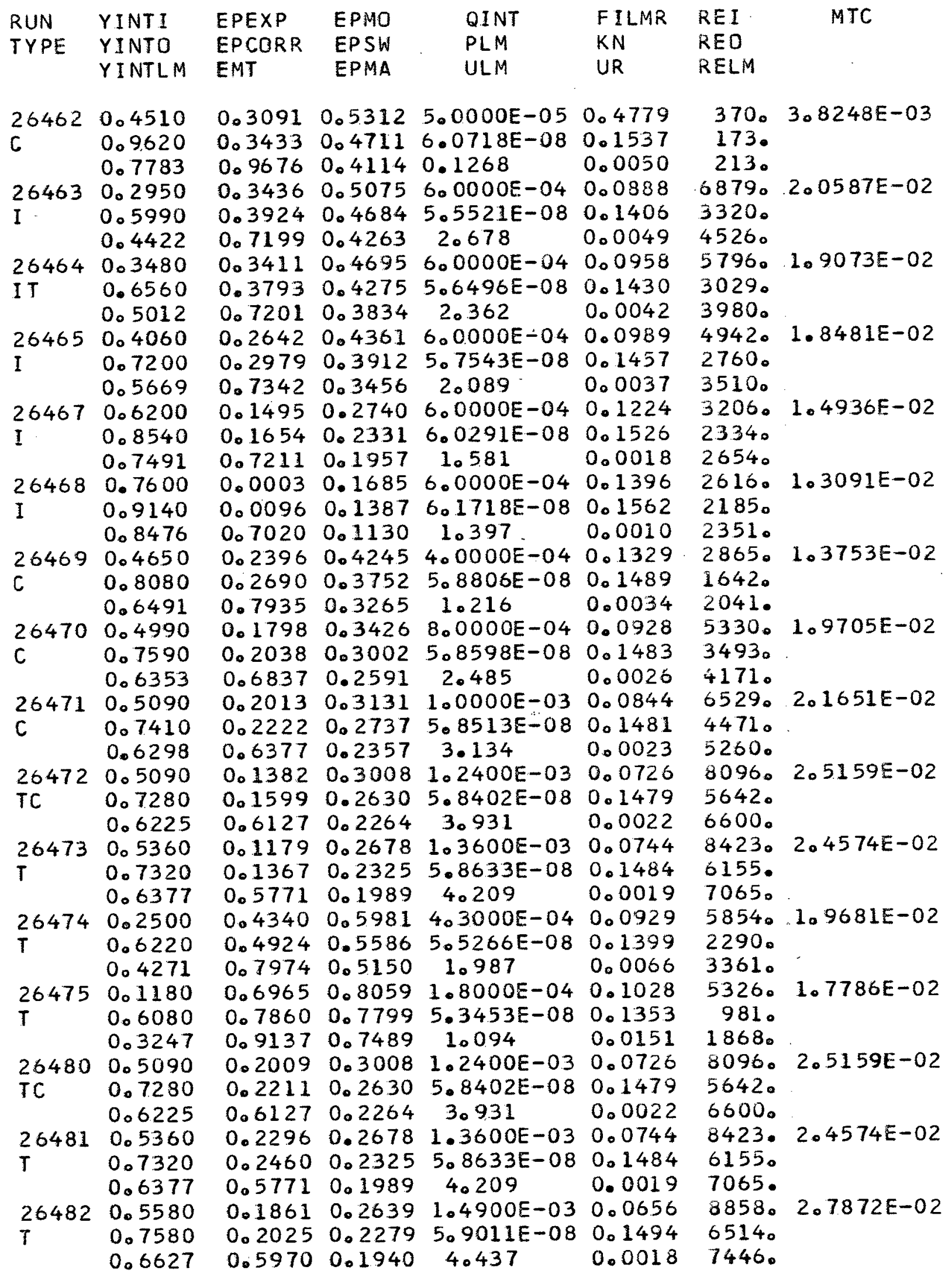


N2 - $\mathrm{NH3}$ - 0.790 MICRON

D. PARTICLES (CONTINUED)。

\begin{tabular}{|c|c|c|c|c|c|c|c|}
\hline $\begin{array}{l}\text { UN } \\
\text { YPE }\end{array}$ & $\begin{array}{l}\text { YINTI } \\
\text { YINTO } \\
\text { YINTLM }\end{array}$ & $\begin{array}{l}\text { EPEXP } \\
\text { EPCORR } \\
\text { EMT }\end{array}$ & $\begin{array}{l}\text { EPMO } \\
\text { EPSW } \\
\text { EPMA }\end{array}$ & $\begin{array}{l}\text { QINT } \\
\text { PLM } \\
\text { ULM }\end{array}$ & $\begin{array}{l}\text { F ILMR } \\
\text { KN } \\
\text { UR }\end{array}$ & $\begin{array}{l}\text { REI } \\
\text { RED } \\
\text { RELM }\end{array}$ & MTC \\
\hline $\begin{array}{l}26483 \\
26484\end{array}$ & $\begin{array}{l}0.4720 \\
0.7110 \\
0.5950 \\
0.3550 \\
0.6410 \\
0.4971\end{array}$ & $\begin{array}{l}0.2692 \\
0.2914 \\
0.6366 \\
0.3398 \\
03755 \\
0.6917\end{array}$ & $\begin{array}{l}0.3361 \\
0.2966 \\
0.2577 \\
0.4462 \\
0.4055 \\
0.3630\end{array}$ & $\begin{array}{l}1.1000 E-03 \\
5.7979 E-08 \\
3.649 \\
6.7000 E=04 \\
5.6429 E-08 \\
2.660\end{array}$ & $\begin{array}{l}0.0744 \\
0.1468 \\
0.0025 \\
0.0932 \\
0.1429 \\
0.0039\end{array}$ & $\begin{array}{l}7759 . \\
51240^{\circ} \\
6128^{\circ} \\
6340^{\circ} \\
34620^{\circ} \\
4482^{\circ}\end{array}$ & $.9605 E-02$ \\
\hline $\begin{array}{l}26485 \\
26486\end{array}$ & $\begin{array}{l}0.4420 \\
0.7010 \\
0.5745 \\
0.4050 \\
0.6770 \\
0.5426\end{array}$ & $\begin{array}{l}0.2526 \\
0.2793 \\
0.6621 \\
0.2988 \\
0.3286 \\
0.6752\end{array}$ & $\begin{array}{l}0.3695 \\
0.3284 \\
0.2875 \\
0.4018 \\
0.3605 \\
0.3185\end{array}$ & $\begin{array}{l}9.7000 E-04 \\
5.7662 E-08 \\
3.332 \\
8.3000 E-04 \\
5.7160 E-08 \\
3.019\end{array}$ & $\begin{array}{l}0.0768 \\
0.1460 \\
0.0029 \\
0.0834 \\
0.1447 \\
0.0033\end{array}$ & $\begin{array}{l}7319^{\circ} \\
4583 . \\
5598 \circ \\
6853 \circ \\
4060 . \\
5077^{\circ}\end{array}$ & $2.1918 E-02$ \\
\hline
\end{tabular}


TABLE XIV

DATA FOR N2 - $\mathrm{NH} 3$

- 0.790 MICRON DIAMETER PARTICLES-

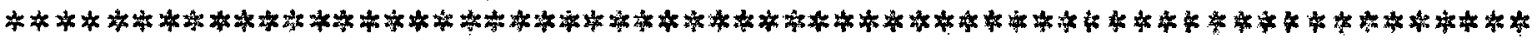

SHORT COLUMN。

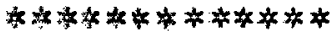

INERT GAS FLOW RATE CONSTANT IN ALL RUNS。

INLET TEMP。 (DEG。 K) 293.0 , PRESSURE (ATM。) 1.000

CALIBRATION CORRECTION: COUNT/TRUE COUNT

INLET: $\quad 1.0000+0.0$ *YINTI + 0.0 *YTRNI/YINTI

OUTLET: $1.0900+-0.0900 *$ YINTO + 0.0 *YTRNO/YINTO

GAS

$\begin{array}{ll}\text { N2 } & \text { NH3 } \\ \text { (INERT) } & \text { (TRANSFERRED) }\end{array}$

$\begin{array}{llcc}\text { MOLEC。 WEIGHT } & \text { (G/GMOLE) } & 28.02 & 17.03 \\ \text { DENSITY } & \text { (KG/M*3) } & 1.165 & 0.7182 \\ \text { VISCOSITY } & (K G / M / S E C) & 1.7480 E-05 & 9.8200 E-06 \\ \text { MEAN FREE PATH } & (M) & 6.3900 E-08 & 4.5700 E-08 \\ \text { VOLUME } \$(1 / 3) & (M / G M O L E *(1 / 3)) & 3.1190 E-02 & 2.2240 E-02\end{array}$

DIFFUSIVITY $\quad(M * 2 / S E C)$

$2.3212 E-05$

$\begin{array}{llllllll}\text { RUN } & \text { YINTI } & \text { EPEXP } & \text { EPMO } & \text { QINT } & \text { FILMR } & \text { REI } & \text { MTC } \\ \text { TYPE } & \text { YINTO } & \text { EPCORR } & \text { EPSW } & \text { PLM } & \text { KN } & \text { REO } & \\ & \text { YINTLM } & \text { EMT } & \text { EPMA } & \text { ULM } & \text { UR } & \text { RELM }\end{array}$

$37068 \quad 0.2610$

$0.2507 \quad 0.3203$

0.3840

0.2901

0.2983

$6.0000 E-04 \quad 0.0722$

$7811 \cdot .2 .5311 E-02$

0.3200

0.4334

0.2741

5. $3367 E-08$

0.1351

5234 。

370680.2610

0.24610 .3203

3.700

0.0065

0.3840

0.2857

0.2983

6.0000E-04 0.0722

0.3200

0.4334

0.2741

$5.3367 E=08$

0.1351

6321 。

$7811.2 .5311 E-02$

370680.2610

$0.2507 \quad 0.3203$

3.700

0.0065

0.3840

0.2901

0.2983

6. $0000 E-04$

0.0722

0.3200

0.4334

0.2741

5. $3367 E-08$

0.1351

370690.3480

0.2710

0.3095

3.700

0.0065

0.5040

0.3022

0.2818

6.0000E-04

0.0750

0.4244

0.4747

0.2528

$5.5220 E-08$

0.1398

2.790

0.0059

370690.3480

0.2256

0.3095

6. OOOOE- 04

0.0750

0.5040

0.2587

0.2818

5.5220E-08

0.1398

52340

6321 。

0.4244

0.4747

0. 2528

2. 790

0.0059

7811 。

5234 。

63210

5796. 2.4385E-02

3957 。

4721 。

5796. 2.4385E-02

3957 .

4721 。

370690.3480

0.2298

0.3095

6.0000E-04 0.0750

5796 。

0. 5040

0. 2627

0.2818

$5.5220 E-08$

0.1398

3957 .

0.4244

0.4747

0.2528

2.790

0.0059

4721 。

$37070 \quad 0.4100$

0.1861

0.2807

6.0000E-04

0.0811

0.5700

0.2164

0.2515

$5.6307 E-08$

0.1425

0.4896

0.4758

0.2220

2. 419

0.0051

$37070 \quad 0.4100$

0.1961

0.2807

$6.0000 E-04$

0.0811

0.5700

0.2261

0. 2515 5.6307E-08

0.1425

0.4896

0.4758

0.2220

2.419

0.0051

370710.4980

0.1564

$0.24096 .0000 E-04$

0.0881

0.6560

0.1817

$0.2111507719 E-08$

0.1451

4892 。

3491 。

4076 。

4892 .

3491 。

4076 。

4006. 2.0743E-02

0.5782

0.4798

$0.1823 \quad 2.048$

0.0040

3029 .

3441 。 
N2 $\quad$ NH3 $\quad 0.790$ MICRON D. PARTICLES (CONT INUED)。

\begin{tabular}{|c|c|c|c|c|c|c|c|}
\hline $\begin{array}{l}\text { RUN } \\
\text { TYPE }\end{array}$ & $\begin{array}{l}\text { YINTI } \\
\text { YINTO } \\
\text { YINTLM }\end{array}$ & $\begin{array}{l}\text { EPEXP } \\
\text { EPCORR } \\
\text { EMT }\end{array}$ & $\begin{array}{l}\text { EPMO } \\
\text { EPSW } \\
\text { EPMA }\end{array}$ & $\begin{array}{l}\text { QINT } \\
\text { PLM } \\
\text { ULM }\end{array}$ & $\begin{array}{l}\text { FILMR } \\
\text { KN } \\
\text { UR }\end{array}$ & $\begin{array}{l}\text { REI } \\
\text { RED } \\
\text { RELM }\end{array}$ & MTC \\
\hline 37071 & $\begin{array}{l}0.4980 \\
0.6560\end{array}$ & $\begin{array}{l}0.1880 \\
0.2124\end{array}$ & $\begin{array}{l}0.2409 \\
0.2111\end{array}$ & $\begin{array}{l}6.0000 E-04 \\
5.7719 E-08\end{array}$ & $\begin{array}{l}0.0881 \\
0.1461\end{array}$ & $\begin{array}{l}4006 . \\
3029 .\end{array}$ & $2.0743 E-02$ \\
\hline 37071 & $\begin{array}{l}0.5782 \\
0.4980 \\
0.6560 \\
0.5782 \\
0.6210\end{array}$ & $\begin{array}{l}0.4798 \\
0.1903 \\
0.2146 \\
0.4798\end{array}$ & $\begin{array}{l}0.1823 \\
0.2409 \\
0.2111 \\
0.1823\end{array}$ & $\begin{array}{l}2.048 \\
6.0000 E-04 \\
5.7719 E-08 \\
2.048\end{array}$ & $\begin{array}{l}0.0040 \\
0.0881 \\
0.1461 \\
0.0040\end{array}$ & $\begin{array}{l}34410 \\
4006 \% \\
3029 \circ \\
3441 \%\end{array}$ & $2.0743 E-02$ \\
\hline 37072 & $\begin{array}{l}0.6210 \\
0.7560 \\
0.6912 \\
0.6210\end{array}$ & $\begin{array}{l}0.1464 \\
0.1647 \\
0.4712 \\
0.1322\end{array}$ & $\begin{array}{l}0.1786 \\
0.1519 \\
0.1275 \\
0.1786\end{array}$ & $\begin{array}{l}6.0000 E-04 \\
5.9436 E-08 \\
1.713 \\
6.0000 E-04\end{array}$ & $\begin{array}{l}0.0999 \\
0.1505 \\
0.0027 \\
0.0999\end{array}$ & $\begin{array}{l}3201 \% \\
2630 \% \\
2875 \circ \\
3201\end{array}$ & $1.8292 E-02$ \\
\hline 37072 & $\begin{array}{l}0.6210 \\
0.7560 \\
0.6912 \\
0.6210 \\
0.7560 \\
0.6912\end{array}$ & $\begin{array}{l}0.1322 \\
0.1508 \\
0.4712 \\
0.1145 \\
0.1335 \\
0.4712\end{array}$ & $\begin{array}{l}0.1786 \\
0.1519 \\
0.1275 \\
0.1786 \\
0.1519 \\
0.1275\end{array}$ & $\begin{array}{l}6.0000 E-04 \\
5.9436 E-08 \\
1.713 \\
6.0000 E-04 \\
5.9436 E-08 \\
1.713\end{array}$ & $\begin{array}{l}0.0999 \\
0.1505 \\
0.0027 \\
0.0999 \\
0.1505 \\
0.0027\end{array}$ & $\begin{array}{l}32011^{\circ} \\
2630 \circ \\
2875 \circ \\
3201 \% \\
2630 \circ \\
2875 \circ\end{array}$ & $1.8292 E-02$ \\
\hline
\end{tabular}


TABLE XV

DATA FOR N2

, $\mathrm{NH3}$

- 0.500 MICRON DIAMETER PARTICLES。

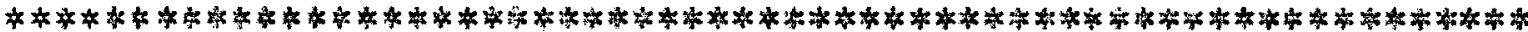

INERT GAS FLOW RATE CONSTANT IN ALL RUNS。

INLET TEMP。 (DEG。K) 29300 , PRESSURE (ATM。) 1.000

CALIBRATION CORRECTION: COUNT/TRUE COUNT

INLET: $\quad 1.0000+0.0$ *YINTI + 0.0 *YTRNI/YINTI

OUTLET: $0.8769+0.1231$ *INTO + 0.0 *YTRNO/YIVTJ

GAS

\section{N2 \\ (INERT)}

$\begin{array}{ll}\text { MOLEC。WEIGHT } & (\text { G/GAOLE) } \\ \text { DENSITY } & (K G / M * \$ 3) \\ \text { VISCOSITY } & (K G / M / S E C) \\ \text { MEAN FREE PATH } & (M) \\ \text { VOLUME } *(1 / 3) & (M / G M O L E *(1 / 3))\end{array}$

DIFFUS IVITY

$(M * 2 / S E C)$

$\begin{array}{ll}28.02 & 17.03 \\ 1.165 & 0.7182 \\ 1.7480 E-05 & 9.8200 E-06 \\ 6.3900 E-08 & 4.5700 E-08 \\ 3.1190 E-02 & 2.2240 E-02\end{array}$

$2.3212 E-05$

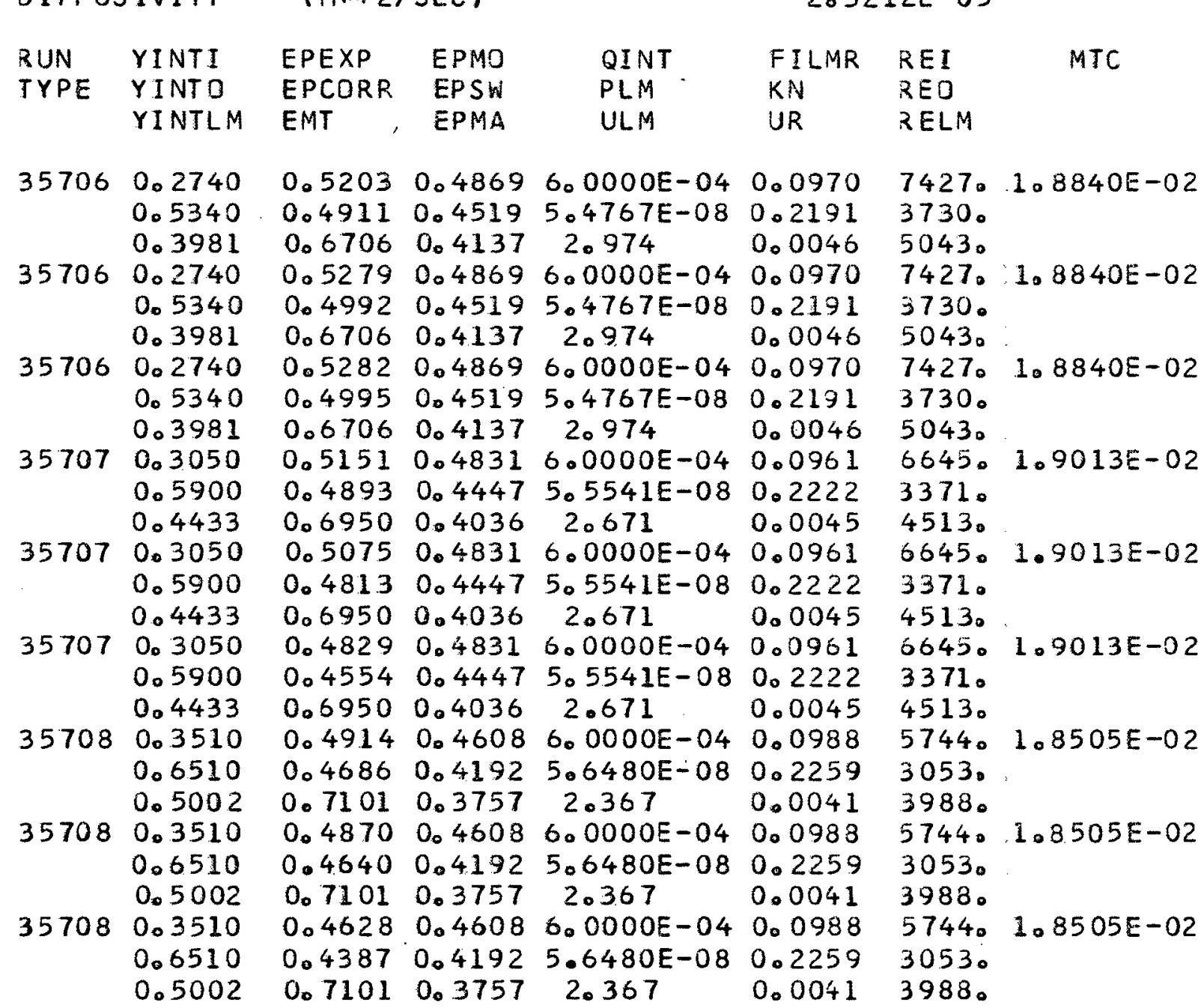

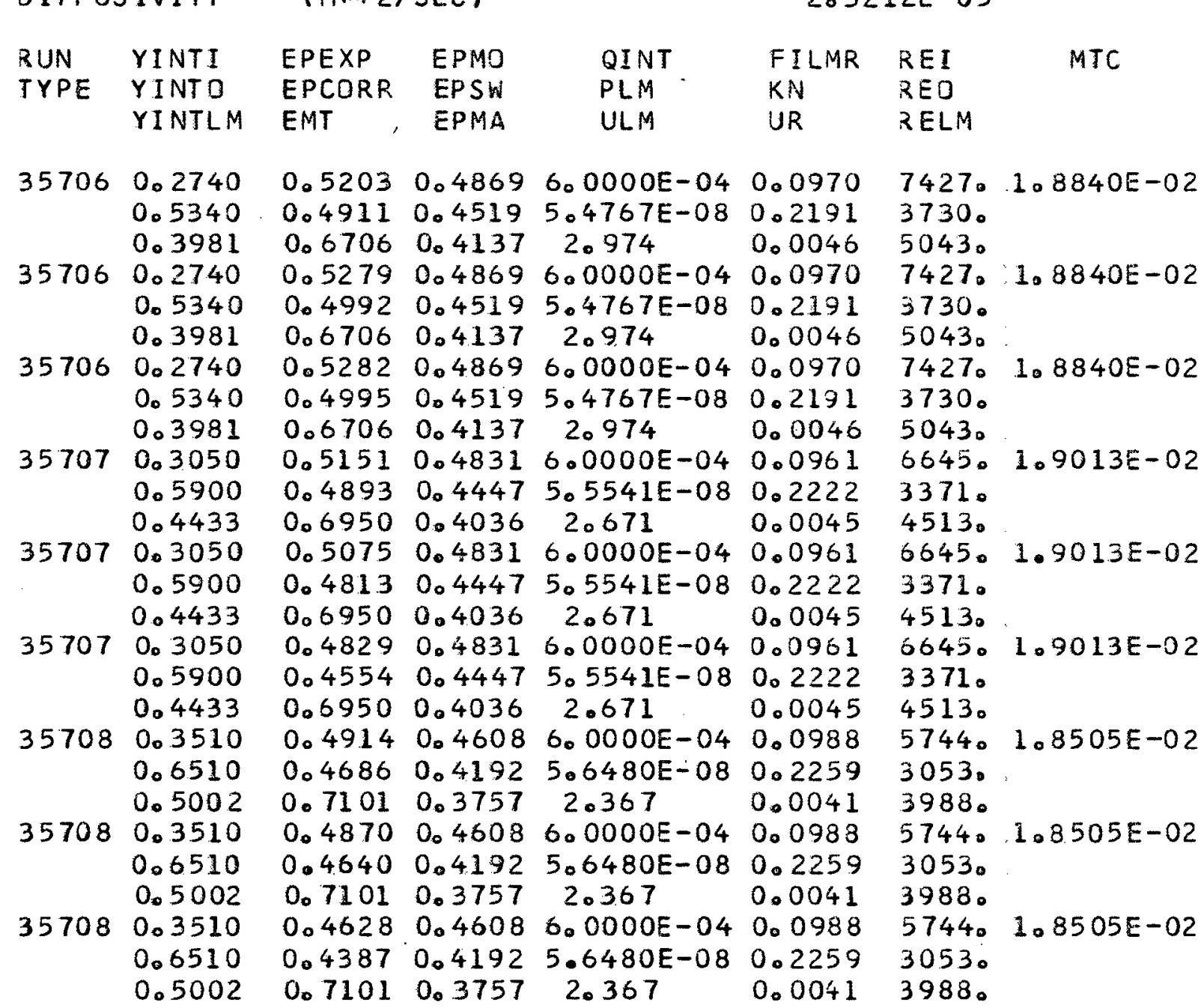

$\mathrm{NH3}$

(T3ANSFERRED)

$$
\begin{aligned}
& 17.03 \\
& 0.7182 \\
& 9.8200 E-06 \\
& 4.5700 E-08 \\
& 2.2240 E-02
\end{aligned}
$$




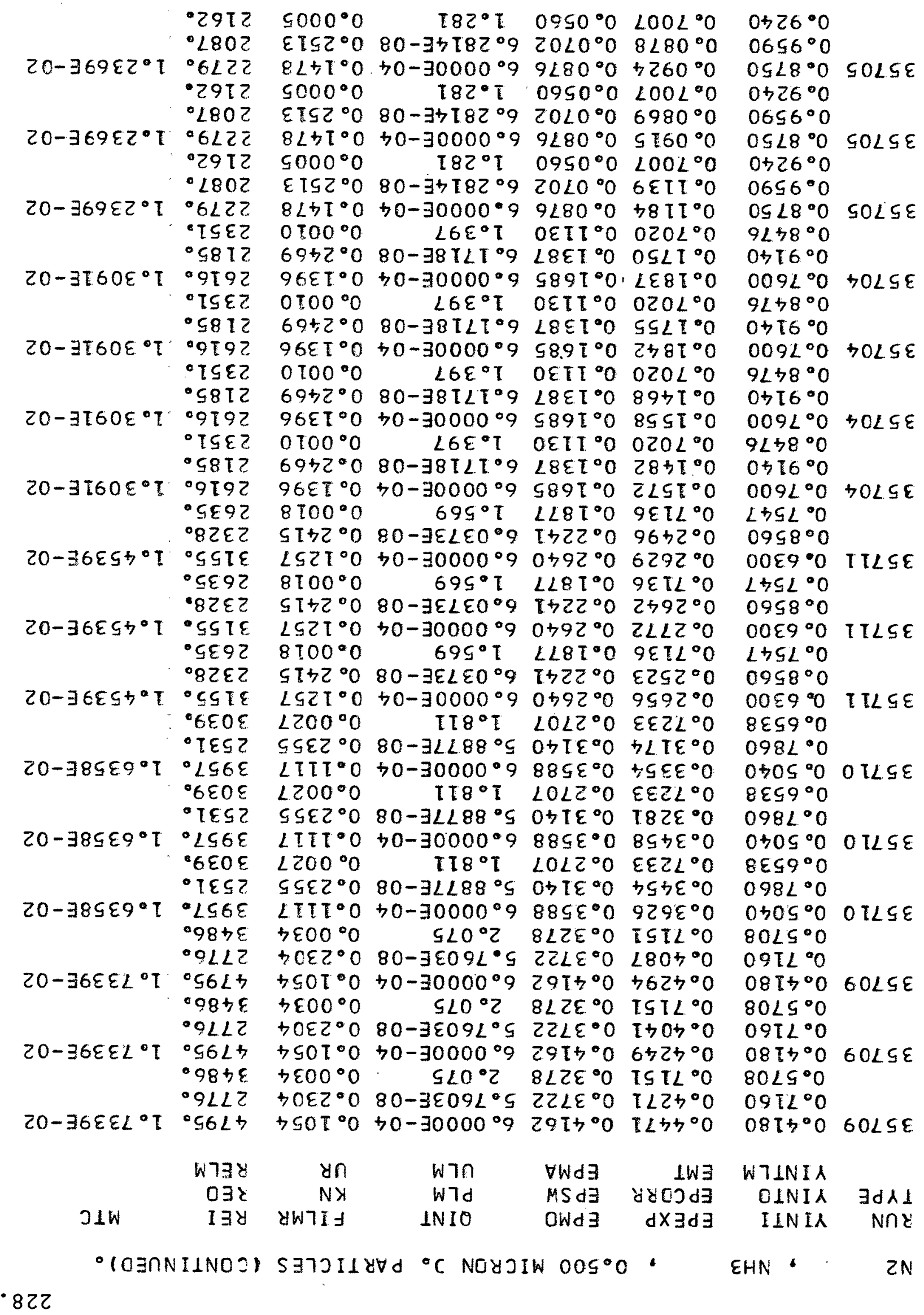


TABLE XVI

DATA FOR N2

, $\mathrm{NH3}$

- 0.790 MICRON DIAMETER PARTICLES。

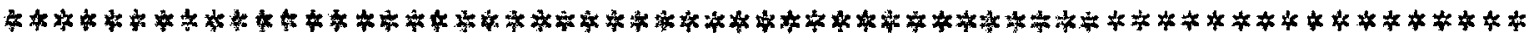

DATA FOR VARIOUS TYPES OF RUNS。

INLET TEMP。 IDEG。K) 293.0 , PRESSURE (ATM。) 1.000

CALIBRATION CORRECTION: COUNT/TRUE COUNT

INLET: $\quad 100000+0.0$ *YINTI + 0.0 *YTRNI/YINTI

OUTLET: $0.8992 * 0.1008$ *YNTO + 0.0 *YTRNO/YINTJ

GAS

N2
(INERT

$\begin{array}{llcc}\text { MOLEC。WEIGHT } & \text { (G/GMOLE) } & 28.02 & 17.03 \\ \text { DENSITY } & \text { (KG/M*3) } & 1.165 & 0.7182 \\ \text { VISCOSITY } & (\text { KG/M/SEC) } & 1.7480 E-05 & 9.8200 E-06 \\ \text { MEAN FREE PATH } & (M) & 6.3900 E-08 & 4.5700 E-08 \\ \text { VOLUME }(1 / 3) & (M / G M D L E *(1 / 3)) & 3.1190 E-02 & 2.2240 E-02\end{array}$

DIFFUSIVITY (M* $2 /$ SEC

2. $3212 E-05$

$\begin{array}{llllllll}\text { RUN } & \text { YINTI } & \text { EPEXP } & \text { EPMD } & \text { QINT } & \text { FILMR } & \text { REI } & \text { MTC } \\ \text { TYPE } & \text { YINTO } & \text { EPCORR } & \text { EPSW } & \text { PLM } & \text { KN } & 3 E O & \\ & \text { YINTLM } & \text { EMT } & \text { EPMA } & \text { ULM } & \text { UR } & \text { RELM }\end{array}$

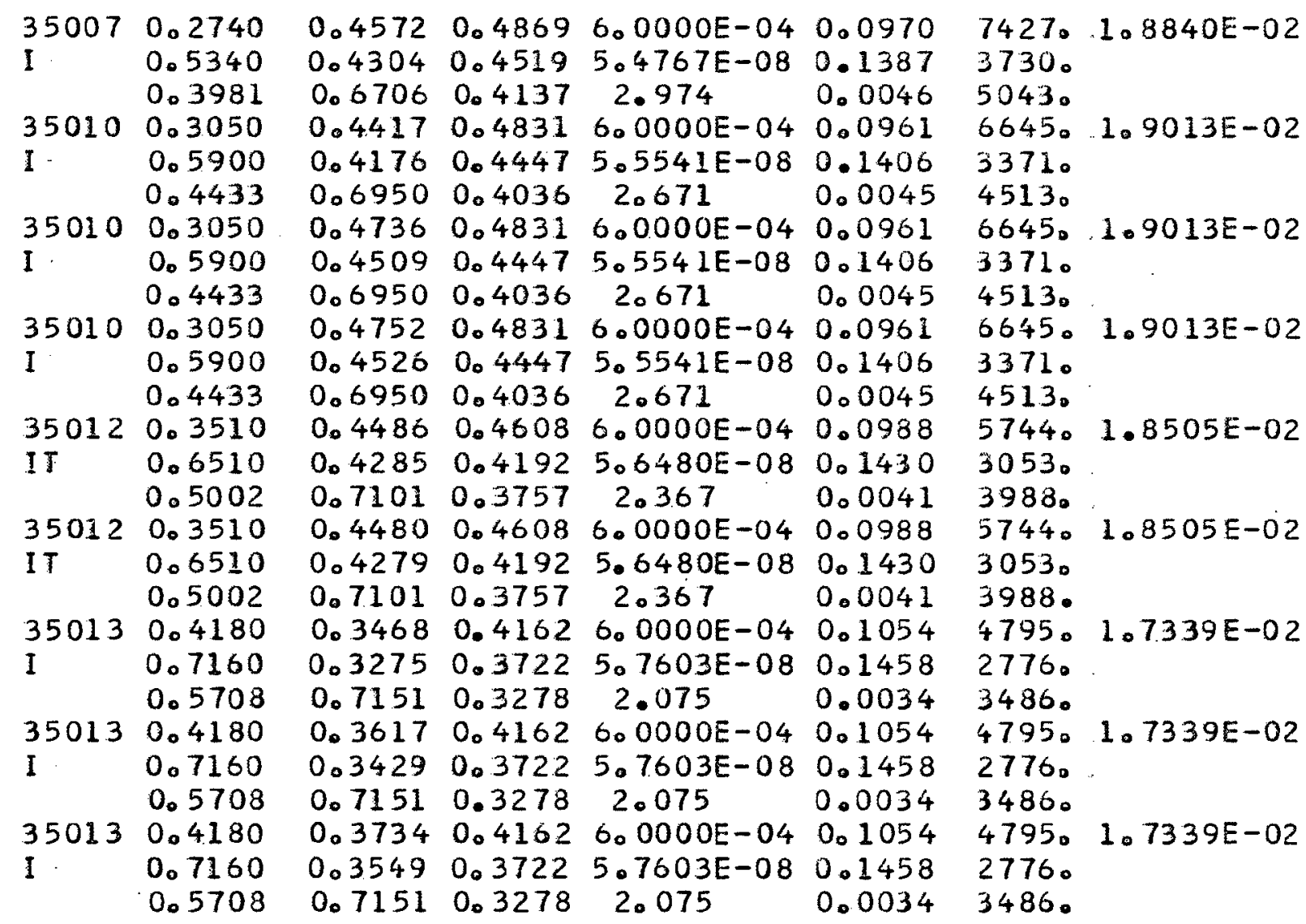


N2 - NH3

0.790 MICRON D.

PARTICLES

( SONTINUED).

$\begin{array}{ll}\text { RUN } & \text { YINTI } \\ \text { TYPE } & \text { YINTO } \\ & \text { YINTLM } \\ & \\ 35011 & 0.5540 \\ \text { IC } & 0.7860 \\ 35011 & 0.6538 \\ \text { IC } & 0.5040 \\ & 0.6560 \\ & 0.658\end{array}$

350110.5040

IC 0.7860

0.6538

350090.6300

I 0.8560

0.7547

350090.6300

I $\quad 0.8560$

0.7547

350090.6300

I 0.8560

0.7547

350230.7600

I 0.9140

0.8476

350230.7600

I 0.9140

0.8476

350230.7600

I 0.9140

0.8476

350230.7600

I. 0.9140

0.8476

350230.7600

I. 0.9140

0.8476

350230.7600

I. 0.9140

0.8476

350240.8750

$1 \quad 0.9590$

0.9240

350240.8750

I 0.9590

0.9240

350240.8750

I 0.9590

0.9240

350250.2740

1

0.5340

0.3981

\section{EPEXP EPMO}

EPCORR EPSW

EMT

0.3162

0.3011

0.7233

0.3074

0.2921

0.7233

0.2980

0.2825

0.7233

0.2074

0.1957

0.7136

0.2225

0.2110

0.7136

0.2342

0.2229

0.7136

0.1381

0.1306

0.7020

0.1225

0.1148

0.7020

0.1256

0.1180

0.7020

0.1247

0.1170

0.7020

0.1311

0.1235

0.7020

0.1301

0.1225

0.7020

0.0096

0.0055

0.7007

0.0668

0.0629

0.7007

0.0465

0.0425

0.7007

0.4839

0.4585

0.6706

EPMA

0.1130

0. 1585

0.1387

0.1387

0.1130

0. 1130

0.1685

0.1387

0.1130

0.1685

0.1387

0.1130

0.0876

0.0702

0.0560

0.0876

0.0702

0.0560

0.0876

0.0702

0.0560

0.4869

0.4519

0.4137
QINT
PLM
ULM

FILMR

$K N$

UR

0.3588 6.0000E-04 0.1117

$0.3140 \quad 5.8877 E-08$

$0.2707 \quad 1.811$

0.1491

0.1117

$0.31405 .8877 E-08 \quad 0.1491$

$0.2707 \quad 1.811$

0.0027

0.3588 6. DOOOE-04 0.1117

$0.3140 \quad 5.8877 E-08 \quad 0.1491$

$0.2707 \quad 1.811$

0.0027

0.2640 6.0000E-04 0.1257

$0.22416 .0373 E-080.1528$

$0.1877 \quad 1.569$

0.0018

$0.26406 .0000 E-040.1257$

$0.22416 .0373 E-08 \quad 0.1528$

$0.1877 \quad 1.569$

0.0018

$0.26406 .0000 E-040.1257$

$0.22416 .0373 E-08 \quad 0.1528$

$0.1877 \quad 1.569$

0.0018

0.1685 6. OOOOE-04 0.1396

0.1387 6.1718E-08 0.1562

0.1130

1.397

0.0010

6.0000E-04 0.1396

$6.1718 E-08 \quad 0.1562$

1.397

0.0010

0.1685

6.000 OE-04

0.1396

6.1718E-08 0.1562

1.397

0.0010

6.000OE-04 0.1396

$6.1718 E-08 \quad 0.1562$

1.397

0.0010

6.000OE-04

$6.1718 E-08$

1.397

0.1396

6.000OE-04

6. $1718 \mathrm{E}-08$

1.397

0.1562

0.0010

$6.0000 E-04$

0.1396

0.1562

0.0010

6. $2814 E-08$

1.281

0.1478

6.0000E -04

0.1590

0.0005

$000 E-040.1478$

. $2814 E-08$

1.281

6. OOOOEE-04 0.1478

6. $2814 \mathrm{E}-08 \quad 0.1590$

1.281

6. 0000E-04

$5.4767 E-08 \quad 0.1387$
2.974
REI

REO

RELM

3957, 1.6358E-02

2531 。

3039.

3957. 1.6358E-02

2531 .

3039 。

3957.1.6358E-02

2531 。

30390

3155。1.4539E-02

2328 .

2635 。

3155.1.4539E-02

2328 .

2635 。

3155.1.4539E-02

2328 。

2635 。

2616.1.3091E-02

2185.

2351 。

2616 。

2185 。

2351 。

2616。1.3091E-02

2185 。

2351 .

2616。.1.3091E-02

2185 。

2351.

2616.1.3091E-02

2185.

$2351 。$

2616。1.3091E-02

2185 .

2351 。

2279.1.2369E-02

2087 。

2162 .

$1.2369 E-02$
0.1590

0.0005

0.0005

0.0970

2279 。

2087 .

2162 .

2279 。

2087 .

2162 .

7427.1.8840E-02

$3730^{\circ}$

5043 。

$1.2369 E-02$

0.0046

$1.8840 E-02$ 
N2 - NH3

$\checkmark$

0.

$\begin{array}{ll}\text { RUN } & \text { YINTI } \\ \text { TYPE } & \text { YINTO } \\ & \text { YINTLM } \\ 35025 & 0.2740 \\ \text { I } & 0.5340 \\ & 0.3981\end{array}$

350250.2740

I 0.5340

0. 3981

350250.2740

I $\quad 0.5340$

0.3981

350250.2740

I. 0.5340

0.3981

350250.2740

I . 0.5340

0.3981

$35026 \quad 0.3050$

I 0.5900

0.4433

350260.3050

I 0.5900

0.4433

350260.3050

I 0.5900

0.4433

350270.3510

IT 0.6510

0.5002

350270.3510

IT 0.6510

0.5002

350270.3510

IT 0.6510

0.5002

350370.1180

T 0.6080

0.3247

350370.1180

T 0.6080

0.3247

350310.3550

T 0.6410

0.4971

350370.1180

$T \quad 0.6080$

0.3247

350370.1180

T 0.6080

0.3247
EPEXP EPMO

EPCORR EPSW

EMT

0.4957

0.4708

0.6706

0.4693

0.4431

0.6706

0.4886

0.4634

0.6706

0.4736

0.4477

0.6706

0.4408

0.4132

0.6706

0.4428

0.4188

0.6950

0.4318

0.4073

0.6950

0.4152

0.3900

0.6950

0.3823

0.3598

0.7101

0.4261

0.4052

0.7101

0.4307

0.4099

0.7101

0.7825

0.7736

0.9137

D. 7839

0.7750

0.9137

0.2762

0.2490

0.6917

0.7921

0.7835

0.9137

0.7815

0.7725

0.9137

EPMA
$0.48696 .0000 E-04 \quad 0.0970$

$0.4519 \quad 5.4767 E-08 \quad 0.1387$

0.41372 .974

0.0046

$0.48696 .0000 E-040.0970$

$0.4519 \quad 5.4767 E-08 \quad 0.1387$

0.41372 .974

0.0046

0.4869 6. OOOOE-04 0.0970

$0.45195 .4767 E-08 \quad 0.1387$

$0.4137 \quad 2.974$

0.0046

0.4869 6.00OOE-04 0.0970

$0.45195 .4767 E-08 \quad 0.1387$

$0.4137 \quad 2.974$

0.0046

$0.48696 .0000 E-040.0970$

$0.45195 .4767 E-08 \quad 0.1387$

0.41372 .974 .000046

$0.48316 .0000 E-040.0961$

$0.44475 .5541 E-08 \quad 0.1406$

$\begin{array}{lll}0.4036 & 2.671 & 0.0045\end{array}$

0.4831 6.0000E-04 0.0961

$0.44475 .5541 E-080.1406$

$0.4036 \quad 2.671 \quad 0.0045$

0.4831 6.0000E-04 0.0961

0.4447 5.5541E-08 0.1406

$0.4036 \quad 2.671$

0.0045

0.4608 6. O0OOE-04 0.0988

0.4192 506480E-08 0.1430

$0.37572 .367 \quad 0.0041$

$\begin{array}{lll}0.4608 & 6.0000 E-04 & 0.0988\end{array}$

$0.41925 .6480 E-08 \quad 0.1430$

$\begin{array}{lll}0.3757 & 2.367 & 0.0041\end{array}$

0.4608 6.0000E-04 0.0988

$0.41925 .6480 E-080.1430$

$\begin{array}{lll}0.3757 & 2.367 & 0.0041\end{array}$

0.8059 1.8000E-04 0.1028

$0.77995 .3453 E-08 \quad 0.1353$

$\begin{array}{lll}0.7489 & 1.094 & 0.0151\end{array}$

$0.80591 .8000 E-040.1028$

$0.77995 .3453 E-08 \quad 0.1353$

$\begin{array}{lll}0.7489 & 1.094 & 0.0151\end{array}$

$0.44626 .7000 E-040.0932$

$0.40555 .6429 E-080.1429$

$\begin{array}{lll}0.3630 & 2.660 & 0.0039\end{array}$

$0.80591 .8000 E-040.1028$

0.7799 5.3453E-08 0.1353

$0.7489 \quad 1.094$

0.0151

$0.80591 .8000 E-040.1028$

$0.77995 .3453 E-08 \quad 0.1353$

$0.7489 \quad 1.094$

0.0151
(CONT INUED).

REI
REO
RELM

7427, 1.8840E-02

3730 。

5043.

7427。1.8840E-02

3730 。

5043 。

7427. 1.8840E-02

3730 。

5043 。

7427. 1.8840E-02

3730 。

5043 。

7427. 1.8840E-02

3730 。

5043 .

6645.1.9013E-02

3371 .

4513 。

6645.1.9013E-02

3371 。

45130

6645. 1.9013E-02

3371 。

4513.

$5744.1 .8505 E-02$

3053.

3988 。

5744。 1.8505E-02

3053.

3988 。

5744。1.8505E-02

3053 。

3988 。

5326. 1.7786E-02

981 .

1868 .

5326. 1.7786E-02

981.

1868 。

6340 .

3462 。

4482 。

$5326.1 .7786 E-02$

981 。

1868 .

5326. 1.7786E-02

981 。

1868. 
N2 , NH3

RUN

YINTI

YINTO

YINTLM

EPEXP

,

0.790 MICRON D.

PART ICLES

TYPE

$\begin{array}{ll}35033 & 0.4420 \\ T & 0.7010\end{array}$

0.5745

350330.4420

T 0.7010

0.5745

350330.4420

T 0.7010

0.5745

350340.4720

$T \quad 0.7110$

0.5950

350340.4720

T 0.7110

0.5950

350340.4720

$\mathrm{T} \quad 0.7110$

0.5950

350350.5090

TC 0.7280

0.6225

350350.5090

TC 0.7280

0.6225

350350.5090

TC 0.7280

0.6225

350350.5090

TC 0.7280

0.6225

350360.2500

$\mathrm{T} \quad 0.6220$

0.4271

350360.2500

T 0.6220

0.4271

$35036 \quad 0.2500$

$T \quad 0.6220$

0.4271

350380.3550

$T \quad 0.6410$

0.4971

350380.3550

T 0.6410

0.4971

350380.3550

T 0.6410

0.4971
EPCORR EPSW

EMT EPMA

0.2931

0.3695

0.2711

0.6621

0.2915

0.2695

0.6621

0.3175

0.2963

0.6621

0.3106

0.2899

0.6366

0.3033

0.2824

0.6366

0.3090

0.2883

0.6366

0.2875

0.2674

0.6127

0.2727

0.2522

0.6127

0.3284

0.2875

0.3695

0.3284

0.2875

0.3695

0.3284

0.2875

0. 3361

0.2966

0.2577

0.3361

0.2966

0.2577

0.3361

0.2966

0.2577

0.3008

0.2630

0.2264

0.3008

0.2630

0.2264

0.3008

0.2675

0.2468

0.6127

0.2630

0.2264

0.2655

0.2448

0.6127

0.3008

0.2630

0.2264

0.5441

0.5260

0.7974

0.5981

0.5586

0.5150

0.5594

0.5419

0.7974

0.5981

0.5586

0.5150

0.5718

0.5548

0.7974

0.4564

0.4360

0.6917

0.4463

0.4255

0.6917

0.4310

0.4096

0.6917

\section{QINT \\ PLM \\ ULM}

FILMR
KN
UR

$9.7000 E-04 \quad 0.0768$

5.7662E-08 0.1460

3.332

0.0029

$9.7000 E-04 \quad 0.0768$

5.7662E-08 0.1460

3.332

0.0029

9.7000E-04 0.0768

5.7662E-08 0.1460

3.332

0.0029

1. $1000 E-03 \quad 0.0744$

5.7979E-08 0.1468

3.649

0.0025

1. $1000 E-03 \quad 0.0744$

5.7979E-08 0.1468

3.649

0.0025

1. $1000 E-03$ 0.0744

$5.7979 E-08 \quad 0.1468$

3. 649

0.0025

1.2400E-03 0.0726

0.1479

3. 931

0.0022

1.2400E-03 0.0726

$5.8402 E-08$

3. 931

0.1479

0.0022

$1.2400 E-03 \quad 0.0726$

5. $8402 E-08$

3.931

0.1479

0.0022

$1.2400 E-030.0726$

$5.8402 E-08$

3.931

0.1479

0.0022

4.3000E-04 0.0929

5.5266E-08 0.1399

1.987

0.0056

4. $3000 E-04 \quad 0.0929$

5. 5266E-08 0.1399

1.987

0.0066
5.8402E-08
(CONT I NUED).

REI
REO
RELM

$7319 \cdot 2.3811 E-02$

4583 。

5598.

7319.2.3811E-02

4583 。

5598.

$7319.2 .3811 E-02$

4583 。

5598.

$7759.2 .4553 E-02$

5124 。

6128 。

7759.2.4553E-02

5124 。

5128 。

7759.2.4553E-02

5124 。

6128 。

8096. 2.5159E-02

5642 .

6600 。

8096.2.5159E-02

5642 .

6600 .

8096. 2.5159E-02

5642 .

6600 。

8096. 2.5159E-02

5642 。

6600 。

$585401.9681 \mathrm{E}-02$

2290 。

3361 。

$585401.9681 \mathrm{E}-02$

2290 。

3361 。
0.5981

0.5586

0.5150

0.4462

0.4055

0.3630

0.4462

0.4055

0.3630

4.3000E-04 0.0929

$5.5266 E-080.1399$

1.9 .87

0.0066

6.7000E-04 0.0932

$5.6429 E-080.1429$

2.660

0.0039

6.7000E-04 0.0932

$5.6429 E-08 \quad 0.1429$

2.660

0.0039

0.4462

0.4055

6.7000E-04 0.0932

$5.6429 E-08 \quad 0.1429$

2.660

0.0039

5854 。

2290 。

3361.

6340 。

3462 .

4482 。

6340 。

3462 .

4482 。

6340。.1.9605E-02

3462 。

4482 。

1. $9681 E-02$

$1.9605 E-02$

1. $9605 E-02$

0.3630 
$N 2$ - $\mathrm{NH3}$

RUN YINTI

TYPE YINTO YINTLM

EPEXP EPCORR EMT

350380.3550

T $\quad 0.6410$ 0.4971

350380.3550

T $\quad 0.6410$ 0.4971

350390.4050

T 0.6770 0.5426

350390.4050

I $\quad 0.6770$ 0.5426

350390.4050

T 0.6770 0.5426

350390.4050

T 0.6770 0.5426

$35040 \quad 0.4720$

T 0.7110 0.5950

$35040 \quad 0.4720$

$\mathrm{T} \quad 0.7110$

0.5950

$35040 \quad 0.4720$

T $\quad 0.7110$

0.5950

$35040 \quad 0.4 .720$

T 0.7110

0.5950

350410.5090

TC 0.7280 0.6225

350410.5090

TC 0.7280 0.6225

350410.5090

TC 0.7280

0.6225

350420.1180

T 0.6080

0.3247

350420.1180

$T \quad 0.6080$

0. 3247

350420.1180

T 0.6080 0.3247

(1)

0.4014

0.6917

0.4077

0.3855

0.6917

0.3820

0.3612

0.6752

0.3713

0.3501

0.6752

0.3597

0.3381

0.6752

0.3802

0.3593

0.6752

0.3088

0.2881

0.6366

0.2789

0.6366

0.3042

0.2833

0.6366

0.2878

0.2664

0.6366

0.2850

0.2659

0.6127

0.2859

0.2658

0.6127

0.2902

0.2702

0.6127

0.8146

0.8070

0.9137

0.7917

0.7831

0.9137

0.7790

0.7699

0.9137
0.790 MICRON D. PARTICLES

\section{EPMO}

EPSW

EPMA

QINT
PLM
ULM

FILMR
KN
UR

$0.42310 .4462 \quad 607000 E-040.0932$

$0.40555 .6429 E-08$

0.1429

$0.3630 \quad 2.660$

0.0039

0.4462

$6.7000 E-04$

0.0932

0.4055

$5.6429 E-08$

0.1429

$0.3630 \quad 2.660$

0.0039

0.4018

8.3000E-04

0.0834

0.3605

. $7160 E-08$

0.1447

0.31853 .019

0.0033

0.3605

8.3000E-04

0.0834

0.3185

5. $7160 E-08$

0.1447

3.019

0.0033

0.4018

8. $3000 E-04$

0.0834

0.3605

5.7160E-08

0.1447

0.31853 .019

0.0033

0.4018

8. $3000 E-04$

0.0834

0.3605

5.7160E-08

0.1447

0.3185

3.019

0.0033

0.3361

1. $1000 E-03$

0.0744

0.2966

5.7979E-08

0.1468

0.2999

0.2577

3.649

0.0025

0.2966

1. $1000 E-03$

0.0744

0.2577

$5.7979 E-08$

0.1468

0.0025

0.2966

$1.1000 E-03$

0.0744

0.2577

5.7979E-08

0.1468

0.0025

0.3361

3.649

0.0744

0.2966

1000E-O3

0.1468

0.2577

5.7979E-08

0.0025

0.3008

3.649

0.0726

0.2630

$1.2400 E-03$

0.1479

0.2264

5.8402E-08

3.931

0.0022

0.3008

$1.2400 E-03$

0.0726

0.2630

5. 8402E-0 8

0.1479

0.22643 .931

0.0022

0.3008

$1.2400 E-03$

0.0726

0.2630

5. $8402 E-08$

0.1479

0.2264

3.931

0.0022

0.8059

1. $8000 E-04$

0.1028

0.7489

$5.3453 E-08$

0.1353

0.8059

1.094

0.0151

0.7799

1. $8000 E-04$

0.1028

0.7489

5.3453E-08

0.1353

1.0094

0.0151

0.8059

1. $8000 E-04$

0.1028

0.7799

$5.3453 E-08$

0.1353

0.0151

(CONTIVUED)。

REI
REO
RELM

MTC

6340。 1.9605E-02

3462 。

4482 .

$6340.1 .9605 E-02$

3462 。

4482 .

$6853.2 .1918 E-02$

4060 。

5077 .

6853.2.1918E-02

4060。

5077 .

$6853.2 .1918 E-02$

4060 .

5077 。

6853 .

4060 。

5077 。

7759.

5124 。

5128 .

$7759^{\circ}$

5124 .

5128.

7759 。

51240

6128 。

7759.2.4553E-02

5124.

0128.

8096. 2.5159E-02

5642 .

6600 .

8096. 2.5159E-02

5642 。

6600 。

8096 .

5642 。

6600 。

5326 。

981 。

1868 . 
N2 - NH3

RUN

YINTI

YINTO

YINTLM

$\begin{array}{ll}\text { EPEXP } & \text { EPMO } \\ \text { EPCORR } & \text { EPSW } \\ \text { EMT } & \text { EPMA }\end{array}$

QINT

PLM

ULM

PARTICLES

(:ONTINUED)。

350420.1180

0.8042

0.8059

0.7961

0.7799

1. $8000 E-04$

0.1028
0.1353
0.0151

0.3247

0.9137

0.7489

$.3453 E-08$

1.094

FILMR
KN

UR

0.5417

0.5981

0.5235

40

0.5586

4. 3000E-04

0.0929

T 0.6220

0.4271

350430.2500

0.7974

0.5981

.5266 E-08

0.1399

0.5487

0.5308

T. 0.4271

0.7974

0.5586

1.987

0.0066

350430.2500

T 0.6220

0.4271

0.5445

(1)

0.7974

0.5150

4.3000E-04

0.0929

.5266 E-08

0.1399

0.0066

350440.5090

C 0.7410

0.6298

0.2928

0.5981

1.987

0.0929

0.5586

4.3000E-04

0.1399

0.5150

1.987

0.0066

0.2738

0.6377

$0.31311 .0000 E-03$

0.0844

$0.27375 .8513 E-080.1481$

0.2806

350440.5090

C 0.7410

0.6298

0.2613

0.2357

3.134

0.0023

0.6377

0.3131 1.0000E-03

0.0844

0.2784

350440.5090

C 0.7410

0.6298

350440.5090

0.2591

0.6377

0.2737

5. $8513 E-08$

0.1481

$0.2357 \quad 3.134$

0.0023

0.3131

1. OOOOE-03

0.0844

$0.27375 .8513 E-080.1481$

0.3195

$0.2357 \quad 3.134$

0.0023

C 0.7410

0.6298

0.3013

0.6377

0.3131

1.000

0.0844

0.2737

0.2357

$5.8513 E-08$

0.1481

0.2755

0.2575

0.3426

3.134

0.0023

350450.4990

$6 \quad 0.7590$

0.6353

$35045 \quad 0.4990$

C 0.7590

0.6353

350450.4990

C 0.7590

0.6353

0.6837

0.3002

8.0OOOE-04

0.0928

0.2647

0.2591

$5.8598 E-08$

2. 485

0.1483

0.2464

0.6837

0.3426

8.0000E-04

0.0026

0.3002

0.2591

$5.8598 E-08$

2.485

0.0928

0.2758

0.3426

8.0000E-04

0.1483

0.2578

0.6837

0.3002

0.2591

$5.8598 E-08$

2.485

0.0026

0.0928

0.2651

0.3426

8.0000E-04

0.1483

0.0026

350460.4990

0.7590

0.2468

0.6837

0.3002

5. 8598E-08

2. 485

0.0928

0.2591

350460.4990

C $\quad 0.7590$

0.6353

$35046 \quad 0.4990$

0.2781

8.0000E-04

0.1483

0.0026

0.2601

0.6837

0.2842

0.2664

0.6837

0.6353

350470.4650

C $\quad 0.8080$

0.6491

350470.4650

C 0.8080

0.6491
0.3555

0.3428

0.7935

0.3503

0.3375

0.7935
0.3426

0.3002 5.8598E-08

0.25912 .485

0.0928

0.3426

0.3002

8.0000E-04

0.1483

0.0026

40.0928

0.2591

0.4245

0.3752

0.3265

0.4245

0.3752

0.3265
. 8598E-08

2.485

0.1483

.0000E-04 0.1329

5.8806E-08 0.1489

$1.216 \quad 0.0034$

4. O000E-04 0.1329

$5.8806 E-08 \quad 0.1489$

1.216

0.0034
REI
REO
RELM

5326. 1.7786E-02

981 。

1868 。

5854。 1.9681E-02

2290 。

3361 。

5854。.1.9681E-02

2290 。

3361 。

5854 . 1.9681E-02

2290 。

3361 。

$6529.2 .1651 E-02$

4471 。

5260。

5529.2.1651E-02

4471 。

5260 。

6529.2.1651E-02

4471 。

5260 .

6529.2.1651E-02

4471 。

5260 。

5330。1.9705E-02

3493 。

4171 。

$5330, .1 .9705 E-02$

3493 。

4171 .

5330. 1.9705E-02

3493 。

4171 。

5330。1.9705E-02

3493 。

4171 。

5330。1.9705E-02

3493 。

4171 。

5330.1.9705E-02

3493 。

4171 。

$2865 \circ 1.3753$ E-02

1642 。

2041 .

2365.1.3753E-02

1642 。

2041 . 


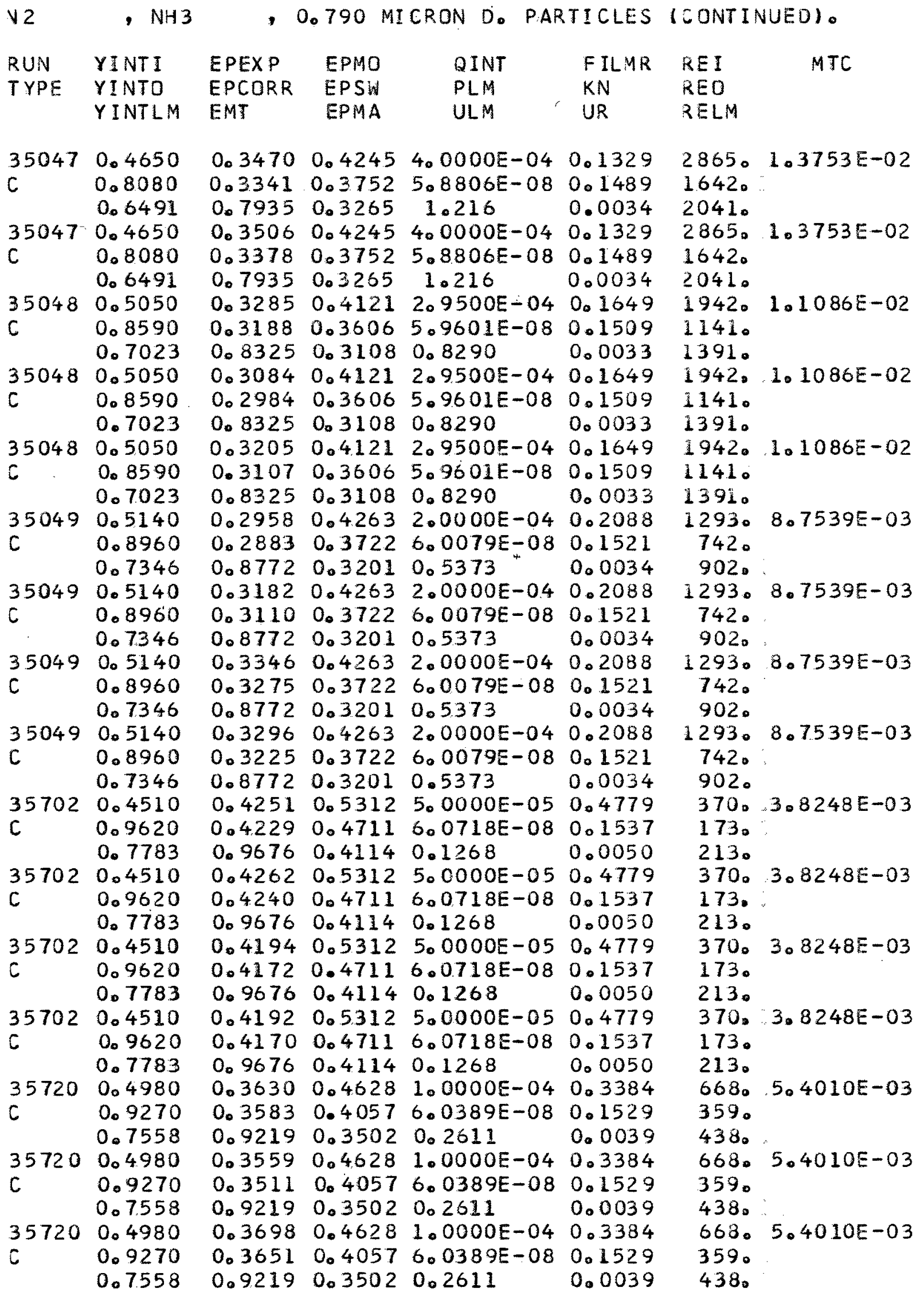


$\mathrm{N} 2$ , $\mathrm{NH} 3$

RUN Y INT I

TYPE YINTO YINTLM

EPEXP

EPCORR

EMT

$35720 \quad 0.4980$

C 0.9270

0.7 .558

357210.5090

C 0.7410

0.6298

357210.5090

C 0.7410

0.6298

357210.5090

c

0.7410

0.6298

357210.5090

C

0.7 .410

0.6298

357220.5090

TC

0.7280

0.6225

357220.5090

TC

0. 7280

0.6225

357220.5090

TC 0.7280

0.6225

357220.5090

TC 0.7280

0.6225

357230.3550

T 0.6410

0.4971

357230.3550

$\mathrm{T} \quad 0.6410$

0.4971

357230.3550

$T \quad 0.6410$

0.4971

357230.3550

T 0.6410

0.4971

357230.3550

$\mathrm{T} \quad 0.6410$

0.4971

357230.3550

T 0.6410

0.4971

357240.4050

$T \quad 0.6770$

0.5426

(1)

0.6377

0.6377

0.6917

0.4068

0.3845

0.6917

0.4008

0.3783

0.6917

0.3913

0.3684

0.6917

0.3748

0.3513

0.6917

0.3712

0.3476

0.6917

0.3740

0.3529

0.6752
0.790 MICRON D. PARTICLES

(CONT INUED).

$0.36990 .4628 \quad 1.0000 E-040.3384$

$0.3652 \quad 0.4057600389 E-08 \quad 0.1529$

0.9219

0.35020 .2611

0.0039

$0.21540 .3131 \quad 1.0000 E-03 \quad 0.0844$

$0.1944 \quad 0.27375 .8513 E-08$ 0.1481

$0.2357 \quad 3.134$

0.0023

0.23690 .3131 1.0OOOE-03 0.0844

$0.21640 .2737 \quad 5.8513 E-08 \quad 0.1481$

$0.2357 \quad 3.134$

0.0023

0.2531 0.3131 1.0000E-03 0.0844

$0.23310 .2737508513 E-080.1481$

$\begin{array}{llll}0.6377 & 0.2357 & 3.134 & 0.0023\end{array}$

0.28170 .3131 1. OOOOE-03 0.0844

$0.26240 .27375 .8513 E-080.1481$

$\begin{array}{llll}0.6377 & 0.2357 & 3.134 & 0.0023\end{array}$

0.26230 .3008 1.2400E-03 0.0726

$0.24150 .2630 \quad 5.8402 E-080.1479$

$0.61270 .2264 \quad 3.931 \quad 0.0022$

$\begin{array}{lllll}0.2763 & 0.3008 & 1.2400 E-03 & 0.0726\end{array}$

$0.25590 .2630 \quad 5.8402 E-08 \quad 0.1479$

$0.6127 \quad 0.2264 \quad 3.931 \quad 0.0022$

0.28240 .3008 1.2400E-03 0.0726

$0.26220 .2630 \quad 508402 E-080.1479$

$0.6127 \quad 0.2264 \quad 3.931 \quad 0.0022$

$0.29090 .3008 \quad 1.2400 E-03 \quad 0.0726$

$0.2709 \quad 0.2630 \quad 5.8402 E-080.1479$

0.6127

$0.2264 \quad 3.931$

0.3962

0.3735

$0.44626 .7000 E-04$

0.0022

0.4055

0.3630

$5.6429 E-08$

0.0932

0.4462

2.660

0.1429

0.4055

0.3630

$6.7000 E-04$

0.0039

$5.6429 E-080.1429$

$2.660 \quad 0.0039$

0.4462

0.4055

$6.7000 E \div 04$

0.0932

$5.6429 E-08 \quad 0.1429$

2.660

0.0039

0.3630

6. 7000E-04

0.0932

0.4462

5.6429E-08

0.1429

$0.3630 \quad 2.660$

0.0039

0.4462

6.700OE-04

0.0932

0.4055

$5.6429 E-08$

0.1429

$0.3630 \quad 2.660$

0.0039

0.4462

6. $7000 E-04$

0.0932

0.4055

$5.6429 E-08$

0.1429

$0.3630 \quad 2.660$

0.0039

0.4018

8.3000E-04

0.0834

0.3605

0.3185

5.7160E-08

0.1447

3.019

0.0033
REI

RED

RELM

$$
\text { 668. 5.4010E-03 }
$$

359.

438 .

6529.2.1651E-02

4471 .

5260 .

6529.2.1651E-02

4471 .

5260 。

5529, 2.1651E-02

4471 。

5260 。

6529.2.1651E-02

4471 .

5260 。

80960

5642 .

6600 。

8096.

5642 .

6600 。

8096 .

5642 .

6600 .

8096.

5642 。

6600 。

6340.1.9605E-02

3462 。

4482 。

$6340.1 .9605 E-02$

3462。

4482 。

6340. 1.9605E-02

3462 。

4482 。

6340 。

3462 。

4482 。

6340 。

3462 。

4482 。

6340 .

3462 。

4482 。

6853 .

4060 .

5077 。
2. $5159 E-02$

2. 5159E-02

$2.5159 E-02$

$2.5159 E-02$

1.9605E-02

1. $9605 E-02$

1. $9605 E-02$

2. $1918 E-02$ 
N2 - $\mathrm{NH} 3$

, 0.790

MICRON

N $D$

PARTICLES (CONTI VUED).

$\begin{array}{llllllll}\text { RUN } & \text { YINTI } & \text { EPEXP } & \text { EPMO } & \text { QINT } & \text { FILMR } & \text { REI } & \text { MTC } \\ \text { TYPE } & \text { YINTO } & \text { EPCORR } & \text { EPSW } & \text { PLM } & \text { KN } & \text { RED } & \\ & \text { YINTLM } & \text { EMT } & \text { EPMA } & \text { ULM } & \text { UR } & \text { RELM } & \\ 35724 & 0.4050 & 0.3749 & 0.4018 & 8.3000 E-04 & 0.0834 & 6853 . & 2.19185-02 \\ T & 0.6770 & 0.3539 & 0.3605 & 5.7160 E-08 & 0.1447 & 4060 . & \\ & 0.5426 & 0.6752 & 0.3185 & 3.019 & 0.0033 & 5077 . & \\ 35724 & 0.4050 & 0.3545 & 0.4018 & 8.3000 E-04 & 0.0834 & 6853 . & 2.1918 E-02 \\ T & 0.6770 & 0.3328 & 0.3605 & 5.7160 E-08 & 0.1447 & 4060 . & \\ & 0.5426 & 0.6752 & 0.3185 & 3.019 & 0.0033 & 5077 . & \end{array}$


TABLE XVII

DATA FOR N2

- $\mathrm{NH3}$

1. 1.011 MICRON DI AMETER PARTICLES。

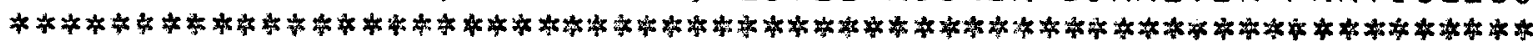

INERT GAS FLOW RATE CONSTANT. IN ALL RUNS.

INLET TEMP。(DEG。K) 293.0 , PRESSURE (ATM。) 1.000

CALIBRATION CORRECTION: COUNT/TRUE COUNT

INLET: $\quad 1.0000+0.0$ YINTI + 0.0 *YTRNI/YINTI

OUTLET: $1.0000+0.0$ *INTO + 0.0350*YTRNO/YIVTJ

GAS

N2

(INERT)

MOLEC。 WEIGHT (G/GMOLE)

DENSITY (KG/M* * 3 )

VISCOSITY (KG/M/SEC)

MEAN FREE PATH (M)

VOLUME* $(1 / 3)$ (M/GMOLE* $(1 / 3))$

$\begin{array}{ll}28.02 & 17.03 \\ 1.165 & 0.7182 \\ 1.7480 E-05 & 9.8200 E-06 \\ 6.3900 E-08 & 4.5700 E-08 \\ 3.1190 E-02 & 2.2240 E-02\end{array}$

2. $3212 E-05$

$(M * 2 / S E C)$

$\begin{array}{llllllll}\text { RUN } & \text { YINTI } & \text { EPEXP } & \text { EPMO } & \text { QINT } & \text { FILMR } & \text { REI } & \text { MTC } \\ \text { TYPE } & \text { YINTO } & \text { EPCORR } & \text { EPSW } & \text { PLM } & \text { KN } & \text { REO } & \\ & \text { YINTLM } & \text { EMT } & \text { EPMA } & \text { ULM } & \text { UR } & \text { RELM }\end{array}$

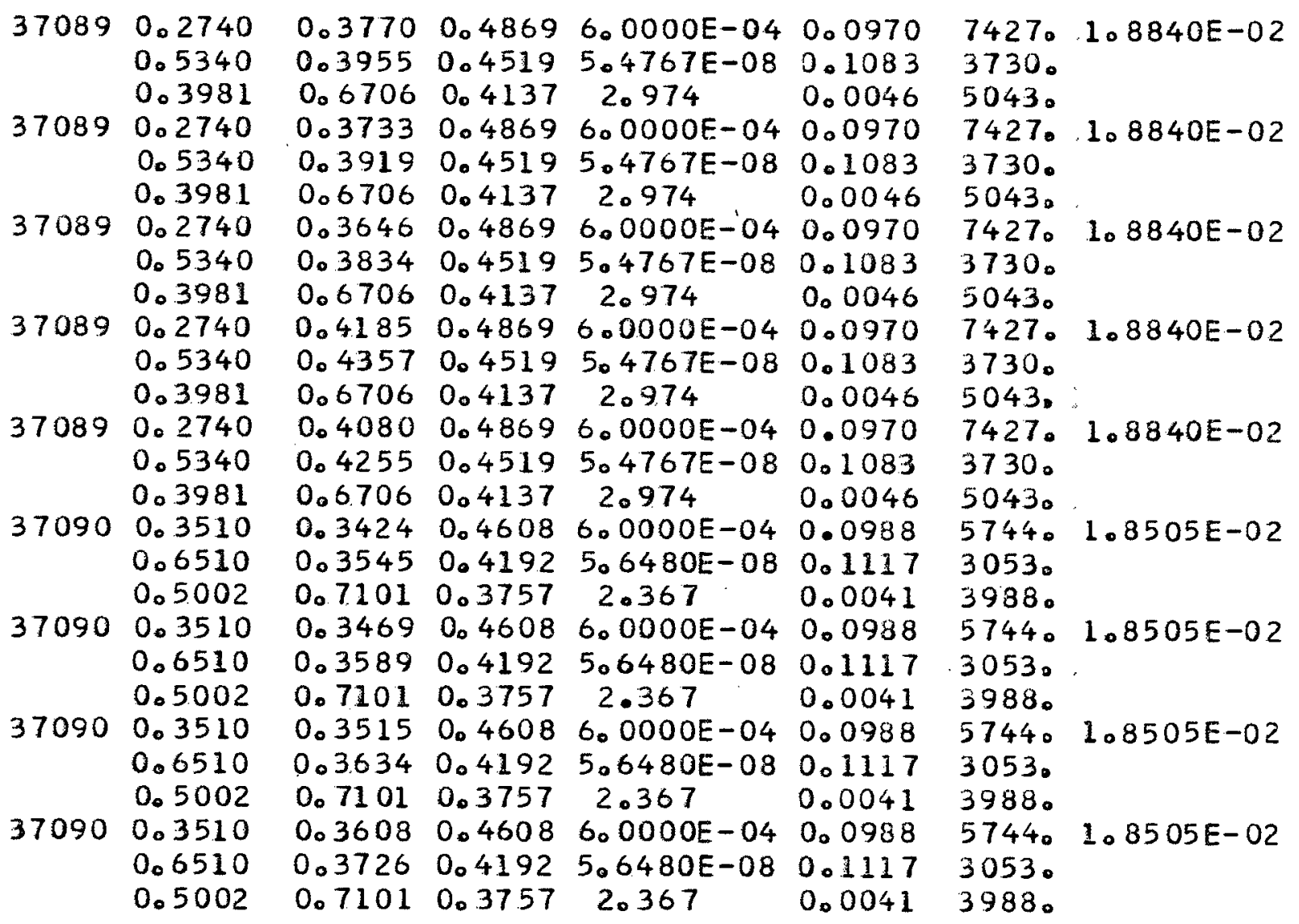


N2 - NH3
-

1.011 MICRON J。
PARTICLES

QINT

PLM

ULM

FILMR
KN
UR
0.7160

0.5708

370910.4180

0.7160

0.5708

370910.4180

0.7160

0.5708

370920.5040

0.7860

0.6538

370920.5040

0.7860

0.6538

370920.5040

0.7860

0.6538

$37093 \quad 0.6300$

0.8560

0.7547

370930.6300

0.8560

0. 7547

$37093 \quad 0.6300$

0.8560

0.7547

370940.7600

0.9140

0.8476

370940.7600

0.9140

0.8476

370940.7600

0. 9140

0.8476

370950.8750

0.9590

0.9240

$37095 \quad 0.8750$

0.9590

0.9240

370950.8750

0.9590

0.9240

370950.8750

0.9590

0.9240
0.1054 0.1140

$0.30890 .41626 .0000 E-04$

$0.31840 .3722 \quad 5.7603 E-08$

$0.71510 .3278 \quad 2.075$

0.3027

0.4162

6.075

0.3122

0

0.3107

0.3722

0.0034

0.3201

0,4162

5.7603E-08

2. 075

0.1054

0.1140

0.0034

0.7151

$0.37225 .7603 E-08$

$0.3278 \quad 2.075$

0.2588

0.2658

0.7233

0.2772

0.2840

0.7233

0.2789

0.2857

0.7233

0.1924

0.1971

0.7136

0.1985

0.2032

0.7136

0.3588

6.0000E-04

$0.3140 \quad 5.8877 \mathrm{E}-08$

$0.2707 \quad 1.811$

0.1054

0.1140

0.0034

0.1117

0.1165

0.0027

$0.35886 .0000 E-04$

0.1117

0.3140 5. $8877 E-08$

0.1165

$0.2707 \quad 1.811$

0.0027

0.3588 6.0000E-04

0.1117

$0.3140 \quad 5.8877 \mathrm{E}-08$

$0.2707 \quad 1.811$

0.1165

0.0027

$0.26406 .0000 E-04$

0.1257

$0.22416 .0373 E-08$

0.1877

1.569

0.1194

0.0018

$0.2640 \quad 6.0000 E-040.1257$

0.2241

0.1877

6.0373E-08

1.569

0.1194

0. 1952

0.1999

0.2640

0.2241

0.7136

0.0847

0.0877

0.7020

0.1877

6.0000E-04

0.0018

6.0373E-08

1.569

0.1257

0.1685

0.1387

0.1130

6.0000E-04

0.1194

0.0018

$6.1718 E-08$

1.397

0.1396

0.1221

0.0010

0.0787

0.0817

0.1685

0.1387

6. 0000E-04

0.1396

0.7 .020

0.1130

$6.1718 E-08$

1.397

0.1221

0.0010

0.0836

0.0866

0.7020

0.1685

6.0000E-04

0.1396

0.0352

0.1387

0.1130

.1718E-08

1.397

0.1221

0.0010

0.0366

0.7007

0.0417

0.0431

0.7007

0.0069

0.0084

0.7007

0.0504

0.0518

0.7007
0.0876

D. 0702

0.0560

0.0876

0.0702

0.0560

0.0876

0.0702

0.0560

0.1478

6.2814E-08 0.1243

1.281

0.0005

6.00DOE-04 0.1478

$6.2814 E-08 \quad 0.1243$

1.281

0.0005

6.0000E-04 0.1478

6. $2814 E-08$ 0.1243

1.281

0.0005

6. $0000 E-04 \quad 0.1478$

0.0702

6.2814E-08

1.281
0.1243

0.0005
6.0000E-04
(CONTINUED)。

REI
RED
RELM

MTC

4795。1.7339E-02

2776 。

3486 。

4795.1.7339E-02

2776 。

3486 。

4795. 1. $7339 E-02$

2776 。

3486 .

3957.1.6358E-0.2

2531 。

3039 。

3957.1.6358E-02

2531 。

3039 。

3957.1.6358E-02

2531 。

30390

3155。1.4539E-02

2328.

2635 。

3155.1.4539E-02

2328 .

2635 .

3155 。

2328.

2635 。

2616. 1.3091E-02

2185.

$2351 。$

2616. 1. $3091 \mathrm{E}-02$

2185.

2351 。

2616.1.3091E-02

2185 。

2351 。

2279. 1.2369E-02

2087 。

2162 .

2279。1.2369E-02

2087 。

2162 。

2279.1.2369E-02

2087。

2162 。

2279. 1.2369E-02

2087 .

2162 . 
N2 - $\mathrm{NH} 3$

RUN

\section{YINTI}

YINTO

YINTLM

$37095 \quad 0.8750$

0.9590

0.9240

$37095 \quad 0.8750$

0.9590

0.9240

370950.8750

0.9590

0.9240

$37100 \quad 0.2740$

0.5340

0.3981

$37100 \quad 0.2740$

0. 5340

0.3981

$37100 \quad 0.2740$

0. 5340

0.3981

$37100 \quad 0.2740$

0.5340

0.3981

386510.3510

0.6510

0.5002

38651 0.3510

0.6510

0.5.002

386510.3510

0.6510

0.5002

386510.3510

0.6510

0.5002

386520.4180

0.7160

0.5708

386520.4180

0.7160

0.5708

386520.4180

0. 7160

0.5708

1

\section{EPEXP}

EPCORR

EMT

0.1212

0.1225

0.7007

0.0926

0.0940

0.7007

0.0701

0.0715

0.7007

0.3827

0.4010

0.6706

0.3708

0.3894

0.6706

0.3746

0.3931

0.6706

0.3685

0.3872

0.6706

0.3685

0.3801

0.7101

0.3519

0.3638

0.7101

0.3398

0.3520

0.7101

0.3509

0.3629

0.7101

0.2971

0.3067

0.7151

0.3098

0.3193

0.7151

0.3155

0.3249

0.7151

\section{EPMO}

EPSW

EPMA

0.0876

0.0702

0.0560

0.0876

0.0702

0.0560

0.0876

0.0702

0.0560

0.4869

0.4519

0.4137

0.4869

0.451

0.4137

0.4869

0.4519

0.4137

0.4869

0.4519

0.4137

0.4608

0.4192

0.3757

0.4608

0.4608

0.4192

0.3757

0.460

0.419

0.3757

0.4608

0.419

0.3757

0.4162

0.3722

0.3278

0.4162

0.3722

0.3278

0.4162

0.3722

0.3278

QINT
PLM
ULM

PARTICLES

6. $0000 E-04$

6. $2814 E-08$

1.281

6.0000E-04

$6.2814 E-08$

1.281

6.0000E-04

$6.2814 E-08$

1. 281

6.0000E-04

$5.4767 E-08$

2. 974

6.0000E-04

$5.4767 E-08$

2.974

6.0000E-04

$5.4767 E-08$

2. 974

6.0000E-04

$5.4767 E-08$

2.974

6.0000E-04

5.6480E-08

2.367

6.0000E-04

5.6480E-08

2.367

6. $0000 E-04$

5.6480E-08

2.367

6. 0000E-04

5.6480E-08

2.367

6. OOODE-04

$5.7603 E-08$

2. 075

6. DOOOE-04

$5.7603 E-08$

2. 075

6.0OOOE-04

5. $7603 E-08$

2. 075
FIL YR

$K N$

UR

0.1478

0.1243

0.0005

0.1478

0.1243

0.0005

0. 1478

0.1243

0.0005

0.0970

0.1083

0.0046

0.0970

0.1083

0.0046

0.0970

0.1083

0.0046

0.0970

0.1083

0.0046

0.0988

0.1117

0.0041

0.0988

0.1117

0.0041

0.0988

0.1117

0.0041

0.0988

0.1117

0.0041

0. 1054

0.1140

0.0034

0.1054

0.1140

0.0034

0.1054

0.1140

0.0034
(CONTINUED)。

REI MTC

REO

२ELM

2279.1.2369E-02

2087 .

2162 。

2279.1.2369E-02

2087 。

2162 。

2279. 1.2369E-02

2087.

2162 。

7427。, 1.8840E-02

3730 。

5043 .

7427.,1.8840E-02

3730 。

5043 。

7427。1.8840E-02

3730 。

5043.

7427 .

3730 。

5043。

5744。1.8505E-02

3053 。

3988 。

5744。1.8505E-02

3053 。

3988 。

$5744.1 .8505 E-02$

3053 。

3988 。

5744. 1.8505E-02

3053 。

3988 .

4795。1.7339E-02

2776 。

3486 。

4795。.1.7339E-02

2776 。

3486 。

4795. 1.7339E-02

2776 。

3486 。 
TABLE XVIII

DATA FOR N2

, $\mathrm{NH} 3$

- 2.020 MICRON DI AMETER PART ICLES-

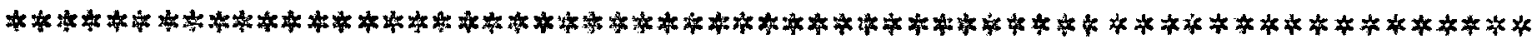

SERIES 1.

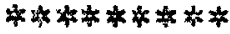

INERT GAS FLOW RATE CONSTANT IN ALL RUNS。

INLET TEMP。 IDEG。K) 293.0 , PRESSURE (ATM。) 1.000

CALIBRATION CORRECTION: COUNT/TRUE COUNT

INLET: $\quad 1.0000+0.0$ *INTI + 0.0 *YTRNI/YINTI

OUTLET: $1.0000+0.0$ *INTO + 0.0 *YTRNO/YINTO

GAS

$\begin{array}{ll}\text { MOLEC。 WEIGHT } & \text { (G/GMOLE) } \\ \text { DENSITY } & \text { (KG/M**3) } \\ \text { VISCOSITY } & (K G / M / S E C) \\ \text { MEAN FREE PATH } & (M) \\ \text { VOLUME* }(1 / 3) & (M / G M O L E * * 11 / 3)) \\ \text { DIFFUSIVITY } & (M * 2 / S E C)\end{array}$

$\begin{array}{llllllll}\text { RUN } & \text { YINTI } & \text { EPEXP } & \text { EPMD } & \text { QINT } & \text { FILMR } & \text { REI } & \text { MTC } \\ \text { TYPE } & \text { YINTO } & \text { EPCORR } & \text { EPSW } & \text { PLM } & \text { KN } & \text { RED } & \\ & \text { YINTLM } & \text { EMT } & \text { EPMA } & \text { ULM } & \text { UR } & \text { RELM }\end{array}$

344140.6300

0.8560

0.7547

0.1982

0.1982

0.7136

344140.6300

0.8560

0.7547

0.2247

0.2247

0.7136

344140.6300

0.8560

0. 7547

0.1690

0.1690

0.7136

344140.6300

0.8560

0.7547

0.2294

0.2294

0.7136

344140.6300

0.8560

0.7547

0.1985

0.1985

0.7136

344160.5040

0.7860

0.6538

0.3451

0.3451

0.7233

344160.5 .040

0.3081

0.7860

0.6538

0.3081

0.7233

344160.5040

0.7860

0.6538

344160.5040

0.2985

0.2985

0.7233

0.3142

0.7860

0.6538
N2

(INERT)

$$
\begin{aligned}
& 28.02 \\
& 1.165 \\
& 1.7480 E-05 \\
& 6.3900 E-08 \\
& 3.1190 E-02
\end{aligned}
$$

NH3

(TRANSFERRED)

$$
\begin{aligned}
& 17.03 \\
& 0.7182 \\
& 9.8200 E-06 \\
& 4.5700 E-08 \\
& 2.2240 E-02
\end{aligned}
$$

\section{$2.3212 E-05$}

RELM

3155。1.4539E-02

2328 。

2635 .

3155.1.4539E-02

2328 。

2635 。

3155。1.4539E-02

2328 。

2635 。

3155。1.4539E-02

2328 。

2635 .

3155, 1.4539E-02

2328 .

2635 。

3957.1.6358E-02

2531 。

3039 。

3957.1.6358E-02

2531 。

3039 .

3957.1.6358E-02

2531 。

3039 。

3957.1.6358E-02

2531。

3039 。 
N2 - $\mathrm{NH3}$

RUN YINTI YINTO YINTLM

, 2.020 MICRON D. PARTICLES (CDNT I NUED).

\section{TYPE}

0. 4180

0.7160

0.5708

344170.4180

0.7160

0.5708

344170.4180

0.7160

0.5708

344180.2740

0.5340

0.3981

344180.2740

0.5340

0.3981

$34420 \quad 0.3050$

0.5900

0.4433

$34420 \quad 0.3050$

0.5900

0.4433

$34420 \quad 0.3050$

0.5900

0.4433

344210.3510

0.6510

0.5002

344210.3510

0.6510

0.5002

344210.3510

0.6510

0.5 .002

344210.3510

0.6510

0.5002

344210.3510

0.6510

0.5002
EPEXP EPCORR EMT

0.37480 .41626

0.37480 .3722

0.71510 .3278

0.39730 .4162

0.39730 .3722

0.7 .1510 .3278

$0.3857 \quad 0.4162$

0.38570 .3722

$\begin{array}{lll}0.7151 & 0.3278\end{array}$

0.5043

0.5043

0.6706

0.4869

0.4519

0.4137

0.5051

0.5051

0.6706

0.4776

0.4776

0.6950

0.4869

0.4519

0.4137

0.4831

0.4447

0.4036

0.4821

0.4821

0.4831

0.6950

0.4447

0.4823

0.4823

0.4036

QINT
PLM
ULM

FILMR

KN

UR

$6.0000 E-040.1054$

5. $7603 E-08$

0.0570

2.075

0.0034

6.0000E-04 0.1054

5. $7603 E-08$

0.0570

2.0 .75

0.0034

6. $0000 E-04 \quad 0.1054$

5. $7603 E-08$

0.0570

2.075

0.0034

6. $0000 E-04$

0.0970

5.4767E-08

0.0542

2.974

0.0046

6. 0000E-04

0.0970

$5.4767 E-08$

0.0542

2.974

0.0046

6.000OE-04

0.0961

$5.5541 E-08$

0.0550

2.671

0.0045

6. OOOOE-04 0.0961

$5.5541 E-08$

0.0550

2.671

0.0045

0.4831

6.00OOE-04

0.0961

0.6950

0.4447

$5.5541 E-08$

0.0550

0.3936

0.3936

0.4036

2.671

0.0045

$0.46086 .0000 E-040.0988$

0.4192 5.6480E-08

0.0559

0.7101

0.3855

0.3855

0.3757

2.367

0.0041

0.4608 6.0000E-04 0.0988

0.7101

0.4192

$506480 E-080$

0.0559

$0.3757 \quad 2.367$

0.0041

0.3917

0.3917

0.7101

0.4608

6. OOOOE-04

0.0988

0.4192 5.6480E-08 0.0559

$0.3757 \quad 2.367$

0.0041

0.4178

0.4178

0.4608

6.0000E-04

0.0988

0.7101

0.4192

5.

$06480 E-08$

0.0559

0.4325

0.37572 .367

0.0041

0.4325

0.4608

6. $0000 E-04$

0.0988

0.7101

0.4192

$5.6480 E-08$

0.0559

0.0041
REI

REO

RELM

4795. 1.7339E-02

2776 。

3486 。

4795. 1.7339E-02

2776.

3486 。

$4795 \circ 1.7339 \varepsilon-02$

2776 。

3486 .

$7427.1 .8840 E-02$

3730 。

50430

7427。1.884OE-02

3730 。

5043 。

6645. 1.9013E-02

3371 。

4513 。

6645 。

3371 。

4513 。

6645 .

3371 。

4513 。

5744.1.8505E-02

3053 。

3988 .

5744.1.8505E-02

3053 。

3988 。

5744。 1.8505E-02

3053 。

3988 。

5744。1.8505E-02

3053 。

3988 。

5744。1.8505E-02

3053 .

3988 . 
TABLE XIX

DATA FOR N2 , NH3 2.020 MICRON DIAMETER PARTICLES-

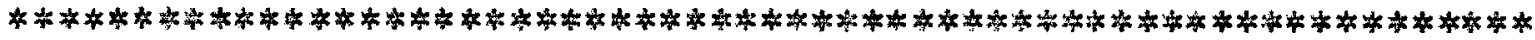
SERIES 2 。

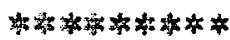

INERT GAS FLOW RATE CONSTANT IN ALL RUNS。

INLET TEMP。 (DEG。 K) 293.0 , PRESSURE (ATM。). 1.000

CALIBRATION CORRECTION: COUNT/TRUE COUNT

INLET: $\quad 1.0000+0.0$ *YINTI + 0.0 *YTRNI/YINTI

OUTLET: $0.8550+0.1450$ *YINTO + 0.0 *YTRNOIYINTJ

GAS

N2

(INERT)

MOLEC。WEIGHT

DENSITY

VISCOSITY

MEAN FREE PATH

VOLUME $\$(1 / 3)$

DIFFUS IVITY

$(M * * 2 / S E C)$

(G/GMOLE)

$(K G / M * 3)$

$(K G / M / S E C)$

(M)

(M/GMOLE* $(1 / 3))$

$\begin{array}{ll}28.02 & 17.03 \\ 1.165 & 0.7182 \\ 1.7480 E-05 & 9.8200 E-06 \\ 6.3900 E-08 & 4.5700 E-08 \\ 3.1190 E-02 & 2.2240 E-02\end{array}$

$2.3212 E-05$

$\begin{array}{llllllll}\text { RUN } & \text { YINTI } & \text { EPEXP } & \text { EPMO } & \text { QINT } & \text { FILMR } & \text { REI } & \text { MTC } \\ \text { TYPE } & \text { YINTO } & \text { EPCORR } & \text { EPSW } & \text { PLM } & \text { KN } & \text { REO } & \\ & \text { YINTLM } & \text { EMT } & \text { EPMA } & \text { ULM } & \text { UR } & \text { RELM }\end{array}$

$34436 \quad 0.2740$

0.5340

0.5283

0.4869

6. ODOOE- 04

0.0970

0.4941

0.45195

$5.4767 E-08$

0.0542

0.3981

0.6706

$0.4137 \quad 2.974$

0.0046

$0.5100 \quad 0.4869 \quad 6.0000 E-040.0970$

0.4745

$0.45195 .4767 \mathrm{E}-08$

0.0542

0.3981

0.6706

$0.4137 \quad 2.974$

0.0046

344360.2740

0.5134

$0.48696 .0000 E-04$

0.0970

0.5340

0.4781

$0.45195 .4767 E-08$

0.0542

0.6706

$0.4137 \quad 2.974$

0.0046

344420.3050

0.4958

0.4831

$6.0000 E-04$

0.0961

0.4639

$0.44475 .5541 E-08$

0.0550

0.5900

0.6950

$0.4036 \quad 2.671$

0.0045

344420.3050

0.4739

0.4831

6.0000E-04

0.0961

0.5900

0.4406

0.4447

5.5541E-08

0.0550

0.4433

0.6950

$0.4036 \quad 2.671$

0.0045

344420.3050

0.4712

0.4831

6.0000E-04

0.0961

0.5900

0.4378

0.4447 5.5541E-08

0.0550

0.4433

0.6950

$0.4036 \quad 2.671$

0.0045

344380.3510

0.4285

0.4036

20671

0.0988

0.6510

0.5002

0.3980

.4608 6.0000E-04

0.0559

0.7101

0.4192 5.6480E-08

0.0041

344380.3510

0.4534

$0.3757 \quad 2.367$

0.0988

0.6510

0.5002

0.4243

0.7101

0.4608 6.0000E-04

0.0559

$\begin{array}{rr}0.4192 & 5.6480 \\ 0.3757 & 2.367\end{array}$

0.0041

344380.3510

0.4535

0.460

0.0988

0.6510

0.5002

0.4244

$6.0000 E-04$

0.0559

0.7101

$0.3757 \quad 2.367$

0.0041
7427.

1. $8840 E-02$

3730 .

5043 。

7427 。

3730 .

NH3

5043 。

7427。.1.8840E-02

3730 。

5043 。

6645. 1.9013E-02

3371 。

4513 .

6645.1.9013E-02

3371 。

4513 。

$6645 \circ 1.9013 E-02$

3371 。

4513 。

5744。1.8505E-02

3053 。

3988 .

5744०1.8505E-02

3053.

3988 。

5744. 1.8505E-02

3053 .

3988 。 
N2 - $\mathrm{NH3}$
,

$\begin{array}{ll}\text { RUN } & \text { YINTI } \\ \text { TYPE } & \text { YINTO } \\ & \text { YINTLM } \\ 34438 & 0.3510 \\ & 0.6510 \\ & 0.5002 \\ 34439 & 0.4180 \\ & 0.7160 \\ 34439 & 0.5708 \\ & 0.4180 \\ & 0.7160 \\ & 0.5708\end{array}$

344390.4180

0.7160

0.5708

$34440 \quad 0.5040$

0.7860

0.6538

$34440 \quad 0.5040$

0.7860

0.6538

$34440 \quad 0.5040$

0.7860

0.6538

344410.6300

0.8560

0.7547

344410.6300

0.8560

0.7547

344410.6300

0.8560

0.7547

344430.7600

0. 9140

0.8476

344430.7600

0.9140

0.8476

344430.7600

0.9140

0.8476

344430.7600

0.9140

0. 8476

$34444 \quad 0.8750$

0.9590

0.9240

344440.8750

0.9590

0. 9240
EPEXP

EPCORR

EMT

0.4502

0.4209

0.7101

0.3928

0.3667

0.7151

0.3978

0.3719

0.7151

0.4090

0.3836

0.7151

0.2779

0. 2548

0.7233

0.3288

0.3073

0.7233

0.3536

0.3329

0.7233

0.2304

0.2140

0.7136

0.2346

0.2183

0.7136

0.2434

0.2273

0.7136

0.1393

0.1284

0.7020

0.1443

0.1335

0.7020

0.1488

0.1381

0.7020

0.1796

0.1692

0.7020

0.0651

0.0595

0.7007

0.0520

0.0463

0.7007

2.020 MICRON D.

PARTICLES

EPMO
EPSW
EPMA

0.4608

0.4192

0.3757

0.4262

0.3722

0.3278

0.4162

0.3722

0.3278

0.4162

0.3722

0.3278

0.3588

0. 3140

0.2707

0.3588

0.3140

0.2707

0.3588

0.3140

0.2707

0.2640

0.2241

0.1877

0.2640

0.2241

0.1877

0.2640

0.2241

0. 1877

0.1685

0.1387

0.1130

0.1685

0.1387

0.1130

0.1685

0.1387

0.1130

0.1685

0. 1387

0.1130

0.0876

0.0702

0.0560

0.0876

0.0702

0.0560

QINT
PLM
ULM

FILMR

KN

UR

6.0000E-04 0.0988

$5.6480 E-08$

2.367

0.0559

0.0041

6.0000E-04 0.1054

$5.7603 E-08$

2.075

0.0570

0.0034

6.0000E-04 0.1054

5.7603E-08

2.075

0.0570

0.0034

6.0000E-04 0.1054

5. $7603 E-08$

2. 075

6.0000E-04

5. $8877 \mathrm{E}-08$

1.811

6.0000E-04

5. $8877 E-08$

1.811

6.000OE-04

5. $8877 E-08$

1.811

6. OOODE-04

$6.0373 E-08$

1.569

6.0000E-04

$6.0373 E-08$

1.569

6. $0000 E-04$

$6.0373 E-08$

1.569

6.0000E-04

$6.1718 E-08$

1.397

0.0570

0.0034

0.1117

0.0583

0.0027

0.1117

0.0583

0.0027

0.1117

0.0583

0.0027

0.1257

0.0598

0.0018

0.1257

0.0598

0.0018

0.1257

0.0598

0.0018

0.1396

0.0611

0,0010

6.0000E-04 0.1396

6. $1718 \mathrm{E}-08$

1.397

0.0611

0.0010

6.0000E-04 0.1396

6.1718E-08 0.0611

1.397

0.0010

6.0000E-04 0.1396

6. $1718 E-08$

1.397

0.0611

0.0010

6.000OE-04 0.1478

6. $2814 E-08$

0.0622

1.281

0.0005

6.0000E-04

$6.2814 E-08$

1.281
0.1478

0.0622

0.0005

(CONTINUED).

REI
REO
RELM

MTC

5744。1.8505E-02

3053 .

3988 。

4795, 1.7339E-02

2776 。

3486 。

4795. 1. $7339 E-02$

2776.

3486 。

4795 .

2776 。

3486.

$3957.1 .6358 E-02$

2531 。

3039.

$3957.1 .6358 E-02$

2531 。

3039 。

3957.1.6358E-02

2531 。

3039 .

3155.1.4539E-02

2328 。

2635 。

3155.1.4539E-02

2328 。

2635 。

3155,1.4539E-02

2328 .

2635 。

2616.1.3091E-02

2185.

2351 。

2616.1.3091E-02

2185 。

2351 。

2616 .

2185 。

2351 。

2616 。

2185 。

2351 。

2279 。

2087 。 
N2 , NH3 - 2.02

\begin{tabular}{|c|c|c|c|c|c|c|c|}
\hline $\begin{array}{l}\text { RUN } \\
\text { TYPE }\end{array}$ & $\begin{array}{l}\text { YINTI } \\
\text { YINTO } \\
\text { YINTLM }\end{array}$ & $\begin{array}{l}\text { EPEXP } \\
\text { EPCORR } \\
\text { EMT }\end{array}$ & $\begin{array}{l}\text { EPMO } \\
\text { EPSW } \\
\text { EPMA }\end{array}$ & $\begin{array}{l}\text { QINT } \\
\text { PLM } \\
\text { ULM }\end{array}$ & $\begin{array}{l}\text { FILMR } \\
\text { KN } \\
\text { UR }\end{array}$ & $\begin{array}{l}\text { REI } \\
\text { REO } \\
\text { RELM }\end{array}$ & MTC \\
\hline $\begin{array}{l}34444 \\
34444\end{array}$ & $\begin{array}{l}0.8750 \\
0.9590 \\
0.9240 \\
0.8750 \\
0.9590 \\
0.9240\end{array}$ & $\begin{array}{l}0.0842 \\
0.0787 \\
0.7007 \\
0.1152 \\
0.1099 \\
0.7007\end{array}$ & $\begin{array}{l}0.0876 \\
0.0702 \\
0.0560 \\
0.0876 \\
0.0702 \\
0.0560\end{array}$ & $\begin{array}{l}6.0000 E-04 \\
6.2814 E-08 \\
1.281 \\
6.0000 E-04 \\
6.2814 E-08 \\
1.281\end{array}$ & $\begin{array}{l}0.1478 \\
0.0622 \\
0.0005 \\
0.1478 \\
0.0622 \\
0.0005\end{array}$ & $\begin{array}{l}2279 \circ \\
2087^{\circ} \\
21622^{\circ} \\
2279^{\circ} \\
2087^{\circ} \\
2162 .\end{array}$ & $1.2369 E-02$ \\
\hline
\end{tabular}


TABLE XX

DATA FOR AR NH3 , 0.790 MICRDN DIAMETER PARTICLES。

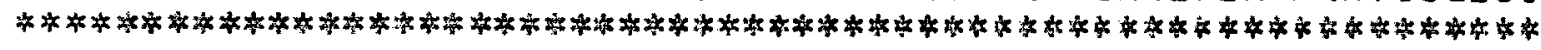

INERT GAS FLDW RATE CONSTANT IN ALL RUNS。

INLET TEMP。 (DEG。 K) 293.0 , PRESSURE (ATM。) 1.000

CAL I BRATION CORRECTION: COUNT/TRUE CDUNT

INLET: $1.0000+0.0$ \#YINTI +0.0 *YTRNI/YIVTI

DUTLET: $1.0000+0.0$ \$YINTO + 0.0 *YTRNO/YINTJ

GAS

AR
(INERT)

39.91
1.661
$2.2170 E-05$
$6.7900 E-08$
$2.8970 E-02$

$\mathrm{NH} 3$

(TRANSFERRED)

$\begin{array}{ll}\text { MOLEC。 WEIGHT } & \text { (G/GMOLE) } \\ \text { DENSITY } & (K G / M * 3) \\ \text { VISCDSITY } & (K G / M / S E C) \\ \text { MEAN FREE PATH } & (M) \\ \text { VOLUME**(1/3) } & (M / G M O L E * *(1 / 3))\end{array}$

DIFFUSIVITY $(M * 2 / S E C)$

$\begin{array}{llllllll}\text { RUN } & \text { YINTI } & \text { EPEXP } & \text { EPMO } & \text { QINT } & \text { FILMR } & \text { REI } & \text { MTC } \\ \text { TYPE } & \text { YINTO } & \text { EPCORR } & \text { EPSW } & \text { PLM } & \text { KN } & \text { RED } & \\ & Y I N T L M & \text { EMT } & \text { EPMA } & \text { ULM } & \text { UR } & \text { RELM }\end{array}$

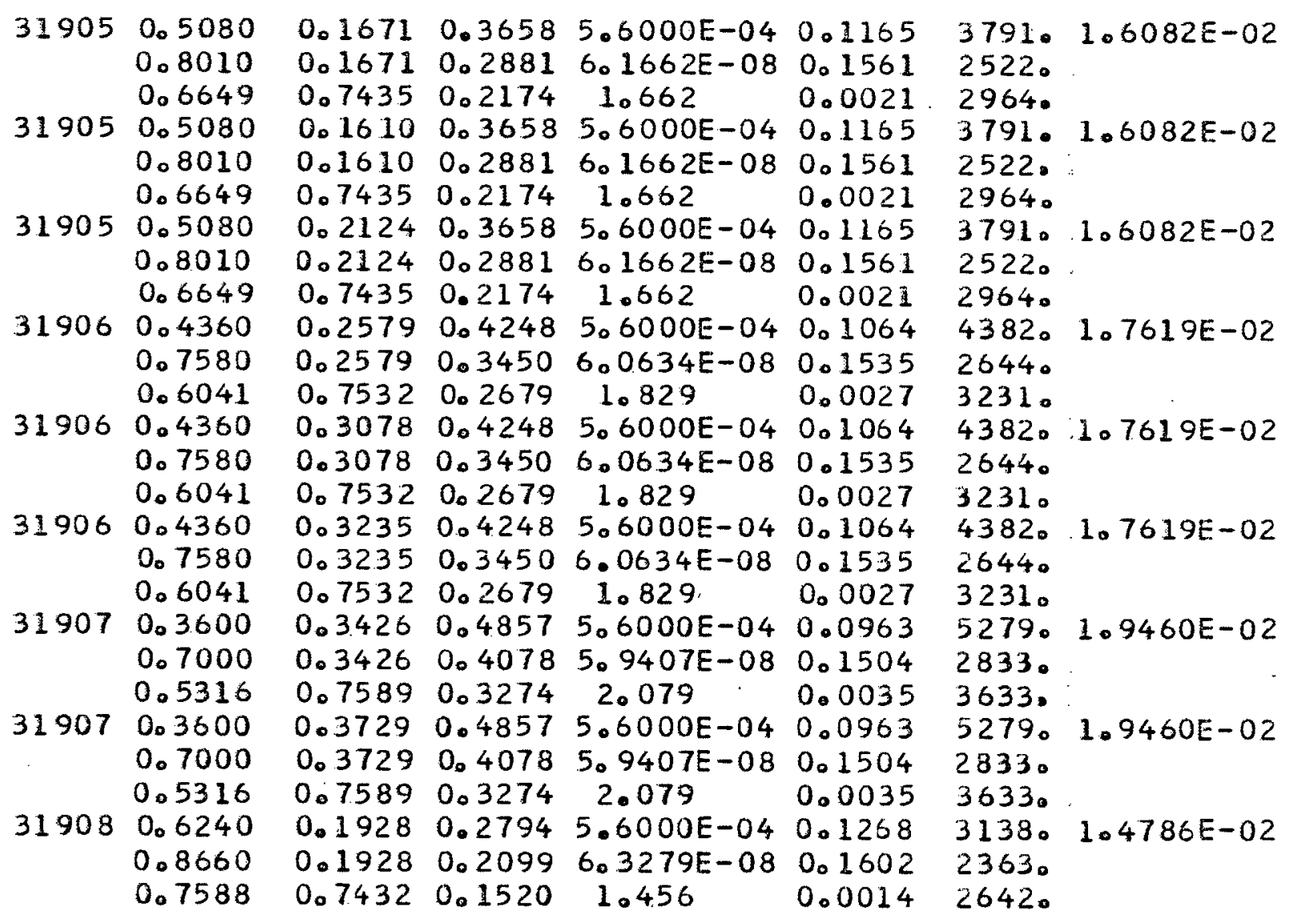


$A R$ - $\mathrm{NH3}$

RUN Y INT I

TYPE YINTO Y INTLM

$31908 \quad 0.6240$ 0.8660 0.7588

319090.7960 0.9260 0.8702

319090.7960 0.9260 0. 8702

319090.7960 0.9260 0. 8702

319120.3600 0.7 .000 0.5316

319130.3600 0.7000 0.5316

319130.3600 0.7000 0.5316

319140.4360 0.7580 0.6041

319140.4360 0.7580 0.6041

319150.5080 0.8010 0.6649

319160.6240 0.8660 0.7588

319160.6240 0.8660 0. 7588

319180.4360 0.7580 0.6041

319180.4360 0.7580 0.6041

319190.3600 0.7000 0.5316

319190.3600 0.7000 0.5316

,

0.790 MICRON D。 PARTICLES

EPEXP

EPMO

EPCORR EPSW

EMT

EPMA

0.1845

0.1845

0.2794

0.2099

0.7432

0.1520

QINT
PLM
ULM

F ILMR

$K N$

UR

\subsection{9}

0.0669

0.6882

0.1404

0.0987

0.0678

0.0564

0.0564

0.6882

0.1404

0.0987

0.0678

$5.6000 E-04$

$6.3279 E-08$

1.456

0.1268

0.1602

0.0474

0.0474

0.6882

0.1404

0.0 .987

$5.6000 E-04$

0.1621

0.3761

0.0678

. 5304E-08

1.270

0.1653

5. 6000E-04

0.1621

0.3761

0.7589

0.4857

$.5304 E-08$

0.1653

1.270

0.0006

0.3088

0.4078

5.6000E-04

0.1621

$.5304 E-08$

0.1653

1.270

0.0006

0.3088

0.4857

5. $6000 E-04$

0.0963

0.7589

0.4078

$.9407 E-08$

0.1504

0.0035

0.3253

0.3274

5. $6000 E-04$

0.0963

0.3253

0.4857

$5.9407 E-08$

0.1504

0.0035

0.7589

0.4078

2.079

0.3274

0.3395

0.4248

0.3395

0.3450

0.7532

0.3201

0.2679

5.6000E-04

0.0963

0.3201

0.4248

$.9407 E-08$

0.1504

2.079

0.0035

5.6000E-04

0.1064

6. $0634 E-08$

0.1535

0.0027

0.7532

0.3450

1.829

5.6000E-04

$6.0634 E-08$

0.1064

0.1535

0.2510

0.2679

1.829

0.0027

0.2510

0.7435

$0.36585 .6000 E-04$

$0.28816 .1662 E-08$

$0.2174 \quad 1.662$

0.1165

0.1561

0.0021

0.1481 0.2794

0.1481

0.2099

5. $6000 E-04$

0.7432

0.1520

$6.3279 E-08$

1.456

0.1268

0.1602

0.1506

0.2794

0.1506

0.2099

5.6000E-04

0.7432

0.1520

$6.3279 E-08$

0.0014

0.1268

0.1602

0.2479

0.4248

1.456

0.0014

0.2479

0.3450

5. $6000 E-04$

0.1064

0.7532

0.2679

$.0634 E-08$

0.1535

0.2595

0.4248

1.829

0.0027

0.2595

0.7532

0.3450

5.6OOOE- 04

0.1064

0.2679

$.0634 E-08$

0.1535

0.3753

0.4857

1.829

0.0027

0.3753

0.4078

5.6000E-04

0.0963

0.7589

0.3274

$9407 E-08$

0.1504

0.3940

0.4857

2. 079

0.0035

0.3940

0.4078

5.6000E-04

0.7589

0.3274

5. $9407 E-08$

2.079

0.0963

0.1504

0.0035

(CONT INUED)。

REI
REO
RELM

3138, 1.4786E-02

2363.

2642 。

2536。1.1559E-02

2237 。

2354 。

2536.1.1559E-02

2237 。

2354 。

2536 。

2237 。

2354 。

5279.

2833 .

3633 。

52790

2833 .

3633 .

5279.

2833 。

3633 。

4382 .

2644 。

3231 .

4382 .

2644 。

3231 。

3791 。

2522 。

2964 .

$3138 \circ 1.4786 E-02$

2363 。

2642 。

3138。1.4786E-02

2363.

2642 。 
AR NH3 , 0.790 MICRON D. PARTICLES (CONTIVUED)。

\begin{tabular}{|c|c|c|c|c|c|c|c|}
\hline $\begin{array}{l}\text { RUN } \\
\text { TYPE }\end{array}$ & $\begin{array}{l}\text { YINTI } \\
\text { YINTO } \\
\text { YINTLM }\end{array}$ & $\begin{array}{l}\text { EPEXP } \\
\text { EPCORR } \\
\text { EMT }\end{array}$ & $\begin{array}{l}\text { EPMO } \\
\text { EPSW } \\
\text { EPMA }\end{array}$ & $\begin{array}{l}\text { QINT } \\
\text { PLM } \\
\text { ULM }\end{array}$ & $\begin{array}{l}\text { FILMR } \\
\text { KN } \\
\text { UR }\end{array}$ & $\begin{array}{l}\text { REI } \\
\text { RED } \\
\text { RELM }\end{array}$ & MTC \\
\hline 920 & $\begin{array}{l}0.6240 \\
0.8660 \\
0.7588\end{array}$ & $\begin{array}{l}0.1462 \\
0.1462 \\
0.7432\end{array}$ & $\begin{array}{l}0.2794 \\
0.2099 \\
0.1520\end{array}$ & $\begin{array}{l}5.6000 E-04 \\
6.3279 E-08 \\
1.456\end{array}$ & $\begin{array}{l}0.1268 \\
0.1602 \\
0.0014\end{array}$ & $\begin{array}{l}31380 \\
23630 \\
26420\end{array}$ & $1.4786 E$ \\
\hline
\end{tabular}


TABLE XXI

DATA FOR CF2CL2, NH3

- 0.790 MICRON DIAMETER PARTICLES。

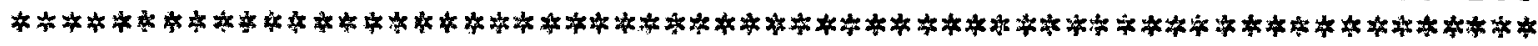

THREE CONSTANT INERT GAS FLOW RATES USED.

INLET TEMP。 (DEG。K) 293.0 , PRESSURE (ATM。) 1.000

CALIBRATION CORRECTION: COUNT/TRUE COUNT

INLET: $1.0000+0.0$ YINTI + 0.0308*YTRNI/YINTI

OUTLET: $1.0000+0.0$ *YINTO + 0.0308 *YRNO/YINTO

GAS

CF 2CL 2

(INERT)

MOLEC O WEIGHT
DENSITY
VISCOSITY
MEAN FREE PATH
VOLUME $\$(1 / 3)$

DIFFUS IVITY

(G/GMOLE)

(KG/M**3)

(KG/M/SEC)

(M)

(M/GMOLE* $*(1 / 3))$

(M*2/SEC)
$\mathrm{NH} 3$

(TRANSFERRED)

$\begin{array}{ll}120.9 & 17.03 \\ 5.136 & 0.7182 \\ 1.2500 E-05 & 9.8200 E-06 \\ 2.1800 E-08 & 4.5700 E-08 \\ 4.3310 E-02 & 2.2240 E-02\end{array}$

1. 2991E-05

\begin{tabular}{|c|c|c|c|c|c|c|c|}
\hline $\begin{array}{l}\text { UN } \\
\text { YPE }\end{array}$ & $\begin{array}{l}\text { YINTI } \\
\text { YINTO } \\
\text { YINTLM }\end{array}$ & $\begin{array}{l}\text { EPEXP } \\
\text { EPCDRR } \\
\text { EMT }\end{array}$ & $\begin{array}{l}\text { EPMO } \\
\text { EPSW } \\
\text { EPMA }\end{array}$ & $\begin{array}{l}\text { QINT } \\
\text { PLM } \\
\text { ULM }\end{array}$ & $\begin{array}{l}\text { FILMR } \\
\text { KN } \\
\text { UR }\end{array}$ & $\begin{array}{l}\text { REI } \\
\text { RED } \\
\text { KELM }\end{array}$ & MTC \\
\hline $\begin{array}{l}2807 \\
1\end{array}$ & $\begin{array}{l}0.1400 \\
0.8300\end{array}$ & $\begin{array}{l}6455 \\
5811\end{array}$ & $\begin{array}{l}8313 \\
6742\end{array}$ & $\begin{array}{l}908000 E-05 \\
1.9825 E-08\end{array}$ & $\begin{array}{l}0.0816 \\
0.0502\end{array}$ & & 1 \\
\hline & & & 4484 & & 0.0 & & \\
\hline 2807 & & & & & & & $1.2539 E-0$ \\
\hline & & & & & 0 . & 8. & \\
\hline & $0 .:$ & & 13 & 05 & 0 。 & 6́o & $1.2539 E-0$ \\
\hline & & & & & & & \\
\hline & & & & $E-05$ & 0.0 & & $1.2539 E-02$ \\
\hline & $\begin{array}{l}0.8 \\
0.4\end{array}$ & & & $\begin{array}{l}1 \\
0\end{array}$ & 0.0 & & \\
\hline 2813 & $\begin{array}{l}0.2 \\
0.9 \\
0.6\end{array}$ & & & & $\begin{array}{l}0.0 \\
0.0 \\
0.0\end{array}$ & & $1.0323 \mathrm{E}-$ \\
\hline & & & & & & & .0 \\
\hline & & & & 08 & & & \\
\hline 2816 & & & & $\begin{array}{l}05 \\
08\end{array}$ & & & $8.4315 E-$ \\
\hline & & & & & & & \\
\hline $32 \varepsilon$ & $\begin{array}{l}0.4 \\
0.9 \\
0.7\end{array}$ & & & $E-08$ & $\begin{array}{l}0 . \\
0 . \\
0 .\end{array}$ & $\begin{array}{l}9 . \\
2 . \\
1 .\end{array}$ & .4315 \\
\hline 28 & $\begin{array}{l}0.4300 \\
0.9700 \\
0.7828\end{array}$ & $\begin{array}{l}0.3341 \\
0.9767\end{array}$ & $\begin{array}{r}0.5567 \\
0.3244 \\
0.1536\end{array}$ & $\begin{array}{l}0 E-05 \\
9 E-08\end{array}$ & $\begin{array}{l}0.1 \\
0.0 \\
0.0\end{array}$ & $\begin{array}{l}2579 \circ \\
20322^{\circ}\end{array}$ & $8.4315 E-03$ \\
\hline
\end{tabular}




\section{CF 2 CL2, $\mathrm{NH}_{3}$}

RUN YINTI.

TYPE YINTO

YINTLM

EPEXP

,

0.790 MICRON D.

PARTICLES

EPCORR EPSW

EMT EPMA

QINT
PLM
ULM

FILMR
KN

UR

$\begin{array}{ll}32816 & 0.4300 \\ 11 & 0.9700 \\ 32817 & 0.782 \\ 11 & 0.6510 \\ & 0.888 \\ 32817 & 0.6510 \\ 11 & 0.988 \\ & 0.8897 \\ 32817 & 0.6510 \\ 11 & 0.9880 \\ 32817 & 0.08697 \\ 11 & 0.9880 \\ & 0.8897 \\ 32817 & 0.6510\end{array}$

328170.6510

I1 0.9880

0.8897

$32818 \quad 0.1840$

I1 0.8900

0.5549

328180.1840

I 10.8900

0.5549

328180.1840

I 1 0.8900

0.5549

328220.2850

I $3 \quad 0.7740$

0.5333

328220.2850

130.7740

0.5333

$32822 * 0.2850$

$13 \quad 0.7740$

0.5333

328220.2850

130.7740

0.5333

328220.2850

I $3 \quad 0.7740$

0.5333

328260.5970

I 2

0.9550

0.8233

328260.5970

I 2

0.9550

0.8233
$0.3640 \quad 0.5567908000 E-0.50 .1213$

$0.3387 \quad 0.3244 \quad 1.9239 E-08 \quad 0.0487$

0.9767

0.0977

0.0832

0.9773

0.1022

0.0877

0.9773

0.1181

0.1039

0.9773

0.1271

0.1130

0.9773

0.1454

0.1316

0.9773

0.5767

0.5208

0.9721

0. 5921

0.5382

0.9721

0.5843

0.5294

0.9721

0.3678

0.3251

0. 8836

0.3483

0.3042

0.8836

0.3520

0.3082

0.8836

0.3625

0.3194

0.8836

0.3429

0.2985

0.8836

0.0516

0.0333

0.9302

0.0614

$0.1536 \quad 0.2471$

0.0014

0.3411

0.1637

9. $8000 \mathrm{E}-05$

0.0686

2.0124E-08

0.2174

0.3411

9.8000E-05

$0.16372 .0124 E-08$

0.06860 .2174

0.3411

. 8174

0.1637

0.0686

2. $0124 E-08$

0.3411

0.1637

0.2174

$2.0124 E-08$

0.1430

$0.0686 \quad 0.2174$

0.3411

0.1637

9.8000E-05

0.0686

0.7933

0.2174

0.0509

0.6072

9. $8000 E-05$

0.3737

1. $9195 \mathrm{E}-08$

0.0486

0.7933

0.3486

0.6072

0.3737

0.7933

0.6072

0. 3737

0.6318

0.4285

0.2307

0.6318

0.4285

0.2307

$908000 E-05$

0.0876

$1.9195 E-08$

0.3486

9. $8000 E-05$

1.9195E-08

0.3486

2.4800E-04

$1.9318 E-08$

0.9177

2.4800E-04 0.0719

1.9318E-08 0.0489

0.9177

0.6318

2.4800E-04

0.4285

$1.9318 E-08$

$0.2307 \quad 0.9177$

0.6318

2.4800E-04

0.4285

1. $9318 E-08$

0.2307

0.9177

0.6318

$2.4800 E-04$

0.4285

$1.9318 E-08$

0.23070 .9177

0.0023

$0.37491 .9200 E-040.1014$

0.1880

1. $9502 E-08$

0.0808

0.4602

0.0007

0.0433

0.9302

0.3749

1. $9200 E-04$

0.1880

1. $9502 E-08$

0.0808
..8000E-05
0.1430

0.0509

0.0006

0.1430

0.0509

0.0006

0.1430

0.0509

0.0006

0.0509

0.0006

0.1430

0.0006

0.0876

0.0045

0.0486

0.0045

0.0876

0.0486

0.0045

0.0719

0.0489

0.0023

0.0023

0.0719

0.0489

0.0023

0.0719

0.0489

0.0023

0.0719

0.0489

0.0494

0.1014
(こONTINUED)。

REI

REO

RELM

2579. 8.4315E-03

2032 .

2141 .

2252 。

2024 .

2073 。

2252 。

20240

2073。

2252 。

2024 。

2073.

2252 .

2024 。

2073.

2252 。

2024 。

2073 。

3786 。

2073 .

2364 .

3786 。

2073.

2364 。

3786 。

2073.

2364 。

7714. $1.4232 E-02$

5433 。

6058 。

7714. 1.4232E-02

5433 。

6058 。

$7714^{\circ}$

5433 .

6058 。

7714.1.4232E-02

5433 。

6058 。

7714 。

5433 。

6058 .

4527 。

3996 。

4140 。

4527.1.0092E-02

3996.

4140 。

7. $1537 E-03$

7. $1537 E-03$

7. 1537E-03

$7.1537 E-03$

7. $1537 E-03$

$1.1673 E-02$

$1.1673 E-02$

$1.1673 E-02$

$1.4232 E-02$

$1.4232 E-02$

1. $0092 E-02$
0.0494

0.0007 
CF $2 \mathrm{CL} 2, \mathrm{NH} 3$

$\begin{array}{ll}\text { RUN } & \text { YINTI } \\ \text { TYPE } & \text { YINTO } \\ & \text { YINTLM } \\ 32826 & 0.5970 \\ 12 & 0.9550 \\ & 0.8233 \\ 32826 & 0.5970 \\ \text { I2 } & 0.9550 \\ & 0.8233\end{array}$

328310.5970

I 0.9550

0.8233

328310.5970

120.9550

0.8233

328310.5970

I 20.9550

0.8233

328310.5970

I2 0.9550

0.8233

328310.5970

120.9550

0.8233

328330.4120

I2 0.9020

0.6947

328330.4120

I 0.9020

0.6947

328330.4120

I2 0.9020

0.6947

328330.4120

I 20.9020

0.6947

328350.2450

13: 0.7130

0.4725

328350.2450

I 30.7130

0.4725

328350.2450

I $3 \quad 0.7130$

0.4725

328350.2450

130.7130

0.4725

328350.2450

13

0.7130

0.4725
,

EPEXP EPMO

EPCORR EPSW

EMT

EPMA

$0.06490 .3749 \quad 1.9200 E-04 \quad 0.1014$

0.0469

0.1880

(

1. $9502 E-08$

0.0494

0.9302

0.0808

0.4602

0.0007

0.0544

0. 3749

1. $9200 E-04$

0.1014

0.0362

0.1880

$1.9502 E-08$

0.0494

0. 9302

0.0808

0.4602

0.0007

0.1103

0.3749

$1.9200 E=04$

0.1014

0.0931

0.1880

$1.9502 E-08$

0.0494

0.9302

0.0808

0.4602

0.0007

0.1342

0.3749

1.9200E-04

0.1014

0.1175

0.1880

1. $9502 \mathrm{E}-08$

0.0494

0. 9302

0.0808

0.4602

0.0007

0.1076

0.3749

1.9200E-04

0.1014

0.0904

0.1880

$1.9502 E-08$

0.0494

0.9302

0.0808

0.1173

0.3749

0.4602

0.0007

0.1003

0. 1880

$1.9200 E-04$

0.1014

0.9302

0.0808

1. $9502 E-08$

0.0494

0.1386

0.3749

0.4602

0.0007

0. 1220

0.1880

1.9200E-04

0.1014

0.9302

0.0808

1. $9502 E-08$

0. 0494

0.1290

0.5432

0.4602

0.0007

0.0938

0.3222

1.9200E-04

0.0904

0.9239

0.1546

0.1293

0.5432

1. $8940 E-08$

0.0480

0.5454

0.0014

0.09410 .3222

1.9200E-04

0.0904

0.9239

0.1546

1. $8940 E-08$

0.0480

0.1492

0. 5432

0.5454

0.0014

0.11480 .3222

0.9239

0.1546

1.9200E-04

0.0904

1.8940E-0 8

0.5454

0.0480

0.17740 .5432

1.9200E-04

0.0014

0.14410 .3222

1.8940E-08

0.0904

0.9239

0.1546

0.5454

0.0480

0.1708

0.6564

2. 480OE-04

0.0014

0.1033

0.4662

$1.9799 E-08$

1.036

0.0709

0.8694

0.2631

2.4800E-04

0.0501

0.0027

0.18020 .6564

2.4800E-04

0.1134

0.4662

1.036

0.0709

0. 8694

0.2631

0.0501

$0.1827 \quad 0.6564$

2. 4800E-04

0.1161

0.4662

$1.9799 E-08$

0.0027

0.8694

0.2631

1. 036

0.0709

0.2222

0.1589

0.6564

2.4800E-04

0.0501

0.0027

0.8694

0.4662

1. $9799 E-08$

0.0709

0.2407

0.2631

1.036

0.0501

0.0027

0.1789

0.6564

$2.4800 E-04$

0.869 .4

0.2631

1. $9799 E-08$

0.0709

0.0501

0.0027

(CDNTINUED)。

REI
REO
RELM

MTC

4527.1.0092E-02

3996 。

4140 。

4527.1.0092E-02

3996.

4140 。

4527.

3996 .

4140.

4527.1.0092E-02

3996 .

4140 。

4527.1.0092E-02

3996.

4140 。

4527. 1.0092E-02

3996 .

4140 .

4527. 1.0092E-02

3996 。

41400

5133. 1.1313E-02

4049.

4331.

5133. 1.1313E-02

4049.

4331 。

$5133.1 .1313 E-02$

4049。

4331 。

5133。1.1313E-02

4049 。

+331 。

8276 。

5554 。

6310 。

8276

5554 。

6310 。

8276 .

5554 。

6310 。

8276 .

5554 。

6310 。

8276 .

5554 。

1. $4425 E-02$

$1.4425 E-02$

1. $4425 E-02$

1. $4425 E-02$

$1.4425 \varepsilon-02$

6310 。 


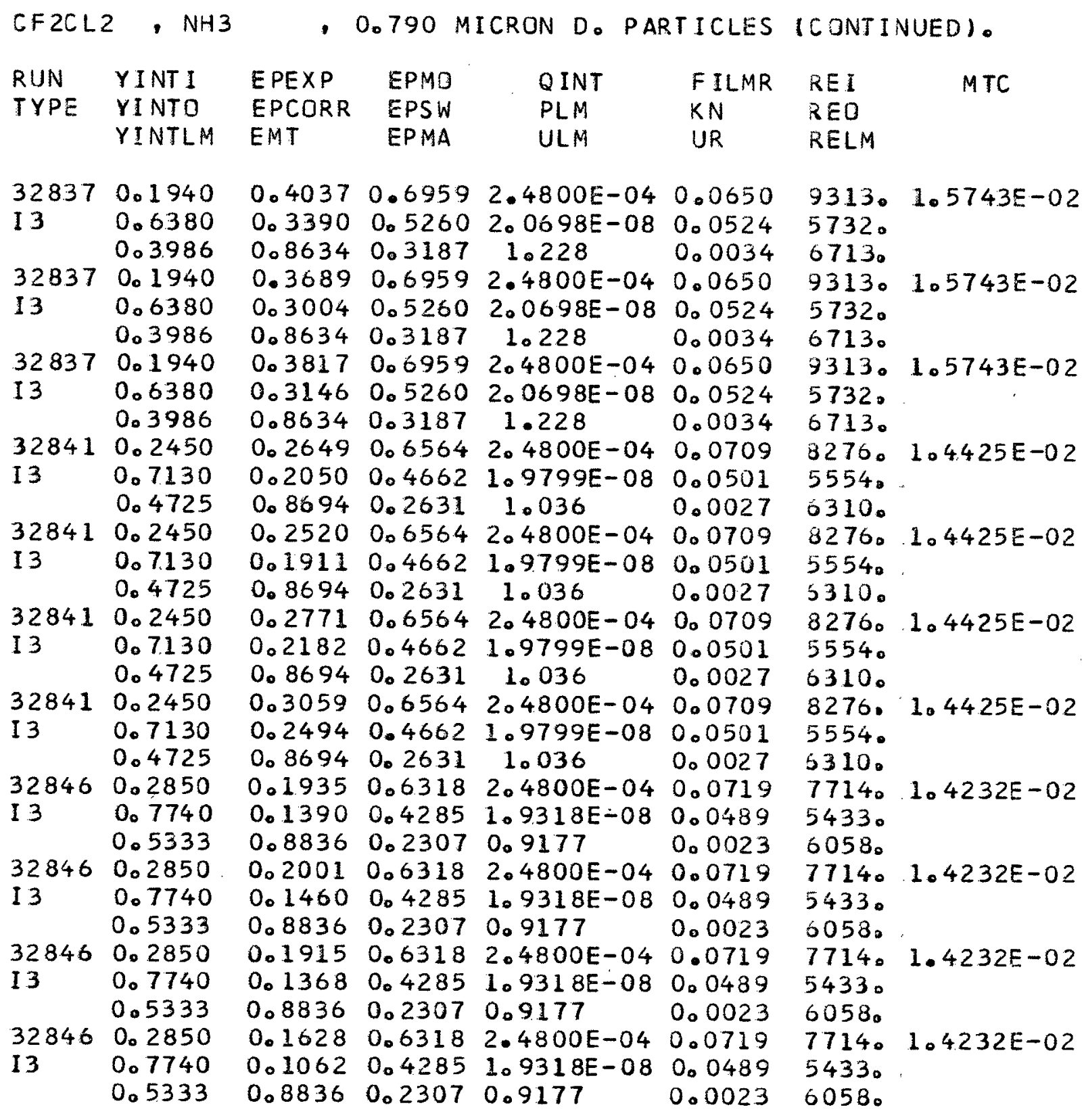


TABLE XXII

DATA FOR CF2CL2, NH3

, 1.011 MIERON DIAMETER PARTICLES。

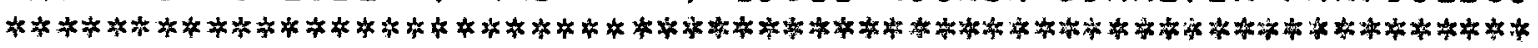

INERT GAS FLOW FLOW RATE CONSTANT IN ALL RUNS。

INLET TEMP。 (DEG。K) 293.0 , PRESSURE (ATM。) 1.000

CALI BRATION CORRECTION: COUNT/TRUE COUNT

INLET: $1.0000+0.0$ *YNTI + 0.0 *YTRNI/YINTI

OUTLET: $100000+0.0$ *YINTO + 0.0 \#YTRNO/YIVTJ

GAS

CF $2 C L 2$

(INERT)

$\begin{array}{ll}\text { MOLEC WEIGHT } & (G / G M O L E) \\ \text { DENSITY } & (K G / M * 3) \\ \text { VISCOSITY } & (K G / M / S E C) \\ \text { MEAN FREE PATH } & (M) \\ \text { VOLUME* }(1 / 3) & (M / G M O L E * *(1 / 3))\end{array}$

DIFFUSIVITY

$(M * 2 / S E C)$

$\begin{array}{ll}120.9 & 17.03 \\ 5.136 & 0.7182 \\ 1.2500 E-05 & 9.8200 E-06 \\ 2.1800 E-08 & 4.5700 E-08 \\ 4.3310 E-02 & 2.2240 E-02\end{array}$

$1.2991 E-05$

\begin{tabular}{|c|c|c|c|c|c|c|c|}
\hline $\begin{array}{l}\text { RUN } \\
\text { TYPE }\end{array}$ & $\begin{array}{l}\text { YINTI } \\
\text { YINTO } \\
\text { YINTLM }\end{array}$ & $\begin{array}{l}\text { EPEXP } \\
\text { EPCORR } \\
\text { EMT }\end{array}$ & $\begin{array}{l}\text { EPMD } \\
\text { EPSW } \\
\text { EPMA }\end{array}$ & $\begin{array}{l}\text { QINT } \\
\text { PLM } \\
\text { ULM }\end{array}$ & $\begin{array}{l}\text { FILMR } \\
\text { KN } \\
\text { UR }\end{array}$ & $\begin{array}{l}\text { REI } \\
\text { REO } \\
\text { RELM }\end{array}$ & MTC \\
\hline 36475 & $\begin{array}{l}0.1400 \\
0.8300 \\
0.4699\end{array}$ & $\begin{array}{l}0.6686 \\
0.6686 \\
0.9667\end{array}$ & $\begin{array}{l}0.8313 \\
0.6742 \\
0.4484 \\
0.8213\end{array}$ & $\begin{array}{l}9.8000 E-05 \\
1.9825 E-08 \\
0.4116\end{array}$ & $\begin{array}{l}0.0816 \\
0.0392 \\
0.0060\end{array}$ & $\begin{array}{l}4426 \circ \\
2109 \circ \\
2498^{\circ}\end{array}$ & $102539 E-02$ \\
\hline 36475 & $\begin{array}{l}0.1400 \\
0.8300 \\
0.4699\end{array}$ & $\begin{array}{l}0.6604 \\
0.6604 \\
0.9667\end{array}$ & $\begin{array}{l}0.8313 \\
0.6742 \\
0.4484\end{array}$ & $\begin{array}{l}9.8000 E-05 \\
1.9825 E-08 \\
0.4116\end{array}$ & $\begin{array}{l}0.0816 \\
0.0392 \\
0.0060\end{array}$ & $\begin{array}{l}4426 \circ \\
2109 \circ \\
2498^{\circ}\end{array}$ & $1.2539 E-02$ \\
\hline 36475 & $\begin{array}{l}0.1400 \\
0.8300 \\
0.4699\end{array}$ & $\begin{array}{l}0.6575 \\
0.6575 \\
0.9667\end{array}$ & $\begin{array}{l}0.8313 \\
0.6742 \\
0.4484\end{array}$ & $\begin{array}{l}9.8000 E-05 \\
1.9825 E-08 \\
0.4116\end{array}$ & $\begin{array}{l}0.0816 \\
0.0392 \\
0.0060\end{array}$ & $\begin{array}{l}4420 \\
2109 . \\
2498 .\end{array}$ & $.2539 E-02$ \\
\hline 6476 & $\begin{array}{l}0.1840 \\
0.8900 \\
0.5549 \\
0.1840\end{array}$ & $\begin{array}{l}0.6132 \\
0.6132 \\
0.9721 \\
0.6049\end{array}$ & $\begin{array}{l}0.7933 \\
0.6072 \\
0.3737 \\
0.7933\end{array}$ & $\begin{array}{l}9.8000 E-05 \\
1.9195 E-08 \\
0.3486 \\
9.8000 E-05\end{array}$ & $\begin{array}{l}0.0876 \\
0.0380 \\
0.0045 \\
0.0876\end{array}$ & $\begin{array}{l}3786^{\circ} \\
2073 . \\
2364^{\circ} \\
3786 .\end{array}$ & $73 E-02$ \\
\hline+76 & $\begin{array}{l}0.8900 \\
0.5549 \\
0.1840\end{array}$ & $\begin{array}{l}0.6049 \\
0.9721 \\
0.5937\end{array}$ & $\begin{array}{l}0.6072 \\
0.3737 \\
0.7933\end{array}$ & $\begin{array}{l}1.9195 E-08 \\
0.3486 \\
9.8000 E-05\end{array}$ & $\begin{array}{l}0.0380 \\
0.0045 \\
0.0876\end{array}$ & $\begin{array}{l}2073 \circ \\
2364^{\circ} \\
3786 。\end{array}$ & $.16 / 3 E-02$ \\
\hline & $\begin{array}{l}0.8900 \\
0.5549\end{array}$ & $\begin{array}{l}0.5937 \\
0.9721\end{array}$ & $\begin{array}{l}0.6072 \\
0.3737\end{array}$ & $\begin{array}{l}1.9195 E-08 \\
0.3486\end{array}$ & $\begin{array}{l}0.0380 \\
0.0045\end{array}$ & & \\
\hline 6477 & $\begin{array}{l}0.2600 \\
0.9340 \\
0.6513\end{array}$ & $\begin{array}{l}0.4930 \\
0.4930 \\
0.9752\end{array}$ & $\begin{array}{l}0.7216 \\
0.5036 \\
0.2790\end{array}$ & $\begin{array}{l}9.8000 E-05 \\
1.8923 E-08 \\
0.2970\end{array}$ & $\begin{array}{l}0.0991 \\
0.0374 \\
0.0030\end{array}$ & $\begin{array}{l}3179^{\circ} \\
2050^{\circ} \\
2251^{\circ}\end{array}$ & $1.0323 E-02$ \\
\hline 36477 & $\begin{array}{l}0.2600 \\
0.9340 \\
0.6513\end{array}$ & $\begin{array}{l}0.4781 \\
0.4781 \\
0.9752\end{array}$ & $\begin{array}{l}0.7216 \\
0.5036 \\
0.2790\end{array}$ & $\begin{array}{l}9.8000 E-05 \\
1.8923 E-08 \\
0.2970\end{array}$ & $\begin{array}{l}0.0991 \\
0.0374 \\
0.0030\end{array}$ & $\begin{array}{l}3179^{\circ} \\
2050^{\circ} \\
22510\end{array}$ & $1.0323 E-02$ \\
\hline 36477 & $\begin{array}{l}0.2600 \\
0.9340 \\
0.6513\end{array}$ & $\begin{array}{l}0.4663 \\
0.4663 \\
0.9752\end{array}$ & $\begin{array}{l}0.7216 \\
0.5036 \\
0.2790\end{array}$ & $\begin{array}{l}9.8000 E-05 \\
1.8923 E-08 \\
0.2970\end{array}$ & $\begin{array}{l}0.0991 \\
0.0374 \\
0.0030\end{array}$ & $\begin{array}{l}3179^{\circ} \\
2050^{\circ} \\
22510\end{array}$ & $1.0323 E-02$ \\
\hline
\end{tabular}

$\mathrm{NH} 3$

(TRANSFERRED)

$$
\begin{aligned}
& 17.03 \\
& 0.7182 \\
& 9.8200 E-06 \\
& 4.5700 E-08 \\
& 2.2240 E-02
\end{aligned}
$$


CF2CL2, NH3 , 1.011 MICRON D. PARTICLES (CONTINUED)。

\begin{tabular}{|c|c|c|c|c|c|c|c|}
\hline $\begin{array}{l}\text { RUN } \\
\text { TYPE }\end{array}$ & $\begin{array}{l}\text { YINTI } \\
\text { YINTO } \\
\text { YINTLM }\end{array}$ & $\begin{array}{l}\text { EPEXP } \\
\text { EPCORR } \\
\text { EMT }\end{array}$ & $\begin{array}{l}\text { EPMO } \\
\text { EPS } \\
\text { EPMA }\end{array}$ & $\begin{array}{l}\text { OINT } \\
\text { PLM } \\
\text { ULM }\end{array}$ & $\begin{array}{l}\text { FILMR } \\
\text { KN } \\
\text { UR }\end{array}$ & $\begin{array}{l}\text { REI } \\
\text { REO } \\
\text { RELM }\end{array}$ & MTC \\
\hline 36474 & $\begin{array}{l}0.4300 \\
0.9700 \\
0.7828\end{array}$ & $\begin{array}{l}0.3098 \\
0.3098 \\
0.9767\end{array}$ & $\begin{array}{l}0.5567 \\
0.3244 \\
0.1536\end{array}$ & $\begin{array}{l}9.8000 E-05 \\
1.9239 E-08 \\
0.2471\end{array}$ & $\begin{array}{l}0.1213 \\
0.0381 \\
0.0014\end{array}$ & $\begin{array}{l}2579^{\circ} \\
2032{ }^{\circ} \\
2141 \%\end{array}$ & $8.4315 E-03$ \\
\hline 6474 & $\begin{array}{l}0.4300 \\
0.9700 \\
0.7828\end{array}$ & $\begin{array}{l}0.3077 \\
0.3077 \\
0.9767\end{array}$ & $\begin{array}{l}0.5567 \\
0.3244 \\
0.1536\end{array}$ & $\begin{array}{l}9.8000 E-05 \\
1.9239 E-08 \\
0.2471\end{array}$ & $\begin{array}{l}0.1213 \\
0.0381 \\
0.0014\end{array}$ & $\begin{array}{l}2579 \circ \\
2032^{\circ} \\
21410\end{array}$ & $8.4315 E-03$ \\
\hline 36474 & $\begin{array}{l}0.4300 \\
0.9700 \\
0.7828\end{array}$ & $\begin{array}{l}0.3173 \\
0.3173 \\
0.9767\end{array}$ & $\begin{array}{l}0.5567 \\
0.3244 \\
0.1536\end{array}$ & $\begin{array}{l}9.8000 E-05 \\
1.9239 E-08 \\
0.2471\end{array}$ & $\begin{array}{l}0.1213 \\
0.0381 \\
0.0014\end{array}$ & $\begin{array}{l}2579 \\
2032^{\circ} \\
2141^{\circ}\end{array}$ & $8.4315 E-03$ \\
\hline 36473 & $\begin{array}{l}0.6510 \\
0.9880 \\
0.8897\end{array}$ & $\begin{array}{l}0.1584 \\
0.1584 \\
0.9773\end{array}$ & $\begin{array}{l}0.3411 \\
0.1637 \\
0.0686\end{array}$ & $\begin{array}{l}9.8000 E-05 \\
2.0124 E-08 \\
0.2174\end{array}$ & $\begin{array}{l}0.14 \\
0.03 \\
0.00\end{array}$ & $\begin{array}{l}2252^{\circ} \\
2024^{\circ} \\
2073 \circ\end{array}$ & 7.15 \\
\hline 647 & $\begin{array}{l}0.6510 \\
0.9880 \\
0.8897\end{array}$ & $\begin{array}{l}0.1766 \\
0.1766 \\
0.9773\end{array}$ & $\begin{array}{l}0.3411 \\
0.1637 \\
0.0686\end{array}$ & $\begin{array}{l}9.8000 E-05 \\
2.0124 E-08 \\
0.2174\end{array}$ & $\begin{array}{l}0.14 \\
0.03 \\
0.00\end{array}$ & $\begin{array}{l}2252 . \\
2024 \circ \\
2073 .\end{array}$ & $7.1537 E-03$ \\
\hline 6 & $\begin{array}{l}0.6510 \\
0.9880 \\
0.8897\end{array}$ & $\begin{array}{l}0.1966 \\
0.1966 \\
0.9 .773\end{array}$ & $\begin{array}{l}0.3411 \\
0.1637 \\
0.0686\end{array}$ & $\begin{array}{l}9.8000 E-05 \\
2.0124 E-08 \\
0.2174\end{array}$ & $\begin{array}{l}0.1430 \\
0.0398 \\
0.0006\end{array}$ & $\begin{array}{l}2252 . \\
2024 \circ \\
20730\end{array}$ & $7.1537 \mathrm{E}-03$ \\
\hline
\end{tabular}


TABLE XXIII

DATA FOR CF2CL2, NH3

, 2.020 MICRON DIAMETER PARTICLES。

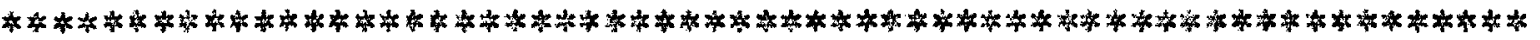

INERT GAS FLOW RATE CDNSTANT IN ALL RUNS.

INLET TEMP。 (DEG。 K) 293.0 , PRESSURE (ATM。) 1.000

CALIBRATION CORRECTION: COUNT/TRUE CDUNT

INLET: $\quad 100000+0.0$ \#YINTI + 0.0 *YTRNI/YINTI

DUTLET: $1.1400+-0.1400 * Y I N T O+0.0$ *YTRNO/YINTJ

GAS

$\begin{array}{ll}\text { MOLEC。 WEIGHT } & \text { (G/GMOLE) } \\ \text { DENSITY } & \text { (KG/M**3) } \\ \text { VISCOSITY } & (K G / M / S E C) \\ \text { MEAN FREE PATH } & \text { (M) } \\ \text { VOLUME }(1 / 3) & \text { (M/GMOLE* }(1 / 3))\end{array}$

DIFFUSIVITY (M\$2/SEC)

\section{CF 2 CL2 (INERT)}

$\begin{array}{ll}120.9 & 17.03 \\ 5.136 & 0.7182 \\ 1.2500 E-05 & 9.8200 E-06 \\ 2.1800 E-08 & 4.5700 E-08 \\ 4.3310 E-02 & 2.2240 E-02\end{array}$

$\mathrm{NH3}$

(TRANSFERRED)

$$
\begin{aligned}
& 17.03 \\
& 0.7182 \\
& 9.8200 E-06 \\
& 4.5700 E-08 \\
& 2.2240 E-02
\end{aligned}
$$

$\begin{array}{llllllll}\text { RUN } & \text { YINTI } & \text { EPEXP } & \text { EPMO } & \text { QINT } & \text { FILMR } & \text { REI } & \text { MTC } \\ \text { TYPE } & \text { YINTO } & \text { EPCORR } & \text { EPSW } & \text { PLM } & \text { KN } & \text { REO } & \\ & \text { YINTLM } & \text { EMT } & \text { EPMA } & \text { ULM } & \text { UR } & \text { RELM }\end{array}$

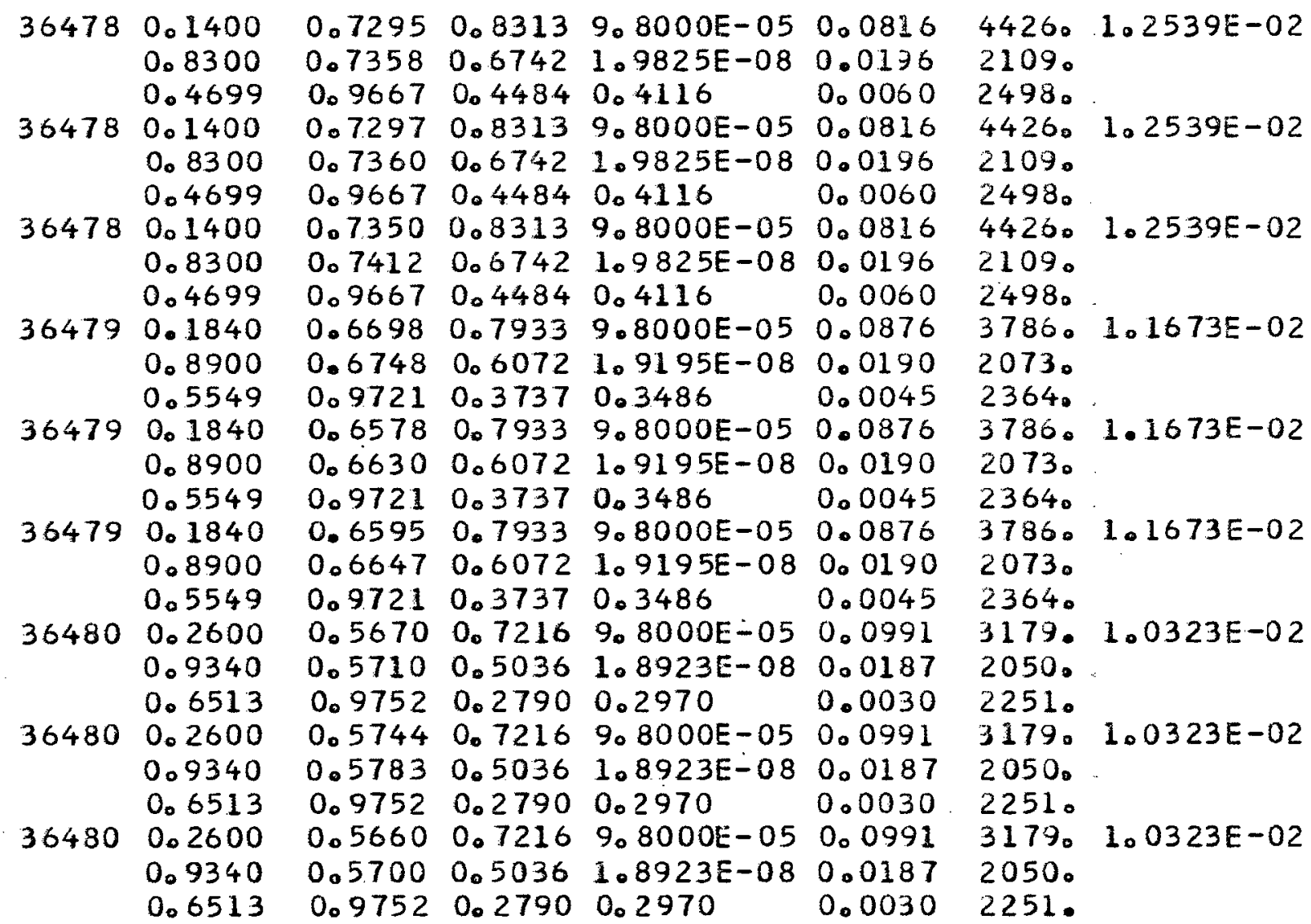

1.2991E-05 
CF2CL2, NH3 , 2.020 MICRON D。 PARTICLES (CONTINUED)。

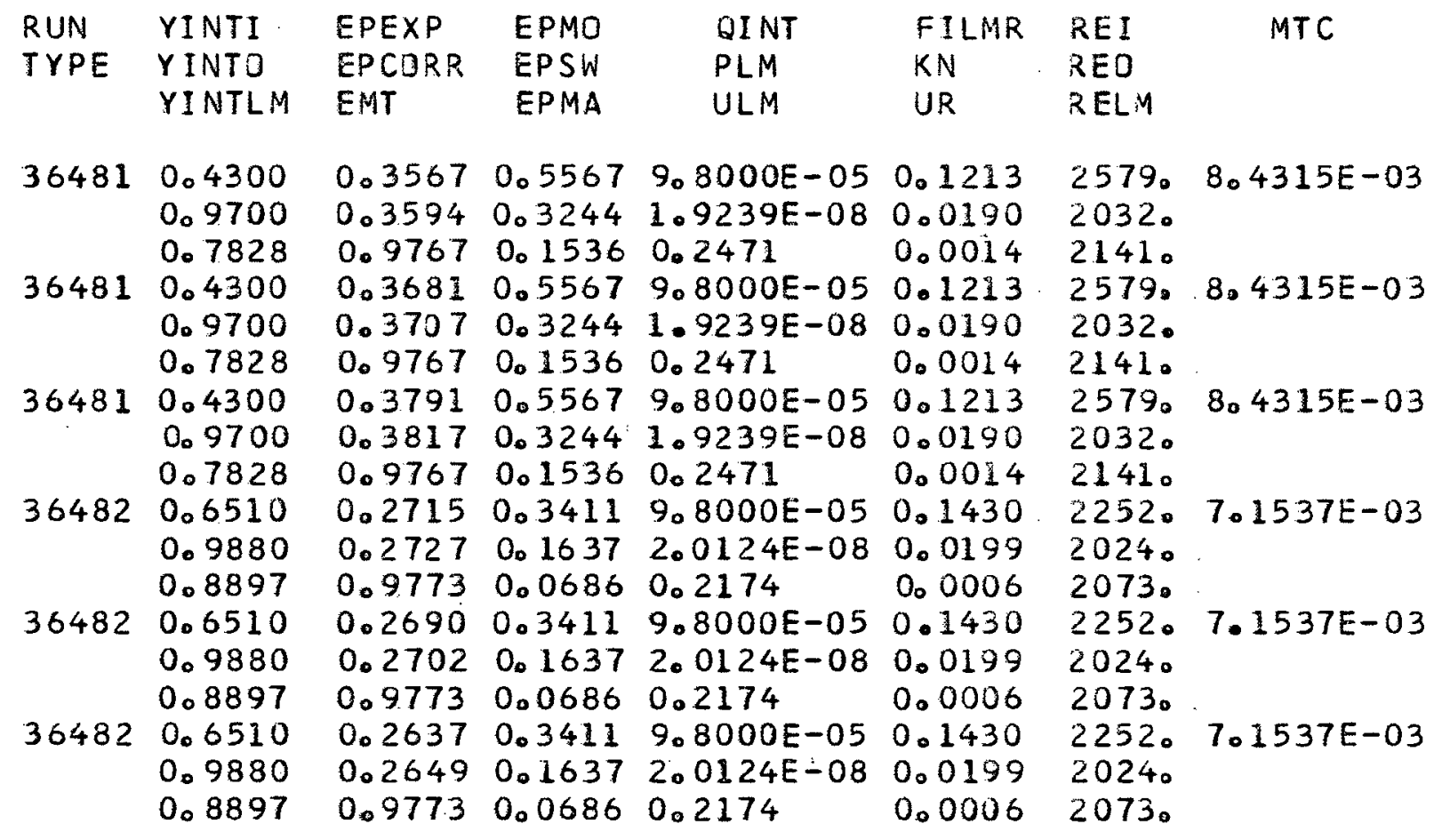




\section{TABLE XXIV}

DATA FOR N2 - N(CH3)3,0.500 MICRON DIAMETER PARTICLES。

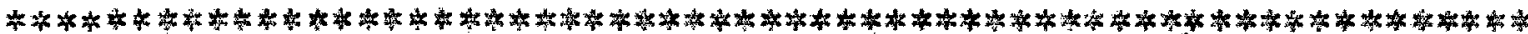

ALL RUNS FOR CONSTANT INERT RATE. INLET TEMP。 (DEG。K) 293.0 , PRESSURE (ATM。) 1.000

CALIBRATION CORRECTION: COUNT/TRUE COUNT INLET: $\quad 1.0000+0.0$ \#YINTI + 0.0 *YTRINI/YINTI OUTLET: $0.5600+0.4400$ \%INTO + 0.0 \#YTRNO/YINTJ

GAS

$\begin{array}{ll}\text { MOLEC。 WEIGHT } & \text { (G/GMOLE) } \\ \text { DENSITY } & (K G / M * 3) \\ \text { VISCOSITY } & \text { (KG/M/SEC) } \\ \text { MEAN FREE PATH } & (M) \\ \text { VOLUME* }(1 / 3) & (M / G M O L E * \%(1 / 3)) \\ \text { DIFFUSIVITY } & (M * 2 / S E C)\end{array}$

TYPE YINTO EPCORR EPSW YINTLM EMT EPMA

367620.6890

0.8340 0.7667

367620.6890 0.8340 0.7667

367620.6890 0. 8340 0.7667

367630.7780 0. 8870 0.8374

367630.7780 0.8870 0.8374

367630.7780 0.8870 0.8374

$36764 \quad 0.8300$ 0.9150 0. 8767

36764.0 .8300 0.9 .150 0.8767

367640.8300 0.9150 0.8767
N2 (INERT)

$$
\begin{array}{ll}
28.02 & 59.11 \\
1.165 & 2.510 \\
1.7480 E-05 & 7.5300 E-06 \\
6.3900 E-08 & 1.8800 E-08 \\
3.1190 E-02 & 4.5360 E-02
\end{array}
$$

N $1 \mathrm{CH} 313$ (TRANSFERRED)

$$
\text { 8. } 4411 E-06
$$



, N(CH3)3, 0.500 MICRON D. PARTICLES (CONTINUED).

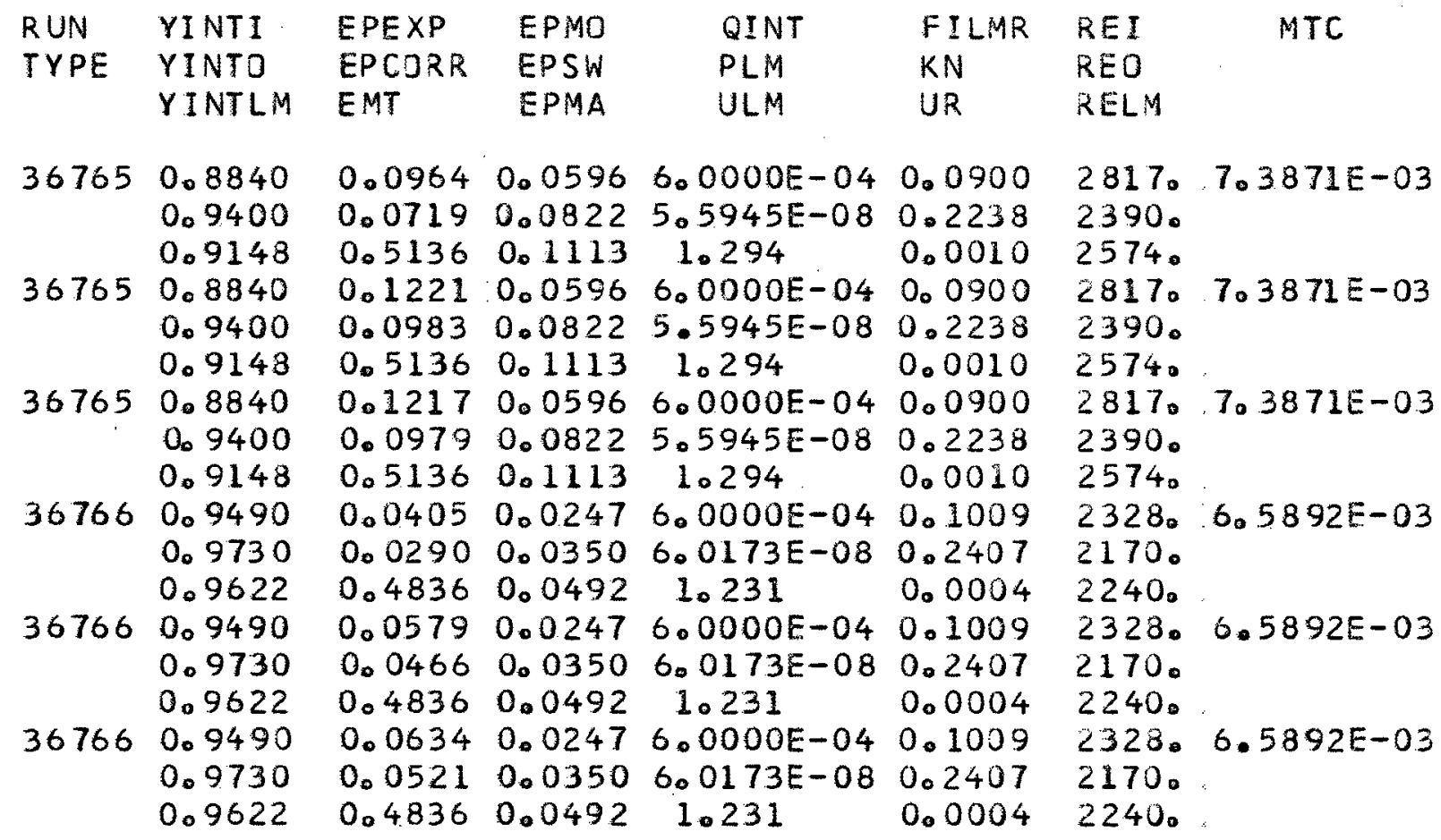




\section{TABLE XXV}

DATA FOR N2

- N(CH3)3, 0.790 MICRON DIAMETER PARTICLES。

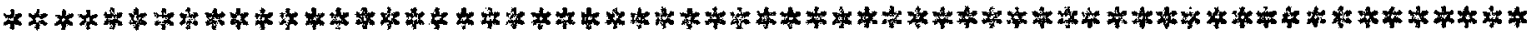

INERT GAS FLOW RATE CONSTANT IN ALL RUNS .

INLET TEMP。 (DEG。 K) 293.0 , PRESSURE (ATM。) 1.000

CALIBRATION CDRRECTION: COUNT/TRUE COUNT

INLET: $\quad 100000+0.0$ \#INTI + 0.0 \$YTRNI/YINTI

OUTLET: $0.6000+0.4000 \%$ YNTO + 0.0 *YTRNO/YINTJ

GAS

$\begin{array}{ll}\text { MOLEC . WEIGHT } & (\mathrm{G} / \mathrm{GMOLE}) \\ \text { DENSITY } & (K G / M * 3) \\ \text { VISCOSITY } & (K G / M / S E C) \\ \text { MEAN FREE PATH } & (M) \\ \text { VOLUME* }(1 / 3) & (M / G M O L E *(1 / 3)) \\ \text { DIFFUSIVITY } & (M * 2 / S E C)\end{array}$

$\begin{array}{llll}\text { RUN } & \text { YINTI } & \text { EPEXP } & \text { EPMO } \\ \text { TYPE } & \text { YINTO } & \text { EPCORR } & \text { EPSW } \\ & Y I N T L M & \text { EMT } & \text { EPMA }\end{array}$

367860.6890

0.8340

0.7667

0.2442

0.1904

0.5590

367860.6890

0.8340

0.7667

0.2799

0.2287

0.5590

0.2486

367860.6890

0. 8340

0.7667

$36786 \quad 0.6890$

0.8340

0.7667

367860.6890

0.8340

0.7667

36788 0.7780

0.8870

0.8374

$36788 \quad 0.7780$

0.8870

0.8374

$36788 \quad 0.7780$

0.8870

0.8374

367890.8300

0.9 .150

0.8767
0.1952

0.5590

0.2349

0.1805

0.5590

0.2339

0.1794

0.5590

0.2019

0.1641

0.5535

0.2094

0.1720

0.5535

0.1870

0.1485

0.5535

0.2110

0.1832

0.5465
N2

( INERT)

$$
\begin{aligned}
& 28.02 \\
& 1.165
\end{aligned}
$$

1. $7480 E-05$

6. $3900 E-08$

3. $1190 E-02$
$N(\mathrm{CH} 3) 3$

(TRANSFERRED)

\subsection{1 \\ 2.510}

7.5300E-06

1. $8800 E-08$

4. $5360 E-02$

\section{$8.4411 E-06$}

$\begin{array}{lll}\text { QINT } & \text { FILMR } & \text { REI } \\ \text { PLM } & \text { KN } & \text { REO } \\ \text { ULM } & \text { UR } & \text { RELM }\end{array}$

5053.9.2749E-03

3263 。

3988 。

5053.9.2749E-03

3263 。

3988 。

5053。.9.2749E-03

3263 。

3988 。

5053.9.2749E-03

3263 。

3988 .

$5053 \cdot 9.2749 E-03$

3263 。

$3988^{\circ}$

3855. 8.6912E-03

2792 。

3231 。

3855 . 8.6912E-03

2792 。

3231 。

3855 。

2792。

3231 .

3302 。

2572。

2878 。 
N2 - N(CH3)3, 0.790 MICRON Do PARTICLES (CONTINUED)。

\begin{tabular}{|c|c|c|c|c|c|c|c|}
\hline $\begin{array}{l}\text { RUN } \\
\text { TYPE }\end{array}$ & $\begin{array}{l}\text { YINTI } \\
\text { YINTO } \\
\text { YINTLM }\end{array}$ & $\begin{array}{l}\text { EPEXP } \\
\text { EPCORR } \\
\text { EMT }\end{array}$ & $\begin{array}{l}\text { EPMO } \\
\text { EPSW } \\
\text { EPMA }\end{array}$ & $\begin{array}{l}\text { QINT } \\
\text { PLM } \\
\text { ULM }\end{array}$ & $\begin{array}{l}\text { FILMR } \\
\text { KN } \\
\text { UR }\end{array}$ & $\begin{array}{l}\text { REI } \\
\text { REO } \\
\text { RELM }\end{array}$ & MTC \\
\hline 6789 & $\begin{array}{l}0.8300 \\
0.9150 \\
0.8767\end{array}$ & $\begin{array}{l}0.1658 \\
0.1364 \\
0.5465\end{array}$ & $\begin{array}{l}0.0929 \\
0.1253 \\
0.1649\end{array}$ & $\begin{array}{l}6.0000 E-04 \\
5.2869 E-08\end{array}$ & $\begin{array}{l}0.0800 \\
0.1338 \\
0.0015\end{array}$ & $\begin{array}{l}3302 . \\
2572 . \\
2878\end{array}$ & $8.3040 E-03$ \\
\hline 6789 & $\begin{array}{l}0.8300 \\
0.9150 \\
0.8767\end{array}$ & $\begin{array}{l}0.1176 \\
0.0865 \\
0.5465\end{array}$ & $\begin{array}{l}0.0929 \\
0.1253 \\
0.1649\end{array}$ & $\begin{array}{l}6.0000 E-04 \\
5.2869 E-08 \\
1.351\end{array}$ & $\begin{array}{l}0.0800 \\
0.1338 \\
0.0015\end{array}$ & $\begin{array}{l}3302^{\circ} \\
2572^{\circ} \\
2870^{\circ}\end{array}$ & $8.3040 E-03$ \\
\hline 6792 & $\begin{array}{l}0.8840 \\
0.9400 \\
0.9148\end{array}$ & $\begin{array}{l}0.0636 \\
0.0406 \\
0.5136\end{array}$ & $\begin{array}{l}0.0596 \\
0.0822 \\
0.1113\end{array}$ & $\begin{array}{l}6.0000 E-04 \\
5.5945 E-08 \\
1.294\end{array}$ & $\begin{array}{l}0.0900 \\
0.1416 \\
0.0010\end{array}$ & $\begin{array}{l}2817^{\circ} \\
2390 \% \\
25740\end{array}$ & 7. $3871 E-03$ \\
\hline 92 & $\begin{array}{l}0.8840 \\
0.9400 \\
0.9148\end{array}$ & $\begin{array}{l}0.1338 \\
0.112 ' 5 \\
0.5136\end{array}$ & $\begin{array}{l}0.0596 \\
0.0822 \\
0.1113\end{array}$ & $\begin{array}{l}6.0000 E-04 \\
5.5945 E-08 \\
1.294\end{array}$ & $\begin{array}{l}0.0900 \\
0.1416 \\
0.0010\end{array}$ & $\begin{array}{l}2817^{\circ} \\
2390^{\circ} \\
2574 \circ\end{array}$ & $3871 E-03$ \\
\hline 6792 & $\begin{array}{l}0.8840 \\
0.9400 \\
0.9148\end{array}$ & $\begin{array}{l}0.1543 \\
0.1335 \\
0.5136\end{array}$ & $\begin{array}{l}0.0596 \\
0.0822 \\
0.1113\end{array}$ & $\begin{array}{l}6.0000 E-04 \\
5.5945 E-08 \\
1.294\end{array}$ & $\begin{array}{l}0.0900 \\
0.1416 \\
0.0010\end{array}$ & $\begin{array}{l}2817 . \\
2390 。 \\
2574\end{array}$ & $7.3871 E-03$ \\
\hline 92 & $\begin{array}{l}0.8840 \\
0.9400 \\
0.9148\end{array}$ & $\begin{array}{l}0.1186 \\
0.0969 \\
0.5136\end{array}$ & $\begin{array}{l}0.0596 \\
0.0822 \\
0.1113\end{array}$ & $\begin{array}{l}6.0000 E-04 \\
5.5945 E-08 \\
1.294\end{array}$ & $\begin{array}{l}0.0900 \\
0.1416 \\
0.0010\end{array}$ & $\begin{array}{l}2817 \circ \\
2390 。 \\
2574 。\end{array}$ & $7.3871 \varepsilon-03$ \\
\hline 36793 & $\begin{array}{l}0.9490 \\
0.9730 \\
0.9622\end{array}$ & $\begin{array}{l}0.0529 \\
0.0426 \\
0.4836\end{array}$ & $\begin{array}{l}0.0247 \\
0.0350 \\
0.0492\end{array}$ & $\begin{array}{l}6.0000 E-04 \\
6.0173 E-08 \\
1.231\end{array}$ & $\begin{array}{l}0.1009 \\
0.1523 \\
0.0004\end{array}$ & $\begin{array}{l}2328 \circ \\
2170 \circ \\
2240 .\end{array}$ & 6.5 .8 \\
\hline 6793 & $\begin{array}{l}0.9490 \\
0.9730 \\
0.9622\end{array}$ & $\begin{array}{l}0.0415 \\
0.0310 \\
0.4836\end{array}$ & $\begin{array}{l}0.0247 \\
0.0350 \\
0.0492\end{array}$ & $\begin{array}{l}6.0000 E-04 \\
6.0173 E-08 \\
1.231\end{array}$ & $\begin{array}{l}0.1009 \\
0.1523 \\
0.0004\end{array}$ & $\begin{array}{l}2328^{\circ} \\
2170^{\circ} \\
2240^{\circ}\end{array}$ & $6.5892 E-03$ \\
\hline & $\begin{array}{l}0.9490 \\
0.9730 \\
0.9622\end{array}$ & $\begin{array}{l}0.0390 \\
0.0285 \\
0.4836\end{array}$ & $\begin{array}{l}0.0247 \\
0.0350 \\
0.0492\end{array}$ & $\begin{array}{l}\text { 6.0000E-04 } \\
6.0173 E-08 \\
1.231\end{array}$ & $\begin{array}{l}0.1009 \\
0.1523 \\
0.0004\end{array}$ & $\begin{array}{l}2328 . \\
2170 . \\
2240 .\end{array}$ & 6. \\
\hline
\end{tabular}


TABLE XXVI

DATA FOR N2 , N(CH3)3, 1.011 MICRON DIAMETER PARTICLES。

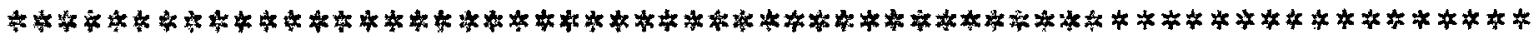

INERT GAS FLOW RATE CONSTANT IN ALL: RUNS 。

INLET TEMP。 (DEG。K) 293.0 , PRESSURE (ATM。) 1.000

CALIBRATION CORRECTION: COUNT/TRUE COUNT

INLET: $1.0000+0.0$ *YINTI + 0.0 \%YTRNI/YINTI

OUTLET: $1.0000+0.0$ *YNTO +.0.0 \#YTRNO/YIVTJ

GAS

$\begin{array}{ll}\text { MOLEC。 WEIGHT } & \text { (G/GMOLE) } \\ \text { DENSITY } & \begin{array}{l}(K G / M * * 3) \\ \text { VISCOSITY }\end{array} \\ \text { MEAN FREE PATH } & (M) \\ \text { VOLUME* } *(1 / 3) & (M / G M O L E * *(1 / 3)) \\ \text { DIFFUSIVITY } & (M * * 2 / S E C)\end{array}$

RUN YINTI

TYPE YINTO YINTLM

$\begin{array}{ll}\text { EPEXP } & \text { EPMO } \\ \text { EPCORR } & \text { EPSW } \\ \text { EMT } & \text { EPMA }\end{array}$

QINT

PLM

ULM
$\mathrm{N}(\mathrm{CH}) 3$

(TRANSFERRED)

$$
\text { (INERT) }
$$

$$
\begin{aligned}
& 28.02 \\
& 1.165
\end{aligned}
$$$$
\text { 1. } 7480 E-05
$$

6.3900E-08

$3.1190 E-02$
59.11

2.510

7.5300 E-06

1. $8800 E-08$

4. $5360 E-02$

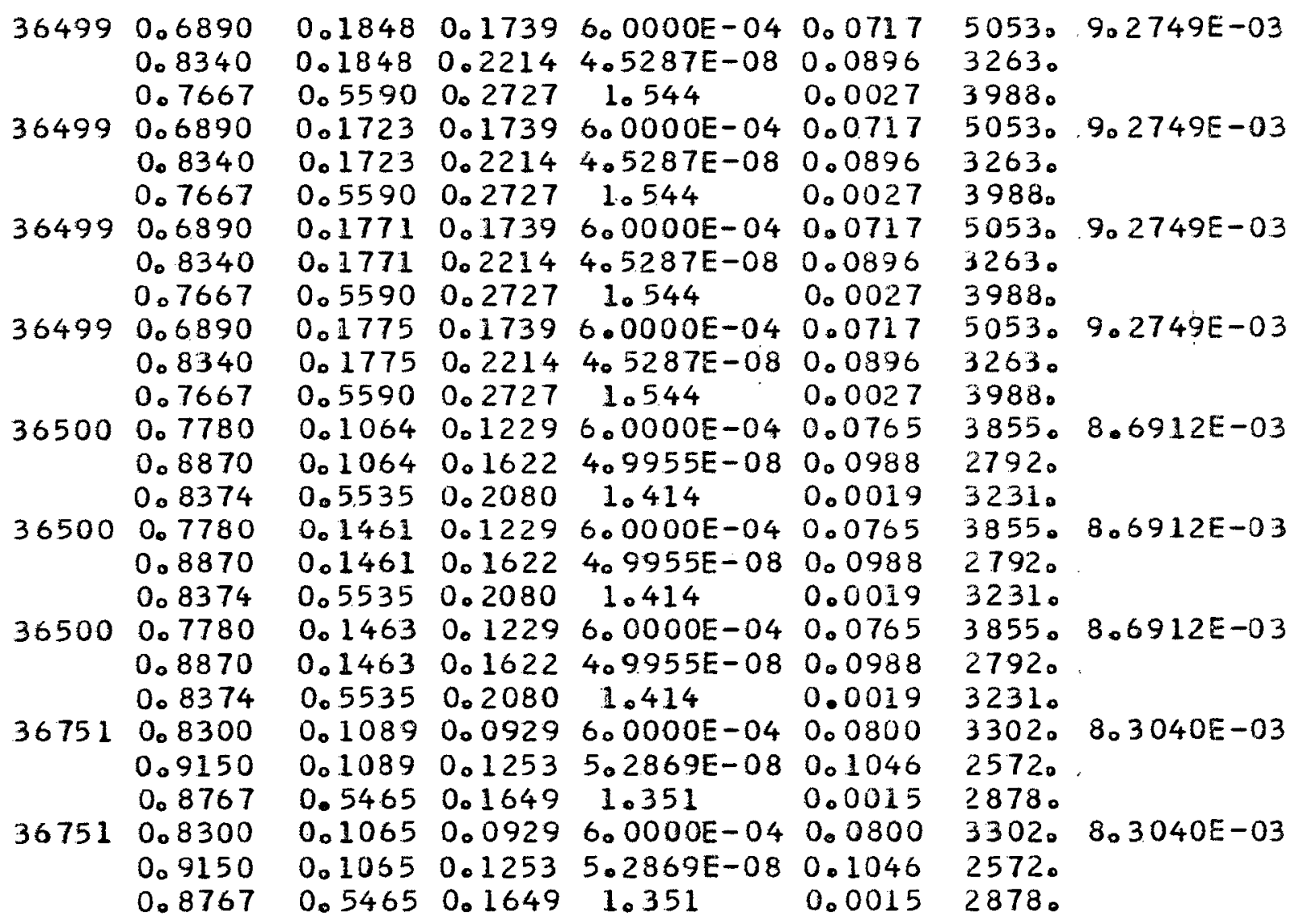


N2

$\begin{array}{ll}\text { RUN } & \text { YINTI } \\ \text { TYPE } & \text { YINTO } \\ & \text { YINTLM } \\ 36752 & 0.8840 \\ & 0.9400 \\ & 0.9148 \\ 36752 & 0.8840 \\ & 0.9400 \\ 36752 & 0.9148 \\ & 0.8840 \\ & 0.9400 \\ 36753 & 0.9148 \\ & 0.9490 \\ & 0.9730 \\ & 0.9622\end{array}$

367530.9490 0.9730 0.9622

$36753 \quad 0.9490$ 0.9730 0.9622

367530.9490 0.9730 0.9622

370620.6890 0.8340 0.7667

370620.6890 0.8340 0.7667

370620.6890 0.8340 0.7667

370630.7780 0. 8870 0.8374

$37063 \quad 0.7780$ 0. 8870 0.8374

$37063 \quad 0.7780$ 0.8870 0.8374

370640.8300 0.9150 0.8767

370640.8300 0.9150 0.8767

370640.8300 0.9150 0.8767

$\begin{array}{ll}\text { EPEXP } & \text { EPMO } \\ \text { EPCDRR } & \text { EPSW } \\ \text { EMT } & \text { EPMA }\end{array}$

QINT
PLM
ULM

FILMR
KN
UR

$0.0766 \quad 0.0596 \quad 6.0000 E-04 \quad 0.0900$ 0.0766

0.5136

0.1041

0.1041

0.5136

0.0927

0.0927

0.5136

0.0146

0.0146

0.4836

0.0160

0.0160

0.4836

0.0038

0.0038

0.4836

0.0192

0.0192

0.4836

0.1752

0.1752

0.5590

0.1463

0.1453

0.5590

0.1347

0.1347

0.5590

0.1424

0.1424

0.5535

0.1229

0.1229

0.5535

0.1409

0.1409

0.5535

0.1052

0. 1052

0.5465

0.0884

0.0884

0.5465

0.0982

0.0982

0. 5465

$$
0.08225 .5945 E-08
$$

$0.1113 \quad 1.294$

0.1107

0.0010

0.0596 6.0000E-04 0.0900

$0.08225 .5945 E-08$

$0.1113 \quad 1.294$

0.1107

0.0010

0.0596 6.0000E-04

$0.08225 .5945 E-08$

$0.1113 \quad 1.294$

0.0900

$0.02476 .00008-04$

$0.03506 .0173 E-08$

$0.0492 \quad 1.231$

0.0247 6.000OE-04

$0.0350 \quad 6.0173 E-08$

$0.0492 \quad 1.231$

$0.02476 .0000 E-04$

$0.0350 \quad 6.0173 E-08$

$0.0492 \quad 1.231$

0.0247 6.0000E-04

$0.03506 .0173 E-08$

0.04921 .231

0.1739 6. OOOOE-04

$0.2214 \quad 4.5287 \mathrm{E}-08$

$0.2727 \quad 1.544$

0.1107

0.0010

0.1009

0.1190

0.0004

0.1009

0.1190

0.0004

0.1009

0.1190

0.0004

0.1009

0.1190

0.0004

0.0717

0.0896

0.1739 6. OOOOE-04 0.0717

$0.22144 .5287 E-08$

0.0896

$0.2727 \quad 1.544$

0.0027

0.1739 6.0OOOE-04 0.0717

$0.2214 \quad 4.5287 E-08$

$0.2727 \quad 1.544$

0.0896

0.1229 6.0000E-04

0.1622 4.9955E-08

$0.2080 \quad 1.414$

0.0027

$0.12296 .0000 E-04$

0.1622 4.9955E-08

$0.2080 \quad 1.414$

$0.12296 .0000 E-04$

0.1622

4. $9955 E-08$

$0.2080 \quad 1.414$

$0.09296 .0000 E-04$

0.1253 5.2869E-08

$0.1649 \quad 1.351$

0.0765

0.0988

0.0019

0.0765

0.0988

0.0019

0.0765

0.0988

0.0019

0.0800

0.1046

0.0015

0.0929 6.0000E-04 0.0800

$0.1253502869 E-08$

$0.1649 \quad 1.351$

0.1046

0.0015

0.0929 6. OOOOE-04 0.0800

$0.12535 .2869 E-08$

0.1046

0.0015

(CONTINUEO).

REI

REO

RELM

2817。7.3871E-03

2390 。

2574 。

2817. 7.3871E-03

2390 。

2574 。

2817 .

2390 。

2574 。

2328 。

2170 。

2240 。

2328 。

2170 。

2240 。

23280

2170 。

2240 。

2328 。

2170 。

2240 。

5053.

3263 .

3988 。

5053 。

3263 。

3988 .

5053 .

3263 。

3988 。

3855 。

2792 。

3231 。

3855 。

2792 。

3231 。

3855 .

2792 。

3231 。

3302 。

2572。

2878 .

3302 。

2572 。

2878 。

3302 。

2572。

2878 .
7.3871E-03

$6.5892 E-03$

$6.5892 E-03$

$5.5892 E-03$

$6.5892 E-03$

$9.2749 E-03$

$902749 \varepsilon-03$

$9.2749 E-03$

$8.6912 E-03$

$8.6912 E-03$

$8.6912 E-03$

$8.3040 E-03$

$8.3040 E-03$

$8.3040 E-03$ 
N2 - N(CH3)3, 1.011 MICRON D. PARTICLES (CONTINUEDI。

\begin{tabular}{|c|c|c|c|c|c|c|c|}
\hline $\begin{array}{l}\text { RUN } \\
\text { TYPE }\end{array}$ & $\begin{array}{l}\text { YINTI } \\
\text { YINTO } \\
\text { YINTLM }\end{array}$ & $\begin{array}{l}\text { EPEXP } \\
\text { EPCORR } \\
\text { EMT }\end{array}$ & $\begin{array}{l}\text { EPMO } \\
\text { EPSW } \\
\text { EPMA }\end{array}$ & $\begin{array}{l}\text { QINT } \\
\text { PLM } \\
\text { ULM }\end{array}$ & $\begin{array}{l}\text { FILMR } \\
\text { KN } \\
\text { UR }\end{array}$ & $\begin{array}{l}\text { REI } \\
\text { REO } \\
\text { RELM }\end{array}$ & MTC \\
\hline 7065 & $\begin{array}{l}0.8840 \\
0.9400 \\
0.9148\end{array}$ & $\begin{array}{l}0.0792 \\
0.0792 \\
0.5136\end{array}$ & $\begin{array}{l}0.0596 \\
0.0822 \\
0.1113\end{array}$ & $\begin{array}{l}6.0000 E-04 \\
5.5945 E-08 \\
1.294\end{array}$ & $\begin{array}{l}0.0900 \\
0.1107 \\
0.0010\end{array}$ & $\begin{array}{l}2817^{\circ} \\
2390 . \\
2574 .\end{array}$ & $7.3871 E-0.3$ \\
\hline 7065 & $\begin{array}{l}0.8840 \\
0.9400 \\
0.9148\end{array}$ & $\begin{array}{l}0.0832 \\
0.0832 \\
0.5136\end{array}$ & $\begin{array}{l}0.0596 \\
0.0822 \\
0.1113\end{array}$ & $\begin{array}{l}6.0000 E-04 \\
5.5945 E-08 \\
1.294\end{array}$ & $\begin{array}{l}0.0900 \\
0.1107 \\
0.0010\end{array}$ & $\begin{array}{l}2817^{\circ} \\
2390^{\circ} \\
25740\end{array}$ & $7.3 .871 E-03$ \\
\hline 7065 & $\begin{array}{l}0.8840 \\
0.9400 \\
0.9148\end{array}$ & $\begin{array}{l}0.0797 \\
0.0797 \\
0.5136\end{array}$ & $\begin{array}{l}0.0596 \\
0.0822 \\
0.1113\end{array}$ & $\begin{array}{l}6.0000 E-04 \\
5.5945 E-08 \\
1.294\end{array}$ & $\begin{array}{l}0.0900 \\
0.1107 \\
0.0010\end{array}$ & $\begin{array}{l}2817^{\circ} \\
2390^{\circ} \\
2574 .\end{array}$ & $.3871 E-03$ \\
\hline 7066 & $\begin{array}{l}0.9490 \\
0.9730 \\
0.9622\end{array}$ & $\begin{array}{l}0.0330 \\
0.0330 \\
0.4836\end{array}$ & $\begin{array}{l}0.0247 \\
0.0350 \\
0.0492\end{array}$ & $\begin{array}{l}6.0000 E-04 \\
6.0173 E-08 \\
1.231\end{array}$ & $\begin{array}{l}0.1009 \\
0.1190 \\
0.0004\end{array}$ & $\begin{array}{l}2328^{\circ} \\
2170^{\circ} \\
2240^{\circ}\end{array}$ & $6.5892 E-03$ \\
\hline 706 & $\begin{array}{l}0.9490 \\
0.9730 \\
0.9622\end{array}$ & $\begin{array}{l}0.0124 \\
0.0124 \\
0.4836\end{array}$ & $\begin{array}{l}0.0247 \\
0.0350 \\
0.0492\end{array}$ & $\begin{array}{l}6.0000 E-04 \\
6.0173 E-08 \\
1.231\end{array}$ & $\begin{array}{l}0.1009 \\
0.1190 \\
0.0004\end{array}$ & $\begin{array}{l}23280 \\
2170 \\
2240\end{array}$ & $6.5892 E-03$ \\
\hline 7066 & $\begin{array}{l}0.9490 \\
0.9730 \\
0.9622\end{array}$ & $\begin{array}{r}-0.0168 \\
-0.0168 \\
0.4836\end{array}$ & $\begin{array}{l}0.0247 \\
0.0350 \\
0.0492\end{array}$ & $\begin{array}{c}6.0000 E-04 \\
6.0173 E-08 \\
1.231\end{array}$ & $\begin{array}{l}0.1009 \\
0.1190 \\
0.0004\end{array}$ & $\begin{array}{l}23280^{\circ} \\
2170^{\circ} \\
2240^{\circ}\end{array}$ & $6.5892 E-03$ \\
\hline
\end{tabular}


DATA FOR N2 , N(CH3) $3,2.020$ MICRON DIAMETER PARTICLES。

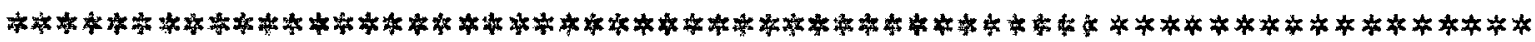

INERT GAS FLOW RATE CONSTANT IN ALL RUNS。

INLET TEMP。 (DEG。 K) 293.0 , PRESSURE (ATM.) 1.000

CALIBRATION CORRECTION: COUNT/TRUE COUNT

INLET: $1.0000+0.0$ *YINTI + 0.0 *YTRNI/YINTI

QUTLET: $0.3500+0.6500 * Y I N T O+0.0$ *YTRNO/YINTO

GAS

$\begin{array}{ll}\text { MOLEC。 WEIGHT } & (\text { G/GMOLE) } \\ \text { DENSITY } & (K G / M * 3) \\ \text { VISCOSIIY } & (K G / M / S E C) \\ \text { MEAN FREE PATH } & \text { (M) } \\ \text { VOLUME } \$(1 / 3) & (M / G M O L E * *(1 / 3))\end{array}$

DIFFUSIVITY

$(M+2 / S E C)$

RUN YINTI

TYPE YINTO

YINTLM

367680.6890

0.8340

0.7667

367680.6890

0.8340

0.7667

367680.6890

0.8340

0.7667

367680.6890

0.8340

0.7667

367690.7780

0. 8870

0.8374

367690.7780

0.8870

0.8374

367690.7780

0. 8870

0.8374

367690.7780

0.8870

0.8374

367690.7780

0.8870

0.8374
EPEXP EPMD

EPCORR EPSW

EMT

0.2582

0.1685

0.5590

0.2770

0.1896

0.5590

0.3094

0.2259

0.5590

0.3002

0.2156

0.5590

0.2061

0.1432

0.5535

0.2761

0.2187

0.5535

0.2220

0.1603

0.5535

0.2141

0.1518

0.5535

0.2109

0.1483

0.5535
EPMA

QINT

PLM

ULM

N2

(INERT)

$$
\begin{aligned}
& 28.02 \\
& 1.165
\end{aligned}
$$

1. $7.480 E-05$

6.3900E-08

3. $1190 E-02$
$\mathrm{N}(\mathrm{CH} 3) 3$

(TRANSFERRED)

$$
59.11
$$$$
2.510
$$

7. $5300 E-06$

$1.8800 E-08$

4. 5360E-02

\section{$8.4411 E-06$}

$$
0.17396 .0000 E-04 \quad 0.0717
$$

$0.2214405287 \mathrm{E}-080.0448$

$0.2727 \quad 1.544$

0.0027

0.1739 6.00OOE-04 0.0717

$0.2214 \quad 405287 E-08$ 0.0448

$0.2727 \quad 1.544$

0.0027

$0.17396 .0000 E-04 \quad 0.0717$

$0.2214405287 E-08$

0.0448

$0.2727 \quad 1.544$

0.0027

$0.1739 \quad 6.0000 E-04 \quad 0.0717$

$0.2214 \quad 4.5287 E-08$

0.0448

0.0027

$0.2727 \quad 1.544$

0.0765

$0.16224 .9955 E-08 \quad 0.0495$

0.20801 .414

0.0019

$0.1229600000 E-040.0765$

$0.1622 \quad 4.9955 E-080.0495$

$0.2080 \quad 1.414$

0.0019

D.1229 6.0000E-04 0.0765

$0.1622 \quad 409955 E-08 \quad 0.0495$

0.20801 .414

0.0019

$0.12296 .0000 E-040.0765$

$0.1622 \quad 409955 E-08 \quad 0.0495$

$\begin{array}{lll}0.2080 & 1.414 & 0.0019\end{array}$

$0.12296 .0000 E-040.0765$

$0.1622 \quad 409955 E-080.0495$

$0.2080 \quad 1.414$

0.0019
5053.9.2749E-03

3263 。

3988 .

5053。9.2749E-03

3263 。

3988 .

5053.9.2749E-03

3263.

3988.

5053.9.2749E-03

3263.

3988 。

3855 。

2792 .

3231 。

3855 .

2792 .

3231 。

3855 。

2792 。

3231 。

3855 .

2792 。

3231 。

3855 .

2792 。

3231 。
$8.6912 E-03$

8. $6912 E-03$

8. $6912 E-03$

$8.6912 E-03$

$8.6912 E-03$ 
N2 - N(CH3)3,

2.020 MICRON

PART ICLES

(CONT INUED).

\begin{tabular}{|c|c|c|c|c|c|c|c|}
\hline $\begin{array}{l}\text { RUN } \\
\text { TYPE }\end{array}$ & $\begin{array}{l}\text { YINTI } \\
\text { YINTD } \\
\text { YINTLM }\end{array}$ & $\begin{array}{l}\text { EPEXP } \\
\text { EPCORR } \\
\text { EMT }\end{array}$ & $\begin{array}{l}\text { EPMO } \\
\text { EPSW } \\
\text { EPMA }\end{array}$ & $\begin{array}{l}\text { QINT } \\
\text { PLM } \\
\text { ULM }\end{array}$ & $\begin{array}{l}\text { FILMR } \\
\text { KN } \\
\text { UR }\end{array}$ & $\begin{array}{l}\text { REI } \\
\text { REO } \\
\text { RELM }\end{array}$ & MTC \\
\hline 6771 & $\begin{array}{l}0.8840 \\
0.9400 \\
0.9148\end{array}$ & $\begin{array}{l}0.1189 \\
0.0831 \\
0.5136\end{array}$ & $\begin{array}{l}0.0596 \\
0.0822 \\
0.1113\end{array}$ & $\begin{array}{l}6.0000 E-04 \\
5.5945 E-08 \\
1.294\end{array}$ & $\begin{array}{l}0.0900 \\
0.0554 \\
0.0010\end{array}$ & $\begin{array}{l}2817^{\circ} \\
2390^{\circ} \\
2574^{\circ}\end{array}$ & $7.3871 E-03$ \\
\hline 36771 & $\begin{array}{l}0.8840 \\
0.9400 \\
0.9148\end{array}$ & $\begin{array}{l}0.1877 \\
0.1547 \\
0.5136\end{array}$ & $\begin{array}{l}0.05 .96 \\
0.0822 \\
0.1113\end{array}$ & $\begin{array}{l}6.0000 E-04 \\
5.5945 E-08 \\
1.294\end{array}$ & $\begin{array}{l}0.0900 \\
0.0554 \\
0.0010\end{array}$ & $\begin{array}{l}2817^{\circ} \\
2390^{\circ} \\
2574^{\circ}\end{array}$ & $7.3871 E-03$ \\
\hline 6771 & $\begin{array}{l}0 . \\
0.5 \\
0.5\end{array}$ & $\begin{array}{l}0 . \\
0 . \\
0 .\end{array}$ & $\begin{array}{l}0.0596 \\
0.0822 \\
0.1113\end{array}$ & $\begin{array}{l}6.0000 E-04 \\
5.5945 E-08 \\
1.294\end{array}$ & $\begin{array}{l}0.0900 \\
0.0554 \\
0.0010\end{array}$ & $\begin{array}{l}2817^{\circ} \\
2390^{\circ} \\
2574^{\circ}\end{array}$ & $7.3871 E-03$ \\
\hline 72 & $\begin{array}{l}0.9490 \\
0.9730 \\
0.9622\end{array}$ & $\begin{array}{l}0.0734 \\
0.0568 \\
0.4836\end{array}$ & $\begin{array}{l}0.0247 \\
0.0350 \\
0.0492\end{array}$ & $\begin{array}{c}6.0000 E-04 \\
6.0 .173 E-08 \\
1.231\end{array}$ & $\begin{array}{l}0.1009 \\
0.0596 \\
0.0004\end{array}$ & $\begin{array}{l}2328 . \\
2170^{\circ} \\
2240^{\circ}\end{array}$ & $6.5892 E-03$ \\
\hline 6772 & $\begin{array}{l}0.9490 \\
0.9730 \\
0.9622\end{array}$ & $\begin{array}{l}0.0973 \\
0.0812 \\
0.4836\end{array}$ & $\begin{array}{l}0.0247 \\
0.0350 \\
0.0492\end{array}$ & $\begin{array}{l}6.0000 E-04 \\
6.0173 E-08 \\
1.231\end{array}$ & $\begin{array}{l}0.1009 \\
0.0596 \\
0.0004\end{array}$ & $\begin{array}{l}2328 \circ \\
2170^{\circ} \\
2240^{\circ}\end{array}$ & $.5892 z-03$ \\
\hline 76 & $\begin{array}{l}0.6890 \\
0.8340 \\
0.7667\end{array}$ & $\begin{array}{l}0.3277 \\
0.2464 \\
0.5590\end{array}$ & $\begin{array}{l}0.1739 \\
0.2214 \\
0.2727\end{array}$ & $\begin{array}{l}6.0000 E-04 \\
4.5287 E-08 \\
1.544\end{array}$ & & & 13 \\
\hline & $\begin{array}{l}0.6 \\
0.8 \\
0 .\end{array}$ & & $\begin{array}{l}0.1739 \\
0.2214 \\
0.2727\end{array}$ & $\begin{array}{l}6.0000 E-04 \\
4.5287 E-08\end{array}$ & & & 9.27 \\
\hline 76 & $\begin{array}{l}0.6 \\
0.8 \\
0.7\end{array}$ & & $\begin{array}{l}0.1739 \\
0.2214 \\
0.2727\end{array}$ & $\begin{array}{l}6.0000 E-04 \\
4.5287 E-08 \\
1.544\end{array}$ & $\begin{array}{l}0.0717 \\
0.0448 \\
0.0027\end{array}$ & $\begin{array}{l}5053 \circ \\
3263 . \\
3988 .\end{array}$ & $9.2749 E-03$ \\
\hline & $\begin{array}{l}0.6 \\
0.8 \\
0.7\end{array}$ & $\begin{array}{l}097 \\
262 \\
590\end{array}$ & $\begin{array}{l}0.1739 \\
0.2214 \\
0.2727\end{array}$ & $\begin{array}{l}6.0000 E-04 \\
4.5287 E-08 \\
1.544\end{array}$ & $\begin{array}{l}0.0717 \\
0.0448 \\
0.0027\end{array}$ & $\begin{array}{l}5053^{\circ} \\
3263^{\circ} \\
3988^{\circ}\end{array}$ & 9.2 \\
\hline & $\begin{array}{l}0.8300 \\
0.9150 \\
0.8767\end{array}$ & $\begin{array}{l}844 \\
367 \\
465\end{array}$ & $\begin{array}{l}0.0929 \\
0.1253 \\
0.1649\end{array}$ & $\begin{array}{l}6.0000 E-04 \\
5.2869 E-08 \\
1.351\end{array}$ & $\begin{array}{l}0.0800 \\
0.0523 \\
0.0015\end{array}$ & $\begin{array}{l}3302^{\circ} \\
2572^{\circ} \\
2878\end{array}$ & 80 \\
\hline & $\begin{array}{l}0.8300 \\
0.9150 \\
0.8767\end{array}$ & $\begin{array}{l}035 \\
569 \\
465\end{array}$ & $\begin{array}{l}929 \\
253 \\
649\end{array}$ & $\begin{array}{l}6.0000 E-04 \\
5.2869 E-08 \\
1.351\end{array}$ & $\begin{array}{l}20 \\
23 \\
5\end{array}$ & $\begin{array}{l}3302^{\circ} \\
2572^{\circ} \\
2870^{\circ}\end{array}$ & -03 \\
\hline $780-8$ & $\begin{array}{l}0.8300 \\
0.9150 \\
0.8767\end{array}$ & $\begin{array}{l}208 \\
752 \\
465\end{array}$ & $\begin{array}{l}929 \\
253 \\
649\end{array}$ & $\begin{array}{l}6.0000 E-04 \\
5.2869 E-08 \\
1.351\end{array}$ & $\begin{array}{l}0.1 \\
0.0 \\
0.0\end{array}$ & $\begin{array}{l}2 . \\
2 . \\
8 .\end{array}$ & -03 \\
\hline 78 & $\begin{array}{l}0.8840 \\
0.9400 \\
0.9148\end{array}$ & $\begin{array}{l}137 \\
777 \\
136\end{array}$ & $\begin{array}{l}0.0596 \\
0.0822 \\
0.1113\end{array}$ & $\begin{array}{l}6.0000 E-04 \\
5.5945 E-08 \\
1.294\end{array}$ & $\begin{array}{l}50 \\
54 \\
0\end{array}$ & $\begin{array}{l}2817^{\circ} \\
2390 。 \\
2574\end{array}$ & -03 \\
\hline & $\begin{array}{l}0.8 \\
0.5 \\
0.5 \\
0.8\end{array}$ & $\begin{array}{l}0.1372 \\
0.1022 \\
0.5136 \\
0.1465\end{array}$ & $\begin{array}{l}0.0596 \\
0.0822 \\
0.1113 \\
0.0596\end{array}$ & $\begin{array}{l}6.0000 E-04 \\
5.5945 E-08 \\
1.294\end{array}$ & $\begin{array}{l}0.0900 \\
0.0554 \\
0.0010 \\
0.0900\end{array}$ & $\begin{array}{l}2817 \\
2390^{\circ} \\
2574 \circ \\
2817\end{array}$ & -03 \\
\hline . & $\begin{array}{l}0.8840 \\
0.9400 \\
0.9148\end{array}$ & $\begin{array}{l}0.1465 \\
0.1119 \\
0.5136\end{array}$ & $\begin{array}{l}0.0596 \\
0.0822 \\
0.1113\end{array}$ & $\begin{array}{l}6.0000 E-04 \\
5.5945 E-08 \\
1.294\end{array}$ & $\begin{array}{l}0.0900 \\
0.0554 \\
0.0010\end{array}$ & $\begin{array}{l}2817^{\circ} \\
2390^{\circ} \\
2574^{\circ}\end{array}$ & 70 \\
\hline & $\begin{array}{l}0.9490 \\
0.9730 \\
0.9622\end{array}$ & $\begin{array}{l}0.1163 \\
0.1005 \\
0.4836\end{array}$ & $\begin{array}{l}0.0247 \\
0.0350 \\
0.0492\end{array}$ & $\begin{array}{l}6.0000 E-04 \\
6.0173 E-08 \\
1.231\end{array}$ & $\begin{array}{l}0.1009 \\
0.0596 \\
0.0004\end{array}$ & $\begin{array}{l}2328^{\circ} \\
2170^{\circ} \\
2240^{\circ}\end{array}$ & U. \\
\hline
\end{tabular}


(CONTINUED)。

\begin{tabular}{|c|c|c|c|c|c|c|c|}
\hline $\begin{array}{l}\text { RUN } \\
\text { TYPE }\end{array}$ & $\begin{array}{l}\text { YINTI } \\
\text { YINTO } \\
\text { YINTLM }\end{array}$ & $\begin{array}{l}\text { EPEXP } \\
\text { EPCORR } \\
\text { EMT }\end{array}$ & $\begin{array}{l}\text { EPMO } \\
\text { EPSW } \\
\text { EPMA }\end{array}$ & $\begin{array}{l}\text { QINT } \\
\text { PLM } \\
\text { ULM }\end{array}$ & $\begin{array}{l}\text { FILMR } \\
K N \\
U R\end{array}$ & $\begin{array}{l}\text { REI } \\
\text { RED } \\
\text { RELM }\end{array}$ & MTC \\
\hline 36779 & $\begin{array}{l}0.9490 \\
0.9730 \\
0.9622\end{array}$ & $\begin{array}{l}0.0894 \\
0.0731 \\
0.4836\end{array}$ & $\begin{array}{l}0.0247 \\
0.0350 \\
0.0497\end{array}$ & $\begin{array}{l}6.0000 E-04 \\
6.0173 E-08 \\
1.231\end{array}$ & $\begin{array}{l}0.1009 \\
0.0596 \\
0.0004\end{array}$ & $\begin{array}{l}2328^{\circ} \\
2170 \% \\
2240^{\circ}\end{array}$ & $6.5892 E-03$ \\
\hline 79 & $\begin{array}{l}0.9490 \\
0.9730 \\
0.9622\end{array}$ & $\begin{array}{l}0.04030 \\
0.0464 \\
0.0294 \\
0.4836\end{array}$ & $\begin{array}{l}0.0472 \\
0.0247 \\
0.0350 \\
0.0492\end{array}$ & $\begin{array}{l}6.0000 E-04 \\
6.0173 E-08 \\
1.231\end{array}$ & $\begin{array}{l}0.0004 \\
0.1009 \\
0.0596 \\
0.0004\end{array}$ & $\begin{array}{l}2 \angle 400 \\
23280 \\
2170^{\circ} \\
2240^{\circ}\end{array}$ & $6.5892 E-03$ \\
\hline 6797 & $\begin{array}{l}0.6890 \\
0.8340 \\
0.7667\end{array}$ & $\begin{array}{l}0.2798 \\
0.1927 \\
0.5590\end{array}$ & $\begin{array}{l}0.1739 \\
0.2214 \\
0.2727\end{array}$ & $\begin{array}{c}6.0000 E-04 \\
4.5287 E-08 \\
1.544\end{array}$ & $\begin{array}{l}0.0 \\
0.0 \\
0.0\end{array}$ & $\begin{array}{l}5053 \\
3263 . \\
3988\end{array}$ & $9.2749 E-03$ \\
\hline 6797 & $\begin{array}{l}0.6 \\
0.8 \\
0.7\end{array}$ & $\begin{array}{l}029 \\
186 \\
590\end{array}$ & $\begin{array}{l}0.1739 \\
0.2214 \\
0.2727\end{array}$ & $\begin{array}{c}6.0000 E-04 \\
4.5287 E-08 \\
1.544\end{array}$ & $\begin{array}{l}0 . \\
0 . \\
0 .\end{array}$ & $\begin{array}{l}5053^{\circ} \\
3263^{\circ} \\
3988 .\end{array}$ & 9.27 \\
\hline 6797 & $\begin{array}{l}0.6 \\
0.8 \\
0.7\end{array}$ & & $\begin{array}{l}0.1739 \\
0.2214 \\
0.2727\end{array}$ & $\begin{array}{c}6.0000 E-04 \\
4.5287 E-08 \\
1.54 .4\end{array}$ & $\begin{array}{l}0.0717 \\
0.0448 \\
0.0027\end{array}$ & $\begin{array}{l}5053 . \\
3263 . \\
3988\end{array}$ & -03 \\
\hline 16798 & $\begin{array}{l}0.7780 \\
0.8870 \\
0.8374\end{array}$ & $\begin{array}{l}0.2587 \\
0.1999 \\
0.5535\end{array}$ & $\begin{array}{l}0.1229 \\
0.1622 \\
0.2080\end{array}$ & $\begin{array}{l}6.0000 E=04 \\
4.9955 E=08 \\
1.414\end{array}$ & $\begin{array}{l}0.0765 \\
0.0495 \\
0.0019\end{array}$ & $\begin{array}{l}3855^{\circ} \\
2792^{\circ} \\
3231^{\circ}\end{array}$ & -03 \\
\hline 98 & $\begin{array}{l}0.7780 \\
0.8870 \\
0.8374\end{array}$ & $\begin{array}{l}0.2618 \\
0.2033 \\
0.5535\end{array}$ & $\begin{array}{l}0.1229 \\
0.1622 \\
0.2080\end{array}$ & $\begin{array}{l}6.0000 E-04 \\
4.9955 E-08 \\
1.414\end{array}$ & $\begin{array}{l}0.0765 \\
0.0495 \\
0.0019\end{array}$ & $\begin{array}{l}3855^{\circ} \\
2792^{\circ} \\
3231^{\circ}\end{array}$ & 8. \\
\hline 67 & $\begin{array}{l}0.7780 \\
0.8870 \\
0.8374\end{array}$ & $\begin{array}{l}0.2501 \\
0.1907 \\
0.5535\end{array}$ & $\begin{array}{l}0.1229 \\
0.1622 \\
0.2080\end{array}$ & $\begin{array}{l}6.0000 E-04 \\
4.9955 E-08 \\
1.414\end{array}$ & $\begin{array}{l}0.1 \\
0.0 \\
0 .\end{array}$ & $\begin{array}{l}50 \\
2 . \\
1 .\end{array}$ & 8.69 \\
\hline 6 & $\begin{array}{l}0_{0} \\
0_{0}\end{array}$ & & $\begin{array}{l}0 . \\
0 . \\
0 .\end{array}$ & $\begin{array}{l}O E-04 \\
9 E-08 \\
1\end{array}$ & $\begin{array}{l}0.0 \\
0.0 \\
0.0\end{array}$ & $\begin{array}{l}3302^{\circ} \\
2572^{\circ} \\
2878 .\end{array}$ & -03 \\
\hline 6799 & $\begin{array}{l}0 . \\
0 . \\
0 .\end{array}$ & $\begin{array}{l}0 . \\
0 . \\
0 .\end{array}$ & $\begin{array}{l}29 \\
53 \\
49\end{array}$ & $\begin{array}{l}6.0000 E-04 \\
5.2869 E-08 \\
1.351\end{array}$ & $\begin{array}{l}0.0 \\
0.0 \\
0.0\end{array}$ & $\begin{array}{l}3302^{\circ} \\
2572^{\circ} \\
2878^{\circ}\end{array}$ & -03 \\
\hline 66799 & $\begin{array}{l}0.8300 \\
0.9150 \\
0.8767\end{array}$ & $\begin{array}{l}0.1745 \\
0.1262 \\
0.5465\end{array}$ & $\begin{array}{l}0.0929 \\
0.1253 \\
0.1649\end{array}$ & $\begin{array}{l}6.0000 E-04 \\
5.2869 E-08 \\
1.351\end{array}$ & $\begin{array}{l}0.08 \\
0.05 \\
0.00\end{array}$ & $\begin{array}{l}3302^{\circ} \\
2572^{\circ} \\
2878 \circ\end{array}$ & 03 \\
\hline 36800 & $\begin{array}{l}0.8840 \\
0.9400 \\
0.9148\end{array}$ & $\begin{array}{l}0.1602 \\
0.1261 \\
0.5136\end{array}$ & $\begin{array}{l}0.0596 \\
0.0822 \\
0.1113\end{array}$ & $\begin{array}{l}6.0000 E-04 \\
5.5945 E-08 \\
1.294\end{array}$ & $\begin{array}{l}0.09 \\
0.05 \\
0.00\end{array}$ & $\begin{array}{l}2817^{\circ} \\
2390^{\circ} \\
2574 \circ\end{array}$ & 7. \\
\hline 68 & $\begin{array}{l}0.8840 \\
0.9400 \\
0.9148\end{array}$ & $\begin{array}{l}0.1588 \\
0.1247 \\
0.5136\end{array}$ & $\begin{array}{l}0.0596 \\
0.0822 \\
0.1113\end{array}$ & $\begin{array}{l}6.0000 E-04 \\
5.5945 E-08 \\
1.294\end{array}$ & $\begin{array}{l}0 . \\
0 . \\
0 .\end{array}$ & $\begin{array}{l}2817^{\circ} \\
2390^{\circ} \\
2574 \circ\end{array}$ & $7.3871 E-03$ \\
\hline & $\begin{array}{l}0.8840 \\
0.9400 \\
0.9148\end{array}$ & $\begin{array}{l}0.1708 \\
0.1371 \\
0.5136\end{array}$ & $\begin{array}{l}0.0596 \\
0.0822 \\
0.1113\end{array}$ & $\begin{array}{l}6.0000 E-04 \\
5.5945 E-08 \\
1.294\end{array}$ & $\begin{array}{l}0.0 \\
0.0 \\
0.0\end{array}$ & $\begin{array}{l}2817^{\circ} \\
2390 \\
2574^{\circ}\end{array}$ & $7.3871 E-03$ \\
\hline & $\begin{array}{l}0.9490 \\
0.9730 \\
0.9622\end{array}$ & $\begin{array}{l}0.1151 \\
0.0993 \\
0.4836 \\
0.0680\end{array}$ & $\begin{array}{l}0.0247 \\
0.0350 \\
0.0492 \\
0.0247\end{array}$ & $\begin{array}{l}6.0000 E-04 \\
6.0173 E-08 \\
1.231 \\
6.0000 E-04\end{array}$ & $\begin{array}{l}0.1009 \\
0.0596 \\
0.0004\end{array}$ & $\begin{array}{l}2328^{\circ} \\
2170^{\circ} \\
2240^{\circ}\end{array}$ & 6.58921 \\
\hline 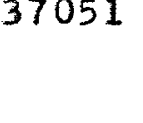 & $\begin{array}{l}0.9490 \\
0.9730 \\
0.9622\end{array}$ & $\begin{array}{l}0.0680 \\
0.0514 \\
0.4836\end{array}$ & $\begin{array}{l}0.0247 \\
0.0350 \\
0.0492\end{array}$ & $\begin{array}{l}6.0000 E-04 \\
6.0173 E-08 \\
1.231 .\end{array}$ & $\begin{array}{l}0.1009 \\
0.0596 \\
0.0004\end{array}$ & $\begin{array}{l}23280 \\
2170^{\circ} \\
2240 .\end{array}$ & 605 \\
\hline
\end{tabular}


N2 - N(CH3)3, 2.020 MICRON D. PARTICLES (IONTINUED)。

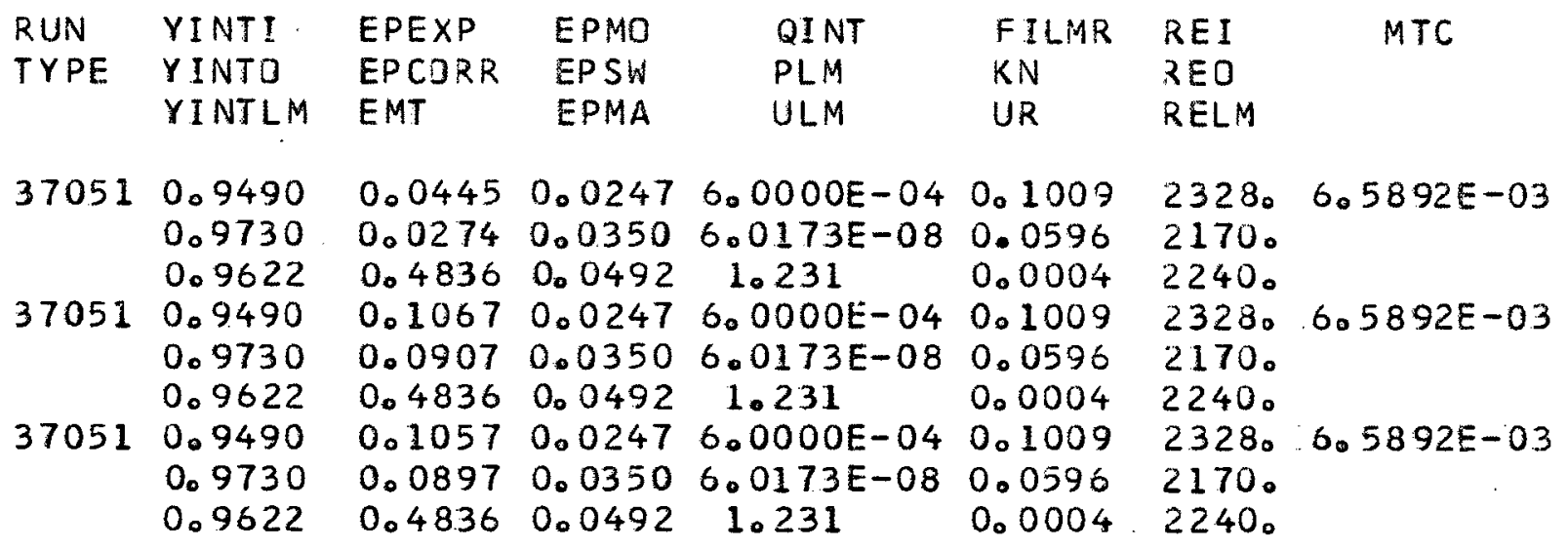


TABLE XXVIII

DATA FOR N2 - N(CH3)3,5.700 MICRON DIAMETER PARTICLES。

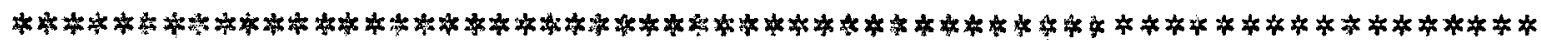

INERT GAS FLOW RATE CONSTANT IN ALL RUNS。

INLET TEMP。 (DEG。K) 293.0 , PRESSURE (ATM。) 1.000

CALIBRATION CORRECTION: COUNT/TRUE COUNT

INLET: $1.0000+0.0$ YINTI + +0.0 *YTRNI/YIVTI DUTLET: $1.0000+0.0$ *YINTO

GAS

$$
\begin{aligned}
& \text { N2 } \\
& \text { (INERT) }
\end{aligned}
$$

$\begin{array}{ll}\text { MOLEC。 WEIGHT } & (\text { G/GMOLE) } \\ \text { DENSITY } & (K G / M * 3) \\ \text { VISCOSITY } & (K G / M / S E C) \\ \text { MEAN FREE PATH } & (M) \\ \text { VOLUME* } *(1 / 3) & (M / G M O L E *(1 / 3))\end{array}$

DIFFUSI VITY

$(M * 2 / S E C)$

$$
\begin{aligned}
& 28.02 \\
& 1.165
\end{aligned}
$$

1. $7480 E-05$

6. $3900 E-08$

3. $1190 E-02$
$\mathrm{N}(\mathrm{CH} 3) 3$

(TRANSFERRED)

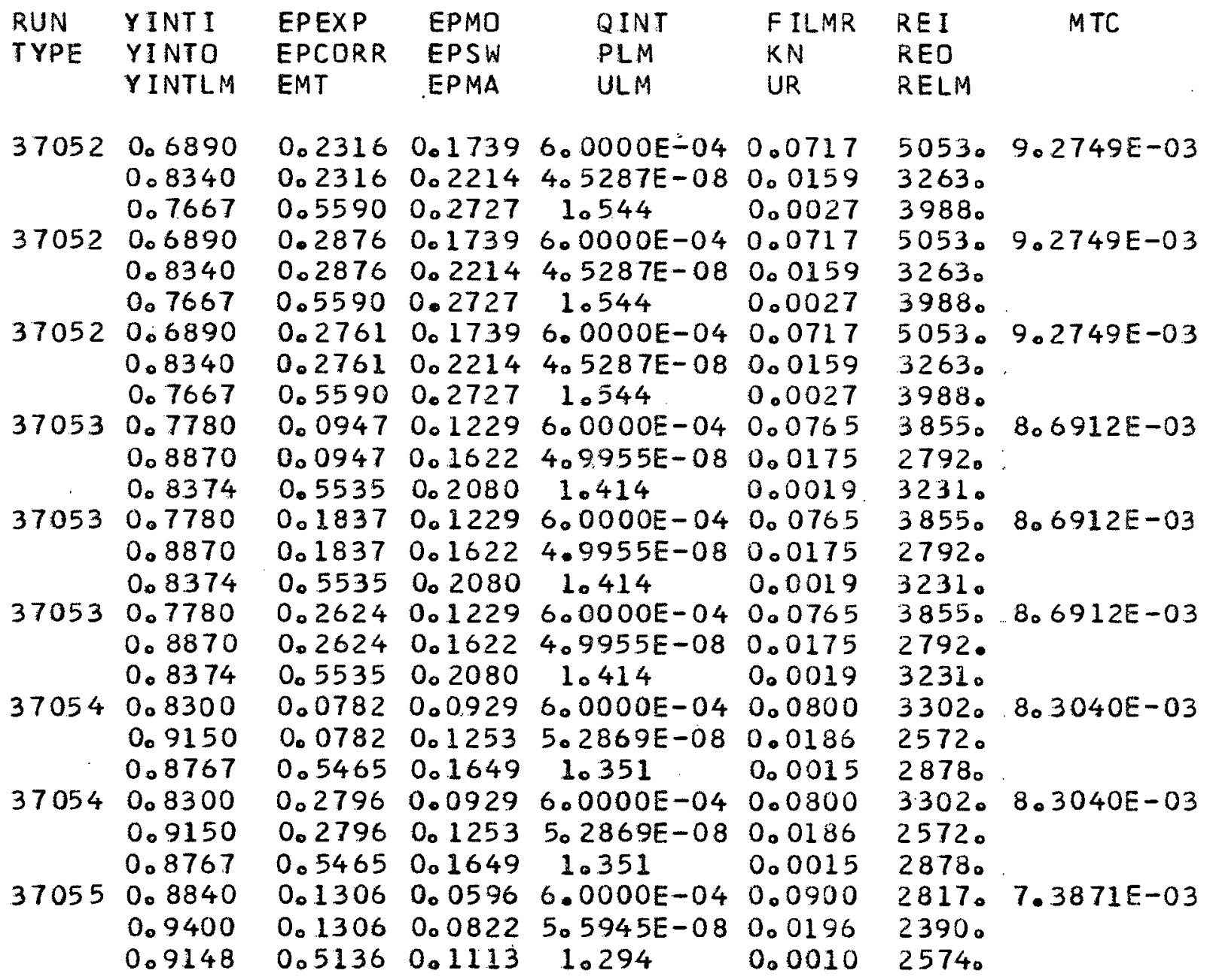

$$
\begin{aligned}
& 59.11 \\
& 2.510
\end{aligned}
$$

7. 5300 E-06

1. 8800 E-08

4. $5360 E-02$

\section{$8.4411 E-06$}


Appendix E

\section{STATISTICAL ANALYSIS}

\section{E.1 General Position of Data}

For every set of data, the mean offset of the experimental results from the predictions of each of the three theoretical models was calculated. The mean offset is the average of all the differences between the experimental and theoretical fractional efficiencies. The standard deviation of these differences was also calculated, and was used to determine the significance of the offset at the $90 \%$ and $95 \%$ confidence levels using the statistical t-test. The calculation was done by computer using the program UBC SFRP (Le, (1975)).

\section{E.2 Transition Regime Behaviour and Inertial Deposition}

No simple test to detect transition regime behaviour could be devised. A transition regime model for particle removal was therefore created, and used in the tests.

In order to construct the model it was postulated that if particle behaviour fell into the transition regime, then the 
particle velocity would be given by

$$
v_{p}=f v_{S W}+(1-f) v_{M A},
$$

where $\mathrm{v}_{\mathrm{MA}}$ and $\mathrm{v}_{\mathrm{SW}}$ are the mean mass velocity and schmitt and Waldmann's velocity respectively, and $f$ is a parameter of order 1 or smaller. It is straightforward to derive the corresponding expression for particle removal efficiency, using the film theory approach (see Whitmore and Meisen, (1973)), to yield

$$
\varepsilon_{T R}=1-\left[\left(1-\varepsilon_{S W}\right)^{f}\left(1-\varepsilon_{M A}\right)^{1-f}\right],
$$

where $\varepsilon_{\mathrm{TR}}$ is the transition regime efficiency. (The corresponding derivation for the general theory is not obvious, and may not be possible.) By means of the binomial expression, it can be shown that this expression can be approximated by

$$
\varepsilon_{\mathrm{TR}}=f \varepsilon_{\mathrm{SW}}+(I-f) \varepsilon_{\mathrm{MA}},
$$

where $\varepsilon_{M A}$ and $\varepsilon_{S W}$ are small $\left(\varepsilon_{M A}, \varepsilon_{S W}<<1\right)$. It was found that this linearised result agreed with the more accurate expression to within 0.01 fractional efficiency units for the values of $\varepsilon_{M A}$ and $\varepsilon_{S W}$ corresponding to experimental conditions in this work. The simple equation was therefore satisfactory for use in the analysis.

If the particles are in the transition regime, then the parameter $f$ will be a function of Knudsen number. Over a 
small range of knudsen number values, the relationship can be regarded as linear, so that

$$
\mathrm{f}=\mathrm{A}_{r}+\mathrm{B}_{r} \mathrm{Kn},
$$

where $A_{r}$ and $B_{r}$ are constants. It follows that

$$
\varepsilon_{\mathrm{TR}}=\mathrm{A}_{\mathrm{r}}\left(\varepsilon_{\mathrm{SW}}-\varepsilon_{\mathrm{MA}}\right)+\mathrm{B}_{\mathrm{r}}\left(\varepsilon_{\mathrm{SW}}-\varepsilon_{\mathrm{MA}}\right) \mathrm{Kn}+\varepsilon_{\mathrm{MA}} .
$$

If inertial deposition is also important, the efficiency will depend on the stopping distance parameter, S (see Chapter 6). The dependence can be assumed to be linear over the range of this parameter encountered in the experimental work. Hence, the final expression for particle removal efficiency becomes:

$$
\varepsilon_{p}=A_{r}\left(\varepsilon_{S W}-\varepsilon_{M A}\right)+B_{r}\left(\varepsilon_{S W}-\varepsilon_{M A}\right) K n+C_{r} S+\varepsilon_{M A} .
$$

The tests for both transition regime behaviour and inertial deposition involved attempting to correlate the experimental values for $\varepsilon_{p}$ with the corresponding values of $\left(\varepsilon_{S W}-\varepsilon_{M A}\right)$, $\mathrm{Kn}$, and $\mathrm{S}$, using the previous equation. The values of the latter three variables were based on the measured experimental conditions (see Appendix $\mathrm{C}$ ). The tests were made on each individual data set, and on each set of grouped data (see Chapter 6).

The correlation was sought using a stepwise regression analysis. In the analysis, variables are discarded as not significant if the variance attributable to them is not significantly 
different from the residual variance attributable to random scatter in the data. The significance level is determined by means of the F-test. The analysis was made using significance levels of $90 \%$ (significant) and 95\% (highly significant). The calculation was done by computer using the program previously mentioned.

The results of these tests did not confirm the hypothesis that the data indicated transition regime behaviour. Where the coefficients $A_{r}$ and $B_{r}$ were found to be significant, it was clear from their values that the correlation was due not to Knudsen number, but to other causes, probably systematic errors in the data. Large values of these coefficients imply that transition behaviour is confined to a very small knudsen number range, which is not realistic.

Particularly for the ungrouped data, there was a strong inter-correlation between the two variables $\left(\varepsilon_{S W}-\varepsilon_{M A}\right)$ and $\left(\varepsilon_{S W}-\varepsilon_{M A}\right) K n$. This arose because the variation of the Knudsen number within one set of data was normally small. Where the supposedly independent variables in a regression analysis are actually interdependent, as in this case, the regression method may have difficulty in correctly attributing any correlation to the appropriate variable. The values obtained for $A_{r}$ and $B_{r}$ might therefore reflect this problem rather than systematic errors. When the knudsen number range is small, the influence of variation in Knudsen number on the removal efficiency should be negligible. This variation can be neglected by discarding the term ${ }^{B}{ }_{r}\left(\varepsilon_{S W}-\varepsilon_{M A}\right) K n$ from the analysis. If the correlation with 
$\left(\varepsilon_{S W}-\varepsilon_{M A}\right)$ were still found to be significant, this would substantiate the hypothesis of transition behaviour, and indicate that the previous unrealistic values of $A_{r}$ and $B_{r}$ were due to intercorrelation of variables.

When these tests were made, the correlation with $\left(\varepsilon_{S W}-\varepsilon_{M A}\right)$ was found to be generally not significant. It was therefore concluded that transition regime behaviour could not be statistically demonstrated, probably because of the presence of systematic errors in the data. This does not mean, however, that transition behaviour was not in effect, since the possible systematic errors, as listed in Table IV, are much larger than is necessary to confound the statistical analysis. Also, it is possible, though not probable, that the transition regime model was not accurate enough for use in these tests.

For some sets of data, the correlation with the stopping distance parameter was significant, typically indicating an inertial loss for $1 \mu \mathrm{m}$ particles in the order of $0.5 \%$ at a Reynolds number of 1000 . This was higher than expected, but not obviously unrealistic. At the same time, the correlation with stopping distance was negative in a few cases, which was clearly impossible. It is therefore concluded that systematic errors were interfering with this test, but that inertial losses must in any case have been very small. 